\title{
Convergent Total Synthesis of Hikizimycin Enabled by Intermolecular Radical Addition to Aldehyde
}

Haruka Fujino, Takumi Fukuda, Masanori Nagatomo and Masayuki Inoue*

Graduate School of Pharmaceutical Sciences

The University of Tokyo, Hongo, Bunkyo-ku, Tokyo 113-0033, Japan

Fax: $(+81) 3-5841-0568$

E-mail: inoue@mol.f.u-tokyo.ac.jp

Supporting Information

79 pages

Contents:

page

$\begin{array}{ll}\text { 1. Experimental procedures } & \mathrm{S} 2\end{array}$

2. Comparison of ${ }^{1} \mathrm{H}$ and ${ }^{13} \mathrm{C}$ NMR data of the reported and synthetic $\quad$ S26 hikizimycin (1) in $\mathrm{D}_{2} \mathrm{O}$

3. Investigation of the effect of $\mathrm{C} 4-\mathrm{N}$-protective group and $\quad \mathrm{S} 30$

$\mathrm{C} 1$-stereochemistry on the radical addition reactions

4. X-ray structures and crystallographic data of 28- $\alpha \quad$ S32

5. Structural determination of 29- $\alpha \quad$ S34

6. Structural determination of $\mathbf{3 0 - \alpha} \boldsymbol{S} 34$

$\begin{array}{ll}\text { 7. Computational modeling of } \mathbf{B} & \text { S37 }\end{array}$

$\begin{array}{ll}\text { 8. CP3 analysis of } \mathbf{6}-\boldsymbol{\alpha} \text { and } \mathbf{6}-\boldsymbol{\beta} & \text { S39 }\end{array}$

9. References S41

10. NMR charts $\quad$ S43

$\begin{array}{ll}\text { 11. HPLC charts for purification of hikizimycin (1) } & \text { S79 }\end{array}$ 


\section{Experimental procedures}

General method: $\quad \mathrm{Et}_{3} \mathrm{~B}-\mathrm{mediated}$ radical reactions were carried out under air unless otherwise noted. All other reactions sensitive to air or moisture were carried out under argon atmosphere in dry solvents, unless otherwise noted. $\mathrm{CH}_{2} \mathrm{Cl}_{2}$, DMF and THF were purified by Glass Contour solvent dispensing system (Nikko Hansen \& Co., Ltd.). All other reagents were used as supplied. Analytical thin-layer chromatography (TLC) was performed using E. Merck Silica gel $60 \mathrm{~F}_{254}$ pre-coated plates $(0.25 \mathrm{~mm})$. Preparative thin layer chromatography (PTLC) was performed using Merck silica gel $60 \mathrm{~F}_{254}$ pre-coated plates $(0.5 \mathrm{~mm})$. Flash column chromatography was performed using 40-50 $\mu \mathrm{m}$ Silica Gel 60N (Kanto Chemical Co., Inc.), 32-53 $\mu \mathrm{m}$ Silica-gel BW-300 (Fuji Silysia Chemical Ltd.), or $60 \mu \mathrm{m}$ amine-modified Silica Gel Chromatorex NH-DM2035 (Fuji Silysia Chemical Ltd.)]. Automated flash chromatography was conducted with a Yamazen Smart Flash EPCLC AI-580S system using prefilled silica gel columns. Unless otherwise noted, Silica Gel $60 \mathrm{~N}$ was used for the purification. Preparative gel permeation chromatography (GPC) was performed on LaboACE LC-5060 (Japan Analytical Industry Co., Ltd.) using UV (254 nm) and Refractive Index (RI) detectors in $\mathrm{CHCl}_{3}$ with flow rate $10.0 \mathrm{~mL} / \mathrm{min}$, equipped with JAIGEL-2HR column. High performance liquid chromatography (HPLC) were equipped with a JASCO HPLC system (pump: JASCO PU 2086 Plus x2, detector: JASCO MX-2080-32, degasser: ERC Inc. EC3325, data analysis by JASCO ChromNAV 1.5.2.). Optical rotations were measured on JASCO P-2200 polarimeter at ambient temperature using sodium D line. Melting points were measured on Yanaco MP-J3 micro melting point apparatus, and were uncorrected. Infrared (IR) spectra were recorded on JASCO FT/IR-4100 spectrometer as a thin film on a KBr or ZnSe. The 1D and 2D NMR spectra were recorded on JNM-ECS-400, JCM-ECX-500, or JNM-ECZ-500 spectrometer. Chemical shifts were denoted in ppm on the $\delta$ scale relative to residual solvent peaks as internal standard: $\mathrm{CHCl}_{3}$ ( $\delta=7.26$ for ${ }^{1} \mathrm{H}$ NMR), $\mathrm{CDCl}_{3}$ ( $\delta=77.0$ for $\left.{ }^{13} \mathrm{C} \mathrm{NMR}\right), \mathrm{C}_{6} \mathrm{D}_{5} \mathrm{H}(\delta=7.16$ for $\left.{ }^{1} \mathrm{H} \mathrm{NMR}\right), \mathrm{C}_{6} \mathrm{D}_{6}\left(\delta=128.0\right.$ for $\left.{ }^{13} \mathrm{C} \mathrm{NMR}\right), \mathrm{CO}\left(\mathrm{CD}_{3}\right)\left(\mathrm{CD}_{2} \mathrm{H}\right)\left(\delta=2.05\right.$ for $\left.{ }^{1} \mathrm{H} \mathrm{NMR}\right), \mathrm{CO}\left(\mathrm{CD}_{3}\right)_{2}$ $\left(\delta=29.84\right.$ for ${ }^{13} \mathrm{C}$ NMR), $\mathrm{D}_{2} \mathrm{O}$ (containing acetone as internal reference; $\delta=2.22$ for ${ }^{1} \mathrm{H}$ NMR and $\delta=215.94$ for ${ }^{13} \mathrm{C}$ NMR). Signal patterns are indicated as s, singlet; $\mathrm{d}$, doublet; $\mathrm{t}$, triplet; q, quartet, m; multiplet, br; broaden peak. The carbon numbering of compounds corresponds to that of hikizimycin (1), unless otherwise noted (Figure S1). High resolution mass spectra were measured on JEOL JMS-T100LP (ESI-TOF). 


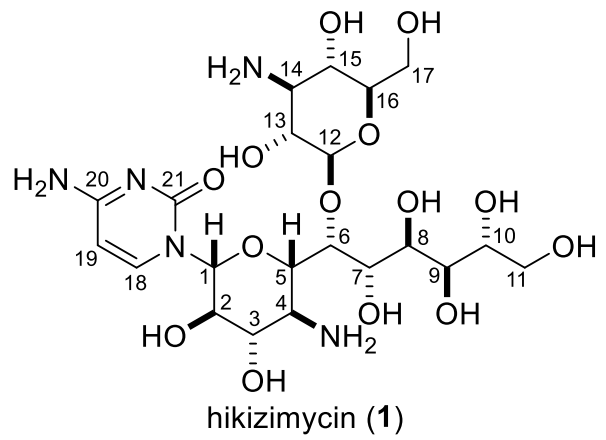

Figure S1. Numbering system of hikizimycin (1)

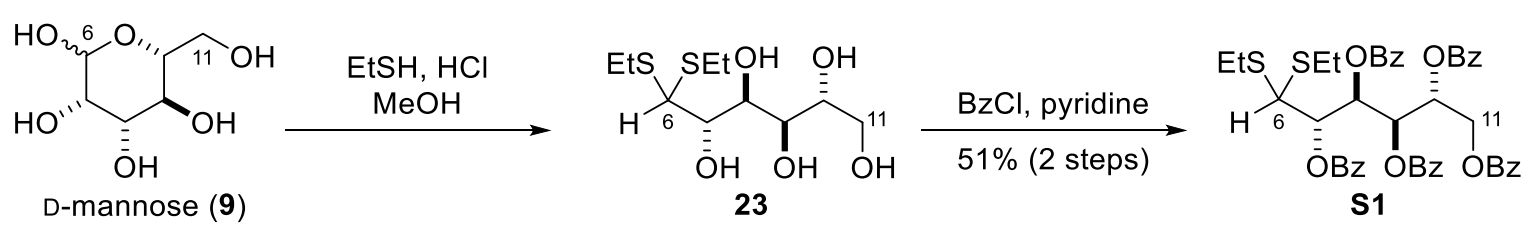

Pentabenzoate S1 [CAS: 103568-91-8]. ${ }^{\mathrm{S} 1}$ A solution of D-mannose (9, $\left.5.40 \mathrm{~g}, 30.0 \mathrm{mmol}\right)$ and ethanethiol $(8.65 \mathrm{~mL}, 120 \mathrm{mmol})$ in $0.5 \mathrm{M} \mathrm{HCl}$ in $\mathrm{MeOH}(60.0 \mathrm{~mL})$ was stirred at $25^{\circ} \mathrm{C}$ for $67 \mathrm{~h}$. The volatile materials were azeotropically removed by using benzene $(50 \mathrm{~mL} \times 3)$ to afford the crude dithioacetal 23 [CAS: $6748-69-2]^{\mathrm{S} 2}(8.74 \mathrm{~g})$, which was used in the next reaction without further purification.

Benzoyl chloride $(\mathrm{BzCl}, 4.65 \mathrm{~mL}, 40.0 \mathrm{mmol}$ ) was added to a solution of the above crude dithioacetal $23(1.46 \mathrm{~g})$ in pyridine $(25.0 \mathrm{~mL})$. After the reaction mixture was stirred at $25^{\circ} \mathrm{C}$ for $17 \mathrm{~h}, \mathrm{H}_{2} \mathrm{O}(30 \mathrm{~mL})$ was added. The resultant mixture was extracted with $\mathrm{Et}_{2} \mathrm{O}(20 \mathrm{~mL} \times 3)$. The combined organic layers were dried over $\mathrm{Na}_{2} \mathrm{SO}_{4}$, concentrated, and azeotroped with benzene $(10 \mathrm{~mL} x 3)$. The residue was purified by flash column chromatography on silica gel $(120 \mathrm{~g}$, hexane/EtOAc $=10 / 1$ to $3 / 1)$ and then by silica gel $(120 \mathrm{~g}$, toluene/EtOAc $=1 / 0$ to $50 / 1)$ to afford pentabenzoate $\mathbf{S 1}(2.05 \mathrm{~g}, 2.54 \mathrm{mmol})$ in $51 \%$ yield over 2 steps as a colorless amorphous, which was used in the next reaction without further purification. $[\alpha]_{\mathrm{D}}{ }^{24}+14.3(c$ 1.48, $\mathrm{CHCl}_{3}$ ); IR (film) 3065, 3035, 2968, 2928, 1727, 1263, $1106 \mathrm{~cm}^{-1} ;{ }^{1} \mathrm{H} \mathrm{NMR} \mathrm{(400} \mathrm{MHz,}$ $\left.\mathrm{CDCl}_{3}\right): \delta 1.19\left(3 \mathrm{H}, \mathrm{t}, J=7.8 \mathrm{~Hz},-\mathrm{SCH}_{2} \mathrm{CH}_{3}\right), 1.27\left(3 \mathrm{H}, \mathrm{t}, J=7.4 \mathrm{~Hz},-\mathrm{SCH}_{2} \mathrm{CH}_{3}\right), 2.57-2.73$ $\left(2 \mathrm{H}, \mathrm{m},-\mathrm{SCH}_{2} \mathrm{CH}_{3}\right), 2.74-2.88\left(2 \mathrm{H}, \mathrm{m},-\mathrm{SCH}_{2} \mathrm{CH}_{3}\right), 4.49(1 \mathrm{H}, \mathrm{dd}, J=12.8,5.0 \mathrm{~Hz}, \mathrm{H} 11 \mathrm{a}), 4.50$ $(1 \mathrm{H}, \mathrm{d}, J=8.2 \mathrm{~Hz}, \mathrm{H} 6), 4.85$ (1H, dd, $J=12.8,3.2 \mathrm{~Hz}, \mathrm{H} 11 \mathrm{~b}), 5.77$ (1H, ddd, $J=8.2,5.0,3.2$ Hz, H10), 5.87 (1H, dd, $J=8.2,5.0 \mathrm{~Hz}, \mathrm{H} 7), 6.30(1 \mathrm{H}, \mathrm{dd}, J=5.0,1.4 \mathrm{~Hz}, \mathrm{H} 8), 6.36(1 \mathrm{H}, \mathrm{dd}$, $J=8.2,1.4 \mathrm{~Hz}, \mathrm{H} 9), 7.06$ (2H, t, $J=8.2 \mathrm{~Hz}$, aromatic), 7.34-7.42 (9H, m, aromatic), 7.51-7.55 (4H, m, aromatic), $7.67(2 \mathrm{H}, \mathrm{d}, J=7.3 \mathrm{~Hz}$, aromatic), 7.96-8.03 (8H, m, aromatic); Detectable signals of ${ }^{13} \mathrm{C} \mathrm{NMR}\left(100 \mathrm{MHz}, \mathrm{CDCl}_{3}\right)$ : $\delta 14.1,14.2,24.4,24.9,51.7,62.4,68.1,69.6,70.0$, 
$72.6,127.9,128.3,128.4,128.5,129.0,129.1,129.27,129.28,129.30,129.5,129.65,129.73$, 129.76, 129.83, 129.86, 129.90, 132.8, 133.0, 133.2, 133.3, 133.4, 165.20, 165.24. 165.3, 165.4, 165.9; HRMS (ESI-TOF) calcd for $\mathrm{C}_{45} \mathrm{H}_{42} \mathrm{O}_{10} \mathrm{~S}_{2} \mathrm{Na}[\mathrm{M}+\mathrm{Na}]^{+} 829.2112$, found 829.2085.

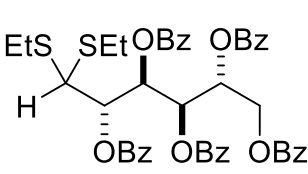

S1

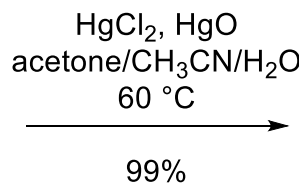

$99 \%$

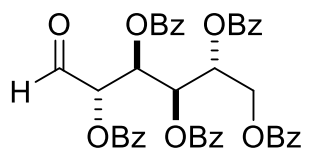

$7 \mathrm{a}$

Aldehyde 7a. $\mathrm{HgCl}_{2}(153 \mathrm{mg}, 565 \mu \mathrm{mol})$ and $\mathrm{HgO}(245 \mathrm{mg}, 1.13 \mathrm{mmol})$ was added to a solution of dithioacetal $\mathbf{S 1}(228 \mathrm{mg}, 283 \mu \mathrm{mol})$ in acetone $(2.83 \mathrm{~mL}), \mathrm{CH}_{3} \mathrm{CN}(2.83 \mathrm{~mL})$, and $\mathrm{H}_{2} \mathrm{O}(1.41 \mathrm{~mL})$ at $25^{\circ} \mathrm{C}$. After being vigorously stirred at $60^{\circ} \mathrm{C}$ for $6 \mathrm{~h}$, the reaction mixture was filtered through a pad of Celite with $\mathrm{CH}_{2} \mathrm{Cl}_{2}(4 \mathrm{~mL})$. The volume of the filtrate was then reduced to $2 \mathrm{~mL}$ by using a rotary evaporator before addition of $20 \%$ aqueous $\mathrm{KI}(4 \mathrm{~mL})$ to the mixture. The resultant mixture was extracted with $\mathrm{CH}_{2} \mathrm{Cl}_{2}(5 \mathrm{~mL} \times 3)$. The combined organic layers were dried over $\mathrm{Na}_{2} \mathrm{SO}_{4}$ and concentrated. The residue was filtered through a short pad of silica gel (Fuji Silysia, $10 \mathrm{~g}$, hexane/EtOAc $=1 / 2)$ to afford aldehyde 7a $(229 \mathrm{mg}$, $283 \mu \mathrm{mol})$ in $99 \%$ yield as a colorless amorphous, which was used immediately in the next reaction. ${ }^{1} \mathrm{H}$ NMR (400 MHz, $\left.\mathrm{C}_{6} \mathrm{D}_{6}\right): \delta 4.40(1 \mathrm{H}, \mathrm{m}, \mathrm{H} 11 \mathrm{a}), 4.95(1 \mathrm{H}, \mathrm{dd}, J=12.4,2.8 \mathrm{~Hz}$, H11b), $5.81(1 \mathrm{H}, \mathrm{m}, \mathrm{H} 10), 6.10(1 \mathrm{H}, \mathrm{m}, \mathrm{H} 7), 6.53$ (1H, dd, $J=6.0,2.8 \mathrm{~Hz}, \mathrm{H} 8), 6.59$ (1H, dd, $J=7.8,3.2 \mathrm{~Hz}, \mathrm{H} 9), 6.85-7.05(15 \mathrm{H}, \mathrm{m}$, aromatic $), 7.98-8.18(10 \mathrm{H}, \mathrm{m}$, aromatic $), 9.36(1 \mathrm{H}, \mathrm{m}$, H6).

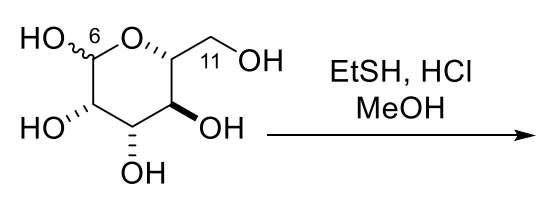

D-mannose (9)

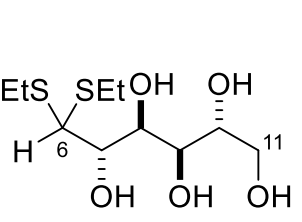

23

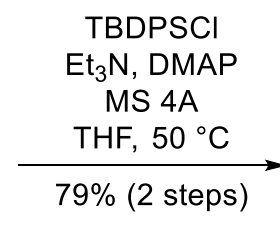

(1)

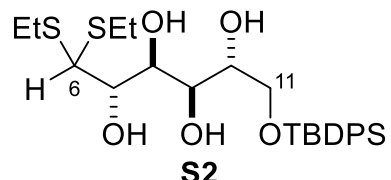

S2

Tetraol S2 [Reaxys ID: 6942683]. ${ }^{\mathrm{S} 3} \quad$ A solution of D-mannose $(\mathbf{9}, 6.00 \mathrm{~g}, 33.3 \mathrm{mmol})$ and ethanethiol $(9.61 \mathrm{~mL}, 133 \mathrm{mmol})$ in $0.5 \mathrm{M} \mathrm{HCl}$ in $\mathrm{MeOH}(66.6 \mathrm{~mL})$ was stirred at $25^{\circ} \mathrm{C}$ for 70 h. The volatile materials were azeotropically removed by using benzene $(50 \mathrm{~mL} \times 3)$ to afford the crude dithioacetal $\mathbf{2 3}$, which was used in the next reaction without further purification. $t$-Butyldiphenylchlorosilane (TBDPSCl, $43.3 \mathrm{~mL}, 167 \mathrm{mmol}$ ) was added to a slurry of the above crude dithioacetal 23, $\mathrm{Et}_{3} \mathrm{~N}(37.1 \mathrm{~mL}, 266 \mathrm{mmol})$, 4-dimethylaminopyridine (DMAP, $203 \mathrm{mg}, 1.67 \mathrm{mmol})$, and molecular sieves $4 \mathrm{~A}(15.3 \mathrm{~g})$ in THF $(111 \mathrm{~mL})$ at $25^{\circ} \mathrm{C}$. After being stirred at $25{ }^{\circ} \mathrm{C}$ for $48 \mathrm{~h}$, the reaction mixture was filtered through a pad of Celite with 
$\mathrm{CHCl}_{3} / \mathrm{MeOH}(2 / 1,30 \mathrm{~mL})$. After saturated aqueous $\mathrm{NH}_{4} \mathrm{Cl}(50 \mathrm{~mL})$ was added to the filtrate, the resultant mixture was extracted with $\mathrm{CHCl}_{3} / \mathrm{MeOH}(2 / 1,20 \mathrm{~mL}$ x3). The combined organic layers were dried over $\mathrm{Na}_{2} \mathrm{SO}_{4}$ and concentrated. The residue was purified by flash column chromatography on silica gel $\left(300 \mathrm{~g}, \mathrm{CH}_{2} \mathrm{Cl}_{2} / \mathrm{MeOH}=20 / 1\right)$ to afford tetraol $\mathbf{S 2}(13.8$ g, $26.3 \mathrm{mmol}$ ) in $79 \%$ yield as colorless oil. The analytical data of $\mathbf{S 2}$ were identical to those reported previously.

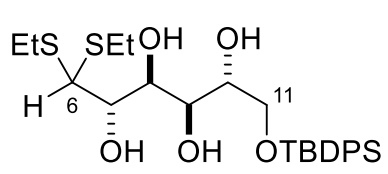

s2

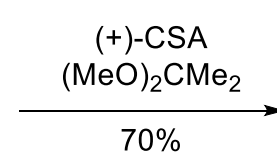

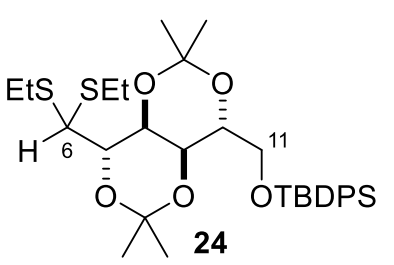

24

Bis-acetonide 24 [CAS: 155809-03-3]. ${ }^{\mathrm{S} 3} \quad(+)$-Camphorsulfonic acid ((+)-CSA, $34.5 \mathrm{mg}, 149$ mmol) was added to a solution of tetraol $\mathbf{S 2}(1.56 \mathrm{~g}, 2.97 \mathrm{mmol})$ in $(\mathrm{MeO})_{2} \mathrm{CMe}_{2}(20 \mathrm{~mL})$ at $25^{\circ} \mathrm{C}$. The reaction mixture was stirred at $25^{\circ} \mathrm{C}$ for $22 \mathrm{~h}$. After $\mathrm{Et}_{3} \mathrm{~N}(5 \mathrm{~mL})$ was added, the resultant mixture was concentrated. The residue was purified by flash column chromatography on silica gel $(50 \mathrm{~g}$, hexane/EtOAc $=100 / 1$ to $40 / 1)$ to afford bis-acetonide 24 $(1.26 \mathrm{~g}, 2.08 \mathrm{mmol})$ in $70 \%$ yield as colorless solid. The analytical data of $\mathbf{2 4}$ were identical to those reported previously.
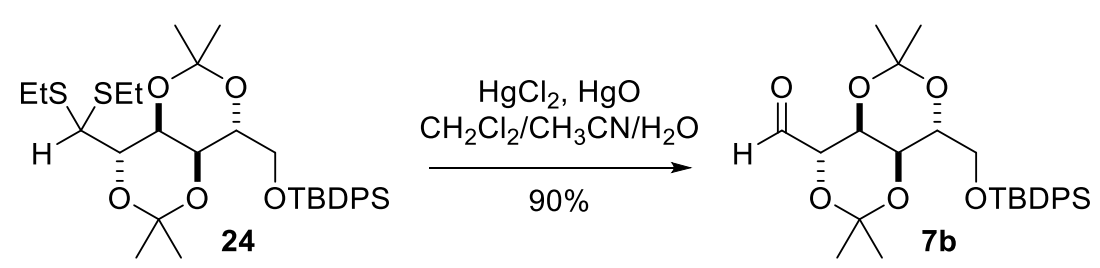

Aldehyde 7b [CAS: 154796-66-4]. ${ }^{\mathrm{S} 3} \mathrm{HgCl}_{2}(212 \mathrm{mg}, 780 \mu \mathrm{mol})$ and $\mathrm{HgO}(338 \mathrm{mg}, 1.56$ mmol) was added to a solution of dithioacetal 24 (236 mg, $390 \mu \mathrm{mol})$ in $\mathrm{CH}_{2} \mathrm{Cl}_{2}(2.60 \mathrm{~mL})$, $\mathrm{CH}_{3} \mathrm{CN}(4.33 \mathrm{~mL})$, and $\mathrm{H}_{2} \mathrm{O}(867 \mu \mathrm{L})$ at $25^{\circ} \mathrm{C}$. After being vigorously stirred at $25^{\circ} \mathrm{C}$ for 30 min, the reaction mixture was filtered through a pad of Celite with $\mathrm{CH}_{2} \mathrm{Cl}_{2}(4 \mathrm{~mL})$. The volume of the filtrate was then reduced to $2 \mathrm{~mL}$ by using a rotary evaporator before addition of $20 \%$ aqueous $\mathrm{KI}(4 \mathrm{~mL})$ to the mixture. The resultant mixture was extracted with $\mathrm{CH}_{2} \mathrm{Cl}_{2}(5$ $\mathrm{mL} \times 3)$. The combined organic layers were dried over $\mathrm{Na}_{2} \mathrm{SO}_{4}$ and concentrated. The residue was purified by flash column chromatography on silica gel (Fuji Silysia, $20 \mathrm{~g}$, hexane $/$ EtOAc $=25 / 1$ to $5 / 1)$ to afford aldehyde $7 \mathbf{b}(176 \mathrm{mg}, 353 \mu \mathrm{mol})$ in $90 \%$ yield: colorless amorphous; ${ }^{1} \mathrm{H}$ NMR (400 MHz, $\left.\mathrm{C}_{6} \mathrm{D}_{6}\right): \delta 1.14\left(3 \mathrm{H}, \mathrm{s}, \mathrm{CH}_{3}\right.$ of acetonide), $1.18\left(9 \mathrm{H}, \mathrm{s}, \mathrm{C}\left(\mathrm{CH}_{3}\right)_{3}\right.$ of TBDPS), $1.20\left(3 \mathrm{H}, \mathrm{s}, \mathrm{CH}_{3}\right.$ of acetonide), $1.30\left(3 \mathrm{H}, \mathrm{s}, \mathrm{CH}_{3}\right.$ of acetonide), $1.35\left(3 \mathrm{H}, \mathrm{s}, \mathrm{CH}_{3}\right.$ of 
acetonide), $3.82(1 \mathrm{H}, \mathrm{dd}, J=8.2,4.1 \mathrm{~Hz}, \mathrm{H} 9), 3.86-3.87(2 \mathrm{H}, \mathrm{m}, \mathrm{H} 11), 3.97-4.04$ (2H, m, H8, H10), 4.07 (1H, d, $J=9.2 \mathrm{~Hz}, \mathrm{H} 7), 7.21-7.24$ (6H, m, aromatic), 7.81-7.86 (4H, m, aromatic), $9.48(1 \mathrm{H}, \mathrm{s}, \mathrm{CHO})$. The analytical data of $\mathbf{7 b}$ were identical to those reported previously.

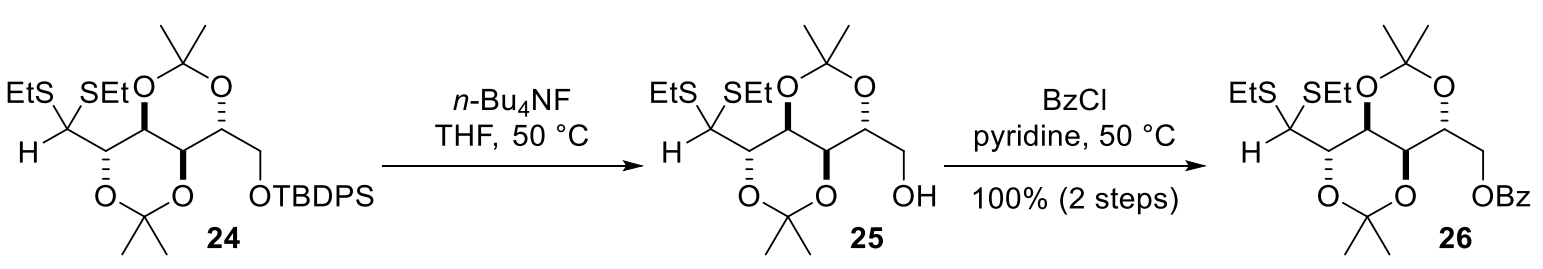

Benzoate 26. $n$-Bu4NF (1.0 M THF solution, $19.2 \mathrm{~mL}, 19.2 \mathrm{mmol}$ ) was added to a solution of dithioacetal $24(7.72 \mathrm{~g}, 12.8 \mathrm{mmol})$ in THF $(64.0 \mathrm{~mL})$ at $25^{\circ} \mathrm{C}$. After the reaction mixture was stirred at $50{ }^{\circ} \mathrm{C}$ for $2 \mathrm{~h}, \mathrm{H}_{2} \mathrm{O}(60 \mathrm{~mL})$ was added to the mixture. The resultant mixture was extracted with EtOAc $(60 \mathrm{~mL} \times 3)$, dried over $\mathrm{Na}_{2} \mathrm{SO}_{4}$, and concentrated. The residue was purified by a short pad of silica gel $(200 \mathrm{~g}$, hexane/EtOAc $=15 / 1$ to $1 / 1)$ to afford the crude alcohol $25(6.10 \mathrm{~g})$, which was used in the next reaction without further purification: colorless oil.

$\mathrm{BzCl}(2.97 \mathrm{~mL}, 25.6 \mathrm{mmol})$ was added to a solution of the above crude alcohol $25(6.10 \mathrm{~g})$ in pyridine $(64.0 \mathrm{~mL})$ at $25^{\circ} \mathrm{C}$. After the reaction mixture was stirred at $50{ }^{\circ} \mathrm{C}$ for $14 \mathrm{~h}, \mathrm{H}_{2} \mathrm{O}(60$ $\mathrm{mL})$ was added. The resultant mixture was extracted with $\mathrm{CH}_{2} \mathrm{Cl}_{2}(60 \mathrm{~mL} \times 3)$, dried over $\mathrm{Na}_{2} \mathrm{SO}_{4}$, and concentrated. The residue was purified by flash column chromatography on amine-modified silica gel $(600 \mathrm{~g}$, hexane/EtOAc $=50 / 1)$ and then on silica gel $(600 \mathrm{~g}$, hexane $/$ EtOAc $=50 / 1$ to 20/1) to afford benzoate $26(6.04 \mathrm{~g}, 12.8 \mathrm{mmol})$ in $100 \%$ yield over 2 steps: colorless oil; $[\alpha]_{\mathrm{D}}{ }^{25}+10.6\left(c 0.73, \mathrm{CHCl}_{3}\right)$; IR (film) 2986, 2928, 2872, 1724, 1378, 1275 , 1218, $1107 \mathrm{~cm}^{-1} ;{ }^{1} \mathrm{H} \mathrm{NMR}\left(400 \mathrm{MHz}, \mathrm{CDCl}_{3}\right): \delta 1.26\left(3 \mathrm{H}, \mathrm{t}, J=7.8 \mathrm{~Hz},-\mathrm{SCH}_{2} \mathrm{CH}_{3}\right), 1.27(3 \mathrm{H}$, t, $\left.J=7.4 \mathrm{~Hz},-\mathrm{SCH}_{2} \mathrm{CH}_{3}\right), 1.29\left(3 \mathrm{H}, \mathrm{s}, \mathrm{CH}_{3}\right.$ of acetonide), 1.37 (3H, s, $\mathrm{CH}_{3}$ of acetonide), 1.38 ( $3 \mathrm{H}, \mathrm{s}, \mathrm{CH}_{3}$ of acetonide), $1.43\left(3 \mathrm{H}, \mathrm{s}, \mathrm{CH}_{3}\right.$ of acetonide), 2.66-2.79 (4H, m, $-\mathrm{SCH}_{2} \mathrm{CH}_{3} \mathrm{x} 2$ ), 3.90-3.95 (2H, m, H8, H9), 4.02-4.06 (2H, m, H7, H10), 4.33-4.40 (2H, H6, H11a), 4.47 (1H, $\mathrm{dd}, J=11.9 \mathrm{~Hz}, \mathrm{H} 11 \mathrm{~b}), 7.44(2 \mathrm{H}, \mathrm{t}, J=7.8 \mathrm{~Hz}$, aromatic), 7.57 (1H, t, $J=7.8 \mathrm{~Hz}$, aromatic), $8.04\left(2 \mathrm{H}, \mathrm{d}, J=7.8 \mathrm{~Hz}\right.$, aromatic); ${ }^{13} \mathrm{C} \mathrm{NMR}\left(100 \mathrm{MHz}, \mathrm{CDCl}_{3}\right): \delta 14.4,14.7,23.6,23.8,24.4$, 24.5, 25.2, 25.3, 52.7, 64.8, 68.2, 68.6, 69.4, 74.4, 101.1, 101.4, 128.3 (2C), 129.6 (2C), 130.0, 133.0, 166.4; HRMS (ESI-TOF) calcd for $\mathrm{C}_{23} \mathrm{H}_{34} \mathrm{O}_{6} \mathrm{~S}_{2} \mathrm{Na}[\mathrm{M}+\mathrm{Na}]^{+} 493.1689$, found 493.1679 . 


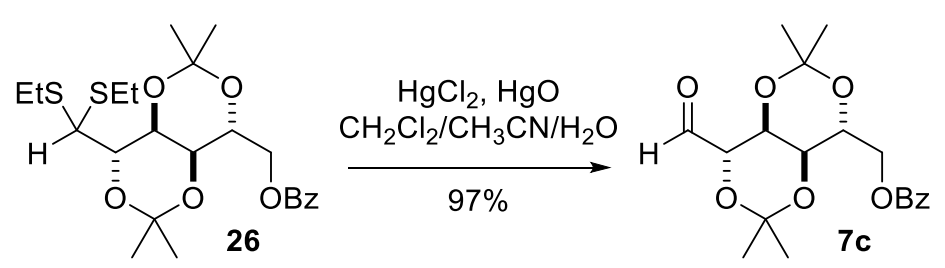

Aldehyde 7c. $\mathrm{HgCl}_{2}(1.89 \mathrm{~g}, 6.96 \mathrm{mmol})$ and $\mathrm{HgO}(2.40 \mathrm{~g}, 11.1 \mathrm{mmol})$ was added to a solution of dithioacetal 26 (1.64 g, $3.48 \mathrm{mmol})$ in $\mathrm{CH}_{2} \mathrm{Cl}_{2}(23.2 \mathrm{~mL}), \mathrm{CH}_{3} \mathrm{CN}(38.7 \mathrm{~mL})$, and $\mathrm{H}_{2} \mathrm{O}(7.73 \mathrm{~mL})$ at $25^{\circ} \mathrm{C}$. After being vigorously stirred at $25^{\circ} \mathrm{C}$ for $1 \mathrm{~h}$, the reaction mixture was filtered through a pad of Celite with $\mathrm{CH}_{2} \mathrm{Cl}_{2}(100 \mathrm{~mL})$. Then, $40 \%$ aqueous $\mathrm{KI}(50 \mathrm{~mL})$ was added to the filtrate. The resultant mixture was extracted with $\mathrm{CH}_{2} \mathrm{Cl}_{2}(50 \mathrm{~mL} \times 3)$. The combined organic layers were washed with $40 \%$ aqueous $\mathrm{KI}(50 \mathrm{~mL} \times 3)$ and saturated aqueous $\mathrm{NaHCO}_{3}(100 \mathrm{~mL})$, dried over $\mathrm{Na}_{2} \mathrm{SO}_{4}$, and concentrated. The residue was purified by flash column chromatography on silica gel (Fuji Silysia, $120 \mathrm{~g}$, hexane/EtOAc $=10 / 1$ to 3/2) to afford aldehyde $7 \mathrm{c}(1.23 \mathrm{~g}, 3.38 \mathrm{mmol})$ in 97\% yield: colorless amorphous; $[\alpha]_{\mathrm{D}}{ }^{24}+14.3$ (c 1.08 , $\mathrm{CHCl}_{3}$ ); IR (film) 3471, 2988, 2939, 1381, 1277, 1220, 1173, 1110, $714 \mathrm{~cm}^{-1} ;{ }^{1} \mathrm{H}$ NMR (400 $\left.\mathrm{MHz}, \mathrm{C}_{6} \mathrm{D}_{6}\right): \delta 1.11\left(3 \mathrm{H}, \mathrm{s}, \mathrm{CH}_{3}\right.$ of acetonide), $1.16\left(3 \mathrm{H}, \mathrm{s}, \mathrm{CH}_{3}\right.$ of acetonide), $1.25\left(3 \mathrm{H}, \mathrm{s}, \mathrm{CH}_{3}\right.$ of acetonide), 1.29 (3H, s, $\mathrm{CH}_{3}$ of acetonide), $3.67(1 \mathrm{H}, \mathrm{dd}, J=8.7,4.6 \mathrm{~Hz}, \mathrm{H} 9), 3.97(1 \mathrm{H}, \mathrm{dd}$, $J=8.7,4.6 \mathrm{~Hz}, \mathrm{H} 8), 4.02(1 \mathrm{H}, \mathrm{d}, J=8.7 \mathrm{~Hz}, \mathrm{H} 7), 4.13$ (1H, ddd, $J=8.7,6.9,3.7 \mathrm{~Hz}, \mathrm{H} 10)$, $4.43(1 \mathrm{H}, \mathrm{dd}, J=11.9,3.7 \mathrm{~Hz}, \mathrm{H} 11 \mathrm{a}), 4.50(1 \mathrm{H}, \mathrm{dd}, J=11.9,6.9 \mathrm{~Hz}, \mathrm{H} 11 \mathrm{~b}) 7.04$ (2H, t, $J=7.8$ $\mathrm{Hz}$, aromatic), $7.10(1 \mathrm{H}, \mathrm{d}, J=7.8 \mathrm{~Hz}$, aromatic), $8.17(2 \mathrm{H}, \mathrm{t}, J=7.8 \mathrm{~Hz}$, aromatic), $9.44(1 \mathrm{H}$, s, $\mathrm{CHO}) ;{ }^{13} \mathrm{C}$ NMR $\left(100 \mathrm{MHz}, \mathrm{C}_{6} \mathrm{D}_{6}\right): \delta 23.4,23.7,24.2,24.3,64.7,67.4,68.7,69.0,73.9$, 101.4, 101.5, 128.5 (2C), 129.9 (2C), 130.7, 133.0, 166.1, 196.8; HRMS (ESI-TOF) calcd for $\mathrm{C}_{19} \mathrm{H}_{24} \mathrm{O}_{7} \mathrm{Na}[\mathrm{M}+\mathrm{Na}]^{+}$387.1414, found 387.1415.
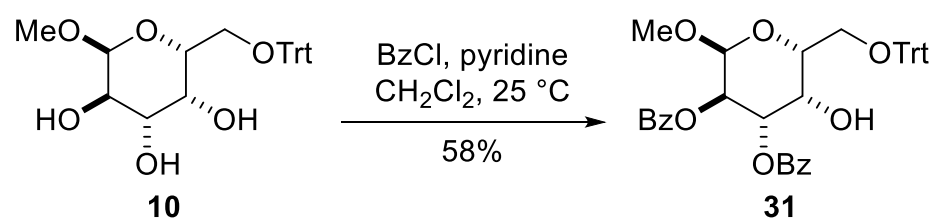

Alcohol 31 [CAS: 31505-55-2]. ${ }^{\mathrm{S} 4} \mathrm{BzCl}(10.7 \mathrm{~mL}, 92.0 \mathrm{mmol})$ was added to a solution of triol 10 (16.1 g, $36.8 \mathrm{mmol}$ ) and pyridine $(11.8 \mathrm{~mL}, 147 \mathrm{mmol})$ in $\mathrm{CH}_{2} \mathrm{Cl}_{2}(350 \mathrm{~mL})$ at $25^{\circ} \mathrm{C}$. After the reaction mixture was stirred for $3 \mathrm{~h}$ at $25^{\circ} \mathrm{C}, \mathrm{H}_{2} \mathrm{O}(100 \mathrm{~mL})$ was added at $25^{\circ} \mathrm{C}$. The organic layer was separated and the aqueous layer was extracted with EtOAc $(50 \mathrm{~mL} \times 3)$. The combined organic layers were washed with $1 \mathrm{M}$ aqueous $\mathrm{HCl}(50 \mathrm{~mL})$, saturated aqueous $\mathrm{NaHCO}_{3}(50 \mathrm{~mL})$, and brine $(50 \mathrm{~mL})$. The resultant organic layers were dried over $\mathrm{Na}_{2} \mathrm{SO}_{4}$, filtered, and concentrated. The residue was purified by flash column chromatography on 
silica gel (600 g, hexane/EtOAc $=5 / 1$ to $3 / 1)$ to afford alcohol $31(13.9 \mathrm{~g}, 21.5 \mathrm{mmol})$ in 58\% yield as colorless solid. The analytical data of $\mathbf{3 1}$ were identical to those reported previously.

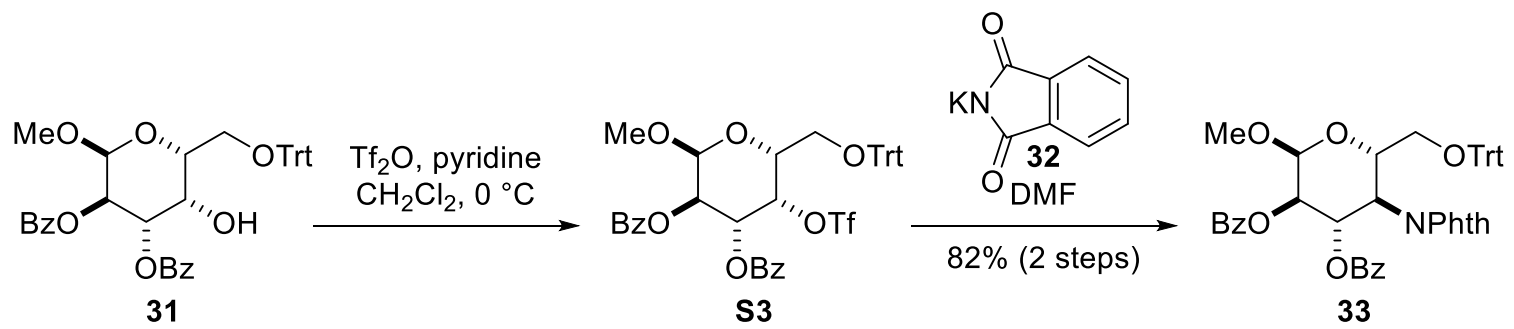

Phthalimide 33. Trifluoromethanesulfonic anhydride $\left(\mathrm{Tf}_{2} \mathrm{O}, 5.30 \mathrm{~mL}, 32.3 \mathrm{mmol}\right)$ was slowly added to a solution of alcohol 31 (13.9 g, $21.5 \mathrm{mmol})$ and pyridine ( $8.65 \mathrm{~mL}, 108 \mathrm{mmol})$ in $\mathrm{CH}_{2} \mathrm{Cl}_{2}(71.3 \mathrm{~mL})$ at $0{ }^{\circ} \mathrm{C}$. The reaction mixture was stirred for $30 \mathrm{~min}$ at $0{ }^{\circ} \mathrm{C}$, and then $\mathrm{H}_{2} \mathrm{O}(120 \mathrm{~mL})$ was added. The resultant mixture was extracted with $\mathrm{CH}_{2} \mathrm{Cl}_{2}(90 \mathrm{~mL} \times 3)$. The combined organic layers were washed with brine $(200 \mathrm{~mL})$, dried over $\mathrm{Na}_{2} \mathrm{SO}_{4}$, and concentrated to afford the crude triflate $\mathbf{S 3}$ (16.4 g), which was used in the next reaction without further purification.

Potassium phthalimide $(32,7.97 \mathrm{~g}, 43.0 \mathrm{mmol})$ was added to a solution of the above crude triflate S3 $(16.4 \mathrm{~g})$ in DMF $(75 \mathrm{~mL})$ at $25^{\circ} \mathrm{C}$. After the reaction mixture was stirred at $25{ }^{\circ} \mathrm{C}$ for $14 \mathrm{~h}, \mathrm{H}_{2} \mathrm{O}(200 \mathrm{~mL})$ was added. The resultant mixture was extracted with hexane/EtOAc $(1 / 1,200 \mathrm{~mL} \times 3)$. The combined organic layers were washed with brine $(200 \mathrm{~mL})$, dried over $\mathrm{Na}_{2} \mathrm{SO}_{4}$, and concentrated. The residue was purified by flash column chromatography on silica gel $(400 \mathrm{~g}$, hexane/EtOAc $=5 / 1$ to $3 / 1)$ to afford phthalimide $33(13.7 \mathrm{~g}, 17.6 \mathrm{mmol})$ in $82 \%$ yield over 2 steps: colorless solid; m.p. $128-131{ }^{\circ} \mathrm{C}$; $[\alpha]_{\mathrm{D}}{ }^{25}+11.3\left(\right.$ c $\left.1.20, \mathrm{CHCl}_{3}\right)$; IR (film) 3060, 3031, 2937, 1777, 1723, 1379, $1273 \mathrm{~cm}^{-1} ;{ }^{1} \mathrm{H}$ NMR (400 MHz, $\mathrm{CDCl}_{3}$ ): $\delta 3.07$ $(1 \mathrm{H}, \mathrm{dd}, J=11.0,4.1 \mathrm{~Hz}, \mathrm{H} 6 \mathrm{a}), 3.32(1 \mathrm{H}, \mathrm{dd}, J=11.0,3.2 \mathrm{~Hz}, \mathrm{H} 6 \mathrm{~b}), 3.49$ (3H, s, OCH $), 4.76$ $(1 \mathrm{H}, \mathrm{dd}, J=10.0,10.0 \mathrm{~Hz}, \mathrm{H} 4), 7.10$ (1H, ddd, $J=10.0,4.1,3.2 \mathrm{~Hz}, \mathrm{H} 5), 5.28(1 \mathrm{H}, \mathrm{d}, J=3.6$ Hz, H1), 5.37 (1H, dd, $J=10.0,3.6 \mathrm{~Hz}, \mathrm{H} 2), 6.47$ (1H, dd, $J=10.0,10.0 \mathrm{~Hz}, \mathrm{H} 3), 7.05-7.15$ $(8 \mathrm{H}, \mathrm{m}$, aromatic), 7.24-7.28 $(3 \mathrm{H}, \mathrm{m}$, aromatic), 7.33-7.42 $(9 \mathrm{H}, \mathrm{m}$, aromatic $), 7.50(1 \mathrm{H}, \mathrm{m}$, aromatic), 7.60-7.65 (3H, m, aromatic), $7.74(1 \mathrm{H}, \mathrm{d}, J=7.3 \mathrm{~Hz}$, aromatic), $7.79(2 \mathrm{H}, \mathrm{d}, J=7.8$ $\mathrm{Hz}$, aromatic), $8.00\left(2 \mathrm{H}, \mathrm{d}, J=7.8 \mathrm{~Hz}\right.$, aromatic); $\left.{ }^{13} \mathrm{C} \mathrm{NMR} \mathrm{(100} \mathrm{MHz,} \mathrm{CDCl}_{3}\right): \delta 51.9,55.4$, 63.0, 65.9, 68.7, 73.0, 86.7, 97.1, 123.3, 123.4, 126.8 (3C), $127.6(6 \mathrm{C}), 128.2(2 \mathrm{C}), 128.3(2 \mathrm{C})$, 128.5 (6C), 129.0, 129.1, 129.6 (2C), 129.9 (2C), 131.2, 131.5, 133.0, 133.3, 133.7, 133.9, 143.4 (3C), 165.7, 165.9, 166.9, 167.8; HRMS (ESI-TOF) calcd for $\mathrm{C}_{48} \mathrm{H}_{39} \mathrm{NO}_{9} \mathrm{Na}[\mathrm{M}+\mathrm{Na}]^{+}$ 796.2517 , found 796.2513 . 


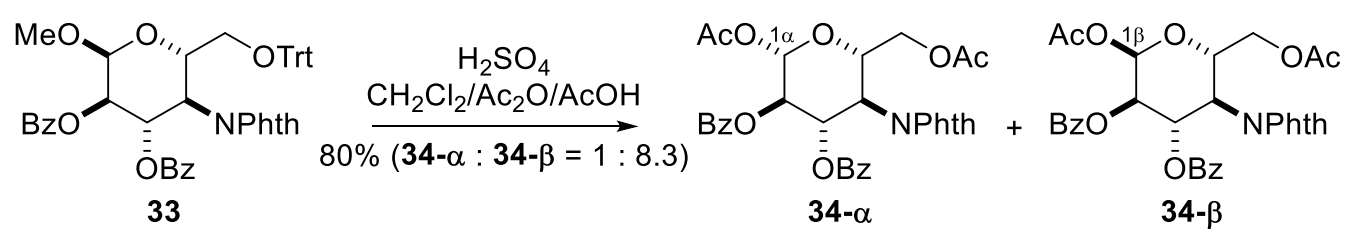

Acetylacetals $34-\boldsymbol{\alpha}$ and $34-\boldsymbol{\beta} . \quad \mathrm{H}_{2} \mathrm{SO}_{4}(648 \mathrm{mg}, 3.50 \mathrm{mmol})$ was added to a solution of phthalimide 33 (1.85 g, $2.39 \mathrm{mmol})$ in $\mathrm{CH}_{2} \mathrm{Cl}_{2}(3.41 \mathrm{~mL}), \mathrm{Ac}_{2} \mathrm{O}(13.7 \mathrm{~mL})$, and $\mathrm{AcOH}(6.83$ $\mathrm{mL}$ ) at $25{ }^{\circ} \mathrm{C}$. After the reaction mixture was stirred at $25^{\circ} \mathrm{C}$ for $19 \mathrm{~h}$, saturated aqueous $\mathrm{NaHCO}_{3}(30 \mathrm{~mL})$ was added at $0{ }^{\circ} \mathrm{C}$. The resultant mixture was extracted with EtOAc (30 $\mathrm{mL} \times 3)$. The combined organic layers were washed with brine $(40 \mathrm{~mL})$, dried over $\mathrm{Na}_{2} \mathrm{SO}_{4}$, and concentrated. The residue was purified by flash column chromatography on silica gel (30 $\mathrm{g}$, hexane/EtOAc $=3 / 1$ ) to afford a $1: 8.3$ diastereomeric mixture of acetylacetal 34- $\alpha$ and 34$\boldsymbol{\beta}(1.14 \mathrm{~g}, 1.90 \mathrm{mmol})$ in 80\% yield: colorless solid; IR (film) 3060, 3031, 2937, 1777, 1723, 1379, $1273 \mathrm{~cm}^{-1}$; ${ }^{1} \mathrm{H}$ NMR (400 MHz, $\left.\mathrm{CDCl}_{3}\right): \delta 2.05\left(3.00 \mathrm{H}, \mathrm{s}, \mathrm{CH} \mathrm{H}_{3} \mathrm{CO}\right), 2.27(3.00 \mathrm{H}, \mathrm{s}$, $\left.\mathrm{CH}_{3} \mathrm{CO}\right), 4.11(0.89 \mathrm{H}, \mathrm{dd}, J=12.8,2.3 \mathrm{~Hz}, \mathrm{H} 6 \mathrm{a}), 4.13(0.11 \mathrm{H}, \mathrm{dd}, J=12.8,2.3 \mathrm{~Hz}, \mathrm{H} 6 \mathrm{a}), 4.32$ $(1.00 \mathrm{H}, \mathrm{dd}, J=12.8,3.6 \mathrm{~Hz}, \mathrm{H} 6 \mathrm{~b}), 4.78(0.89 \mathrm{H}, \mathrm{dd}, J=10.6,10.6 \mathrm{~Hz}, \mathrm{H} 4), 4.78(0.11 \mathrm{H}, \mathrm{dd}, \mathrm{J}$ $=10.5,10.5 \mathrm{~Hz}, \mathrm{H} 4), 4.99(0.11 \mathrm{H}, \mathrm{ddd}, J=10.5,3.6,2.3 \mathrm{~Hz}, \mathrm{H} 5), 5.19$ (0.89H, ddd, $J=10.6$, 3.6, 2.3 Hz, H5), $5.56(0.89 \mathrm{H}, \mathrm{dd}, J=10.6,3.7 \mathrm{~Hz}, \mathrm{H} 2), 5.67(0.11 \mathrm{H}, \mathrm{dd}, J=10.5,8.7 \mathrm{~Hz}, \mathrm{H} 2)$, $6.14(0.11 \mathrm{H}, \mathrm{d}, J=8.7 \mathrm{~Hz}, \mathrm{H} 1), 6.28(0.11 \mathrm{H}, \mathrm{dd}, J=10.5,10.5 \mathrm{~Hz}, \mathrm{H} 3), 6.52(0.89 \mathrm{H}, \mathrm{dd}, J=$ 10.6, 10.6 Hz, H3), $6.61(0.89 \mathrm{H}, \mathrm{d}, J=3.7 \mathrm{~Hz}, \mathrm{H} 1), 7.26-7.30$ (2.00H, m, aromatic), 7.34-7.38 $(2.00 \mathrm{H}, \mathrm{m}$, aromatic), $7.43(1.00 \mathrm{H}, \mathrm{t}, J=7.8 \mathrm{~Hz}$, aromatic), $7.50(1.00 \mathrm{H}, \mathrm{t}, J=7.8 \mathrm{~Hz}$, aromatic), 7.65-7.79 $\left(5.00 \mathrm{H}, \mathrm{m}\right.$, aromatic), 7.89-7.99 $\left(3.00 \mathrm{H}, \mathrm{m}\right.$, aromatic); Detectable signals of ${ }^{13} \mathrm{C}$ NMR (100 MHz, $\left.\mathrm{CDCl}_{3}\right): \delta 20.6,20.9,50.4,62.0,67.2,68.0,70.6,70.8,89.3,123.6,123.7$, $128.3,128.4,128.5,128.6,129.66,129.73,130.9$, 131.4, 133.3, 133.5, 134.4, 134.5, 165.3, 165.6, 167.0, 168.1, 168.9, 170.6; HRMS (ESI-TOF) calcd for $\mathrm{C}_{32} \mathrm{H}_{27} \mathrm{NO}_{11} \mathrm{Na}[\mathrm{M}+\mathrm{Na}]^{+}$ 624.1476 , found 624.1499 .

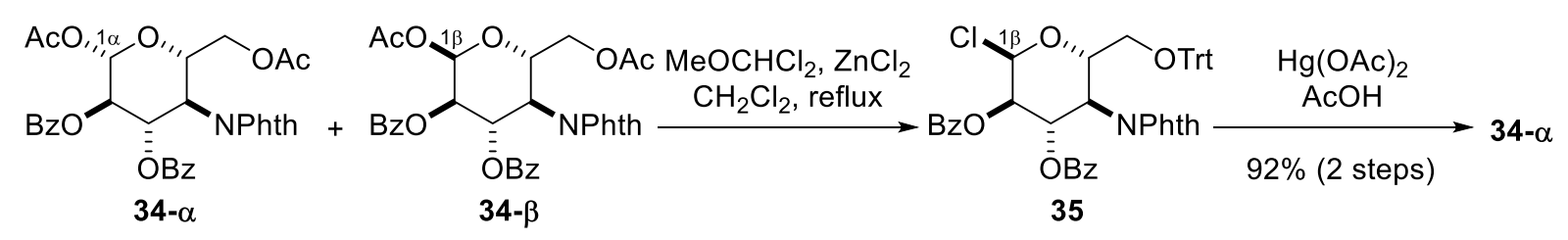

Acetylacetal 34- $\alpha . \quad \mathrm{ZnCl}_{2}(1.0 \mathrm{M}$ THF solution, $12.6 \mathrm{~mL}, 12.6 \mathrm{mmol})$ was added to a solution of a $1: 8.3$ diastereomeric mixture of acetylacetal $34-\alpha$ and $34-\beta(7.60 \mathrm{~g}, 12.6 \mathrm{mmol})$, and 1,1dichloromethyl methyl ether $(17.1 \mathrm{~mL}, 189 \mathrm{mmol})$ in $\mathrm{CH}_{2} \mathrm{Cl}_{2}(840 \mathrm{~mL})$ at $25^{\circ} \mathrm{C}$. The reaction mixture was heated to reflux for $3 \mathrm{~h}$ and then saturated aqueous $\mathrm{NaHCO}_{3}(700 \mathrm{~mL})$ was added 
at $0{ }^{\circ} \mathrm{C}$. The resultant mixture was extracted with $\mathrm{CH}_{2} \mathrm{Cl}_{2}(400 \mathrm{~mL}$ x3). The combined organic layers were washed with brine $(400 \mathrm{~mL})$, dried over $\mathrm{Na}_{2} \mathrm{SO}_{4}$, and concentrated to afford the crude chloroacetal 35 (9.89 g), which was used in the next reaction without further purification.

$\mathrm{Hg}(\mathrm{OAc})_{2}(66.3 \mathrm{mg}, 208 \mu \mathrm{mol})$ was added to a solution of the above crude chloroacetal 35 $(9.89 \mathrm{~g})$ in $\mathrm{AcOH}(126 \mathrm{~mL})$ at $25^{\circ} \mathrm{C}$. After the reaction mixture was stirred at $25^{\circ} \mathrm{C}$ for $8 \mathrm{~h}$, saturated aqueous $\mathrm{NaHCO}_{3}(440 \mathrm{~mL})$ was added at $0{ }^{\circ} \mathrm{C}$. The resultant mixture was extracted with $\mathrm{CH}_{2} \mathrm{Cl}_{2}(440 \mathrm{~mL} \times 3)$. The combined organic layers were washed with brine $(500 \mathrm{~mL})$, dried over $\mathrm{Na}_{2} \mathrm{SO}_{4}$, and concentrated. The residue was purified by flash column chromatography on silica gel $(1500 \mathrm{~g}$, hexane/EtOAc $=10 / 1$ to $2 / 1)$ to afford acetylacetal 34- $\alpha$ $(7.00 \mathrm{~g}, 11.6 \mathrm{mmol})$ in $92 \%$ yield over 2 steps: colorless solid; m.p. $90-91{ }^{\circ} \mathrm{C} ;[\alpha]_{\mathrm{D}}{ }^{24}-14.2(c$ 0.52, $\mathrm{CHCl}_{3}$ ); IR (film) 3066, 2959, 1728, 1381, 1271, 1224, 1100, $1070 \mathrm{~cm}^{-1} ;{ }^{1} \mathrm{H}$ NMR (400 $\left.\mathrm{MHz}, \mathrm{CDCl}_{3}\right): \delta 2.04\left(3 \mathrm{H}, \mathrm{s}, \mathrm{CH}_{3} \mathrm{CO}\right), 2.06\left(3 \mathrm{H}, \mathrm{s}, \mathrm{CH}_{3} \mathrm{CO}\right), 4.14(1 \mathrm{H}, \mathrm{dd}, J=12.8,2.7 \mathrm{~Hz}$, H6a), 4.36 (1H, dd, $J=12.8,3.6 \mathrm{~Hz}, \mathrm{H6b}), 4.74$ (1H, dd, $J=10.1,10.1 \mathrm{~Hz}, \mathrm{H} 4), 4.97$ (1H, ddd, $J=10.1,3.6,2.7 \mathrm{~Hz}, \mathrm{H} 5), 5.66(1 \mathrm{H}, \mathrm{dd}, J=10.1,10.1 \mathrm{~Hz}, \mathrm{H} 2), 6.13(1 \mathrm{H}, \mathrm{d}, J=10.1 \mathrm{~Hz}, \mathrm{H} 1)$, $6.27(1 \mathrm{H}, \mathrm{dd}, J=10.1,10.1 \mathrm{~Hz}, \mathrm{H} 3), 7.24-7.28(2 \mathrm{H}, \mathrm{m}$, aromatic), $7.36(2 \mathrm{H}, \mathrm{t}, J=8.2 \mathrm{~Hz}$, aromatic), $7.42(1 \mathrm{H}, \mathrm{t}, J=7.3 \mathrm{~Hz}$, aromatic), $7.50(1 \mathrm{H}, \mathrm{t}, J=7.3 \mathrm{~Hz}$, aromatic), 7.69-7.74 (5H, m, aromatic), 7.91-7.93 (3H, m, aromatic); $\left.{ }^{13} \mathrm{C} \mathrm{NMR} \mathrm{(100} \mathrm{MHz,} \mathrm{CDCl}_{3}\right): \delta 20.6,20.8,50.5$, 61.9, 70.3, 70.6, 71.3, 92.0, 123.7 (2C), 128.3 (2C), 128.4 (2C), 128.7, 129.7 (2C), 129.8 (2C), 131.0 (2C), 131.3 (2C), 133.4, 133.5, 134.4, 165.0, 165.5, 167.0, 168.1, 169.0, 170.6; HRMS (ESI-TOF) calcd for $\mathrm{C}_{32} \mathrm{H}_{27} \mathrm{NO}_{11} \mathrm{Na}[\mathrm{M}+\mathrm{Na}]^{+}$624.1476, found 624.1453.
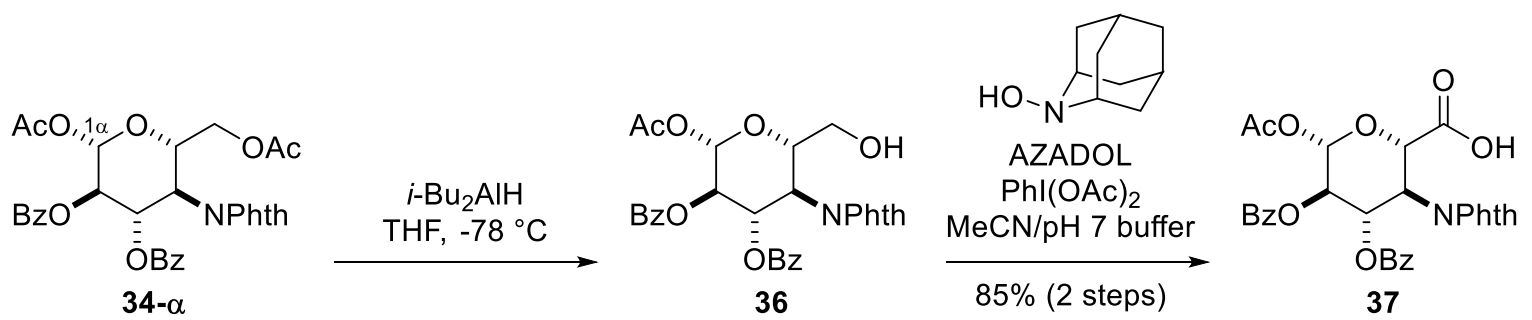

Carboxylic acid 37. $i$-Bu $\mathrm{Bu}_{2} \mathrm{AlH}(1.0 \mathrm{M}$ hexane solution, $26.7 \mathrm{~mL}, 26.7 \mathrm{mmol})$ was slowly added to a solution of acetylacetal $34-\alpha(7.00 \mathrm{~g}, 11.6 \mathrm{mmol})$ in THF $(115 \mathrm{~mL})$ at $-78{ }^{\circ} \mathrm{C}$. After the reaction mixture was stirred at $-78^{\circ} \mathrm{C}$ for $3 \mathrm{~h}$, saturated aqueous Rochelle salt $(530 \mathrm{~mL})$ was added. The resultant mixture was vigorously stirred at $25^{\circ} \mathrm{C}$ for $1 \mathrm{~h}$ and then extracted with EtOAc $(500 \mathrm{~mL} \times 3)$. The combined organic layers were washed with brine $(500 \mathrm{~mL})$, dried over $\mathrm{Na}_{2} \mathrm{SO}_{4}$, and concentrated. The residue was then filtered through a pad of silica gel (300 $\mathrm{g}$, hexane/EtOAc $=1.5 / 1) . \quad$ The filtrate was concentrated to afford the crude alcohol $36(6.63$ 
g), which was used in the next reaction without further purification. To collect the analytical data, 36 was partially purified by flash column chromatography on silica gel (5 g, hexane/EtOAc $=3 / 1$ to $1.5 / 1)$.

36: colorless solid; m.p. $221-223{ }^{\circ} \mathrm{C}$; $[\alpha]_{\mathrm{D}}{ }^{28}-0.465$ (c 0.59, $\mathrm{CHCl}_{3}$ ); IR (film) 3527, 3065, 2955, 1768, 1725, 1382, 1273, $1069 \mathrm{~cm}^{-1}$; ${ }^{1} \mathrm{H}$ NMR (400 MHz, $\left.\mathrm{CDCl}_{3}\right): \delta 2.07$ (3H, s, $\left.\mathrm{CH}_{3} \mathrm{CO}\right), 3.62$ $(1 \mathrm{H}, \mathrm{dd}, J=12.8,3.7 \mathrm{~Hz}, \mathrm{H6a}), 3.85(1 \mathrm{H}, \mathrm{dd}, J=12.8,2.3 \mathrm{~Hz}, \mathrm{H} 6 \mathrm{~b}), 4.79$ (1H, ddd, $J=10.5$, 3.7, $2.3 \mathrm{~Hz}, \mathrm{H} 5), 4.81$ (1H, dd, $J=10.5,10.5 \mathrm{~Hz}, \mathrm{H} 4), 5.64(1 \mathrm{H}, \mathrm{dd}, J=10.5,10.5 \mathrm{~Hz}, \mathrm{H} 2)$, $6.15(1 \mathrm{H}, \mathrm{d}, J=10.5 \mathrm{~Hz}, \mathrm{H} 1), 6.33$ (1H, dd, $J=10.5,10.5 \mathrm{~Hz}, \mathrm{H} 3), 7.25-7.29$ (3H, m, aromatic), $7.37(2 \mathrm{H}, \mathrm{m}$, aromatic), $7.43(1 \mathrm{H}, \mathrm{m}$, aromatic), $7.51(1 \mathrm{H}, \mathrm{m}$, aromatic $), 7.71-7.76(5 \mathrm{H}, \mathrm{m}$, aromatic), 7.91-7.94 (2H, m, aromatic); $\left.{ }^{13} \mathrm{C} \mathrm{NMR} \mathrm{(100} \mathrm{MHz,} \mathrm{CDCl}_{3}\right): \delta 20.8,50.4,61.4,70.7$, 71.4, 72.5, 92.0, 123.7 (2C), 128.3 (2C), 128.4 (2C), 128.7, 129.76 (2C), 129.83 (2C), 131.0 (2C), 131.4 (2C), 133.3, 133.4, 134.4, 165.0, 165.6, 167.3, 167.9, 169.1; HRMS (ESI-TOF) calcd for $\mathrm{C}_{30} \mathrm{H}_{25} \mathrm{NO}_{10} \mathrm{Na}[\mathrm{M}+\mathrm{Na}]^{+}$582.1371, found 582.1345.

2-Hydroxy-2-azaadamantane (AZADOL, $77.3 \mathrm{mg}, 580 \mu \mathrm{mol})$ and $\mathrm{PhI}(\mathrm{OAc})_{2}(13.8 \mathrm{~g}, 16642.9$ mmol) were added to a solution of the above crude alcohol $36(6.63 \mathrm{~g})$ in $\mathrm{CH}_{3} \mathrm{CN}(58.0 \mathrm{~mL})$ and pH 7 phosphate buffer $(58.0 \mathrm{~mL})$ at $25{ }^{\circ} \mathrm{C}$. A reaction mixture was stirred at $25^{\circ} \mathrm{C}$ for 2 $\mathrm{h}$ and then saturated aqueous $\mathrm{Na}_{2} \mathrm{~S}_{2} \mathrm{O}_{3}(420 \mathrm{~mL})$ was added to the mixture at $0{ }^{\circ} \mathrm{C}$. After being vigorously stirred at $0{ }^{\circ} \mathrm{C}$ for $30 \mathrm{~min}$, the resultant mixture was extracted with $\mathrm{CHCl}_{3} / i-\mathrm{PrOH}$ $(5 / 1,500 \mathrm{~mL} \times 3)$. The combined organic layers were washed with brine $(500 \mathrm{~mL})$, dried over $\mathrm{Na}_{2} \mathrm{SO}_{4}$, and concentrated. The residue was purified by flash column chromatography on silica gel $\left(1000 \mathrm{~g}, \mathrm{CHCl}_{3} / \mathrm{MeOH}=100 / 1\right.$ to $\left.5 / 1\right)$ to afford carboxylic acid 37 (5.56 g, 9.98 $\mathrm{mmol})$ in $85 \%$ yield over 2 steps: colorless solid; m.p. $137-138{ }^{\circ} \mathrm{C} ;[\alpha]_{\mathrm{D}}{ }^{28}+9.32(c) 1.13$, $\mathrm{CHCl}_{3}$ ); IR (film) 3487, 3067, 2951, 1727, 1383, 1271, $1083 \mathrm{~cm}^{-1} ;{ }^{1} \mathrm{H} \mathrm{NMR}$ (400 MHz, $\mathrm{CDCl}_{3}$, at $\left.50{ }^{\circ} \mathrm{C}\right): \delta 2.08\left(3 \mathrm{H}, \mathrm{s}, \mathrm{CH}_{3} \mathrm{CO}\right), 4.83(1 \mathrm{H}, \mathrm{dd}, J=10.6,10.6 \mathrm{~Hz}, \mathrm{H} 4), 5.35(1 \mathrm{H}, \mathrm{d}, J=10.6$ $\mathrm{Hz}, \mathrm{H} 5), 5.68(1 \mathrm{H}, \mathrm{t}, J=10.6,10.6 \mathrm{~Hz}, \mathrm{H} 2), 6.16(1 \mathrm{H}, \mathrm{d}, J=10.6 \mathrm{~Hz}, \mathrm{H} 1), 6.35(1 \mathrm{H}, \mathrm{dd}, J=$ 10.6, 10.6 Hz, H3), 7.28 (2H, t, $J=7.3 \mathrm{~Hz}$, aromatic), 7.37 (2H, t, $J=7.8 \mathrm{~Hz}$, aromatic), 7.44 $(1 \mathrm{H}, \mathrm{t}, J=7.8 \mathrm{~Hz}$, aromatic), $7.52(1 \mathrm{H}, \mathrm{t}, J=7.3 \mathrm{~Hz}), 7.69-7.71(2 \mathrm{H}, \mathrm{m}$, aromatic), 7.76 (2H, $\mathrm{d}, J=8.2 \mathrm{~Hz}$, aromatic), $7.81\left(2 \mathrm{H}, \mathrm{m}\right.$, aromatic), $7.92\left(2 \mathrm{H}, \mathrm{d}, J=7.8 \mathrm{~Hz}\right.$, aromatic); ${ }^{13} \mathrm{C} \mathrm{NMR}$ $\left(100 \mathrm{MHz}, \mathrm{CDCl}_{3}\right): \delta 20.8,51.2,69.6,70.2,70.9,91.6,123.9(2 \mathrm{C}), 128.2,128.36(2 \mathrm{C}), 128.47$ (2C), 128.54, 129.8 (2C), 129.9 (2C), 131.2, 133.5, 133.6, 134.4, 164.9, 165.4, 167.4 (2C), 169.0, 169.3 (Two aromatic peaks of the phthalimide were not observed.); HRMS (ESI-TOF) calcd for $\mathrm{C}_{30} \mathrm{H}_{22} \mathrm{NO}_{11}[\mathrm{M}-\mathrm{H}]^{+}$572.1198, found 572.1201. 


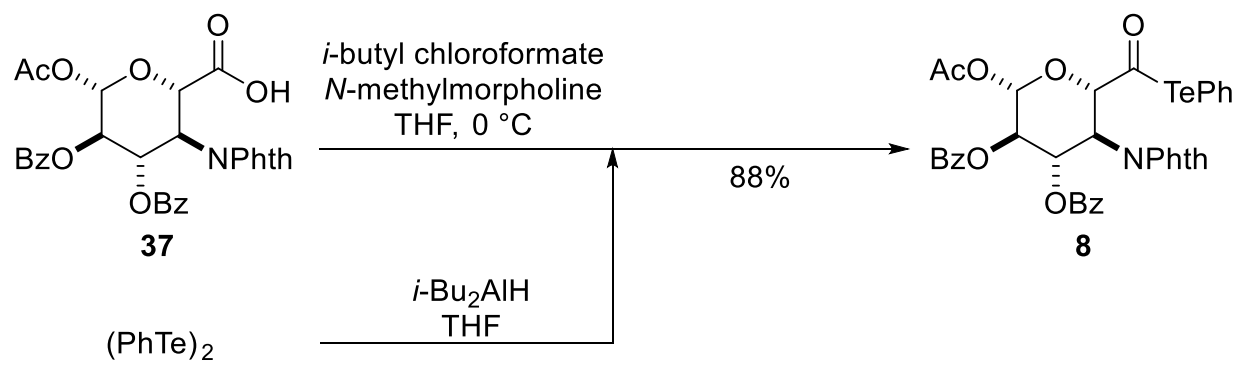

Acyl telluride 8. $\quad N$-methylmorpholine $(76.0 \mu \mathrm{L}, 651 \mu \mathrm{mol})$ and isobutyl chloroformate $(82.4$ $\mu \mathrm{L}, 651 \mu \mathrm{mol})$ were added to a solution of carboxylic acid 37 (249 mg, $434 \mu \mathrm{mol})$ in THF (2.17 $\mathrm{mL})$ at $0{ }^{\circ} \mathrm{C}$. The reaction mixture was stirred at $0{ }^{\circ} \mathrm{C}$ for $30 \mathrm{~min}$. $i$ - $\mathrm{Bu}_{2} \mathrm{AlH}(1.0 \mathrm{M}$ hexane solution, $868 \mu \mathrm{L}, 868 \mu \mathrm{mol})$ was added to a solution of (PhTe) $2(178 \mathrm{mg}, 434 \mu \mathrm{mol})$ in THF $(2.17 \mathrm{~mL})$ at $25{ }^{\circ} \mathrm{C}$. After being stirred at $25{ }^{\circ} \mathrm{C}$ for $5 \mathrm{~min}$, the latter reaction mixture was added dropwise to the former one. The resultant mixture was stirred at $25^{\circ} \mathrm{C}$ for $30 \mathrm{~min}$ and then was filtered through a pad of silica gel ( $5 \mathrm{~g}$, EtOAc). The filtrate was concentrated and the residue was purified by flash column chromatography on silica gel (Fuji Silysia, $30 \mathrm{~g}$, hexane/EtOAc $=10 / 1$ to $2.5 / 1)$ to afford acyl telluride $8(291 \mathrm{mg}, 382 \mu \mathrm{mol})$ in $88 \%$ yield: pale yellow solid; m.p. $109-111^{\circ} \mathrm{C}$; $[\alpha]_{\mathrm{D}}{ }^{19}+1.45\left(c 0.34, \mathrm{CHCl}_{3}\right)$; IR (film) 3064, 2970, 1773, 1728 , 1383, 1272, 1069, $713 \mathrm{~cm}^{-1}$; ${ }^{1} \mathrm{H}$ NMR $\left(500 \mathrm{MHz}, \mathrm{CDCl}_{3}\right) \delta 2.18\left(3 \mathrm{H}, \mathrm{s}, \mathrm{CH} \mathrm{COO}_{3}\right), 477(1 \mathrm{H}, \mathrm{dd}$, $J=10.9,10.9 \mathrm{~Hz}, \mathrm{H} 4), 5.05(1 \mathrm{H}, \mathrm{d}, J=10.9 \mathrm{~Hz}, \mathrm{H} 5), 5.72(1 \mathrm{H}, \mathrm{dd}, J=10.9,8.1 \mathrm{~Hz}, \mathrm{H} 2), 6.27$ $(1 \mathrm{H}, \mathrm{d}, J=8.1 \mathrm{~Hz}, \mathrm{H} 1), 6.33$ (1H, dd, $J=10.9,10.9 \mathrm{~Hz}, \mathrm{H} 3), 7.25-7.34$ (5H, m, aromatic), 7.39 $(2 \mathrm{H}, \mathrm{t}, J=8.0 \mathrm{~Hz}$, aromatic), $7.45(1 \mathrm{H}, \mathrm{t}, J=7.5 \mathrm{~Hz}$, aromatic), $7.53(1 \mathrm{H}, \mathrm{t}, J=6.3 \mathrm{~Hz}$, aromatic), 7.63-7.64 (4H, m, aromatic), 7.76-7.79 (4H, m, aromatic), $7.96(2 \mathrm{H}, \mathrm{d}, J=8.0 \mathrm{~Hz}$, aromatic) ${ }^{13} \mathrm{C} \mathrm{NMR}\left(125 \mathrm{MHz}, \mathrm{CDCl}_{3}\right): \delta 20.8,50.1,70.0,70.8,79.0,91.6,113.2,123.7(2 \mathrm{C}), 128.2$, 128.3 (2C), 128.48 (2C), 128.50, 128.9, 129.2, 129.5 (2C), 129.7, 129.8 (2C), 129.9 (2C), 131.2, 131.3, 133.5, 133.6, 134.2, 137.5, 140.3 (2C), 164.9, 165.4, 168.8, 204.1; HRMS (ESI-TOF) calcd for $\mathrm{C}_{36} \mathrm{H}_{27} \mathrm{NO}_{10} \mathrm{TeNa}[\mathrm{M}+\mathrm{Na}]^{+}$786.0589, found 786.0561 . 


\section{General procedure A: Decarbonylative radical addition of $8 b$}

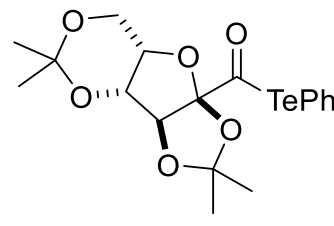

19

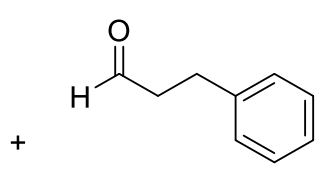

20

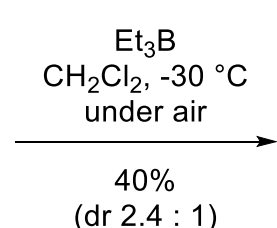

$(\mathrm{dr} 2.4: 1)$

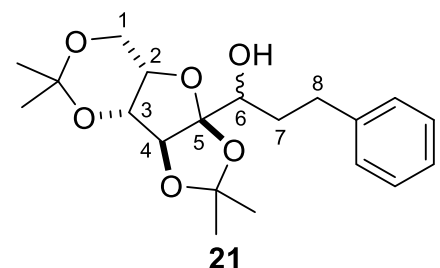

21

Alcohol 21. $\mathrm{Et}_{3} \mathrm{~B}(1.0 \mathrm{M}$ in hexane solution, $450 \mu \mathrm{L}, 450 \mu \mathrm{mol})$ was added to a solution of acyl telluride $19^{\mathrm{S} 5}(41.9 \mathrm{mg}, 90.7 \mu \mathrm{mol})$ and aldehyde 20 (36.5 mg, $\left.272 \mu \mathrm{mol}\right)$ in $\mathrm{CH}_{2} \mathrm{Cl}_{2}(910$ $\mu \mathrm{L}$ ) at $-30{ }^{\circ} \mathrm{C}$. After being stirred at $-30{ }^{\circ} \mathrm{C}$ for $30 \mathrm{~min}$ under air, the reaction mixture was concentrated and azeotroped with $\mathrm{MeOH}(2 \mathrm{~mL} \times 2)$. The residue was dissolved in $\mathrm{CH}_{2} \mathrm{Cl}_{2}(1$ $\mathrm{mL})$ and filtered through a pad of amine-modified silica gel (10 g, EtOAc). The filtrate was concentrated and the residue was purified by flash chromatography on silica gel $(10 \mathrm{~g}$, hexane/EtOAc $=25 / 1$ to 3/1) to afford a $2.4: 1$ diastereomeric mixture of alcohol 21 (13.2 $\mathrm{mg}$, $36.2 \mu \mathrm{mol}$ ) in 40\% yield: colorless oil; IR (film) 3504, 3026, 2990, 2935, 2867, 1454, 1378, 1198, 1122, $1080 \mathrm{~cm}^{-1} ;{ }^{1} \mathrm{H}$ NMR (400 MHz, $\left.\mathrm{CDCl}_{3}\right): \delta 1.27\left(3.00 \mathrm{H}, \mathrm{s}, \mathrm{CH}_{3}\right.$ of acetonide), 1.33 $\left(0.88 \mathrm{H}, \mathrm{s}, \mathrm{CH}_{3}\right.$ of acetonide), $1.39\left(2.12 \mathrm{H}, \mathrm{s}, \mathrm{CH}_{3}\right.$ of acetonide), $1.41\left(2.12 \mathrm{H}, \mathrm{s}, \mathrm{CH}_{3}\right.$ of acetonide), $1.44\left(0.88 \mathrm{H}, \mathrm{s}, \mathrm{CH}_{3}\right.$ of acetonide), $1.48\left(0.88 \mathrm{H}, \mathrm{s}, \mathrm{CH}_{3}\right.$ of acetonide), $1.50(2.12 \mathrm{H}$, s, $\mathrm{CH}_{3}$ of acetonide), $1.77(0.71 \mathrm{H}, \mathrm{m}, \mathrm{H} 7), 1.93(0.29 \mathrm{H}, \mathrm{m}, \mathrm{H} 7), 2.07(0.71 \mathrm{H}, \mathrm{d}, J=8.2 \mathrm{~Hz}$, $\mathrm{OH}), 2.13(0.29 \mathrm{H}, \mathrm{m}, \mathrm{H} 7), 2.26(0.71 \mathrm{H}, \mathrm{m} . \mathrm{H} 7), 2.56(0.29 \mathrm{H}, \mathrm{d}, J=7.3 \mathrm{~Hz}, \mathrm{OH}), 2.74(1.00 \mathrm{H}$, m, H8), $2.97(1.00 \mathrm{H}, \mathrm{m}, \mathrm{H} 8), 3.71(0.29 \mathrm{H}, \mathrm{m}, \mathrm{H} 6), 3.82(0.71 \mathrm{H}, \mathrm{m}, \mathrm{H} 6), 4.03-4.11(3.00 \mathrm{H}, \mathrm{m}$, H1, H2), $4.28(0.29 \mathrm{H}, \mathrm{d}, J=2.3 \mathrm{~Hz}, \mathrm{H} 3), 4.29(0.71 \mathrm{H}, \mathrm{d}, J=2.3 \mathrm{~Hz}, \mathrm{H} 3), 4.49(0.71 \mathrm{H}, \mathrm{s}, \mathrm{H} 4)$, $4.50(0.29 \mathrm{H}, \mathrm{s}, \mathrm{H} 4), 7.17(1.00 \mathrm{H}, \mathrm{m}$, aromatic $), 7.24-7.29(4.00 \mathrm{H}, \mathrm{m}$, aromatic $) ;{ }^{13} \mathrm{C}$ NMR (100 $\left.\mathrm{MHz}, \mathrm{CDCl}_{3}\right): \delta 18.5$ (0.29C), 18.6 (0.71C), 26.4 (0.29C), 27.1 (0.71C), 27.3 (0.29C), 27.8 (0.71C), 28.7 (0.71C), 28.8 (0.29C), 31.98 (0.71C), 32.01 (0.29C), 32.8 (0.29C), 33.0 (0.71C), 60.2 (0.29C), 60.4 (0.71C), 71.2 (0.71C), 71.9 (0.29C), 72.3 (0.71C), 72.7 (0.29C), 73.1 (0.71C), 73.3 (0.29C), 84.6 (0.71C), 86.0 (0.29C), 97.3 (0.71C), 97.6 (0.29C), 111.6 (0.29C), 112.3 (0.71C), 115.5 (0.29C), 116.3 (0.71C), 125.55 (0.29C), 125.64 (0.71C), 128.1 (0.58C), 128.2 (1.42C), 128.51 (1.42C), 128.55 (0.58C), 141.9 (0.71C), 142.1 (0.29C); HRMS (ESITOF) calcd for $\mathrm{C}_{20} \mathrm{H}_{28} \mathrm{O}_{6} \mathrm{Na}[\mathrm{M}+\mathrm{Na}]^{+} 387.1778$, found 387.1793 . 


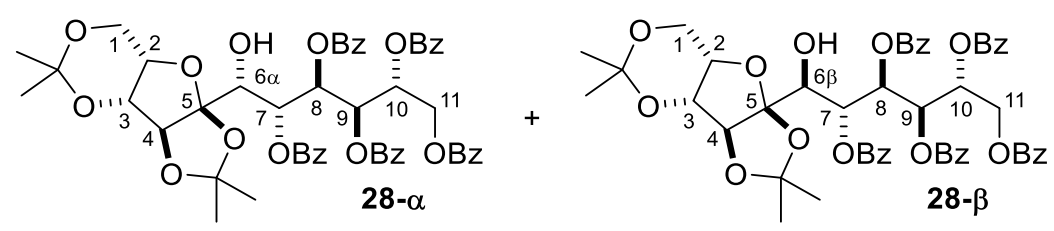

Alcohols 28- $\boldsymbol{\alpha}$ and 28- $\boldsymbol{\beta}$. According to the general procedure A, alcohols $\mathbf{2 8 - \alpha}$ (37.0 $\mathrm{mg}, 39.7$ $\mu \mathrm{mol})$ and 28- $\boldsymbol{\beta}$ (41.9 mg, $45.0 \mu \mathrm{mol})$ were synthesized in $17 \%$ and $19 \%$ yields, respectively, from acyl telluride 19 (108 mg, $234 \mu \mathrm{mol}$ ) and aldehyde 7a (492 mg, $702 \mu \mathrm{mol}$ ) by using $\mathrm{Et}_{3} \mathrm{~B}$ (1.0 $\mathrm{M}$ in hexane, $1.17 \mathrm{~mL}, 1.17 \mathrm{mmol})$ in $\mathrm{CH}_{2} \mathrm{Cl}_{2}(2.34 \mathrm{~mL})$. The residue was purified by flash column chromatography on amine-modified silica gel (5 g, EtOAc) and silica gel (10 g, hexane $/$ EtOAc $=10 / 1$ to $2 / 1)$, and then by PTLC $(10 \mathrm{~cm} \times 20 \mathrm{~cm}, 2$ plates, hexane/ EtOAc $=$ 4/3) and PTLC $(10 \mathrm{~cm} \times 20 \mathrm{~cm}, 2$ plates, toluene/EtOAc $=4 / 1)$. The structure of 28- $\alpha$ was determined by the X-ray crystallographic analysis (Page S31).

28- $\alpha$ : colorless plate crystal (recrystallization from hexane/EtOAc); m.p. $87-89{ }^{\circ} \mathrm{C} ;[\alpha]_{\mathrm{D}}{ }^{25}$ +28.6 (c 0.78, $\mathrm{CHCl}_{3}$ ); IR (film) 3514, 3065, 2989, 2926, 1727, 1451,1379, 1260, $1096 \mathrm{~cm}^{-1}$; ${ }^{1} \mathrm{H}$ NMR (400 MHz, $\left.\mathrm{CDCl}_{3}\right): \delta 0.89\left(3 \mathrm{H}, \mathrm{s}, \mathrm{CH}_{3}\right.$ of acetonide), 1.17 (3H, s, $\mathrm{CH}_{3}$ of acetonide), $1.18\left(3 \mathrm{H}, \mathrm{s}, \mathrm{CH}_{3}\right.$ of acetonide), $1.27\left(3 \mathrm{H}, \mathrm{s}, \mathrm{CH}_{3}\right.$ of acetonide), $2.85(1 \mathrm{H}, \mathrm{d}, J=11.0 \mathrm{~Hz}, \mathrm{OH})$, $3.90(1 \mathrm{H}, \mathrm{dd}, J=13.7,2.8 \mathrm{~Hz}, \mathrm{H} 1 \mathrm{a}), 4.00(1 \mathrm{H}, \mathrm{d}, J=13.7 \mathrm{~Hz}, \mathrm{H} 1 \mathrm{~b}), 4.08$ (1H, dd, $J=2.8,2.3$ Hz, H2), $4.13(1 \mathrm{H}, \mathrm{dd}, J=11.0,2.7 \mathrm{~Hz}, \mathrm{H} 6), 4.21(1 \mathrm{H}, \mathrm{d}, J=2.3 \mathrm{~Hz}, \mathrm{H} 3), 4.52(1 \mathrm{H}, \mathrm{s}, \mathrm{H} 4)$, $4.53(1 \mathrm{H}, \mathrm{dd}, J=12.4,6.9 \mathrm{~Hz}, \mathrm{H} 11 \mathrm{a}), 4.76(1 \mathrm{H}, \mathrm{dd}, J=12.4,3.6 \mathrm{~Hz}, \mathrm{H} 11 \mathrm{~b}), 5.86(1 \mathrm{H}, \mathrm{ddd}, \mathrm{J}$ $=7.4,6.9,3.6 \mathrm{~Hz}, \mathrm{H} 10), 6.11(1 \mathrm{H}, \mathrm{dd}, J=7.8,2.7 \mathrm{~Hz}, \mathrm{H} 7), 6.15(1 \mathrm{H}, \mathrm{dd}, J=7.4,1.8 \mathrm{~Hz}, \mathrm{H} 9)$, $6.26(1 \mathrm{H}, \mathrm{dd}, J=7.8,1.8 \mathrm{~Hz}, \mathrm{H} 8), 7.23-7.32(5 \mathrm{H}, \mathrm{m}$, aromatic), 7.36-7.55 (10H, m, aromatic), 7.81-7.88 (6H, m, aromatic), 8.05-8.09 (4H, m, aromatic); Detectable signals of ${ }^{13} \mathrm{C}$ NMR (125 $\left.\mathrm{MHz}_{,} \mathrm{CDCl}_{3}\right): \delta 18.6,26.6,27.4,28.1,60.2,63.2,68.9,69.1,69.6,69.9,70.0,72.5,73.4,85.3$, $97.2,112.8,114.5,128.0,128.1,128.4,129.2,129.5,129.65,129.67,129.71,130.0,130.1$, 130.2, 132.7, 132.8, 132.9, 133.1, 133.3, 165.3, 165.4, 165.5, 166.0; HRMS (ESI-TOF) calcd for $\mathrm{C}_{52} \mathrm{H}_{50} \mathrm{O}_{16} \mathrm{Na}[\mathrm{M}+\mathrm{Na}]^{+}$953.2991, found 953.2978.

28- $\beta$ : colorless solid; m.p. $64-67^{\circ} \mathrm{C}$; $[\alpha]_{\mathrm{D}}{ }^{25}+38.2\left(c 0.50, \mathrm{CHCl}_{3}\right)$; IR (film) 3504, 3437, 3064 , 2990, 2935, 1726, 1451, 1263, 1109, $710 \mathrm{~cm}^{-1} ;{ }^{1} \mathrm{H} \mathrm{NMR}\left(400 \mathrm{MHz}, \mathrm{CDCl}_{3}\right): \delta 1.18\left(3 \mathrm{H}, \mathrm{s}, \mathrm{CH}_{3}\right.$ of acetonide), 1.24 (3H, s, $\mathrm{CH}_{3}$ of acetonide), 1.42 (3H, s, $\mathrm{CH}_{3}$ of acetonide), 1.44 (3H, s, $\mathrm{CH}_{3}$ of acetonide), $3.53(1 \mathrm{H}, \mathrm{d}, J=7.3 \mathrm{~Hz}, \mathrm{OH}), 4.01-4.04(2 \mathrm{H}, \mathrm{m}, \mathrm{H} 1 \mathrm{a}, \mathrm{H} 2), 4.10(1 \mathrm{H}, \mathrm{d}, J=12.3$ Hz, H1b), 4.28 (1H, d, $J=1.8$ Hz, H3), 4.53-4.61 (3H, m, H4, H6, H11a), 4.87 (1H, dd, $J=$ 12.4, 3.2 Hz, H11b), 5.83 (1H, ddd, $J=7.3,6.8,3.2 \mathrm{~Hz} \mathrm{H10),} 6.13(1 \mathrm{H}, \mathrm{dd}, J=8.2,4.1 \mathrm{~Hz}$, H7), $6.28(1 \mathrm{H}, \mathrm{dd}, J=4.1,2.3 \mathrm{~Hz}, \mathrm{H} 8), 6.56(1 \mathrm{H}, \mathrm{dd}, J=6.8,2.3 \mathrm{~Hz}, \mathrm{H} 9), 7.01(2 \mathrm{H}, \mathrm{t}, J=7.3$ $\mathrm{Hz}$, aromatic), 7.28-7.32 (4H, m, aromatic), 7.34-7.38 (5H, m, aromatic), 7.46-7.53 (4H, m, 
aromatic), 7.66-7.68 (2H, m, aromatic), 7.96-8.00 (8H, m, aromatic); Detectable signals of ${ }^{13} \mathrm{C}$ NMR (100 MHz, $\left.\mathrm{CDCl}_{3}\right): \delta 18.6,26.1,26.7,28.7,59.9,62.8,62.9,69.3,69.81,69.83,69.85$, $70.21,70.25,71.33,71.36,71.39,72.1,72.3,72.4,73.18,73.21,87.11,87.14,97.9,112.3$, 113.9 , 127.6, 128.2, 128.3, 129.56, 129.57, 129.61, 129.68, 129.80, 129.86, 129.92, 132.3, 132.9, 133.0, 133.1, 165.2, 165.3, 165.4, 166.1; HRMS (ESI-TOF) calcd for $\mathrm{C}_{52} \mathrm{H}_{50} \mathrm{O}_{16} \mathrm{Na}$ $[\mathrm{M}+\mathrm{Na}]^{+}$953.2991, found 953.2972.
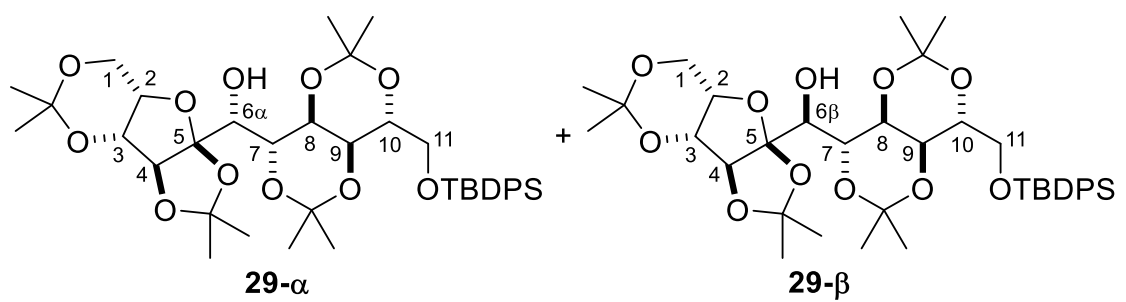

Alcohols 29- $\alpha$ and 29- $\beta$. According to the general procedure A, alcohols 29- $\alpha$ (30.4 mg, 41.7 $\mu \mathrm{mol})$ and $29-\beta$ (14.0 $\mathrm{mg}, 19.2 \mu \mathrm{mol})$ were synthesized in $45 \%$ and $21 \%$ yields, respectively, from acyl telluride 19 (42.4 mg, $91.7 \mu \mathrm{mol})$ and aldehyde $7 \mathbf{b}$ (137 mg, $275 \mu \mathrm{mol})$ by using $\mathrm{Et}_{3} \mathrm{~B}$ (1.0 $\mathrm{M}$ in hexane, $459 \mu \mathrm{L}, 459 \mu \mathrm{mol})$ in $\mathrm{CH}_{2} \mathrm{Cl}_{2}(920 \mu \mathrm{L})$. The residue was purified by flash column chromatography on amine-modified silica gel (2 g, EtOAc) and silica gel (2 g, hexane/EtOAc $=25 / 1$ to 2/1), and then by PTLC $(10 \mathrm{~cm} \times 20 \mathrm{~cm}, 2$ plates, hexane/ EtOAc $=$ 1.8/1). The structure of 29- $\alpha$ was determined by the NMR analysis (Page S33).

29- $\alpha$ : colorless oil; $[\alpha]_{\mathrm{D}}{ }^{25}+0.463$ (c 1.31, $\mathrm{CHCl}_{3}$ ); IR (film) 3569, 3050, 2989, 2934, 2860, 1459, 1429, 1378, 1220, 1111, $1081 \mathrm{~cm}^{-1} ;{ }^{1} \mathrm{H}$ NMR (400 MHz, $\left.\mathrm{C}_{6} \mathrm{D}_{6}\right): \delta 1.03\left(3 \mathrm{H}, \mathrm{s}, \mathrm{CH}_{3}\right.$ of acetonide), 1.18 (9H, s, $\mathrm{C}\left(\mathrm{CH}_{3}\right)_{3}$ of TBDPS), $1.27\left(3 \mathrm{H}, \mathrm{s}, \mathrm{CH}_{3}\right.$ of acetonide), 1.31 (3H, s, $\mathrm{CH}_{3}$ of acetonide), 1.35 (3H, s, $\mathrm{CH}_{3}$ of acetonide), 1.37 (3H, s, $\mathrm{CH}_{3}$ of acetonide), $1.43\left(3 \mathrm{H}, \mathrm{s}, \mathrm{CH}_{3}\right.$ of acetonide), $1.46\left(3 \mathrm{H}, \mathrm{s}, \mathrm{CH}_{3}\right.$ of acetonide), $1.62\left(3 \mathrm{H}, \mathrm{s}, \mathrm{CH}_{3}\right.$ of acetonide), $2.75(1 \mathrm{H}, \mathrm{d}, J=$ $9.2 \mathrm{~Hz}, \mathrm{OH}), 3.41(1 \mathrm{H}, \mathrm{dd}, J=13.3,2.3 \mathrm{~Hz}, \mathrm{H} 1 \mathrm{a}), 3.77(1 \mathrm{H}, \mathrm{m}, \mathrm{H} 2), 3.83(1 \mathrm{H}, \mathrm{d}, J=13.3 \mathrm{~Hz}$, H1b), 3.95-3.96 (2H, m, H11), 4.06-4.15 (3H, m, H3, H9, H10), 4.36 (1H, dd, $J=12.4,0.76$ Hz, H6), 4.53 (1H, dd, $J=9.2,4.6 \mathrm{~Hz}, \mathrm{H} 8), 4.75(1 \mathrm{H}, \mathrm{s}, \mathrm{H} 4), 4.36(1 \mathrm{H}, \mathrm{dd}, J=9.2,0.76 \mathrm{~Hz}$, H7), 7.20-7.24 (1H, m, 6H, aromatic), 7.83-7.88 (1H, m, 4H, aromatic); ${ }^{13} \mathrm{C}$ NMR (125 MHz, $\left.\mathrm{C}_{6} \mathrm{D}_{6}\right): \delta 18.8,19.6,23.9,24.1,25.0,25.3,26.7,27.0$ (3C), 28.0, 28.8, 60.1, 64.8, 67.5, 68.5, 68.6, 69.9, 71.6, 73.0, 73.3, 86.3, 97.2, 100.8, 101.4, 113.0, 115.5, 128.5 (2C), 129.82 (2C), 129.84 (2C), 134.17, 134.19, 136.1 (2C), 136.2 (2C); HRMS (ESI-TOF) calcd for $\mathrm{C}_{39} \mathrm{H}_{56} \mathrm{O}_{11} \mathrm{SiNa}[\mathrm{M}+\mathrm{Na}]^{+}$751.3484, found 751.3470.

29- $\beta$ : colorless oil; $[\alpha]_{\mathrm{D}}{ }^{25}-9.87$ (c 0.91, $\mathrm{CHCl}_{3}$ ); IR (film) 3541, 2989, 2934, 2860, 1459, 1429 , 1378, 1220, 1110, 1084, 1037, $705 \mathrm{~cm}^{-1}$; ${ }^{1} \mathrm{H}$ NMR (400 MHz, $\left.\mathrm{C}_{6} \mathrm{D}_{6}\right): \delta 0.97\left(3 \mathrm{H}, \mathrm{s}, \mathrm{CH}_{3}\right.$ of 
acetonide), $1.20\left(9 \mathrm{H}, \mathrm{s}, \mathrm{C}\left(\mathrm{CH}_{3}\right)_{3}\right.$ of TBDPS $), 1.20\left(3 \mathrm{H}, \mathrm{s}, \mathrm{CH}_{3}\right.$ of acetonide), $1.28\left(3 \mathrm{H}, \mathrm{s}, \mathrm{CH}_{3}\right.$ of acetonide), 1.38 (3H, s, $\mathrm{CH}_{3}$ of acetonide), 1.41 (3H, s, $\mathrm{CH}_{3}$ of acetonide), 1.45 (3H, s, $\mathrm{CH}_{3}$ of acetonide), $1.53\left(3 \mathrm{H}, \mathrm{s}, \mathrm{CH}_{3}\right.$ of acetonide), $1.54\left(3 \mathrm{H}, \mathrm{s}, \mathrm{CH}_{3}\right.$ of acetonide), $2.97(1 \mathrm{H}, \mathrm{d}, J=$ $9.2 \mathrm{~Hz}, \mathrm{OH}), 3.45(1 \mathrm{H}, \mathrm{dd}, J=13.7,2.3 \mathrm{~Hz}, \mathrm{H} 1 \mathrm{a}), 3.69$ (1H, m, H2), 3.85 (1H, d, $J=13.7 \mathrm{~Hz}$, H1b), 3.96-4.01 (3H, m, H3, H11), 4.05 (1H, m, H9), 4.14-4.18 (2H, m, H6, H10), 4.59-4.60 $(2 \mathrm{H}, \mathrm{H} 7, \mathrm{H} 8), 4.78(1 \mathrm{H}, \mathrm{s}, \mathrm{H} 4), 7.21-7.24\left(6 \mathrm{H}, \mathrm{m}\right.$, aromatic), 7.85-7.91 (4H, m, aromatic); ${ }^{13} \mathrm{C}$ NMR (125 MHz, $\left.\mathrm{C}_{6} \mathrm{D}_{6}\right): \delta 18.5,19.6,24.2,24.3,24.6,24.9,26.7,27.0$ (3C), 27.5, 29.1, 60.1, 65.1, 68.9, 69.0, 70.1, 72.0, 72.2, 74.0, 75.5, 87.3, 97.4, 100.7, 100.9, 111.5, 115.7, 127.9 (4C), 129.81, 129.83, 134.19, 134.25, 136.1 (2C), 136.2 (2C); HRMS (ESI-TOF) calcd for $\mathrm{C}_{39} \mathrm{H}_{56} \mathrm{O}_{11} \mathrm{SiNa}[\mathrm{M}+\mathrm{Na}]^{+}$751.3484, found 751.3478 .

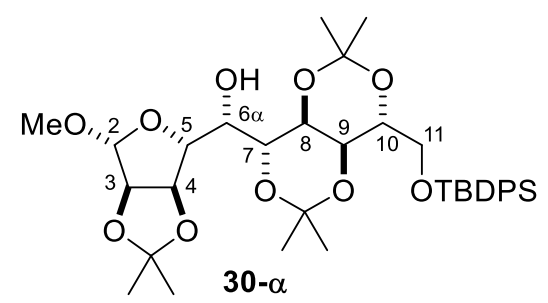

Alcohol 30- $\alpha$. According to the general procedure A, alcohol 30- $\alpha(51.5 \mathrm{mg}, 76.5 \mu \mathrm{mol})$ was synthesized in 77\% yield from acyl telluride $27(40.5 \mathrm{mg}, 99.8 \mu \mathrm{mol})$ and aldehyde $\mathbf{7 b}$ (149 mg, $299 \mu \mathrm{mol})$ by using $\mathrm{Et}_{3} \mathrm{~B}(1.0 \mathrm{M}$ in hexane, $499 \mu \mathrm{L}, 499 \mu \mathrm{mol})$ in $\mathrm{CH}_{2} \mathrm{Cl}_{2}(998 \mu \mathrm{L})$. The residue was purified by flash column chromatography on amine-modified silica gel (2 g, hexane/EtOAc $=1 / 2)$ and silica gel $(5 \mathrm{~g}$, hexane/EtOAc $=25 / 1$ to $2 / 1)$. The structure of $\mathbf{3 0}-\boldsymbol{\alpha}$ was determined by the modified Mosher method (Page S33): colorless oil; $[\alpha]_{\mathrm{D}}{ }^{27}-22.6$ (c 1.03, $\mathrm{CHCl}_{3}$ ); IR (film) 3474, 2987, 2934, 2858, 1379, 1218,1107, $704 \mathrm{~cm}^{-1} ;{ }^{1} \mathrm{H}$ NMR (400 MHz, $\left.\mathrm{C}_{6} \mathrm{D}_{6}\right): \delta 1.11\left(3 \mathrm{H}, \mathrm{s}, \mathrm{CH}_{3}\right.$ of acetonide), $1.16\left(3 \mathrm{H}, \mathrm{s}, \mathrm{CH}_{3}\right.$ of acetonide), $1.19\left(9 \mathrm{H}, \mathrm{s}, \mathrm{C}\left(\mathrm{CH}_{3}\right)_{3}\right.$ of TBDPS), $1.30\left(3 \mathrm{H}, \mathrm{s}, \mathrm{CH}_{3}\right.$ of acetonide), $1.32\left(3 \mathrm{H}, \mathrm{s}, \mathrm{CH}_{3}\right.$ of acetonide), $1.38\left(3 \mathrm{H}, \mathrm{s}, \mathrm{CH}_{3}\right.$ of acetonide), $1.50\left(3 \mathrm{H}, \mathrm{s}, \mathrm{CH}_{3}\right.$ of acetonide), $2.34(1 \mathrm{H}, \mathrm{d}, J=10.0 \mathrm{~Hz}, \mathrm{OH}), 3.16\left(3 \mathrm{H}, \mathrm{s}, \mathrm{OCH}_{3}\right)$, 3.82 (1H, dd, $J=8.7,4.6$ Hz, H9), 3.86-3.97 (3H, m, H6, H11), 4.05 (1H, m, H10), 4.22 (1H, d, $J=9.2 \mathrm{~Hz}, \mathrm{H} 7), 4.31(1 \mathrm{H}, \mathrm{dd}, J=9.2,4.6 \mathrm{~Hz}, \mathrm{H} 8), 4.47(1 \mathrm{H}, \mathrm{d}, J=9.2 \mathrm{~Hz}, \mathrm{H} 5), 4.64(1 \mathrm{H}$, $\mathrm{d}, J=6.4 \mathrm{~Hz}, \mathrm{H} 3), 5.08$ (1H, s, H2), $5.19(1 \mathrm{H}, \mathrm{d}, J=6.4 \mathrm{~Hz}, \mathrm{H} 4), 7.22-7.25$ (6H, m, aromatic), 7.82-7.88 (4H, m, aromatic); ${ }^{13} \mathrm{C}$ NMR (100 MHz, $\left.\mathrm{C}_{6} \mathrm{D}_{6}\right): \delta$ 19.6, 23.6, 24.0, 24.7, 24.9 (2C), 26.6, 26.9 (3C), 55.1, 64.8, 67.6, 68.5, 68.6, 70.7, 71.5, 82.6, 85.8, 87.3, 100.7, 101.3, 110.1, 112.0, 128.3 (4C), 129.89, 129.93, 134.0, 134.1, 136.1 (2C), 136.2 (2C); HRMS (ESI-TOF) calcd for $\mathrm{C}_{36} \mathrm{H}_{52} \mathrm{O}_{10} \mathrm{SiNa}[\mathrm{M}+\mathrm{Na}]^{+}$695.3222, found 695.3240 . 

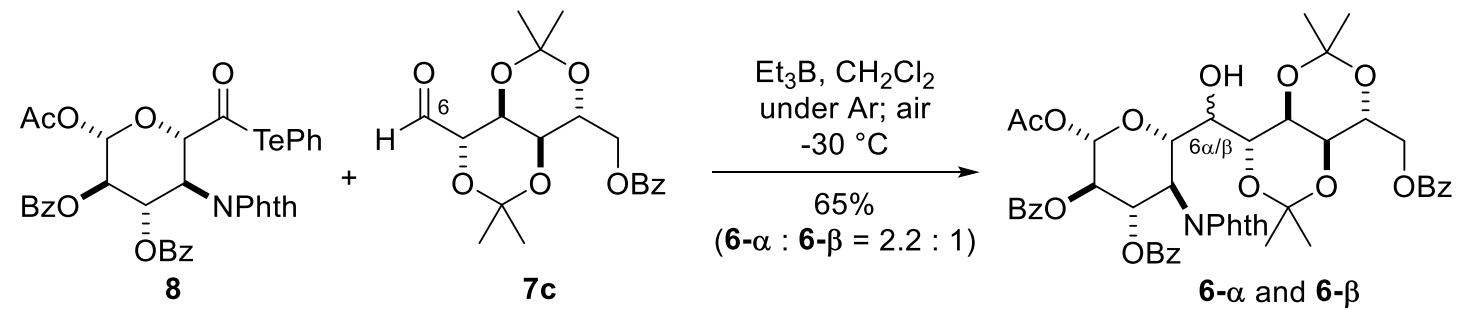

Alcohols 6- $\boldsymbol{\alpha}$ and 6- $\beta$. $\mathrm{Et}_{3} \mathrm{~B}(1.0 \mathrm{M}$ in hexane solution, $880 \mu \mathrm{L}, 880 \mu \mathrm{mol})$ was added to a solution of acyl telluride 8 (134 mg, $176 \mu \mathrm{mol})$ and aldehyde $7 \mathbf{c}(192 \mathrm{mg}, 527 \mu \mathrm{mol})$ in $\mathrm{CH}_{2} \mathrm{Cl}_{2}$ $(1.76 \mathrm{~mL})$ at $-30{ }^{\circ} \mathrm{C}$ under argon atmosphere. A disposable needle was inserted through the rubber septa cap of the reaction flask to introduce air. After being stirred at $-30{ }^{\circ} \mathrm{C}$ for $13 \mathrm{~h}$, the reaction mixture was concentrated. The crude mixture was dissolved in $\mathrm{CH}_{2} \mathrm{Cl}_{2}(880 \mu \mathrm{L})$ and $\mathrm{MeOH}(880 \mu \mathrm{L})$, and then $\mathrm{TrisNHNH}_{2}(236 \mathrm{mg}, 791 \mu \mathrm{mol})$ was added to the reaction mixture at $25{ }^{\circ} \mathrm{C}$ to convert the recovered aldehyde $7 \mathbf{c}$ to the corresponding hydrazide. After being stirred at $25^{\circ} \mathrm{C}$ for $1 \mathrm{~h}$, the reaction mixture was concentrated. The residue was purified by flash chromatography on silica gel (30 g, hexane/EtOAc $=3 / 1$ to $2 / 1)$ and GPC to afford a 2.2 : 1 diastereomeric mixture of alcohol $\mathbf{6}-\boldsymbol{\alpha}$ and $\mathbf{6 - \beta}(103 \mathrm{mg}, 115 \mu \mathrm{mol})$ in $65 \%$ yield. To collect the analytical data, $\mathbf{6}-\boldsymbol{\alpha}$ and $\mathbf{6}-\boldsymbol{\beta}$ were partially purified by PTLC $(10 \mathrm{~cm}$ x $20 \mathrm{~cm}, 1$ plate, toluene/EtOAc $=8 / 1)$. The structures of $\mathbf{6}-\boldsymbol{\alpha}$ and $\mathbf{6}-\boldsymbol{\beta}$ were determined by the CP3 analysis (Page S38).

6- $\alpha$ : colorless solid; m.p. $103-105^{\circ} \mathrm{C} ;[\alpha]_{\mathrm{D}}^{23}+7.13\left(c 0.89, \mathrm{CHCl}_{3}\right.$ ); IR (film) 3480, 3064, 2986, 2938, 1769, 1721, 1385, 1274, 1218, 1108, 1071, $713 \mathrm{~cm}^{-1} ;{ }^{1} \mathrm{H} \mathrm{NMR}\left(400 \mathrm{MHz}, \mathrm{CDCl}_{3}\right) \delta 1.23$ ( $3 \mathrm{H}, \mathrm{s}, \mathrm{CH}_{3}$ of acetonide), $1.31\left(3 \mathrm{H}, \mathrm{s}, \mathrm{CH}_{3}\right.$ of acetonide), $1.35\left(3 \mathrm{H}, \mathrm{s}, \mathrm{CH}_{3}\right.$ of acetonide), 1.43 (3H, s, $\mathrm{CH}_{3}$ of acetonide), $1.99(1 \mathrm{H}$, brs, $\mathrm{OH}), 2.06\left(3 \mathrm{H}, \mathrm{s}, \mathrm{CH}_{3} \mathrm{CO}\right), 3.72-3.79(2 \mathrm{H}, \mathrm{m}, \mathrm{H} 6, \mathrm{H} 9)$, 4.03-4.05 (3H, m, H7, H8, H10), 4.34 (1H, dd, $J=11.9,6.9 \mathrm{~Hz}, \mathrm{H} 11 \mathrm{a}), 4.44$ (1H, dd, $J=11.9$, $2.7 \mathrm{~Hz}, \mathrm{H} 11 \mathrm{~b}), 4.64$ (1H, dd, $J=10.1,10.1 \mathrm{~Hz}, \mathrm{H} 5), 4.71$ (1H, dd, $J=10.1,10.1 \mathrm{~Hz}, \mathrm{H} 4), 5.60$ $(1 \mathrm{H}, \mathrm{dd}, J=10.1,10.1 \mathrm{~Hz}, \mathrm{H} 2), 6.13(1 \mathrm{H}, \mathrm{d}, J=10.1 \mathrm{~Hz}, \mathrm{H} 1), 6.38(1 \mathrm{H}, \mathrm{dd}, J=10.1,10.1 \mathrm{~Hz}$, H3), 7.26-7.30 (3H, m, aromatic), $7.37(2 \mathrm{H}, \mathrm{t}, J=7.8 \mathrm{~Hz}$, aromatic), 7.40-7.44 (3H, m, aromatic), $7.51(1 \mathrm{H}, \mathrm{t}, J=7.3 \mathrm{~Hz}$, aromatic), $7.55(1 \mathrm{H}, \mathrm{t}, J=7.8 \mathrm{~Hz}$, aromatic), 7.65-7.67 (2H, $\mathrm{m}$, aromatic), 7.76-7.78 (3H, m, aromatic), $7.92(2 \mathrm{H}, \mathrm{d}, J=7.3 \mathrm{~Hz}$, aromatic), 8.01 (2H, d, $J=$ $7.3 \mathrm{~Hz}$, aromatic); ${ }^{13} \mathrm{C} \mathrm{NMR}\left(100 \mathrm{MHz}, \mathrm{CDCl}_{3}\right): \delta 20.8,23.4,23.8,24.5,24.7,53.9,64.7,66.5$, 67.1, 68.0, 68.7, 69.9, 70.6, 71.7, 72.6, 91.8, 101.2, 101.6, 123.6 (2C), 128.30 (2C), 128.32 (3C), 128.42 (2C), 128.53, 128.8, 129.6 (2C), 129.77 (2C), 129.81 (2C), 129.93, 131.5, 133.0, 133.3, 133.4, 134.0 (2C), 165.0, 165.5, 166.3, 168.2 (2C), 168.8; HRMS (ESI-TOF) calcd for $\mathrm{C}_{48} \mathrm{H}_{47} \mathrm{NO}_{16} \mathrm{Na}[\mathrm{M}+\mathrm{Na}]^{+}$916.2787, found 916.2783.

6- $\beta$ : colorless amorphous; $[\alpha]_{\mathrm{D}}{ }^{26}-3.21\left(c\right.$ 1.07, $\mathrm{CHCl}_{3}$ ); IR (film) 3524, 3067, 2990, 2934, 1770, 
1725, 1378, 1270, $1099 \mathrm{~cm}^{-1}$; ${ }^{1} \mathrm{H}$ NMR (400 MHz, $\left.\mathrm{CDCl}_{3}\right) \delta 1.26\left(3 \mathrm{H}, \mathrm{s}, \mathrm{CH}_{3}\right.$ of acetonide), $1.34\left(3 \mathrm{H}, \mathrm{s}, \mathrm{CH}_{3}\right.$ of acetonide), $1.40\left(3 \mathrm{H}, \mathrm{s}, \mathrm{CH}_{3}\right.$ of acetonide), 1.42 (3H, s, $\mathrm{CH}_{3}$ of acetonide), $2.06\left(3 \mathrm{H}, \mathrm{s}, \mathrm{CH} \mathrm{CO}_{3} \mathrm{CO}, 2.48\right.$ (1H, brs, $\left.\mathrm{OH}\right), 3.54$ (1H, d, $\left.J=8.7 \mathrm{~Hz}, \mathrm{H} 6\right), 3.88$ (1H, m, H9), 3.943.99 (2H, m, H7, H8), 4.06 (1H, m , H10), 4.37 (1H, dd, J =11.9, 7.3 Hz, H11a), 4.45 (1H, dd, $J=11.9,2.8 \mathrm{~Hz}, \mathrm{H} 11 \mathrm{~b}), 4.90(1 \mathrm{H}, \mathrm{d}, J=10.5 \mathrm{~Hz}, \mathrm{H} 5), 5.03$ (1H, dd, $J=10.5,10.5 \mathrm{~Hz}, \mathrm{H} 4)$, $5.65(1 \mathrm{H}, \mathrm{dd}, J=10.5,10.5 \mathrm{~Hz}, \mathrm{H} 2), 6.17(1 \mathrm{H}, \mathrm{d}, J=10.5 \mathrm{~Hz}, \mathrm{H} 1), 6.30$ (1H, dd, $J=10.5,10.5$ $\mathrm{Hz}, \mathrm{H} 3$ ), 7.24-7.28 (3H, m, aromatic), 7.34-7.44 (4H, m, aromatic), 7.50-7.56 (2H, m, aromatic), 7.65-7.76 (5H, m, aromatic), 7.88-7.93 (3H, m, aromatic), $8.01(2 \mathrm{H}, \mathrm{d}, J=7.3 \mathrm{~Hz}$, aromatic); ${ }^{13} \mathrm{C}$ NMR $\left(100 \mathrm{MHz} \mathrm{CDCl}_{3}\right): \delta 20.7,23.5,24.0,24.2,24.4,49.9,64.5,66.2,68.1(2 \mathrm{C}), 70.2$, 71.0, 71.1, 71.47, 71.50, 91.9, 101.3, 101.4, 123.7 (2C), 128.25 (2C), $128.29(3 \mathrm{C}), 128.37$ (2C), 128.42, 128.8, 129.5 (2C), 129.7 (3C), 129.9, 131.2, 131.5, 133.0, 133.2, 133.4, 134.1, 134.3, 164.9, 165.7, 166.3, 167.0, 168.0, 168.8; HRMS (ESI-TOF) calcd for $\mathrm{C}_{48} \mathrm{H}_{47} \mathrm{NO}_{16} \mathrm{Na}[\mathrm{M}+\mathrm{Na}]^{+}$ 916.2787, found 916.2787 .
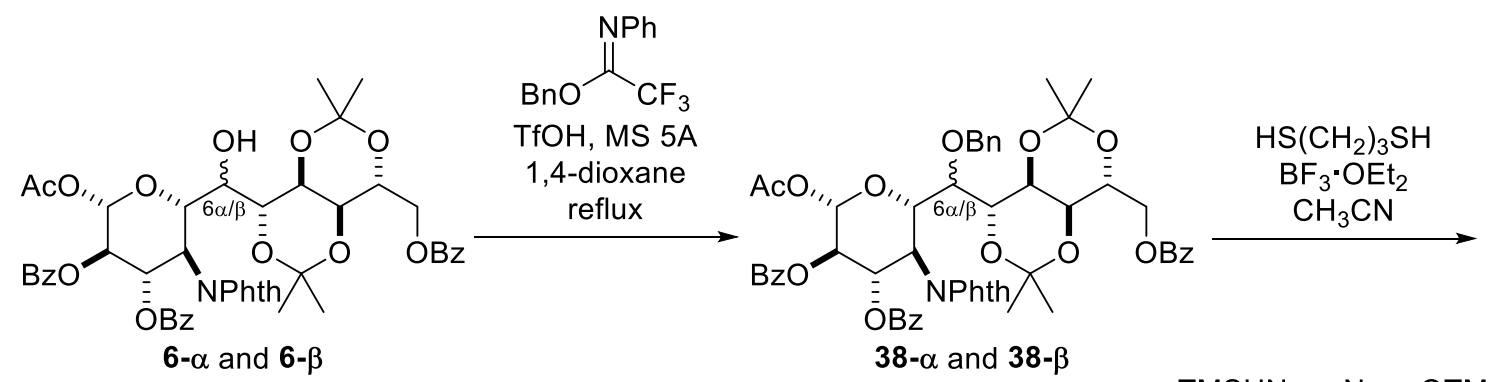

38- $\alpha$ and $38-\beta$
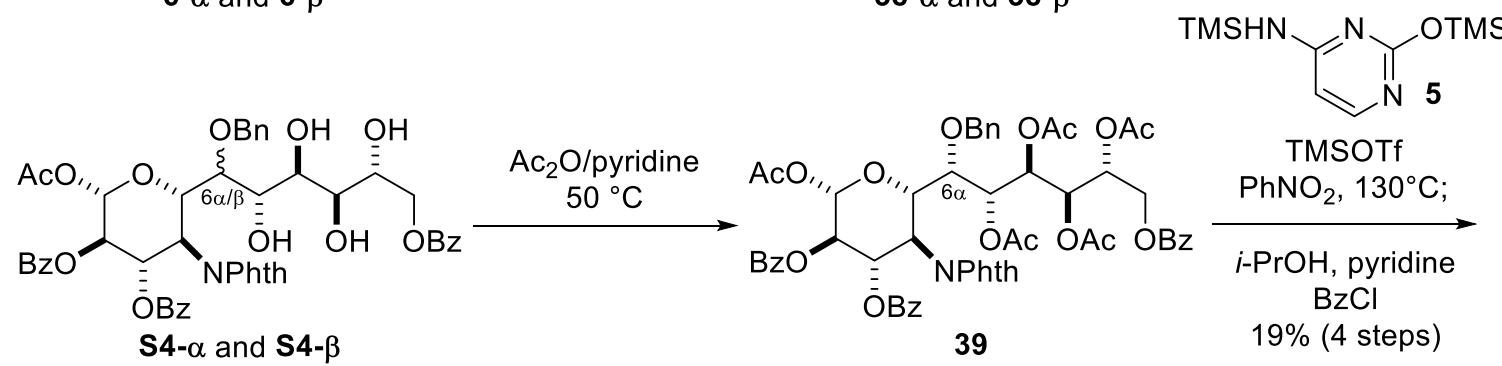

TMSOTf $\mathrm{PhNO}_{2}, 130^{\circ} \mathrm{C}$

i-PrOH, pyridine $\mathrm{BzCl}$ $19 \%$ (4 steps)

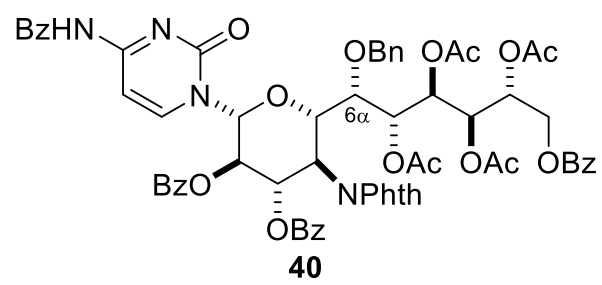

Benzoylcytosine 40. Molecular sieves $5 \mathrm{~A}(750 \mathrm{mg})$ was added to a solution of a $2.2: 1$ diastereomeric mixture of alcohol $6(150 \mathrm{mg}, 168 \mu \mathrm{mol})$ and $N$-phenyl-2,2,2trifluoroacetimidate $(194 \mu \mathrm{L}, 841 \mu \mathrm{mol})$ in 1,4 -dioxane $(5.50 \mathrm{~mL})$ at $25{ }^{\circ} \mathrm{C}$. The reaction mixture was stirred at $25^{\circ} \mathrm{C}$ for $15 \mathrm{~min}$. A solution of $\mathrm{TfOH}(0.34 \mathrm{M}$ in 1,4-dioxane, $100 \mu \mathrm{L}$, 
$34 \mu \mathrm{mol}$ ) was then added at $25^{\circ} \mathrm{C}$. After the reaction mixture was heated to reflux for $1.5 \mathrm{~h}$, $\mathrm{Et}_{3} \mathrm{~N}(100 \mu \mathrm{L})$ was added to the reaction mixture at $0{ }^{\circ} \mathrm{C}$. The resultant mixture was filtered through a pad of Celite with EtOAc $(6 \mathrm{~mL})$. After saturated aqueous $\mathrm{NaHCO}_{3}(6 \mathrm{~mL})$ was added to the filtrate, the resultant mixture was extracted with EtOAc $(6 \mathrm{~mL} \times 3)$. The combined organic layers were dried over $\mathrm{Na}_{2} \mathrm{SO}_{4}$, filtered, and concentrated. The residue was purified by flash column chromatography ( $30 \mathrm{~g}$, hexane/EtOAc $=5 / 1$ to $1 / 1)$ to afford a $1.6: 1$ diastereomeric mixture of the crude benzyl ether 38- $\boldsymbol{\alpha}$ and $\mathbf{3 8}-\boldsymbol{\beta}$ (102 $\mathrm{mg}$ ), which was used in the next reaction without further purification. HRMS (ESI-TOF) calcd for $\mathrm{C}_{55} \mathrm{H}_{53} \mathrm{NO}_{16} \mathrm{Na}$ $[\mathrm{M}+\mathrm{Na}]^{+} 1006.3257$, found 1006.3247 .

A solution of $\mathrm{BF}_{3} \cdot \mathrm{OEt}_{2}\left(0.55 \mathrm{M}\right.$ in $\left.\mathrm{CH}_{3} \mathrm{CN}, 100 \mu \mathrm{L}, 55 \mu \mathrm{mol}\right)$ was added to a solution of the above crude benzyl ether 38 (102 mg) and 1,3-propanedithiol $(418 \mu \mathrm{L}, 4.16 \mathrm{mmol})$ in $\mathrm{CH}_{3} \mathrm{CN}$ $(1.98 \mathrm{~mL})$ at $25{ }^{\circ} \mathrm{C}$. After the reaction mixture was stirred at $25{ }^{\circ} \mathrm{C}$ for $40 \mathrm{~min}$, saturated aqueous $\mathrm{NaHCO}_{3}(4 \mathrm{~mL})$ was added. The resultant mixture was then extracted with EtOAc (4 mL x5). The combined organic layers were dried over $\mathrm{Na}_{2} \mathrm{SO}_{4}$, filtered, and concentrated to afford the crude tetraol $\mathbf{S 4}(550 \mathrm{mg})$, which was used in the next reaction without further purification. ${ }^{\mathrm{S}} 6$ HRMS (ESI-TOF) calcd for $\mathrm{C}_{49} \mathrm{H}_{45} \mathrm{NO}_{16} \mathrm{Na}[\mathrm{M}+\mathrm{Na}]^{+}$926.2631, found 926.2620.

A solution of the above crude tetraol $\mathbf{S 4}(550 \mathrm{mg})$ in pyridine $(1.73 \mathrm{~mL})$ and $\mathrm{Ac}_{2} \mathrm{O}(1.73 \mathrm{~mL})$ was stirred at $50{ }^{\circ} \mathrm{C}$ for $5 \mathrm{~h}$. Saturated aqueous $\mathrm{NaHCO}_{3}(8 \mathrm{~mL})$ and EtOAc $(8 \mathrm{~mL})$ were then added to the reaction mixture at $0{ }^{\circ} \mathrm{C}$. After being stirred vigorously for $20 \mathrm{~min}$, the resultant mixture was extracted with EtOAc $(8 \mathrm{~mL} \times 3)$. The combined organic layers were washed with saturated aqueous $\mathrm{NaHCO}_{3}(20 \mathrm{~mL})$, dried over $\mathrm{Na}_{2} \mathrm{SO}_{4}$, and concentrated. The residue was purified by automated flash column chromatography (hexane/EtOAc $=65 / 35$ to $46 / 54$ ) to afford the crude pentaacetate $39(62.2 \mathrm{mg}) .{ }^{\mathrm{S} 6} \quad$ To collect the analytical data, 39 was partially purified by PTLC $(10 \mathrm{~cm} \times 20 \mathrm{~cm}, 2$ plates, hexane/EtOAc $=3 / 2)$.

39: pale yellow amorphous; $[\alpha]_{\mathrm{D}}{ }^{17}+15.2\left(c\right.$ 0.98, $\left.\mathrm{CHCl}_{3}\right)$; IR (film) 2963, 1731, 1602, 1451, 1373, 1274, 1218, 1068, 1031, $713 \mathrm{~cm}^{-1} ;{ }^{1} \mathrm{H}$ NMR (400 MHz, $\left.\mathrm{CDCl}_{3}\right) \delta 1.56\left(3 \mathrm{H}, \mathrm{s}, \mathrm{CH}_{3} \mathrm{CO}\right)$, $2.03\left(3 \mathrm{H}, \mathrm{s}, \mathrm{CH} \mathrm{COO}_{3}, 2.04\left(6 \mathrm{H}, \mathrm{s}, \mathrm{CH}_{3} \mathrm{CO}\right), 2.22\left(3 \mathrm{H}, \mathrm{s}, \mathrm{CH}_{3} \mathrm{CO}\right), 3.30(1 \mathrm{H}, \mathrm{d}, J=4.1,1.4 \mathrm{~Hz}\right.$, H6), $4.13(1 \mathrm{H}, \mathrm{dd}, J=12.8,5.5 \mathrm{~Hz}, \mathrm{H} 11 \mathrm{a}), 4.48(1 \mathrm{H}, \mathrm{d}, J=11.4 \mathrm{~Hz}, \mathrm{OCHaHbPh}), 4.53(1 \mathrm{H}$, $\mathrm{d}, J=11.4 \mathrm{~Hz}, \mathrm{OCHa} H \mathrm{bPh}), 4.56(1 \mathrm{H}, \mathrm{dd}, J=12.8,2.3 \mathrm{~Hz}, \mathrm{H} 11 \mathrm{~b}), 5.02(1 \mathrm{H}, \mathrm{dd}, J=9.6,9.6$ Hz, H4), 5.07-5.14 (2H, m, H5, H10), 5.37 (1H, dd, $J=9.6,1.4$ Hz, H8), 5.52 (1H, dd, J = 9.6, $1.8 \mathrm{~Hz}, \mathrm{H} 9), 5.57(1 \mathrm{H}, \mathrm{dd}, J=8.7,8.7 \mathrm{~Hz}, \mathrm{H} 2), 5.72(1 \mathrm{H}, \mathrm{dd}, J=9.6,1.4 \mathrm{~Hz}, \mathrm{H} 7), 6.01(1 \mathrm{H}, \mathrm{d}$, $J=8.7 \mathrm{~Hz}, \mathrm{H} 1), 6.21(1 \mathrm{H}, \mathrm{dd}, J=9.6,8.7 \mathrm{~Hz}, \mathrm{H} 3), 7.17-8.03$ (24H, m, aromatic), Detectable signals of ${ }^{13} \mathrm{C} \mathrm{NMR}\left(100 \mathrm{MHz}, \mathrm{CDCl}_{3}\right): \delta 19.9,20.73,20.79,20.85,21.0,51.2,62.4,66.8,67.3$, 
$67.7,68.2,69.8,70.9,71.1,72.6,75.2,92.3,123.5,123.6,127.6,127.8,128.20,128.27,128.37$, $128.39,128.8,129.7,129.81,129.84,133.1,133.3,133.4,134.2,134.3,137.0,164.8,165.6$, 166.0, 166.6, 167.7, 168.6, 169.6, 170.0, 170.1, 170.5; HRMS (ESI-TOF) calcd for $\mathrm{C}_{57} \mathrm{H}_{53} \mathrm{NO}_{20} \mathrm{Na}[\mathrm{M}+\mathrm{Na}]^{+}$1094.3053, found 1094.3040.

Cytosine $(350 \mathrm{mg}, 3.15 \mathrm{mmol})$ and $\left(\mathrm{NH}_{4}\right)_{2} \mathrm{SO}_{4}(10.4 \mathrm{mg}, 78.7 \mu \mathrm{mol})$ was dissolved in $\left(\mathrm{Me}_{3} \mathrm{Si}\right)_{2} \mathrm{NH}(3.50 \mathrm{~mL})$. After being refluxed for $2 \mathrm{~h}$, the reaction mixture was concentrated. The residue was dissolved in toluene $(4 \mathrm{~mL})$ and filtered. The filtrate was azeotroped with toluene $(5 \mathrm{~mL} \times 3)$ to afford the crude $5^{\mathrm{S} 7}$, which was used immediately in the next reaction.

TMSOTf $(34.6 \mu \mathrm{L}, 192 \mu \mathrm{mol})$ was added to a solution of the above pentaacetate $\mathbf{3 9}(62.2 \mathrm{mg})$ and $5(44.5 \mathrm{mg}, 174 \mu \mathrm{mol})$ in $\mathrm{PhNO}_{2}(580 \mu \mathrm{L})$ at $130{ }^{\circ} \mathrm{C}$. After the reaction mixture was stirred at $130{ }^{\circ} \mathrm{C}$ for $5 \mathrm{~h}$, pyridine $(224 \mu \mathrm{L}, 2.90 \mathrm{mmol}), i$-PrOH $(14.7 \mu \mathrm{L}, 192 \mu \mathrm{mol})$, and $\mathrm{CH}_{2} \mathrm{Cl}_{2}(580 \mu \mathrm{L})$ were added to the mixture at $25^{\circ} \mathrm{C}$. The resultant mixture was stirred for 10 min at $25^{\circ} \mathrm{C}$ and then $\mathrm{BzCl}(67.4 \mu \mathrm{L}, 580 \mu \mathrm{mol})$ was added to the mixture. After the reaction mixture was stirred at $25^{\circ} \mathrm{C}$ for $1 \mathrm{~h}$, saturated aqueous $\mathrm{NaHCO}_{3}(5 \mathrm{~mL})$ was added. The resultant mixture was extracted with $\mathrm{CHCl}_{3}(5 \mathrm{~mL}$ x4). The combined organic layers were dried over $\mathrm{Na}_{2} \mathrm{SO}_{4}$ and concentrated. The residue was filtered through a pad of silica gel $(6 \mathrm{~g}, \mathrm{EtOAc})$. The filtrate was concentrated, and the residue was purified by automated flash column chromatography (hexane/EtOAc $=50 / 50$ to 25/75) to afford benzoylcytosine 40 (40.0 $\mathrm{mg}, 32.6 \mu \mathrm{mol})$ in $19 \%$ yield over 4 steps from a 2.2:1 diastereomeric mixture of $\mathbf{6}-\boldsymbol{\alpha}$ and $\mathbf{6}-\boldsymbol{\beta}$ : pale yellow amorphous; $[\alpha]_{\mathrm{D}}{ }^{28}+9.26\left(c 1.47, \mathrm{CHCl}_{3}\right)$; IR (film) 3065, 2959, 1731, 1629, 1555 , 1483, 1376, 1220, 1069, 855, 798, $712 \mathrm{~cm}^{-1} ;{ }^{1} \mathrm{H}$ NMR (400 MHz, $\left.\mathrm{CDCl}_{3}\right) \delta 1.31(3 \mathrm{H}, \mathrm{s}$, $\left.\mathrm{CH}_{3} \mathrm{CO}\right), 2.02\left(3 \mathrm{H}, \mathrm{s}, \mathrm{CH}_{3} \mathrm{CO}\right), 2.04\left(3 \mathrm{H}, \mathrm{s}, \mathrm{CH}_{3} \mathrm{CO}\right), 2.28\left(3 \mathrm{H}, \mathrm{s}, \mathrm{CH}_{3} \mathrm{CO}\right), 3.30(1 \mathrm{H}, \mathrm{d}, J=$ 3.0 Hz, H6), 4.10 (1H, dd, $J=12.4,5.2 \mathrm{~Hz}, \mathrm{H} 11 \mathrm{a}), 4.45$ (1H, d, $J=11.2 \mathrm{~Hz}, \mathrm{OCHaHbPh}), 4.56$ $(1 \mathrm{H}, \mathrm{dd}, J=12.4,2.5 \mathrm{~Hz}, \mathrm{H} 11 \mathrm{~b}), 4.57(1 \mathrm{H}, \mathrm{d}, J=11.2 \mathrm{~Hz}, \mathrm{OCHa} H \mathrm{bPh}), 5.05$ (1H, ddd, $J=9.6$, 5.2, $2.5 \mathrm{~Hz}, \mathrm{H} 10), 5.16$ (1H, dd, $J=9.6,9.6 \mathrm{~Hz}, \mathrm{H} 4), 5.26-5.29$ (2H, m, H5, H7), 5.49 (1H, d, $J=9.6 \mathrm{~Hz}, \mathrm{H} 9), 5.64$ (1H, d, $J=9.6 \mathrm{~Hz}, \mathrm{H} 8), 5.69$ (1H, dd, $J=9.6,9.6 \mathrm{~Hz}, \mathrm{H} 2), 6.30$ (1H, dd, $J=9.6,9.6 \mathrm{~Hz}, \mathrm{H} 3), 6.44(1 \mathrm{H}, \mathrm{brd}, J=9.6 \mathrm{~Hz}, \mathrm{H} 1), 7.25-8.02(31 \mathrm{H}, \mathrm{m}$, aromatic, H18, H19), $8.62(1 \mathrm{H}$, brs, $\mathrm{NH})$; Detectable signals of ${ }^{13} \mathrm{C} \mathrm{NMR}\left(100 \mathrm{MHz}, \mathrm{CDCl}_{3}\right)$ : $\delta 19.4,20.7,20.8,21.7$, $50.8,62.3,66.3,67.0,67.6,68.1,71.1,72.7,72.9,74.3,82.0,97.2,123.76,123.81,127.5,127.9$, 128.0, 128.1, 128.4, 129.1, 129.5, 129.7, 129.8, 130.0, 131.0, 131.2, 133.2, 133.4, 133.6, 134.5, 134.7, 136.9, 145.0, 154.4, 162.4, 165.1, 165.4, 166.0, 166.4, 167.7, 169.4, 170.0, 170.1, 170.6; HRMS (ESI-TOF) calcd for $\mathrm{C}_{66} \mathrm{H}_{58} \mathrm{~N}_{4} \mathrm{O}_{20} \mathrm{Na}[\mathrm{M}+\mathrm{Na}]^{+}$1249.3537, found 1249.3520. 

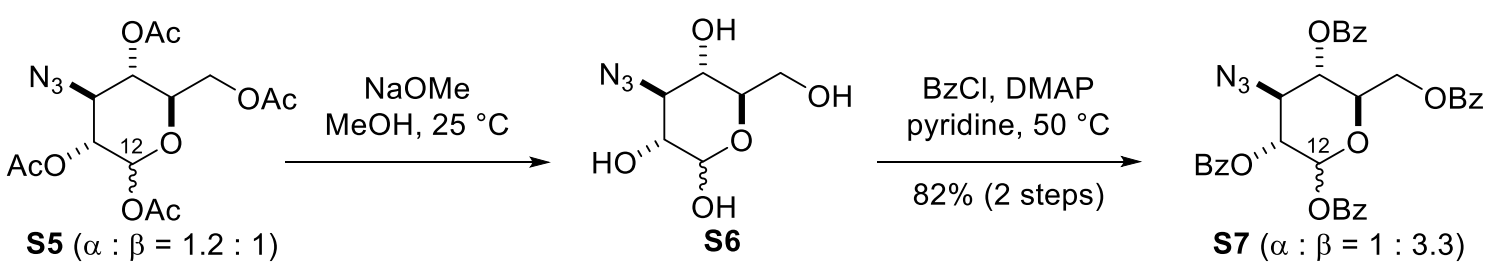

Tetrabenzoate S7. A solution of a $1.2: 1$ diastereomeric mixture of tetraacetates S5- $\alpha$ [CAS: 151073-01-7] ${ }^{\mathrm{S} 8}$ and S5- $\beta$ [CAS: 80065-02-7] ${ }^{\mathrm{S} 9}$ (154 mg, $\left.413 \mu \mathrm{mol}\right)$ and NaOMe $(25 \mathrm{wt} \%$ in $\mathrm{MeOH}, 357 \mu \mathrm{L}, 1.65 \mathrm{mmol})$ in $\mathrm{MeOH}(4.13 \mathrm{~mL})$ was stirred at $25^{\circ} \mathrm{C}$ for $3 \mathrm{~h}$. After being neutralized with $0.5 \mathrm{M} \mathrm{HCl}$ in $\mathrm{MeOH}(3.3 \mathrm{~mL}, 1.65 \mathrm{mmol})$, the reaction mixture was concentrated to afford the crude tetraol S6 [CAS: 104875-44-7] ${ }^{\mathrm{S} 10}(252 \mathrm{mg})$, which was used in the next reaction without further purification.

$\mathrm{BzCl}(288 \mu \mathrm{L}, 2.48 \mathrm{mmol})$ was added to a solution of the above crude tetraol S6 $(252 \mathrm{mg})$ and DMAP $(2.5 \mathrm{mg}, 21 \mu \mathrm{mol})$ in pyridine $(2.06 \mathrm{~mL})$ at $25{ }^{\circ} \mathrm{C}$. After the reaction mixture was stirred at $50{ }^{\circ} \mathrm{C}$ for $4 \mathrm{~h}, \mathrm{H}_{2} \mathrm{O}(5 \mathrm{~mL})$ was added to the reaction mixture. The resultant mixture was extracted with $\mathrm{CH}_{2} \mathrm{Cl}_{2}\left(5 \mathrm{~mL}\right.$ x3). The combined organic layers were dried over $\mathrm{Na}_{2} \mathrm{SO}_{4}$ and concentrated. The residue was purified by flash column chromatography on silica gel (5 $\mathrm{g}$, hexane/EtOAc $=10 / 1$ to $4 / 1)$ and on amine-modified silica gel $(5 \mathrm{~g}$, hexane/EtOAc $=10 / 1$ to $2 / 1$ ) to afford a $1: 3.3$ diastereomeric mixture of pentabenzoate $\mathbf{S 7}-\boldsymbol{\alpha}$ and $\mathbf{S 7}-\boldsymbol{\beta}$ (210 $\mathrm{mg}, 338$ $\mu \mathrm{mol}$ ) in 82\% yield over 2 steps: colorless solid; IR (film) 3446, 3335, 3066, 2964, 2913, 2107 , 1734, 1261, $1076 \mathrm{~cm}^{-1} ;{ }^{1} \mathrm{H}$ NMR (400 MHz, $\left.\mathrm{CDCl}_{3}\right): \delta 4.22(0.77 \mathrm{H}, \mathrm{t}, J=10.1 \mathrm{~Hz}, \mathrm{H} 14), 4.32$ (0.77H, m, H16), 4.41-4.54 (1.46H, m, H14, H16, H17a), 4.65 (1.00H, m, H17b), 5.45 (0.23H, dd, $J=11.0,3.7 \mathrm{~Hz}, \mathrm{H} 13), 5.59(1.00 \mathrm{H}, \mathrm{m}, \mathrm{H} 15), 5.65(0.77 \mathrm{H}, \mathrm{dd}, J=8.7,8.2 \mathrm{~Hz}, \mathrm{H} 13), 6.21$ $(0.77 \mathrm{H}, \mathrm{d}, J=8.7 \mathrm{~Hz}, \mathrm{H} 12), 6.81(0.23 \mathrm{H}, \mathrm{d}, J=3.2 \mathrm{~Hz}, \mathrm{H} 12), 7.38-7.49$ (8.00H, m, aromatic), 7.54-7.69 $\left(4.00 \mathrm{H}, \mathrm{m}\right.$, aromatic), 7.99-8.13 $\left(8.00 \mathrm{H}, \mathrm{m}\right.$, aromatic); Detectable signals of ${ }^{13} \mathrm{C}$ NMR (100 MHz, $\left.\mathrm{CDCl}_{3}\right): \delta 61.8,62.4,62.6,64.7,68.8,69.1,70.2,70.7,70.8,73.7,89.4,92.6$, $128.23,128.28,128.32,128.48,128.50,128.56,128.7,128.9,129.4,129.70,129.74,129.82$, $129.91,129.94,130.1,133.08,133.12$, 133.7, 133.8, 133.85, 133.92, 164.1, 164.6, 164.79, 164.80, 164.82, 165.0, 166.02, 166.04; HRMS (ESI-TOF) calcd for $\mathrm{C}_{34} \mathrm{H}_{27} \mathrm{~N}_{3} \mathrm{O}_{9} \mathrm{Na}[\mathrm{M}+\mathrm{Na}]^{+}$ 644.1640 , found 644.1647 .

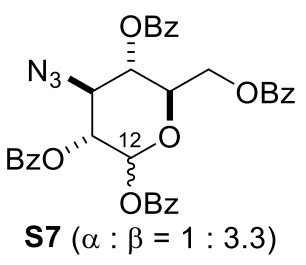

Hemiacetal S8 [CAS: 1846590-32-6]. ${ }^{\text {S11 }}$

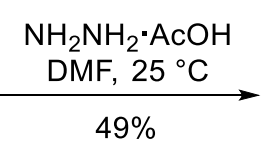

A solution of a $1: 3.3$ mixture of benzoylacetal S7- 
$\boldsymbol{\alpha}$ and S7- $\boldsymbol{\beta}(110 \mathrm{mg}, 177 \mu \mathrm{mol}), \mathrm{N}_{2} \mathrm{H}_{4} \cdot \mathrm{H}_{2} \mathrm{O}(17.2 \mu \mathrm{L}, 354 \mu \mathrm{mol})$, and $\mathrm{AcOH}(20.3 \mu \mathrm{L}, 354$ $\mu \mathrm{mol})$ in DMF $(885 \mu \mathrm{L})$ was stirred at $25^{\circ} \mathrm{C}$ for $40 \mathrm{~min}$. After $\mathrm{H}_{2} \mathrm{O}(4 \mathrm{~mL})$ was added to the reaction mixture, the resultant mixture was extracted with EtOAc $(4 \mathrm{~mL} \times 3)$. The combined organic layers were dried over $\mathrm{Na}_{2} \mathrm{SO}_{4}$ and concentrated. The residue was purified by flash column chromatography on silica gel $(10 \mathrm{~g}$, hexane/EtOAc $=10 / 1$ to $3 / 1)$ to afford hemiacetal S8 (45.2 mg, $87.3 \mu \mathrm{mol})$ in 49\% yield: colorless solid; m.p. $187-189^{\circ} \mathrm{C}:[\alpha]_{\mathrm{D}}{ }^{24}+97.7(c 1.43$, $\mathrm{CHCl}_{3}$ ); IR (film) 3479, 3069, 2952, 2359, 2109, 1716, $1267 \mathrm{~cm}^{-1} ;{ }^{1} \mathrm{H}$ NMR (400 MHz, $\left.\mathrm{CDCl}_{3}\right)$ : $\delta 3.73(1 \mathrm{H}, \mathrm{d}, J=3.2 \mathrm{~Hz}, \mathrm{OH}), 4.33(1 \mathrm{H}, \mathrm{dd}, J=11.9,4.1 \mathrm{~Hz}, \mathrm{H} 17 \mathrm{a}), 4.44$ (1H, dd, $J=10.1$, $10.1 \mathrm{~Hz}, \mathrm{H} 14), 4.54$ (1H, ddd, $J=10.1,4.1,2.8 \mathrm{~Hz}, \mathrm{H} 16), 4.61$ (1H, dd, $J=11.9,2.8 \mathrm{~Hz}, \mathrm{H} 17 \mathrm{~b})$, $5.04(1 \mathrm{H}, \mathrm{dd}, J=10.1,3.2 \mathrm{~Hz}, \mathrm{H} 13), 5.40(1 \mathrm{H}, \mathrm{dd}, J=10.1,10.1 \mathrm{~Hz}, \mathrm{H} 15), 5.68$ (1H, m, $J=$ 3.2, 3.2 Hz, H12), 7.37 (2H, t, $J=7.8 \mathrm{~Hz}$, aromatic), 7.44-7.48 (4H, m. aromatic), 7.52 (1H, t, $J=7.3 \mathrm{~Hz}$, aromatic), $7.60(2 \mathrm{H}, \mathrm{t}, . J=7.8 \mathrm{~Hz}$, aromatic), $8.01(2 \mathrm{H}, \mathrm{d}, J=7.8 \mathrm{~Hz}), 8.09(4 \mathrm{H}, \mathrm{m}$, aromatic) ${ }^{13} \mathrm{C} \mathrm{NMR}\left(100 \mathrm{MHz}, \mathrm{CDCl}_{3}\right): \delta 61.3,62.8,67.5,69.4,72.7,89.7,128.3$ (3C), 128.6 (3C), 128.8, 128.9, 129.4, 129.8 (3C), 129.9 (3C), 133.2, 133.67, 133.68, 165.0, 165.6, 166.4; HRMS (ESI-TOF) calcd for $\mathrm{C}_{27} \mathrm{H}_{23} \mathrm{~N}_{3} \mathrm{O}_{8} \mathrm{Na}[\mathrm{M}+\mathrm{Na}]^{+}$540.1377, found 540.1370.

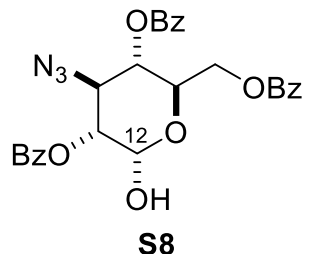

S8
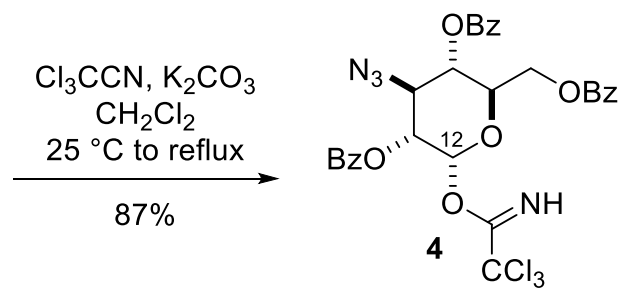

Trichloroacetimidate 4 [CAS: 1846590-34-8]. ${ }^{\mathrm{S} 11}$ Trichloroacetonitrile $\left(\mathrm{Cl}_{3} \mathrm{CCN}, 198 \mu \mathrm{L}\right.$ $1.97 \mathrm{mmol})$ was added to a solution of hemiacetal $\mathbf{S 8}(102 \mathrm{mg}, 197 \mu \mathrm{mol})$ and $\mathrm{K}_{2} \mathrm{CO}_{3}(272 \mathrm{mg}$, $1.97 \mathrm{mmol})$ in $\mathrm{CH}_{2} \mathrm{Cl}_{2}(1.97 \mathrm{~mL})$ at $25^{\circ} \mathrm{C}$. After the reaction mixture was stirred at $25^{\circ} \mathrm{C}$ for $11 \mathrm{~h}$, the reaction mixture was heated to reflux for $1 \mathrm{~h}$. Then, the reaction mixture was filtered through a pad of Celite with $\mathrm{CH}_{2} \mathrm{Cl}_{2}(2 \mathrm{~mL})$ and concentrated. The residue was purified by flash column chromatography on silica gel $(10 \mathrm{~g}$, hexane/EtOAc $=25 / 1$ to $5 / 1)$ to afford trichloroacetimidate $4(113 \mathrm{mg}, 171 \mu \mathrm{mol})$ in $87 \%$ yield: colorless amorphous; $[\alpha]_{\mathrm{D}}{ }^{24}+94.54$ (c 1.42, $\mathrm{CHCl}_{3}$ ); IR (film) 3336, 3069, 2957, 2355, 1730, 1681, 1266, 1099, $1022 \mathrm{~cm}^{-1} ;{ }^{1} \mathrm{H}$ NMR (400 MHz, $\left.\mathrm{CDCl}_{3}\right): \delta 4.40(1 \mathrm{H}, \mathrm{dd}, J=12.4,5.0 \mathrm{~Hz}, \mathrm{H} 17 \mathrm{a}), 4.45(1 \mathrm{H}, \mathrm{dd}, J=10.0,10.0$ Hz, H14), 4.50 (1H, ddd, $J=10.0,5.0,2.8 \mathrm{~Hz}, \mathrm{H} 16), 4.60$ (1H, dd, $J=12.4,2.8 \mathrm{~Hz}, \mathrm{H} 17 \mathrm{~b})$, $5.36(1 \mathrm{H}, \mathrm{dd}, J=10.5,3.6 \mathrm{~Hz}, \mathrm{H} 13), 5.51(1 \mathrm{H}, \mathrm{dd}, J=10.0,10.0 \mathrm{~Hz}, \mathrm{H} 15), 6.74(1 \mathrm{H}, \mathrm{d}, J=3.2$ $\mathrm{Hz}, \mathrm{H} 12)$, 7.38-7.49 (6H, m, aromatic), 7.53-7.63 (3H, m, aromatic), 8.00-8.10 (6H, m, aromatic), $8.65(1 \mathrm{H}, \mathrm{s}, \mathrm{NH}) ;{ }^{13} \mathrm{C} \mathrm{NMR}\left(100 \mathrm{MHz}, \mathrm{CDCl}_{3}\right): \delta 61.7,62.4,68.6,70.4,71.0,90.7$, 
92.5, 128.35 (2C), 128.48, $128.56(3 \mathrm{C}), 128.59$ (2C), 129.4, 129.7 (2C), $129.9(2 \mathrm{C}), 130.0(2 \mathrm{C})$, 133.2, 133.79, 133.84, 160.2, 164.9, 165.1, 166.0; HRMS (ESI-TOF) calcd for $\mathrm{C}_{29} \mathrm{H}_{23} \mathrm{Cl}_{3} \mathrm{~N}_{4} \mathrm{O}{ }_{8} \mathrm{Na}[\mathrm{M}+\mathrm{Na}]^{+}$683.0474, found 683.0471.

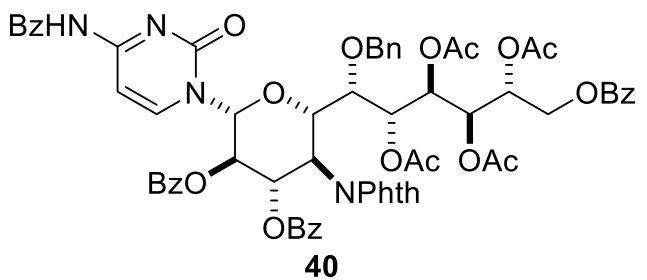

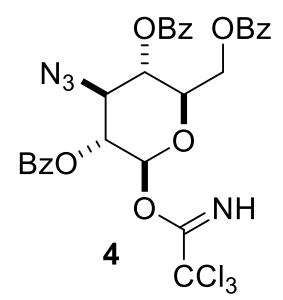

$61 \%$ (2 steps)

$(42: 12-e p i-42=1.8: 1)$

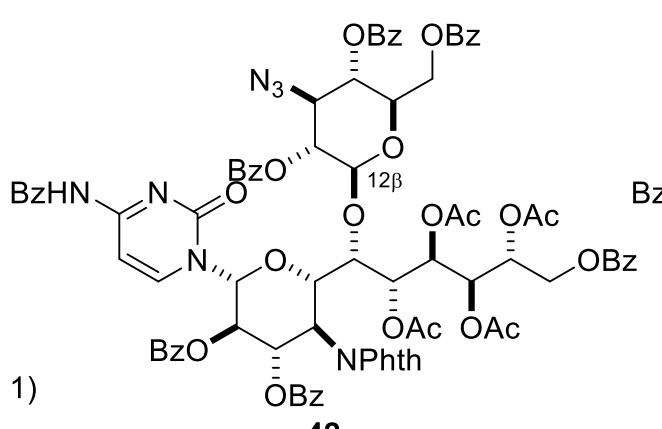

42

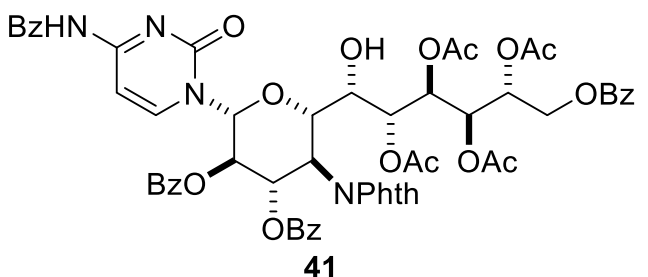

41

Fully protected hikizimycin 42 and 12-epi-42. A solution of benzoylcytosine $40(49.7 \mathrm{mg}$, $40.5 \mu \mathrm{mol}$ ) and 2,3-dichloro-5,6-dicyano-p-benzoquinone (DDQ, $91.9 \mathrm{mg}, 405 \mu \mathrm{mol}$ ) in $\mathrm{CH}_{2} \mathrm{Cl}_{2}(4.05 \mathrm{~mL})$ was heated to $60^{\circ} \mathrm{C}$ in a sealed tube. After the reaction mixture was stirred at $60{ }^{\circ} \mathrm{C}$ for $40 \mathrm{~h}$, saturated aqueous $\mathrm{NaHCO}_{3}(5 \mathrm{~mL})$ and saturated aqueous $\mathrm{Na}_{2} \mathrm{~S}_{2} \mathrm{O}_{7}(5 \mathrm{~mL})$ were added to the mixture at $25^{\circ} \mathrm{C}$. The resultant mixture was extracted with EtOAc $(10 \mathrm{~mL}$ x5). The combined organic layers were washed with saturated aqueous $\mathrm{NaHCO}_{3}(30 \mathrm{~mL})$, dried over $\mathrm{Na}_{2} \mathrm{SO}_{4}$, and concentrated. The residue was purified by flash column chromatography on silica gel (Fuji Silysia, $28 \mathrm{~g}$, hexane/EtOAc $=2 / 1$ to $1 / 2$ ) to afford the crude alcohol 41 (36.9 mg, $32.5 \mu \mathrm{mol})$, which was used in the next reaction without further purification. HRMS (ESI-TOF) calcd for $\mathrm{C}_{59} \mathrm{H}_{52} \mathrm{~N}_{4} \mathrm{O}_{20} \mathrm{Na}[\mathrm{M}+\mathrm{Na}]^{+}$1159.3067, found 1159.3042 .

A solution of TMSOTf $\left(0.65 \mathrm{M}\right.$ in $\left.\mathrm{CH}_{2} \mathrm{Cl}_{2}, 200 \mu \mathrm{L}, 130 \mu \mathrm{mol}\right)$ was added to a solution of the above crude alcohol $41(36.9 \mathrm{mg}, 32.5 \mu \mathrm{mol})$ and 4 (108 mg, $163 \mu \mathrm{mol})$ in $\mathrm{CH}_{2} \mathrm{Cl}_{2}(900 \mu \mathrm{L})$ at $-20{ }^{\circ} \mathrm{C}$. The reaction mixture was gradually warmed to $0{ }^{\circ} \mathrm{C}$ and stirred for $16 \mathrm{~h}$, and then saturated aqueous $\mathrm{NaHCO}_{3}(2 \mathrm{~mL})$ was added. The resultant mixture was extracted with EtOAc $(3 \mathrm{~mL} \times 5)$. The combined organic layers were dried over $\mathrm{Na}_{2} \mathrm{SO}_{4}$ and concentrated. The residue was purified by PTLC $(10 \mathrm{~cm}$ x $20 \mathrm{~cm}, 4$ plates, hexane/EtOAc $=2 / 3)$ to afford the fully protected hikizimycin $42(26.1 \mathrm{mg}, 15.9 \mu \mathrm{mol})$ and 12-epi-42 $(14.3 \mathrm{mg}, 8.74 \mu \mathrm{mol})$ in 
$39 \%$ and 22\% yields, respectively, over 2 steps from 40.

42: colorless oil; $[\alpha]_{\mathrm{D}}{ }^{22}-17.0$ (c 1.23, $\mathrm{CHCl}_{3}$ ); IR (film) 3066, 2957, 2107, 1730, 1631, 1554, 1482, 1452, 1372, 1269, 1068, 1028, 802, $712 \mathrm{~cm}^{-1} ;{ }^{1} \mathrm{H}$ NMR (500 MHz, $\left.\mathrm{CO}\left(\mathrm{CD}_{3}\right)_{2}\right): \delta 1.74$ $\left(3 \mathrm{H}, \mathrm{s}, \mathrm{CH} \mathrm{H}_{3} \mathrm{CO}\right), 1.88\left(3 \mathrm{H}, \mathrm{s}, \mathrm{CH}_{3} \mathrm{CO}\right), 2.01\left(3 \mathrm{H}, \mathrm{s}, \mathrm{CH}_{3} \mathrm{CO}\right), 2.16\left(3 \mathrm{H}, \mathrm{s}, \mathrm{CH}_{3} \mathrm{CO}\right), 4.11-4.20$ (3H, m, H11a, H14, H15), 4.24 (1H, dd, $J=9.8,9.8$ Hz, H13), 4.32 (1H, d, $J=8.6$ Hz, H6), 4.50-4.55 (2H, m, H11b, H17a), 4.60-4.69 (2H, m, H16, H17b), 4.93-4.99 (2H, m, H4, H10), 5.11-5.17 (2H, m, H5, H7), 5.29 (1H, d, $J=9.8 \mathrm{~Hz}, \mathrm{H} 12), 5.38$ (1H, d, $J=9.2 \mathrm{~Hz}, \mathrm{H} 9), 5.74$ $(1 \mathrm{H}, \mathrm{d}, J=9.8 \mathrm{~Hz}, \mathrm{H} 8), 5.83$ (1H, brdd, $J=9.7,9.7 \mathrm{~Hz}, \mathrm{H} 2), 6.20(1 \mathrm{H}, \mathrm{dd}, J=9.7,9.7 \mathrm{~Hz}, \mathrm{H} 3)$, $6.53(1 \mathrm{H}, \mathrm{brd}, J=9.7 \mathrm{~Hz}, \mathrm{H1}), 7.33-7.41$ (4H, m, aromatic), 7.42-7.46 (2H, m, aromatic), 7.48$7.61(13 \mathrm{H}, \mathrm{m}$, aromatic, H19), 7.62-7.72 (5H, m, aromatic), 7.83-7.96 (7H, m, aromatic), 8.05$8.14\left(9 \mathrm{H}, \mathrm{m}\right.$, aromatic), $8.47(1 \mathrm{H}, \mathrm{d}, \mathrm{J}=7.5 \mathrm{~Hz}, \mathrm{H} 18)$; Detectable signals of ${ }^{13} \mathrm{C}$ NMR (125 $\left.\mathrm{MHz}, \mathrm{CO}\left(\mathrm{CD}_{3}\right)_{2}\right): \delta 20.6,20.9,21.1,21.2,54.0,63.1,64.4,64.7,68.2,68.4,68.6,68.7,70.8$, 72.1, 72.6, 73.1, 74.1, 76.3, 82.5, 98.1, 98.9, 124.1, 125.1, 129.2, 129.5, 129.7, 130.0, 130.38, $130.42,130.5,130.6,130.7,130.8,130.9,131.0,132.6,133.0,133.8,134.2,134.5,134.6,134.7$, $134.8,135.66,135.71,145.9,154.7,164.0,165.4,165.9,166.0,166.2,166.4,166.6,167.9$, 168.9, 170.48, 170.55, 171.0, 171.5; HRMS (ESI-TOF) calcd for $\mathrm{C}_{86} \mathrm{H}_{73} \mathrm{~N}_{7} \mathrm{O}_{27} \mathrm{Na}_{2}[\mathrm{M}+2 \mathrm{Na}]^{2+}$ 840.7169 , found 840.7194 .

12-epi-42: colorless oil; $[\alpha]_{\mathrm{D}}{ }^{22}+25.3\left(c 1.14, \mathrm{CHCl}_{3}\right.$ ); IR (film) 3066, 2958, 2110, 1729, 1484 , 1452, 1373, 1219, 1069, 801, $712 \mathrm{~cm}^{-1}$; ${ }^{1} \mathrm{H}$ NMR (500 MHz, $\left.\mathrm{CO}\left(\mathrm{CD}_{3}\right)_{2}\right): \delta 1.37(3 \mathrm{H}$, brs, $\left.\mathrm{CH}_{3} \mathrm{CO}\right), 1.77\left(3 \mathrm{H}, \mathrm{s}, \mathrm{CH}_{3} \mathrm{CO}\right), 2.06\left(3 \mathrm{H}, \mathrm{s}, \mathrm{CH}_{3} \mathrm{CO}\right), 2.24\left(3 \mathrm{H}, \mathrm{s}, \mathrm{CH}_{3} \mathrm{CO}\right), 3.91$ (1H, brs, H6), $4.08(1 \mathrm{H}, \mathrm{dd}, J=12.6,6.0 \mathrm{~Hz}, \mathrm{H} 11 \mathrm{a}), 4.38$ (1H, dd, $J=12.0,3.6 \mathrm{~Hz}, \mathrm{H} 17 \mathrm{a}), 4.43$ (1H, m, H16), $4.55(1 \mathrm{H}, \mathrm{dd}, J=12.6,2.9 \mathrm{~Hz}, \mathrm{H} 11 \mathrm{~b}), 4.61$ (1H, dd, $J=12.0,3.7 \mathrm{~Hz}, \mathrm{H} 17 \mathrm{~b}), 4.66$ (1H, dd, $J=$ 10.3, 10.3 Hz, H14), 5.06 (1H, m, H10), 5.15 (1H, dd, $J=10.3,3.5 \mathrm{~Hz}, \mathrm{H} 13), 5.29$ (1H, d, $J=$ $9.7 \mathrm{~Hz}, \mathrm{H} 9), 5.36$ (1H, dd, $J=10.3,10.3 \mathrm{~Hz}, \mathrm{H} 4), 5.42(1 \mathrm{H}, \mathrm{d}, J=9.7 \mathrm{~Hz}, \mathrm{H} 7), 5.51(1 \mathrm{H}, \mathrm{dd}$, $J=10.3,10.3 \mathrm{~Hz}, \mathrm{H} 15), 5.59$ (1H, d, $J=10.3 \mathrm{~Hz}, \mathrm{H} 5), 5.77$ (1H, brs, H2), 5.85 (1H, d, $J=9.7$ Hz, H8), $5.94(1 \mathrm{H}, \mathrm{d}, J=3.5 \mathrm{~Hz}, \mathrm{H} 12), 6.38(1 \mathrm{H}, \mathrm{dd}, J=10.3,10.3 \mathrm{~Hz}, \mathrm{H} 3), 6.51$ (1H, brd, $J$ $=8.1 \mathrm{~Hz}, \mathrm{H1}), 7.35-7.40(4 \mathrm{H}, \mathrm{m}$, aromatic, $\mathrm{H} 19), 7.42-7.60(15 \mathrm{H}, \mathrm{m}$, aromatic $), 7.62-7.70(5 \mathrm{H}$, $\mathrm{m}$, aromatic), 7.74-7.76 (2H, m, aromatic), 7.86-7.91 (4H, m, aromatic, H18), $7.95(1 \mathrm{H}, \mathrm{m}$, aromatic), 8.00-8.06 (4H, m, aromatic), 8.08-8.11 (4H, m, aromatic), 8.17-8.20 (2H, m, aromatic); Detectable signals of ${ }^{13} \mathrm{C}$ NMR $\left(125 \mathrm{MHz}, \mathrm{CO}\left(\mathrm{CD}_{3}\right)_{2}\right): \delta 20.1,21.1,21.20,21.24$, $51.5,62.30,63.34,64.5,67.3,67.9,68.7,68.9,69.3,70.8,71.8,72.6,73.5,74.3,82.9,93.5$, 98.6, 124.7, 124.9, 129.3, 129.4, 129.5, 129.6, 129.7, 130.0, 130.3, 130.5, 130.6, 130.72, 130.76, 130.82, 130.91, 132.0, 132.4, 133.9, 134.0, 134.3, 134.63, 134.70, 134.74, 136.1, 144.9, 154.3, $163.8,165.7,166.1,166.2,166.5,166.6,167.7,168.9,170.3,170.5,170.8,171.1$; HRMS (ESI- 
TOF) calcd for $\mathrm{C}_{86} \mathrm{H}_{73} \mathrm{~N}_{7} \mathrm{O}_{27} \mathrm{Na}_{2}[\mathrm{M}+2 \mathrm{Na}]^{2+} 840.7169$, found 840.7140 .
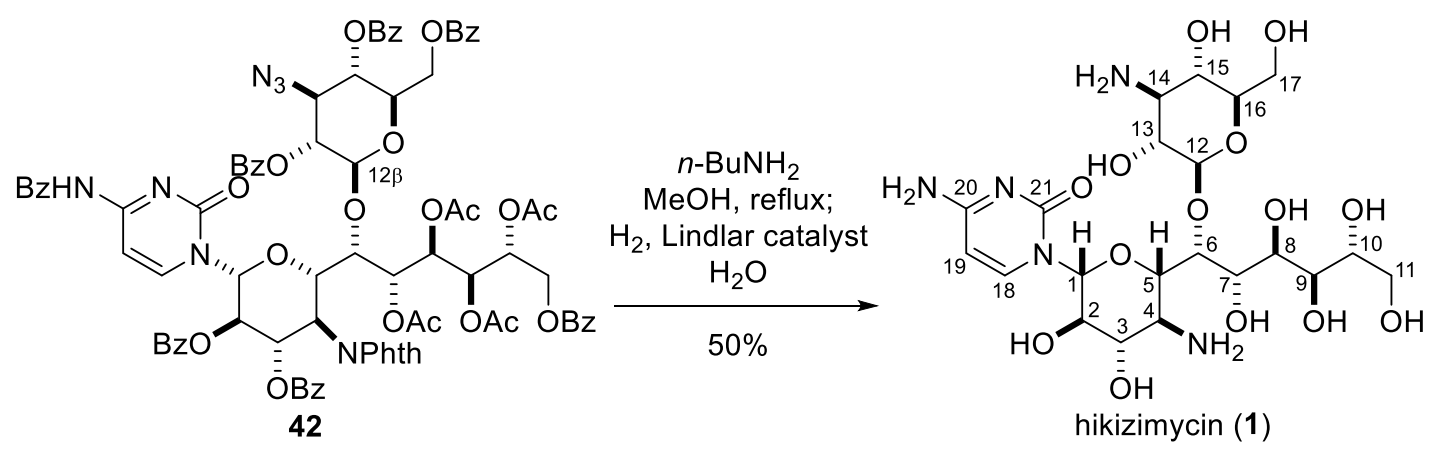

Hikizimycin (1) [12706-94-4]. ${ }^{\mathrm{S} 6} \quad$ A solution of the fully protected hikizimycin $42(33.5 \mathrm{mg}$, $20.5 \mu \mathrm{mol})$ in $n-\mathrm{BuNH}_{2}(2.56 \mathrm{~mL})$ and $\mathrm{MeOH}(2.56 \mathrm{~mL})$ was heated to reflux for $15 \mathrm{~h}$. After the reaction mixture was concentrated, Lindlar catalyst $(168 \mathrm{mg})$ and $\mathrm{H}_{2} \mathrm{O}(5.12 \mathrm{~mL})$ were added. The resultant mixture was stirred under $\mathrm{H}_{2}$ atmosphere at $25^{\circ} \mathrm{C}$ for $4 \mathrm{~h}$. Then, the mixture was filtered through membrane with $\mathrm{MeOH}(10 \mathrm{~mL})$ and then the filtrate was concentrated. The residue was purified by HPLC (column: Shodex NH2P-50 10E $10 \times 250$ $\mathrm{mm}$, eluent: $\mathrm{MeCN} / \mathrm{H}_{2} \mathrm{O}=75 / 25$ isocratic, flow rate: $3.0 \mathrm{~mL} / \mathrm{min}$, detection: UV $254 \mathrm{~nm}$ ) to afford hikizimycin $1(6.01 \mathrm{mg}, 10.3 \mu \mathrm{mol})$ in 50\% yield (Page S78): colorless solid; $[\alpha]_{\mathrm{D}}{ }^{26}$ 14.3 (c 0.43, $\mathrm{H}_{2} \mathrm{O}$ ); IR (film) 3350, 2925, 1657, 1606, 1498, 1384, 1292, 1205, $1076 \mathrm{~cm}^{-1}$; ${ }^{1} \mathrm{H}$ NMR (400 MHz, $\left.\mathrm{D}_{2} \mathrm{O}\right): \delta 2.84(1 \mathrm{H}, \mathrm{dd}, J=9.8,9.8 \mathrm{~Hz}, \mathrm{H} 14), 3.07(1 \mathrm{H}, \mathrm{dd}, J=9.4,9.4 \mathrm{~Hz}$, H4), 3.29 (1H, dd, $J=9.8,7.8 \mathrm{~Hz}, \mathrm{H} 13), 3.34$ (1H, dd, $J=9.8,9.8 \mathrm{~Hz}, \mathrm{H} 15), 3.49$ (1H, m, H16), 3.56 (1H, dd, $J=9.4,9.4$ Hz, H3), 3.66 (1H, dd, $J=11.9,6.2 \mathrm{~Hz}, \mathrm{H} 11 \mathrm{a}), 3.68-3.81$ (4H, m, H2, H9, H10, H17a), 3.86 (1H, dd, $J=11.9,2.5$ Hz, H11b), 3.90-3.95 (3H, m, H5, H7, H17b), $4.01(1 \mathrm{H}, \mathrm{d}, J=10.0 \mathrm{~Hz}, \mathrm{H} 8), 4.34(1 \mathrm{H}, \mathrm{d}, J=4.8 \mathrm{~Hz}, \mathrm{H} 6), 4.65(1 \mathrm{H}, \mathrm{d}, J=7.8 \mathrm{~Hz}$, H12), $5.58(1 \mathrm{H}, \mathrm{d}, J=9.6 \mathrm{~Hz}, \mathrm{H} 1), 6.08(1 \mathrm{H}, \mathrm{d}, J=7.5 \mathrm{~Hz}, \mathrm{H} 19), 7.70(1 \mathrm{H}, \mathrm{d}, J=7.5 \mathrm{~Hz}$, $\mathrm{H} 18) ;{ }^{13} \mathrm{C}$ NMR $\left(100 \mathrm{MHz}, \mathrm{D}_{2} \mathrm{O}\right) \delta 53.6,57.5,61.0,63.4,68.0,68.9,69.1,69.3,71.0,71.5$, 73.2, 76.7, 77.3, 78.6, 79.5, 84.0, 97.1, 104.2, 142.1, 158.1, 166.2; HRMS (ESI-TOF) calcd for $\mathrm{C}_{21} \mathrm{H}_{37} \mathrm{~N}_{7} \mathrm{O}_{14} \mathrm{Na}[\mathrm{M}+\mathrm{Na}]^{+}$606.2229, found 606.2203. 
2. Comparison of ${ }^{1} \mathrm{H}$ and ${ }^{13} \mathrm{C}$ NMR data of the reported and synthetic hikizimycin (1) in $\mathbf{D}_{2} \mathbf{O}$

Table S1. ${ }^{1} \mathrm{H}$ NMR data of reported ${ }^{\mathrm{S} 12}$ and synthetic hikizimycin $(\mathbf{1})$ in $\mathrm{D}_{2} \mathrm{O}$

\begin{tabular}{rll}
\hline & reported 1 $(500 \mathrm{MHz})$ & synthetic $\mathbf{1}(400 \mathrm{MHz})$ \\
\cline { 2 - 3 } No. & ${ }^{1} \mathrm{H}[\delta$, multi, $J(\mathrm{~Hz})]$ & ${ }^{1} \mathrm{H}[\delta$, multi, $J(\mathrm{~Hz})]$ \\
\hline 1 & $5.43(\mathrm{~d}, 9.4)$ & $5.58(\mathrm{~d}, 9.6)$ \\
3 & $3.61(\mathrm{~m})$ & $3.68-3.81(\mathrm{~m})$ \\
4 & $3.40(\mathrm{dd}, 9.3,9.3)$ & $3.56(\mathrm{dd}, 9.4,9.4)$ \\
5 & $2.90(\mathrm{dd}, 9.8,9.8)$ & $3.07(\mathrm{dd}, 9.4,9.4)$ \\
6 & $3.77(\mathrm{~m})$ & $3.90-3.95(\mathrm{~m})$ \\
7 & $3.77(\mathrm{~m})$ & $4.34(\mathrm{~d}, 4.8)$ \\
8 & $3.86(\mathrm{~d}, 9.8)$ & $3.90-3.95(\mathrm{~m})$ \\
9 & $3.61(\mathrm{~m})$ & $4.01(\mathrm{~d}, 10.0)$ \\
10 & $3.61(\mathrm{~m})$ & $3.68-3.81(\mathrm{~m})$ \\
$11 \mathrm{a}$ & $3.52(\mathrm{dd}, 11.7,6.2)$ & $3.68-3.81(\mathrm{~m})$ \\
$11 \mathrm{~b}$ & $3.71(\mathrm{dd}, 11.7,2.6)$ & $3.66(\mathrm{dd}, 11.9,6.2)$ \\
12 & $4.49(\mathrm{~d}, 7.8)$ & $3.85(\mathrm{dd}, 11.9,2.5)$ \\
13 & $3.12(\mathrm{dd}, 10.0,7.8)$ & $4.65(\mathrm{~d}, 7.8)$ \\
14 & $2.65(\mathrm{dd}, 9.8,9.8)$ & $3.29(\mathrm{dd}, 9.8,7.8)$ \\
15 & $3.16(\mathrm{dd}, 9.7,9.7)$ & $2.84(\mathrm{dd}, 9.8,9.8)$ \\
16 & $3.33(\mathrm{~m})$ & $3.34(\mathrm{dd}, 9.8,9.8)$ \\
$17 \mathrm{a}$ & $3.61(\mathrm{~m})$ & $3.49(\mathrm{~m})$ \\
$17 \mathrm{~b}$ & $3.77(\mathrm{~m})$ & $3.68-3.81(\mathrm{~m})$ \\
18 & $7.56(\mathrm{~d}, 7.6)$ & $3.90-3.95(\mathrm{~m})$ \\
19 & $5.94(\mathrm{~d}, 7.5)$ & $7.70(\mathrm{~d}, 7.5)$ \\
& & $6.08(\mathrm{~d}, 7.5)$ \\
\hline &
\end{tabular}


Table S2. ${ }^{13} \mathrm{C}$ NMR data of reported ${ }^{\mathrm{S} 12}$ and synthetic hikizimycin (1) in $\mathrm{D}_{2} \mathrm{O}$

\begin{tabular}{cc}
\hline reported $\mathbf{1}(100 \mathrm{MHz})$ & synthetic $\mathbf{1}(100 \mathrm{MHz})$ \\
\hline${ }^{13} \mathrm{C}(\delta)$ & ${ }^{13} \mathrm{C}(\delta)$ \\
\hline 53.14 & 53.61 \\
57.14 & 57.55 \\
60.60 & 60.95 \\
63.02 & 63.45 \\
67.59 & 67.97 \\
68.61 & 68.95 \\
68.67 & 69.07 \\
69.09 & 69.27 \\
70.60 & 70.99 \\
71.02 & 71.46 \\
72.99 & 73.20 \\
76.49 & 76.75 \\
76.90 & 77.31 \\
78.21 & 78.63 \\
79.41 & 79.53 \\
83.59 & 84.00 \\
96.62 & 97.10 \\
103.84 & 104.23 \\
141.70 & 142.11 \\
157.66 & 158.13 \\
165.73 & 166.19
\end{tabular}



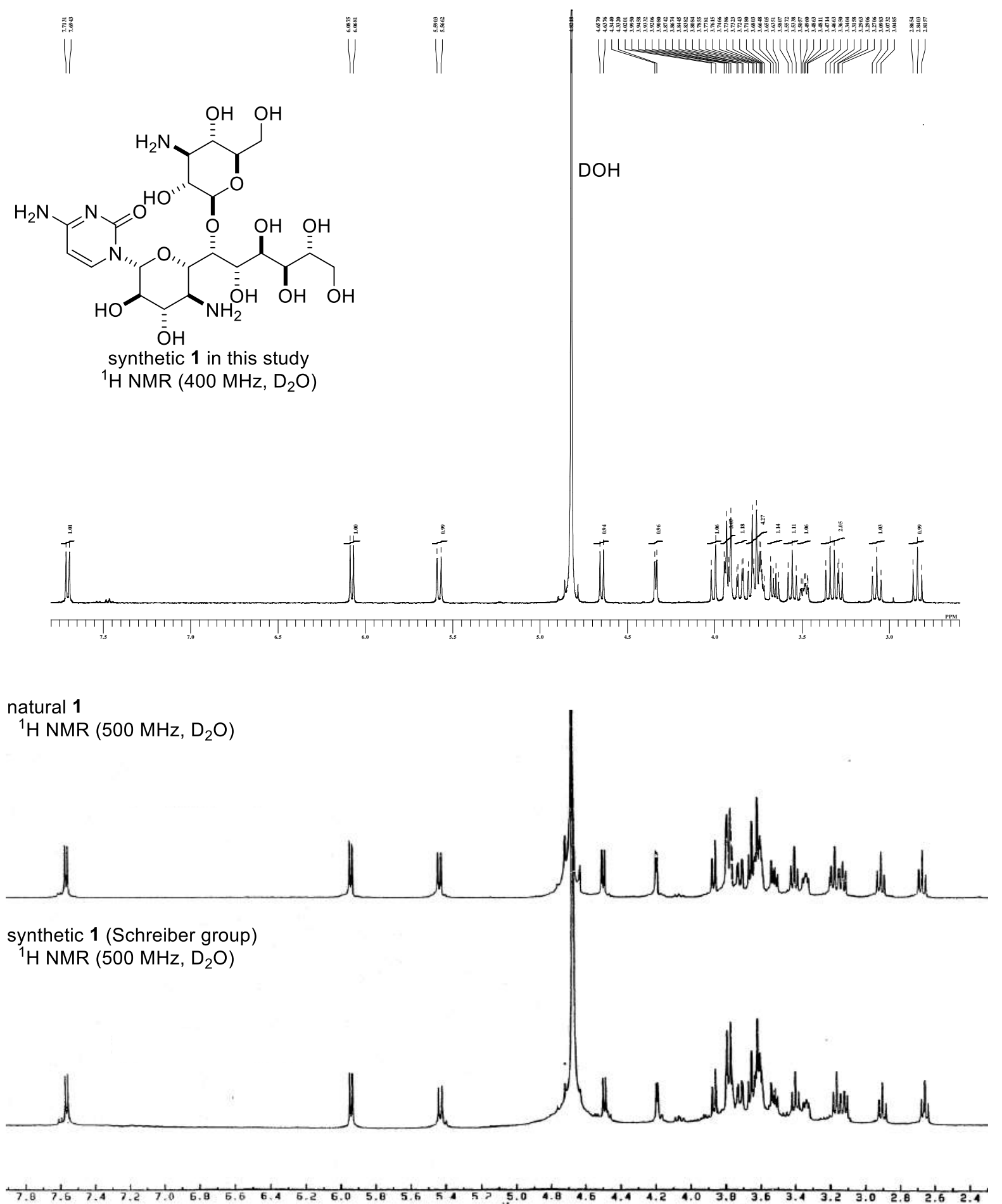

Figure S2. ${ }^{1} \mathrm{H}$ NMR spectra of synthetic 1 in this study (400 MHz), natural $1(500 \mathrm{MHz})$, and synthetic 1 (Schreiber group, $500 \mathrm{MHz}$ ) 
<smiles>Nc1ccn([C@@H]2O[C@H](OC3OC(CO)[C@@H](O)[C@H](N)[C@H]3O)[C@H](O)C(O)[C@H]2N)c(=O)n1</smiles>

synthetic 1 in this study

${ }^{13} \mathrm{C}$ NMR $\left(100 \mathrm{MHz}, \mathrm{D}_{2} \mathrm{O}\right)$

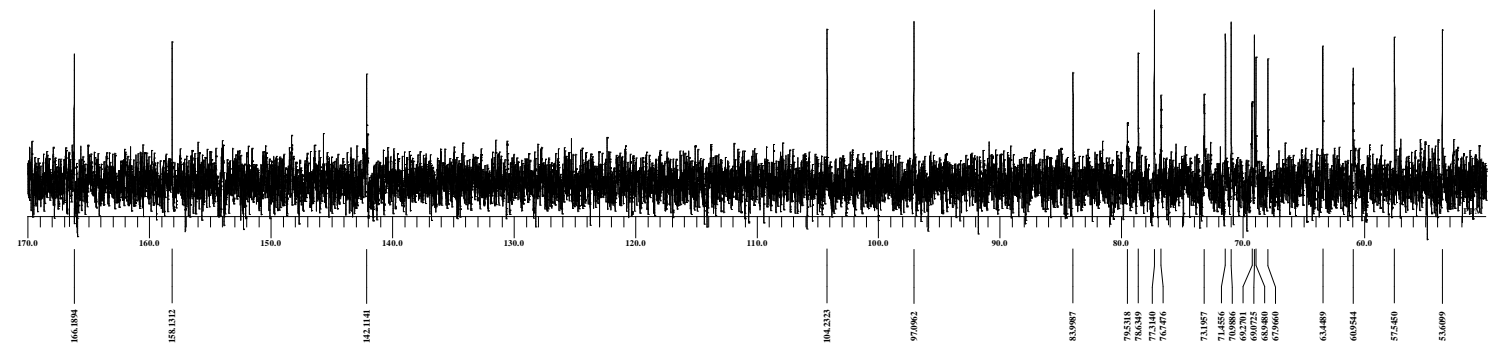

natural 1

${ }^{13} \mathrm{C}$ NMR $\left(100 \mathrm{MHz}, \mathrm{D}_{2} \mathrm{O}\right)$
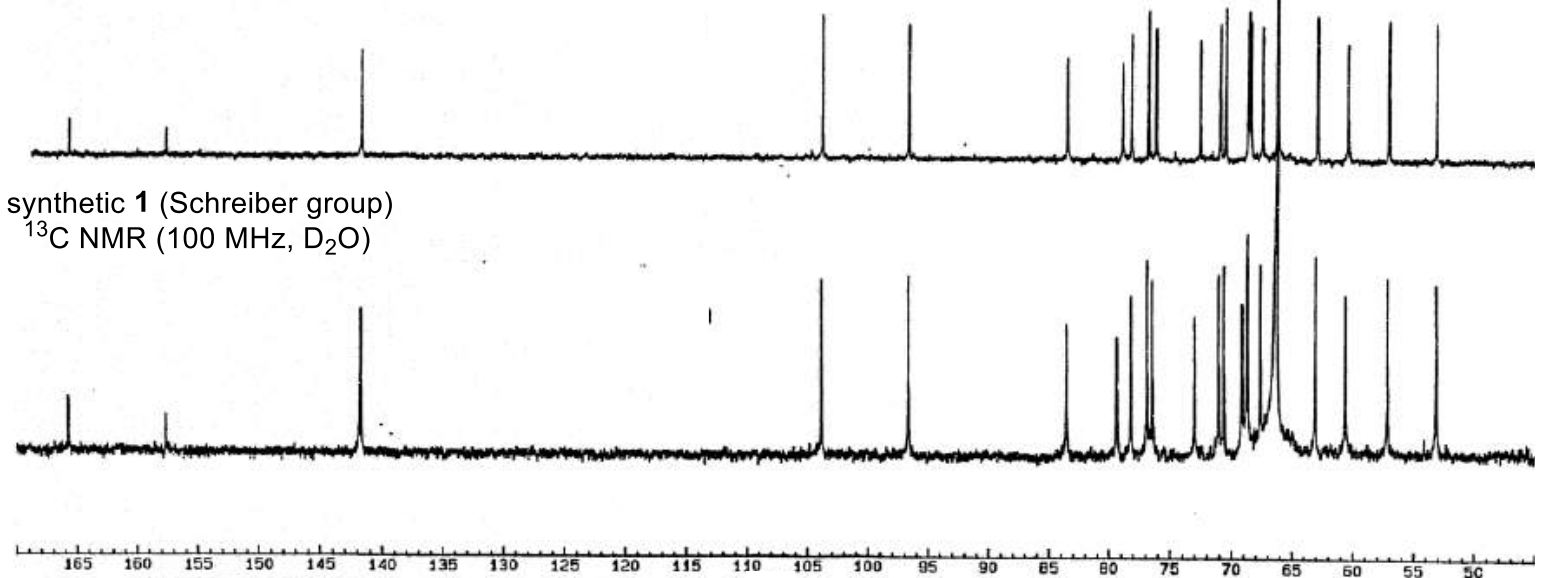

Figure S3. ${ }^{13} \mathrm{C}$ NMR spectra of synthetic 1 in this study $(100 \mathrm{MHz})$, natural $\mathbf{1}(100 \mathrm{MHz})$, and synthetic 1 (Schreiber group, $100 \mathrm{MHz}$ ) 


\section{Investigation of the effect of $\mathrm{C} 4-\mathrm{N}$-protective group and $\mathrm{C} 1$-stereochemistry on the radical addition reactions}

Prior to the key radical coupling reaction, we performed the model experiments using four $\alpha$ alkoxyacyl tellurides $\mathbf{S 9 a - d}$ as radical precursors and methyl vinyl ketone as a radical acceptor (Table S3). First, the N-phthaloyl group (entry 3) was found to be superior to the Ntrifluoroacetaamide (entry 1) or N-2,3-dimethylmaleimide (entry 2) at C4. When trifluoroacetamide S9a was used (entry 1), only the undesired C5 $\beta$-stereoisomer S10a- $\boldsymbol{\beta}$ was obtained (entry 1). In contrast, when the imide groups were employed (entries 2 and 3), the desired C5 $\alpha$-stereoisomer S10b/c- $\alpha$ was exclusively formed, and S10c- $\alpha$ with the N-phthaloyl group was generated in the higher yield (76\%). Second, the C1 $\alpha$-stereochemistry (entry 3) was revealed to be more beneficial for the radical addition than the $\mathrm{C} 1 \beta$-counterpart (entry 4 ). Decarbonylation from S9d with the C1 $\beta$-methoxy group (entry 4) was slower than that from S9c (entry 3). Consequently, the yield of the adduct S10d- $\alpha$ (25\%) was lower than that of S10c- $\alpha$ (76\%) because S11d was produced through the direct addition of the acyl radical. Based on these results, 8 was designed to have the $\mathrm{C} 1 \alpha-\mathrm{O}$ and $\mathrm{C} 4 \mathrm{~N}-\mathrm{Phth}$ groups. 
Table S3. Investigation of the effect of C4-N-protective group and C1-stereochemistry on the radical addition reactions ${ }^{a}$
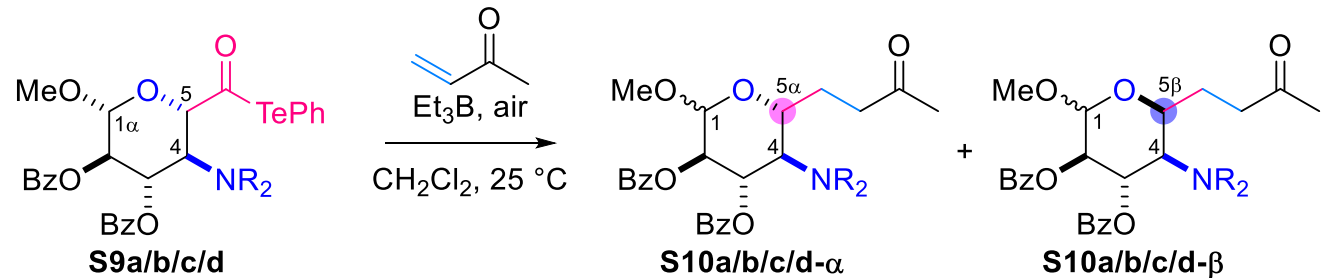

$\mathrm{S} 10 \mathrm{a} / \mathrm{b} / \mathrm{c} / \mathrm{d}-\alpha$

$\mathrm{S} 10 \mathrm{a} / \mathrm{b} / \mathrm{c} / \mathrm{d}-\beta$

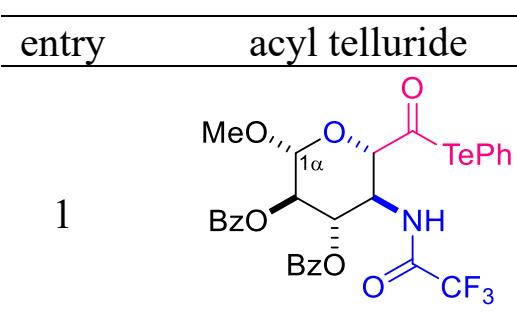

S9a

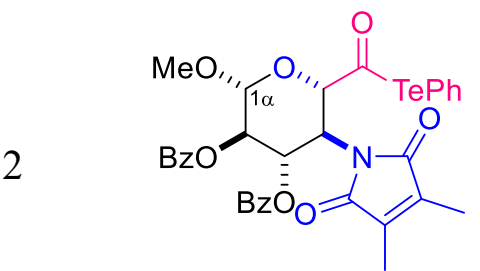

S9b

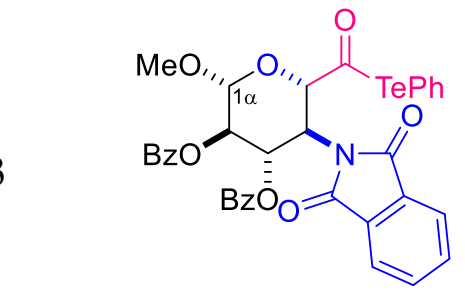

S9c

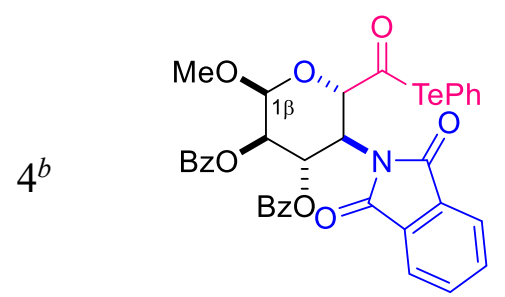

S9d<smiles>[2H][C@@H]1[C@@H](OC(=O)c2ccccc2)[C@@H](OC)O[C@H](CCC(C)=O)[C@@H]1NC(=O)C(F)(F)F</smiles>

S10a- $\alpha(0 \%)$<smiles>COC(=O)c1ccccc1</smiles>

S10b- $\alpha(54 \%)$

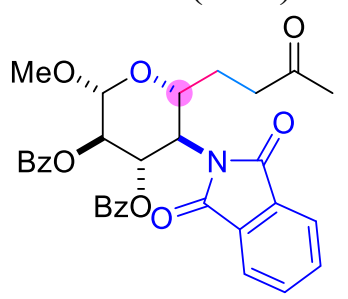

S10c- $\alpha(76 \%)$

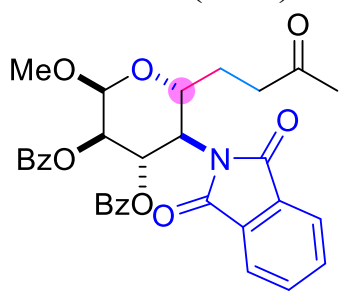

S10d- $\alpha(25 \%)$ results

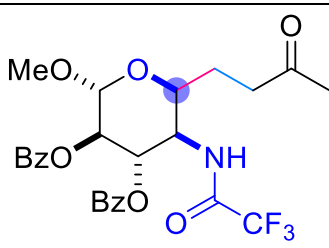

S10a- $\boldsymbol{\beta}(83 \%)$

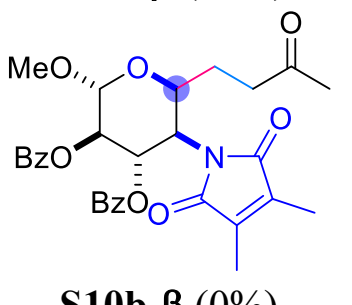<smiles>[AsH2][SeH][SeH]</smiles><smiles>COC(=O)c1ccccc1C(=O)OC(C)C(=O)OC(=O)c1ccccc1</smiles>

S10c- $\beta$ (0\%)<smiles>COC(=O)c1ccccc1C(=O)OC1C(OC)O[C@H](C(=O)CCC(C)=O)C(N2C(=O)c3ccccc3C2=O)C1C(=O)O</smiles>

S11d (38\%)

${ }^{a}$ Conditions: S9a-d (1 equiv), methyl vinyl ketone (2 equiv), $\mathrm{Et}_{3} \mathrm{~B}$ (3 equiv), $\mathrm{CH}_{2} \mathrm{Cl}_{2}(0.02 \mathrm{M})$, open air, $25{ }^{\circ} \mathrm{C}$.

${ }^{b}$ Reaction was conducted with $\mathrm{C} 1 \beta$ isomer and methyl vinyl ketone (2 equiv). 
4. X-ray structure and crystallographic data of 28- $\alpha$

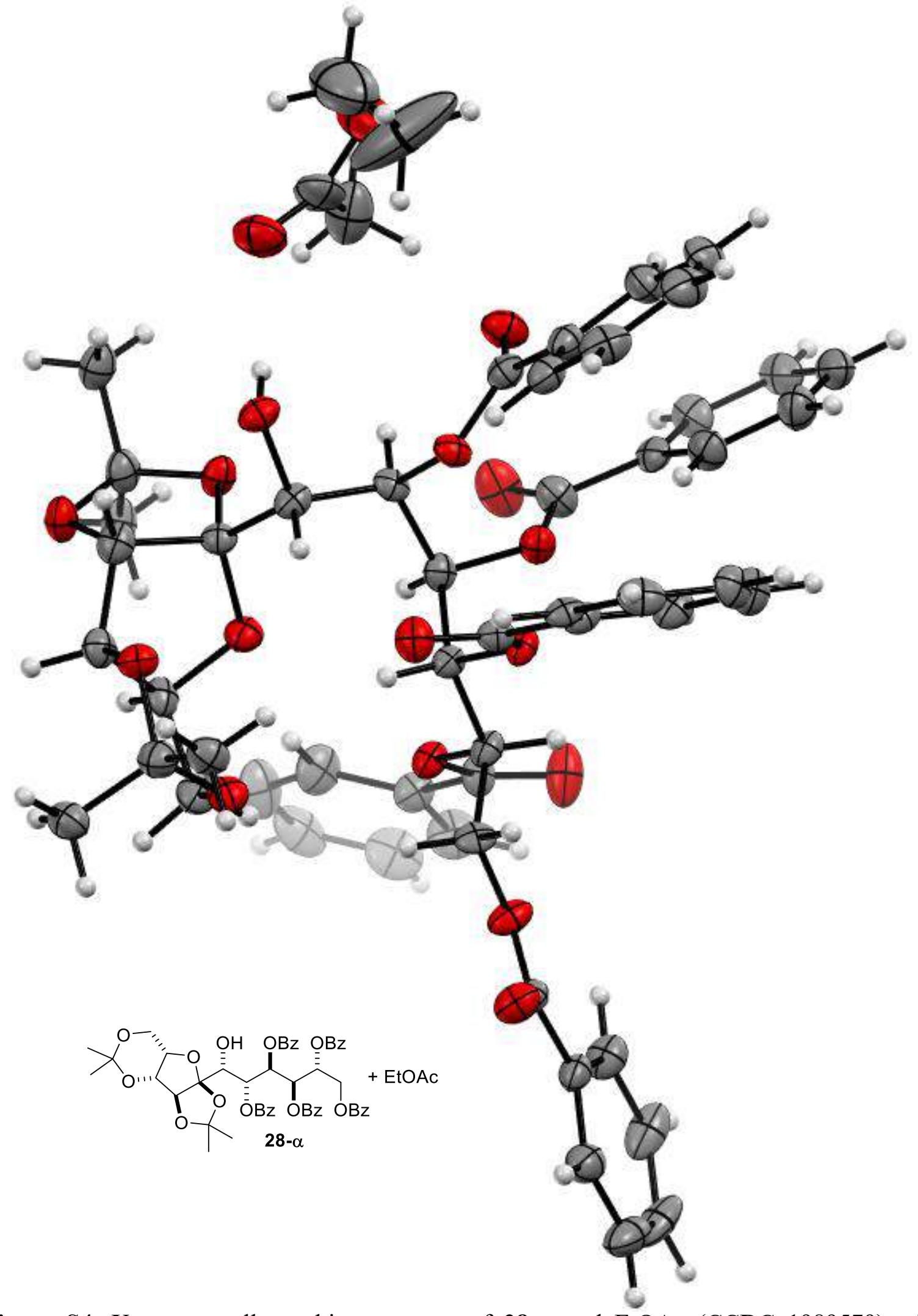

Figure S4. X-ray crystallographic structures of 28- $\alpha$ and EtOAc (CCDC 1989579) with thermal ellipsoids at the $50 \%$ probability level (ORTEP) 
Table S4. Crystal data and structure refinement for 28- $\alpha$

\begin{tabular}{|c|c|}
\hline Compound & $28-\alpha \cdot$ EtOAc \\
\hline CCDC number & 1989579 \\
\hline Molecular formula & $\mathrm{C}_{56} \mathrm{H}_{58} \mathrm{O}_{18}\left(\mathrm{C}_{52} \mathrm{H}_{50} \mathrm{O}_{16}+\mathrm{C}_{4} \mathrm{H}_{8} \mathrm{O}_{2}\right)$ \\
\hline Formula weight & $1019.062(930.956+88.106)$ \\
\hline Temperature (K) & $93(2)$ \\
\hline Wavelength $(\AA)$ & 0.71075 \\
\hline Crystal color, habit & colorless, prism \\
\hline Crystal size $\left(\mathrm{mm}^{3}\right)$ & $0.20 \times 0.20 \times 0.20$ \\
\hline Crystal system & monoclinic \\
\hline Space group & $\mathrm{P} 2{ }_{1}(\# 4)$ \\
\hline \multicolumn{2}{|l|}{ Unit cell dimensions } \\
\hline $\mathrm{a}(\AA)$ & $10.626(11)$ \\
\hline $\mathrm{b}(\AA)$ & $19.78(2)$ \\
\hline$c(\AA)$ & $12.823(14)$ \\
\hline$\alpha\left(^{\circ}\right)$ & 90.0000 \\
\hline$\beta\left(^{\circ}\right)$ & $99.803(11)$ \\
\hline$\gamma\left(\left(^{\circ}\right)\right.$ & 90.0000 \\
\hline Volume $\left(\AA^{3}\right)$ & $2656(5)$ \\
\hline$Z$ & 2 \\
\hline Density (calculated) $\left(\mathrm{g} / \mathrm{cm}^{3}\right)$ & 1.274 \\
\hline$\mu(\operatorname{MoK} \alpha)\left(\mathrm{cm}^{-1}\right)$ & 0.951 \\
\hline $\mathrm{F}(000)$ & 1076.00 \\
\hline \multirow[t]{3}{*}{ Index ranges } & $-13<=\mathrm{h}<12$ \\
\hline & $-25<=\mathrm{k}<17$ \\
\hline & $-16<=1<16$ \\
\hline Reflections collected & 19334 \\
\hline Independent reflections & 8965 \\
\hline $\mathrm{R}($ int $)$ & 0.0727 \\
\hline Completeness to theta & $25.242^{\circ}, 99.3 \%$ \\
\hline Max. and min. transmission & $0.698,0.981$ \\
\hline \multirow[t]{2}{*}{ Refinement method } & Full-matrix \\
\hline & least-squares on $\mathrm{F}^{2}$ \\
\hline No. Observations (All reflections) & 8965 \\
\hline No. Variables & 675 \\
\hline Reflection/Parameter Ratio & 13.28 \\
\hline Goodness-of-fit on $\mathrm{F}^{2}$ & 1.060 \\
\hline Residuals: R1 $(I>2.00 \sigma(I))$ & 0.0764 \\
\hline Residuals: R (All reflections) & 0.0927 \\
\hline Residuals: wR2 (All reflections) & 0.1639 \\
\hline Max. and min. peak in Final Diff. Map (e-/ $\left./ \AA^{3}\right)$ & $0.30,-0.29$ \\
\hline
\end{tabular}




\section{Structural determination of 29- $\alpha$}

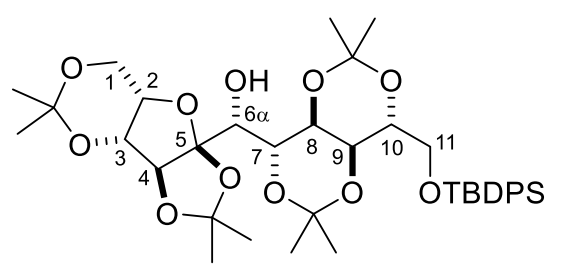

29- $\alpha$

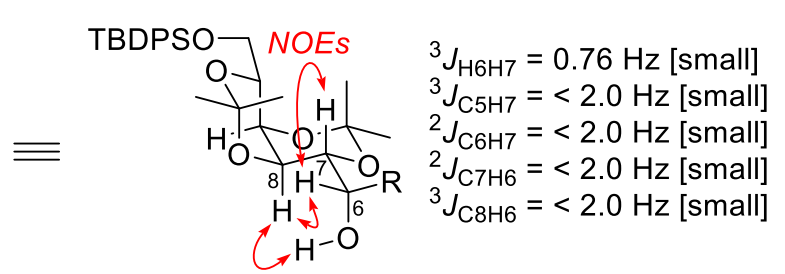

J-Based Configuration Analysis (JBCA methods)

Figure S5. Determination of the C6-stereochemistry of alcohol 29- $\alpha$ by $J$-Based Configuration Analysis (JBCA) in $\mathrm{C}_{6} \mathrm{D}_{6}$.

The C6-configuration of alcohol 29- $\alpha$ was determined by $J$-Based Configuration Analysis (JBCA). ${ }^{\mathrm{S} 13}$ The one interproton spin-coupling constant $\left({ }^{3} J_{\mathrm{H} 6 \mathrm{H} 7}: 0.76 \mathrm{~Hz}\right)$ and the four carbonproton spin-coupling constants $\left({ }^{3} J_{\mathrm{C} 5 \mathrm{H} 7}:<2.0 \mathrm{~Hz},{ }^{2} J_{\mathrm{C} 6 \mathrm{H} 7}:<2.0 \mathrm{~Hz},{ }^{2} J_{\mathrm{C} 7 \mathrm{H} 6}:<2.0 \mathrm{~Hz},{ }^{3} J_{\mathrm{C} 8 \mathrm{H} 6}:<\right.$ $2.0 \mathrm{~Hz}$ ) were obtained by analyses of DQF-COSY (Page S56) and $J$-resolved HMBC-1 (Page S58) NMR experiments in $\mathrm{C}_{6} \mathrm{D}_{6}$, respectively. These data indicated the above C6-C11 conformation. This assignment was further confirmed by NOESY data (Page S59) in $\mathrm{C}_{6} \mathrm{D}_{6}$ and the key NOE correlations are shown in red. Consequently, the C6 $\alpha$-stereochemistry of 29- $\alpha$ was determined.

\section{Structural determination of 30- $\alpha$}

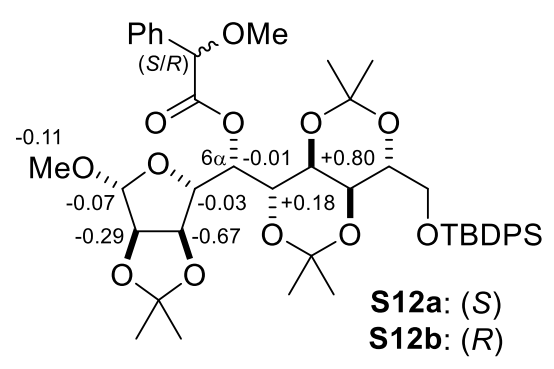

Figure S6. Determination of the C6-stereochemistry of alcohol 30- $\alpha$. The values are the differences $(\Delta \delta)$ in ${ }^{1} H$ chemical shifts between S12b and S12a $(\Delta \delta=\delta(\mathbf{S 1 2 b})-\delta(\mathbf{S 1 2 a}))$ in $\mathrm{CDCl}_{3}$.

The C6-configuration of alcohol 30- $\alpha$ was determined by application of the modified Mosher method (Figure S6). ${ }^{\text {S14 }}$ Namely, 30- $\boldsymbol{\alpha}$ were derivatized into $(S)$-MPA ester S12a and $(R)$-MPA ester S12b. The difference in ${ }^{1} \mathrm{H}$ NMR chemical shifts between S12a and S12b were calculated to confirm the C6 $\alpha$-stereochemistry of $30-\alpha$. 

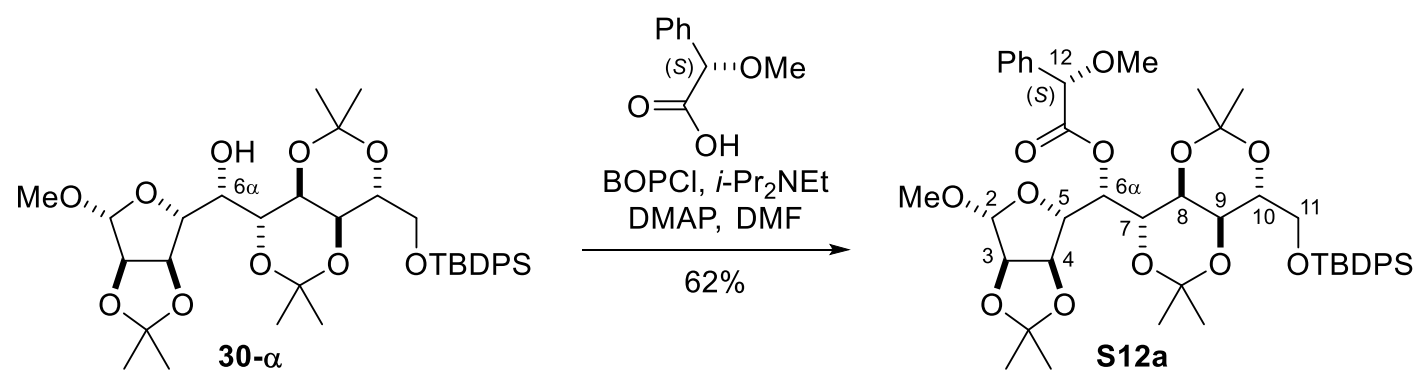

(S)-MPA ester S12a. Bis(2-oxo-3-oxazolidinyl)phosphorodiamidic chloride (BOPCl, 68.7 $\mathrm{mg}, 270 \mu \mathrm{mol})$ was added to a solution of DMAP (4.4 mg, $36 \mu \mathrm{mol}), i$-Pr ${ }_{2} \mathrm{NEt}(78.4 \mathrm{~mL}, 450$ $\mu \mathrm{mol}),(S)-\mathrm{MPA}(44.9 \mathrm{mg}, 270 \mu \mathrm{mol})$, and alcohol 30- $\alpha(12.1 \mathrm{mg}, 18.0 \mu \mathrm{mol})$ in DMF (900 $\mu \mathrm{L})$ at $25^{\circ} \mathrm{C}$. After being refluxed for $17 \mathrm{~h}$, saturated aqueous $\mathrm{NaHCO}_{3}(4 \mathrm{~mL})$ was added. Then, the resultant mixture was extracted with $\mathrm{Et}_{2} \mathrm{O}(4 \mathrm{~mL} \times 3)$. The combined organic layers were washed with brine $(15 \mathrm{~mL})$, dried over $\mathrm{Na}_{2} \mathrm{SO}_{4}$, filtered, and concentrated. The residue was filtered through a pad of silica gel $(15 \mathrm{~g}$, hexane/EtOAc $=1 / 1)$. The filtrate was concentrated and the residue was purified by PTLC $(10 \mathrm{~cm}$ x $20 \mathrm{~cm}, 2$ plates, hexane/ EtOAc $=4 / 1)$ to afford $(S)$-MPA ester S12a $(9.20 \mathrm{mg}, 11.2 \mu \mathrm{mol})$ in $62 \%$ yield: yellow oil; $[\alpha]_{\mathrm{D}}{ }^{27}=$ $+10.1\left(\right.$ c 0.46, $\mathrm{CHCl}_{3}$ ); IR (film) 2932, 1757, 1377, 1215, 1165, 1107, 872, $703 \mathrm{~cm}^{-1} ;{ }^{1} \mathrm{H}$ NMR $\left(500 \mathrm{MHz}, \mathrm{CDCl}_{3}\right) \delta 1.01\left(3 \mathrm{H}, \mathrm{s}, \mathrm{CH}_{3}\right.$ of acetonide), $1.04\left(9 \mathrm{H}, \mathrm{s}, \mathrm{C}\left(\mathrm{CH}_{3}\right)_{3}\right.$ of TBDPS), 1.12 ( $3 \mathrm{H}, \mathrm{s}, \mathrm{CH}_{3}$ of acetonide), $1.24\left(3 \mathrm{H}, \mathrm{s}, \mathrm{CH}_{3}\right.$ of acetonide), $1.29\left(3 \mathrm{H}, \mathrm{s}, \mathrm{CH}_{3}\right.$ of acetonide), 1.31 (3H, s, $\mathrm{CH}_{3}$ of acetonide), $1.48\left(3 \mathrm{H}, \mathrm{s}, \mathrm{CH}_{3}\right.$ of acetonide), $2.69(1 \mathrm{H}, \mathrm{dd}, J=9.5,4.6 \mathrm{~Hz}, \mathrm{H} 8)$, $2.99(1 \mathrm{H}, \mathrm{dd}, J=8.0,5.7 \mathrm{~Hz}, \mathrm{H} 10), 3.32\left(3 \mathrm{H}, \mathrm{s}, \mathrm{OCH}_{3}\right), 3.41\left(3 \mathrm{H}, \mathrm{s}, \mathrm{OCH}_{3}\right), 3.56-3.60(2 \mathrm{H}, \mathrm{m}$, H9, H11a), 3.65 (1H, dd, $J=14.0,6.0 \mathrm{~Hz}, \mathrm{H} 11 \mathrm{~b}), 3.77$ (1H, d, $J=9.5 \mathrm{~Hz}, \mathrm{H} 7), 4.35$ (1H, d, $J$ $=9.2 \mathrm{~Hz}, \mathrm{H} 5), 4.59(1 \mathrm{H}, \mathrm{d}, J=6.0 \mathrm{~Hz}, \mathrm{H} 4), 4.64(1 \mathrm{H}, \mathrm{d}, J=6.0 \mathrm{~Hz}, \mathrm{H} 3), 4.86(1 \mathrm{H}, \mathrm{s}, \mathrm{H} 12)$, $4.96(1 \mathrm{H}, \mathrm{s}, \mathrm{H} 2), 5.09(1 \mathrm{H}, \mathrm{d}, J=9.2 \mathrm{~Hz}, \mathrm{H} 6), 7.06(1 \mathrm{H}, \mathrm{m}$, aromatic), 7.21-7.26 (2H, m, aromatic), 7.36-7.47 (8H, m, aromatic), 7.68-7.73 (4H, m, aromatic); ${ }^{13} \mathrm{C}$ NMR (125 MHz, $\left.\mathrm{CDCl}_{3}\right), \delta 19.3,23.5,23.6,24.4,24.6,24.9,26.4,26.7$ (3C), 55.6, 57.0, 64.5, 66.5, 67.8, 67.9, $70.6,71.5,81.7,82.0,83.8,85.3,100.4,100.8,109.8,112.2,127.2(2 \mathrm{C}), 127.46(2 \mathrm{C}), 127.51$ (2C), 128.6 (2C), 128.8, 129.52, 129.55, 133.6, 133.9, 135.7 (2C), 135.9 (2C), 136.3, 170.0; HRMS (ESI-TOF) calcd for $\mathrm{C}_{45} \mathrm{H}_{60} \mathrm{O}_{12} \mathrm{SiNa}[\mathrm{M}+\mathrm{Na}]^{+}$843.3746, found 843.3730. 

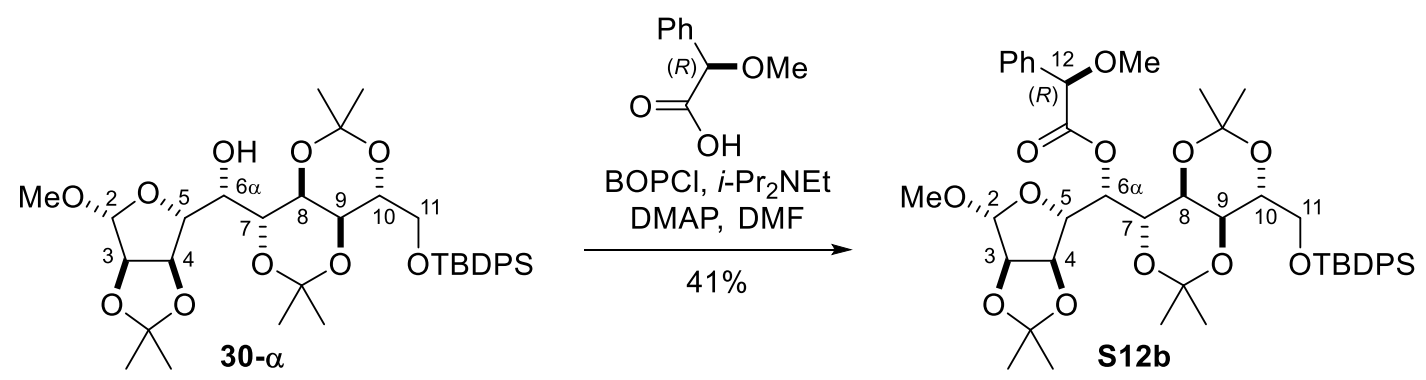

(R)-MPA ester S12b. $\quad$ BOPCl $(72.5 \mathrm{mg}, 285 \mu \mathrm{mol})$ was added to a solution of DMAP (4.6 mg, $38 \mu \mathrm{mol}$ ), $i$-Pr 2 NEt (82.7 mL, $475 \mu \mathrm{mol}),(R)$-MPA (47.4 mg, $285 \mu \mathrm{mol})$, and alcohol 30$\alpha(12.8 \mathrm{mg}, 19.0 \mu \mathrm{mol})$ in DMF $(951 \mu \mathrm{L})$ at $25^{\circ} \mathrm{C}$. After being refluxed for $17 \mathrm{~h}$, saturated aqueous $\mathrm{NaHCO}_{3}(4 \mathrm{~mL})$ was added. Then, the resultant mixture was extracted with $\mathrm{Et}_{2} \mathrm{O}(4$ $\mathrm{mL} \times 3)$. The combined organic layers were washed with brine $(15 \mathrm{~mL})$, dried over $\mathrm{Na}_{2} \mathrm{SO}_{4}$, filtered, and concentrated. The residue was filtered through a pad of silica gel $(15 \mathrm{~g}$, hexane/EtOAc $=1 / 1)$. The filtrate was concentrated and the residue was purified by PTLC $(10 \mathrm{~cm}$ x $20 \mathrm{~cm}, 2$ plates, hexane/ EtOAc = 4/1) to afford $(R)$-MPA ester S12b $(6.32 \mathrm{mg}, 7.70$ $\mu \mathrm{mol})$ in $41 \%$ yield: yellow oil; $[\alpha]_{\mathrm{D}}^{28}=-42.9\left(c 0.32, \mathrm{CHCl}_{3}\right)$; IR (film) 2932, 1748, 1458, 1377, 1216, 1164, 1105, $874 \mathrm{~cm}^{-1}$; ${ }^{1} \mathrm{H}$ NMR $\left(500 \mathrm{MHz}, \mathrm{CDCl}_{3}\right) \delta 1.04\left(9 \mathrm{H}, \mathrm{s}, \mathrm{C}\left(\mathrm{CH}_{3}\right)_{3}\right.$ of TBDPS), $1.08\left(3 \mathrm{H}, \mathrm{s}, \mathrm{CH}_{3}\right.$ of acetonide), $1.17\left(3 \mathrm{H}, \mathrm{s}, \mathrm{CH}_{3}\right.$ of acetonide), $1.28\left(3 \mathrm{H}, \mathrm{s}, \mathrm{CH}_{3}\right.$ of acetonide), $1.31\left(3 \mathrm{H}, \mathrm{s}, \mathrm{CH}_{3}\right.$ of acetonide), $1.38\left(3 \mathrm{H}, \mathrm{s}, \mathrm{CH}_{3}\right.$ of acetonide), $1.41\left(3 \mathrm{H}, \mathrm{s}, \mathrm{CH}_{3}\right.$ of acetonide), $3.31\left(3 \mathrm{H}, \mathrm{s}, \mathrm{OCH}_{3}\right), 3.43\left(3 \mathrm{H}, \mathrm{s}, \mathrm{OCH}_{3}\right), 3.49(1 \mathrm{H}, \mathrm{dd}, J=9.2,4.6 \mathrm{~Hz}, \mathrm{H} 8), 3.66-$ 3.79 (4H, m, H9, H10, H11), $3.92(1 \mathrm{H}, \mathrm{d}, J=6.3 \mathrm{~Hz}, \mathrm{H} 4), 3.95(1 \mathrm{H}, \mathrm{d}, J=9.2 \mathrm{~Hz}, \mathrm{H} 7), 4.32$ $(1 \mathrm{H}, \mathrm{d}, J=10.3 \mathrm{~Hz}, \mathrm{H} 5), 4.35$ (1H, d, $J=6.3 \mathrm{~Hz}, \mathrm{H} 3), 4.87$ (1H, s, H12), 4.89 (1H, s, H2), 5.08 $(1 \mathrm{H}, \mathrm{d}, J=10.3 \mathrm{~Hz}, \mathrm{H} 6), 7.23$ (1H, m, aromatic), 7.30-7.44 (8H, m, aromatic), 7.49-7.52 (2H, $\mathrm{m}$, aromatic), 7.70-7.73 (4H, m, aromatic); $\left.{ }^{13} \mathrm{C} \mathrm{NMR} \mathrm{(125} \mathrm{MHz,} \mathrm{CDCl}_{3}\right), \delta 19.3,23.6,23.7$, 24.48, 24.53, 24.7, 26.2, 26.7 (3C), 55.5, 57.3, 64.4, 67.1, 67.8, 68.0, 70.8, 71.4, 81.3, 82.7, 83.1, 84.9, 100.6, 101.3, 109.7, 111.9, 127.2 (2C), 127.48 (2C), 127.51 (2C), $128.6(2 \mathrm{C}), 128.9$, 129.5 (2C), 133.7, 133.8, 135.7 (2C), 135.8 (2C), 136.0, 170.3; HRMS (ESI-TOF) calcd for $\mathrm{C}_{45} \mathrm{H}_{60} \mathrm{O}_{12} \mathrm{SiNa}[\mathrm{M}+\mathrm{Na}]^{+}$843.3746, found 843.3754. 


\section{Computational modelling of B}

General method: For the global analysis of the conformational isomers of $\mathbf{B}$, the structures were created by the conformational search using molecular mechanics simulations (MacroModel ver.10.5) ${ }^{\mathrm{S} 15}$ and the DFT calculations (Gaussian 09 program). ${ }^{\mathrm{S} 16}$ The relative Gibbs energies ( $\Delta G, 298 \mathrm{~K}, 1 \mathrm{~atm}$ ), which were computed for gas phase, were given in $\mathrm{kcal} / \mathrm{mol}$. The computational studies were conducted using Fujitsu PRIMAERGY CX400 (Information Technology Center of Nagoya University).

\section{Optimization of B}

The conformational search of the corresponding C5-cation structure of $\mathbf{B}$ was first conducted by molecular mechanics simulation. The calculation was performed using a 1000-step of Monte Carlo based torsional sampling (MCMM) and Polak-Ribiere Conjugate Gradient (PRCG) energy minimization with OPLS2005 force field (gas phase) to afford 50 conformational isomers within $21 \mathrm{~kJ} / \mathrm{mol}$. All the cation structures were transferred into Gaussian 09 program and changed into the corresponding C5-radical structures. The preliminary geometry optimizations of the structures were performed at UPM6 level of theory (298 K, 1 atm, gas phase) to afford 22 conformational isomers. Then, the geometry optimizations of the thus-obtained structures were performed at UM06-2X/6-31+G(d) level of theory ( $298 \mathrm{~K}, 1 \mathrm{~atm}$, gas phase) to afford 21 conformational isomers. Among them, the lowest energy structure was defined as $\mathbf{B}$.

Energy, structure, and Cartesian coordinate of $\mathbf{B}$ :

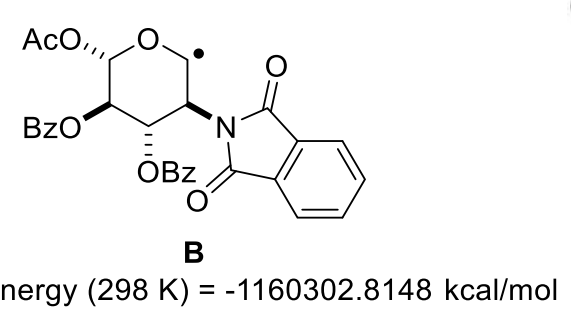

Gibbs free energy $(298 \mathrm{~K})=-1160302.8148 \mathrm{kcal} / \mathrm{mol}$

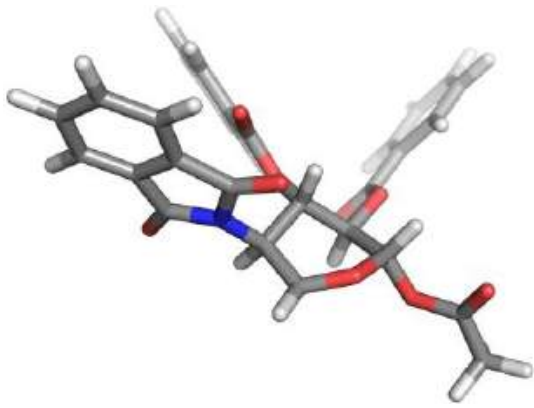

$-0.170570$

$-0.997087$

$-0.984572$

$-0.090047$

0.017262

$-0.488653$

$-0.765843$

0.196464 


\begin{tabular}{|c|c|c|c|}
\hline $\mathrm{O}$ & -0.061205 & 0.587565 & -0.546412 \\
\hline $\mathrm{N}$ & -2.454522 & -1.327689 & -0.540744 \\
\hline $\mathrm{C}$ & -2.901963 & -1.647199 & 0.749747 \\
\hline $\mathrm{C}$ & -4.239893 & -0.999736 & 0.887625 \\
\hline $\mathrm{C}$ & -4.492515 & -0.275154 & -0.271706 \\
\hline $\mathrm{C}$ & -3.322722 & -0.434584 & -1.185851 \\
\hline $\mathrm{O}$ & -2.288422 & -2.314819 & 1.547116 \\
\hline $\mathrm{O}$ & -3.118756 & 0.082409 & -2.258548 \\
\hline $\mathrm{C}$ & -5.140371 & -1.029675 & 1.937784 \\
\hline $\mathrm{C}$ & -6.322973 & -0.298520 & 1.784271 \\
\hline $\mathrm{C}$ & -6.576942 & 0.431160 & 0.618115 \\
\hline $\mathrm{C}$ & -5.656939 & 0.455107 & -0.435256 \\
\hline $\mathrm{C}$ & 2.904562 & 0.420059 & -0.490503 \\
\hline $\mathrm{C}$ & -0.870804 & 1.440417 & 0.117160 \\
\hline $\mathrm{O}$ & -1.676119 & 1.081027 & 0.946274 \\
\hline $\mathrm{C}$ & -0.641779 & 2.851303 & -0.292687 \\
\hline $\mathrm{C}$ & 3.810822 & 1.223248 & 0.375295 \\
\hline $\mathrm{O}$ & 2.724012 & 0.623894 & -1.666657 \\
\hline $\mathrm{C}$ & 3.984149 & 0.942389 & 1.733310 \\
\hline $\mathrm{C}$ & 4.840401 & 1.732851 & 2.495138 \\
\hline $\mathrm{C}$ & 5.517444 & 2.799769 & 1.905050 \\
\hline $\mathrm{C}$ & 5.341442 & 3.079993 & 0.549314 \\
\hline $\mathrm{C}$ & 4.488972 & 2.292265 & -0.216588 \\
\hline $\mathrm{C}$ & 0.350200 & 3.195551 & -1.216223 \\
\hline $\mathrm{C}$ & 0.530295 & 4.533718 & -1.556564 \\
\hline $\mathrm{C}$ & -0.274046 & 5.517902 & -0.982516 \\
\hline $\mathrm{C}$ & -1.263864 & 5.169271 & -0.062223 \\
\hline $\mathrm{C}$ & -1.448446 & 3.835304 & 0.284555 \\
\hline $\mathrm{C}$ & 3.075068 & -4.440512 & -0.189451 \\
\hline $\mathrm{O}$ & 2.891551 & -4.790777 & 0.945520 \\
\hline $\mathrm{C}$ & 4.038623 & -5.054960 & -1.164413 \\
\hline $\mathrm{H}$ & -0.291739 & -0.872633 & 0.899755 \\
\hline $\mathrm{H}$ & -1.068091 & -1.184573 & -2.023486 \\
\hline $\mathrm{H}$ & -1.478617 & -3.782856 & -1.192302 \\
\hline $\mathrm{H}$ & 1.700846 & -2.799166 & 1.070129 \\
\hline $\mathrm{H}$ & 1.493554 & -1.291589 & -1.566957 \\
\hline $\mathrm{H}$ & -4.928216 & -1.594475 & 2.840189 \\
\hline $\mathrm{H}$ & -7.057036 & -0.293120 & 2.584338 \\
\hline $\mathrm{H}$ & -7.503945 & 0.990048 & 0.532540 \\
\hline $\mathrm{H}$ & -5.839069 & 1.021773 & -1.343139 \\
\hline $\mathrm{H}$ & 3.451886 & 0.112319 & 2.184787 \\
\hline $\mathrm{H}$ & 4.977785 & 1.517099 & 3.550189 \\
\hline $\mathrm{H}$ & 6.183159 & 3.415136 & 2.503300 \\
\hline $\mathrm{H}$ & 5.868349 & 3.911649 & 0.091707 \\
\hline $\mathrm{H}$ & 4.335464 & 2.489381 & -1.273177 \\
\hline $\mathrm{H}$ & 0.970720 & 2.422395 & -1.659753 \\
\hline $\mathrm{H}$ & 1.298745 & 4.807687 & -2.273067 \\
\hline $\mathrm{H}$ & -0.130049 & 6.560089 & -1.252985 \\
\hline $\mathrm{H}$ & -1.889197 & 5.937080 & 0.382983 \\
\hline $\mathrm{H}$ & -2.209436 & 3.537307 & 0.999385 \\
\hline $\mathrm{H}$ & 3.482020 & -5.470659 & -2.009010 \\
\hline $\mathrm{H}$ & 4.712172 & -4.288348 & -1.555834 \\
\hline $\mathrm{H}$ & 4.604160 & -5.840957 & -0.66588 \\
\hline
\end{tabular}




\section{CP3 analysis of 6- $\alpha$ and 6- $\beta$}

The ${ }^{1} \mathrm{H}$ NMR chemical shifts of compounds $\mathbf{6}-\boldsymbol{\alpha}$ and $\mathbf{6}-\boldsymbol{\beta}$ were calculated according to the protocol reported by Hehre and Hashimoto et al. ${ }^{\text {S17 }}$

For the global analyses of the conformational isomers of $\mathbf{6 - \alpha}$ and $\mathbf{6 - \beta} \boldsymbol{\beta}$, the structures were created by the conformational search using molecular mechanics simulations (MacroModel ver.10.5). Each calculation was performed using a 1000-step of MCMM and PRCG energy minimization with MMFF $\left(\mathrm{CHCl}_{3}\right.$ solvent phase) to afford $252(\mathbf{6}-\boldsymbol{\alpha})$ and $319(\mathbf{6}-\boldsymbol{\beta})$ conformational isomers within $21 \mathrm{~kJ} / \mathrm{mol}$. In addition, the candidate structures were narrowed down to $37(\mathbf{6}-\boldsymbol{\alpha})$ and $26(\mathbf{6}-\boldsymbol{\beta})$ conformational isomers by excluding those that did not converge or were observed less than 10 times. The thus-obtained structures of $\mathbf{6}-\boldsymbol{\alpha}$ and $\mathbf{6}-\boldsymbol{\beta}$ were transferred into Gaussian 09 program. The following calculation steps were performed at the each specified level of theory $(298 \mathrm{~K}, 1 \mathrm{~atm})$ with solvation effects using the IEFPCM solvation model $\left(\mathrm{CHCl}_{3}\right)$.

1. Structure optimization employing the HF/3-21G model, followed by conformer narrowing by setting the threshold at $40 \mathrm{~kJ} / \mathrm{mol}$ from the global minimum conformational isomer (37 (6$\boldsymbol{\alpha})$ and 26 (6- $\boldsymbol{\beta})$ conformational isomers).

2. Energy estimation with fixing the geometries using the $\omega \mathrm{B} 97 \mathrm{X}-\mathrm{D} / 6-31 \mathrm{G}(\mathrm{d})$ model, followed by conformer narrowing by setting the threshold at $15 \mathrm{~kJ} / \mathrm{mol}$ from the global minimum conformational isomer (23 (6- $\boldsymbol{\alpha})$ and 15 (6- $\boldsymbol{\beta})$ conformational isomers).

3. Structural optimization with the $\omega \mathrm{B} 97 \mathrm{X}-\mathrm{D} / 6-31 \mathrm{G}(\mathrm{d})$ model, followed by conformer narrowing by setting the threshold at $10 \mathrm{~kJ} / \mathrm{mol}$ from the global minimum conformational isomer (10 (6- $\boldsymbol{\alpha})$ and 5 (6- $\boldsymbol{\beta})$ conformational isomers).

4. Energy estimation employing the $\omega$ B97X-D/6-311+G(2df,2p) model, followed by conformer narrowing by setting the threshold at $10 \mathrm{~kJ} / \mathrm{mol}$ from the global minimum conformational isomer (10 (6- $\boldsymbol{\alpha})$ and 4 (6- $\boldsymbol{\beta})$ conformational isomers).

5. Chemical shift calculations using $\omega \mathrm{B} 97 \mathrm{X}-\mathrm{D} / 6-31 \mathrm{G}(\mathrm{d})$ without empirical correction.

The calculated chemical shifts of $\mathbf{6}-\boldsymbol{\alpha}$ and $\mathbf{6}-\boldsymbol{\beta}$ were corrected by considering the Boltzmann weighting and the calculated chemical shift of tetramethylsilane at the $\omega B 97 X-D / 6-31 G(d)$ level of theory. The experimental and calculated chemical shifts were summarized in Table $\mathrm{S} 5$. The CP3 analysis was summarized in Table S6. ${ }^{\mathrm{S} 18}$ 
Table S5. Experimental and calculated ${ }^{1} \mathrm{H}$ NMR chemical shifts of $\mathrm{H} 1-\mathrm{H} 11$ and $\mathrm{CH}_{3} \mathrm{CO}$ of 6$\alpha$ and 6- $\beta$

\begin{tabular}{|c|c|c|c|c|}
\hline \multirow[b]{2}{*}{ position } & \multicolumn{2}{|c|}{$\delta_{\exp }\left({ }^{1} \mathrm{H}\right), \mathrm{ppm}^{a}\left[\mathrm{CDCl}_{3}, 400 \mathrm{MHz}\right]$} & \multicolumn{2}{|c|}{$\delta_{\text {calcd }}\left({ }^{1} \mathrm{H}\right), \mathrm{ppm}^{b}$} \\
\hline & $6-\alpha$ & $6-\beta$ & $6-\alpha$ & $6-\beta$ \\
\hline $\mathrm{H} 1$ & 6.13 & 6.17 & 5.61 & 5.83 \\
\hline $\mathrm{H} 2$ & 5.60 & 5.65 & 5.23 & 4.17 \\
\hline $\mathrm{H} 3$ & 6.38 & 6.30 & 5.70 & 7.00 \\
\hline $\mathrm{H} 4$ & 4.71 & 5.03 & 4.20 & 4.31 \\
\hline $\mathrm{H} 5$ & 4.64 & 4.90 & 4.81 & 5.05 \\
\hline H6 & 3.74 & 3.54 & 3.55 & 3.31 \\
\hline $\mathrm{H} 7$ & 4.05 & 3.97 & 4.04 & 3.90 \\
\hline $\mathrm{H} 8$ & 4.05 & 3.97 & 3.84 & 3.81 \\
\hline H9 & 3.78 & 3.88 & 3.80 & 3.54 \\
\hline $\mathrm{H} 10$ & 4.05 & 4.06 & 3.76 & 3.63 \\
\hline $\mathrm{H} 11 \mathrm{a}$ & 4.34 & 4.37 & 3.55 & 5.05 \\
\hline $\mathrm{H} 11 \mathrm{~b}$ & 4.44 & 4.45 & 3.65 & 3.90 \\
\hline $\mathrm{CH}_{3} \mathrm{CO}$ & 2.06 & 2.06 & 2.20 & 0.88 \\
\hline
\end{tabular}

${ }^{a}$ Chemical shifts were reported in ppm on the $\delta$ scale relative to $\mathrm{CHCl}_{3}(\delta=7.26)$.

${ }^{b} \mathrm{Chemical}$ shift data were produced using tetramethylsilane (TMS) as reference compound for $\mathrm{C}\left(\mathrm{sp}^{3}\right)$-hydrogens.

Table S6. CP3 analysis of $\mathbf{6}-\boldsymbol{\alpha}$ and $\mathbf{6}-\boldsymbol{\beta}$ based on ${ }^{1} \mathrm{H}$ NMR data.

\begin{tabular}{c|c} 
& probability \\
\hline $\mathbf{6}-\boldsymbol{\alpha}(\exp )-\mathbf{6}-\boldsymbol{\alpha}($ calcd $)$ and $\mathbf{6}-\boldsymbol{\beta}(\exp )-\mathbf{6}-\boldsymbol{\beta}($ calcd $)$ & $90.3 \%$ \\
$\mathbf{6}-\boldsymbol{\alpha}(\exp )-\mathbf{6}-\boldsymbol{\beta}($ calcd $)$ and $\mathbf{6}-\boldsymbol{\beta}(\exp )-\mathbf{6}-\boldsymbol{\alpha}($ calcd $)$ & $9.7 \%$
\end{tabular}




\section{References}

S1. Zinner, H.; Wessely, K.; Bock, W.; Rieckhoff, K.; Strandt, F.; Nimmich, W. Chem. Ber. 1957, 90, 500 .

S2. $\quad$ Delbrouck, J. A.; Bochatay, V. N.; Tikad, A.; Vincent, S. P. Org. Lett. 2019, 21, 5562.

S3. Dondoni, A.; Marra, A.; Merino, P. J. Am. Chem. Soc. 1994, 116, 3324.

S4. Yamamoto, K.; Sato, Y.; Ishimori, A.; Miyairi, K.; Okuno, T.; Nemoto, N.; Shimizu, H.; Kidokoro, S.; Hashimoto, M. Biosci. Biotechnol. Biochem. 2008, 72, 2039.

S5. Nagatomo, M.; Kamimura, D.; Matsui, Y.; Masuda, K.; Inoue, M. Chem. Sci. 2015, 6, 2765 .

S6. Compounds $\mathbf{S} \mathbf{4}$ and $\mathbf{1}$ have a propensity to form the corresponding borates with $\mathrm{B}_{2} \mathrm{O}_{3}$ leached from borosilicate glassware (Pyrex glass). Therefore, these reactions were carried out in quartz glass flask, and soda-lime glassware was used for the purification, and quartz glass tube was used for the NMR experiments. For a related example, see: Nagatomo, M.; Koshimizu, M.; Masuda, K.; Tabuchi, T.; Urabe, D.; Inoue, M. J. Am. Chem. Soc. 2014, 136, 5916.

S7. (a) Schroeder, A. C.; Hughes, R. G. Jr.; Bloch, A. J. Med. Chem. 1981, 24, 1078. (b) Okabe, M.; Sun, R.-C.; Tam, S. Y.-K.; Todaro, L. J.; Coffen, D. L. J. Org. Chem. 1988, 53,4780 .

S8. Leon, B.; Liemann, S.; Klaffke, W. J. Carbohydr. Chem. 1993, 12, 597.

S9. Hainrichson, M.; Pokrovskaya, V.; Shallom-Shezifi, D.; Fridman, M.; Belakhov, V.; Shachar, D.; Yaron, S.; Baasov, T. Bioorg. Medchem. Lett. 2005, 13, 5797.

S10 Faghih, R.; Escribano, F. C.; Castillon, S.; Garcia, J.; Lukacs, G.; Olesker, A.; Thang, T. T. J. Org. Chem. 1986, 51, 4558.

S11. Wang, A-P.; Liu, C.; Yang, S.; Zhao, Z.; Lei, P. Tetrahedron 2016, 72, 285.

S12. (a) Ikemoto, N.; Schreiber, S. L. J. Am. Chem. Soc. 1990, 112, 9657. (b) Ikemoto, N.; Schreiber, S. L. J. Am. Chem. Soc. 1992, 114, 2524.

S13 Matsumori, N.; Kaneno, D.; Murata, M.; Nakamura, H.; Tachibana, K. J. Org. Chem. 1999, 64, 866 .

S14 Trost, B. M.; Belletire, J. L.; Godleski, S.; McDougal, P. G.; Balkovec, J. M. J. Org. Chem. 1986, 51, 2370.

S15. (a) MacroModel, version 10.5, Schrödinger, LLC, New York, NY, 2014. (b) Mohamadi, F.; Richards, N. G. J.; Guida, W. C.; Liskamp, R.; Lipton, M.; Caufield, C.; Chang, G.; 
Hendrickson, T.; Still, W. C. J. Comput. Chem. 1990, 11, 440.

S16. Frisch, M. J.; Trucks. G. W.; Schlegel, H. B.; Scuseria G. E.; Robb, M. A.; Cheeseman, J. R.; Scalmani, G.; Barone, V.; Mennucci, B.; Petersson, G. A.; Nakatsuji, H.; Caricato, M.; Li, X.; Hratchian, H. P.; Izmaylov, A. F.; Bloino, J.; Zheng, G.; Sonnenberg, J. L.; Hada, M.; Ehara, M.; Toyota, K.; Fukuda, R.; Hasegawa, J.; Ishida, M.; Nakajima, T.; Honda, Y.; Kitao, O.; Nakai, H.; Vreven, T.; Montgomery, Jr., J. A.; Peralta, J. E.; Ogliaro, F.; Bearpark, M.; Heyd, J. J.; Brothers, E.; Kudin, K. N.; Staroverov, V. N.; Keith, T.; Kobayashi, R.; Normand, J.; Raghavachari, K.; Rendell, A.; Burant, J. C.; Iyengar, S. S.; Tomasi, J.; Cossi, M.; Rega, N.; Millam, J. M.; Klene, M.; Knox, J. E.; Cross, J. B.; Bakken, V.; Adamo, C.; Jaramillo, J.; Gomperts, R.; Stratmann. R. E.; Yazyev, O.; Austin, A. J.; Cammi, R.; Pomelli, C.; Ochterski, J. W.; Martin, R. L.; Morokuma, K.; Zakrzewski, V. G.; Voth G. A.; Salvador, P.; Dannenberg, J. J.; Dapprich, S.; Daniels, A. D.; Farkas, O.; Foresman, J. B.; Ortiz, J. V.; Cioslowski, J.; Fox, D. J. Gaussian 09 (Revision E.01), Gaussian Inc., Wallingford, CT, 2013.

S17. Hehre, W.; Klunzinger, P.; Deppmeier, B.; Driessen, A.; Uchida, N.; Hashimoto, M.; Fukushi, E.; Takata, Y. J. Nat. Prod. 2019, 82, 2299.

S18. The analysis was done using an applet on a website published by Goodman. http://wwwjmg.ch.cam.ac.uk/tools/nmr/CP3.html 

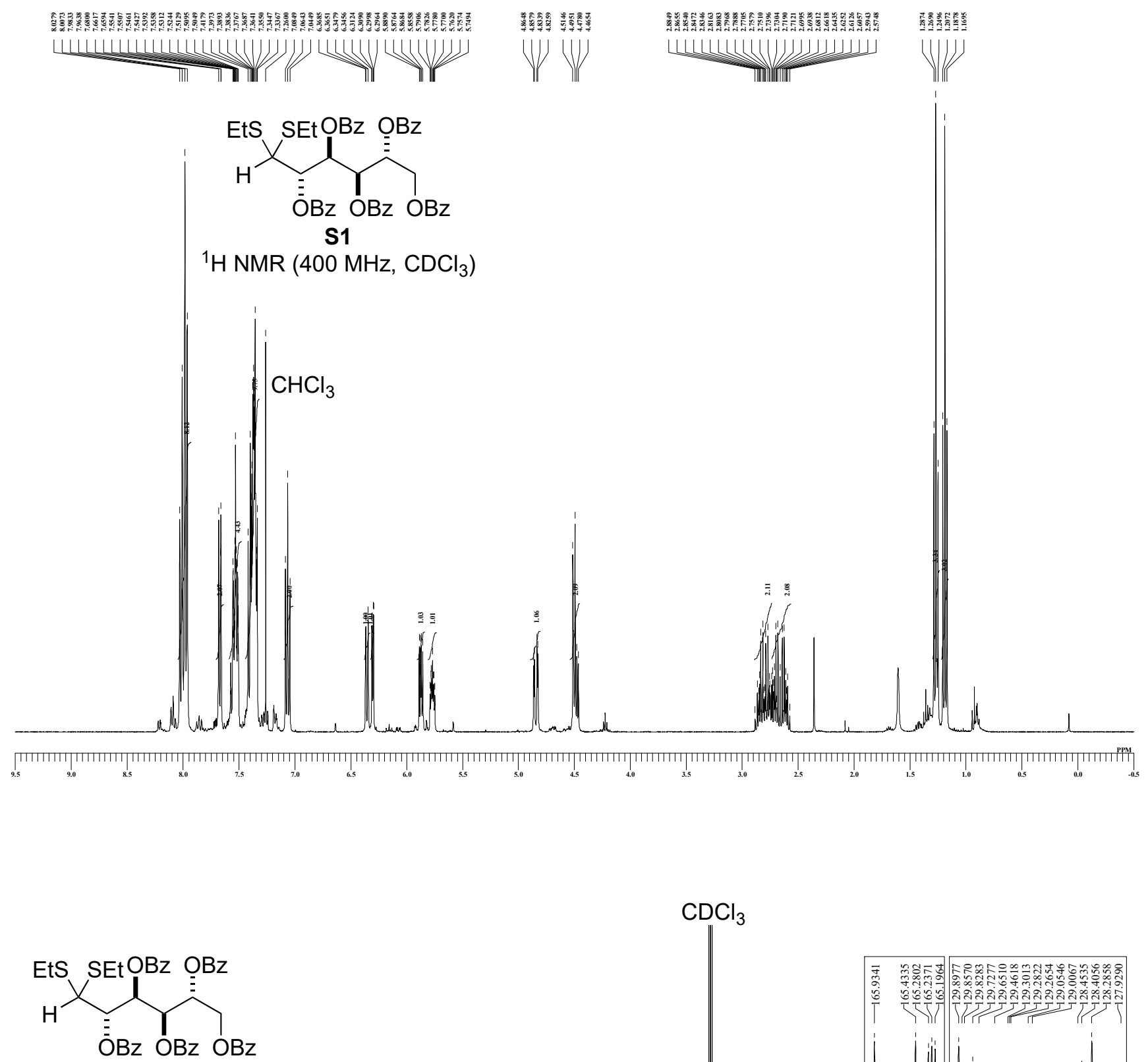

S1

${ }^{13} \mathrm{C}$ NMR $\left(100 \mathrm{MHz}, \mathrm{CDCl}_{3}\right)$
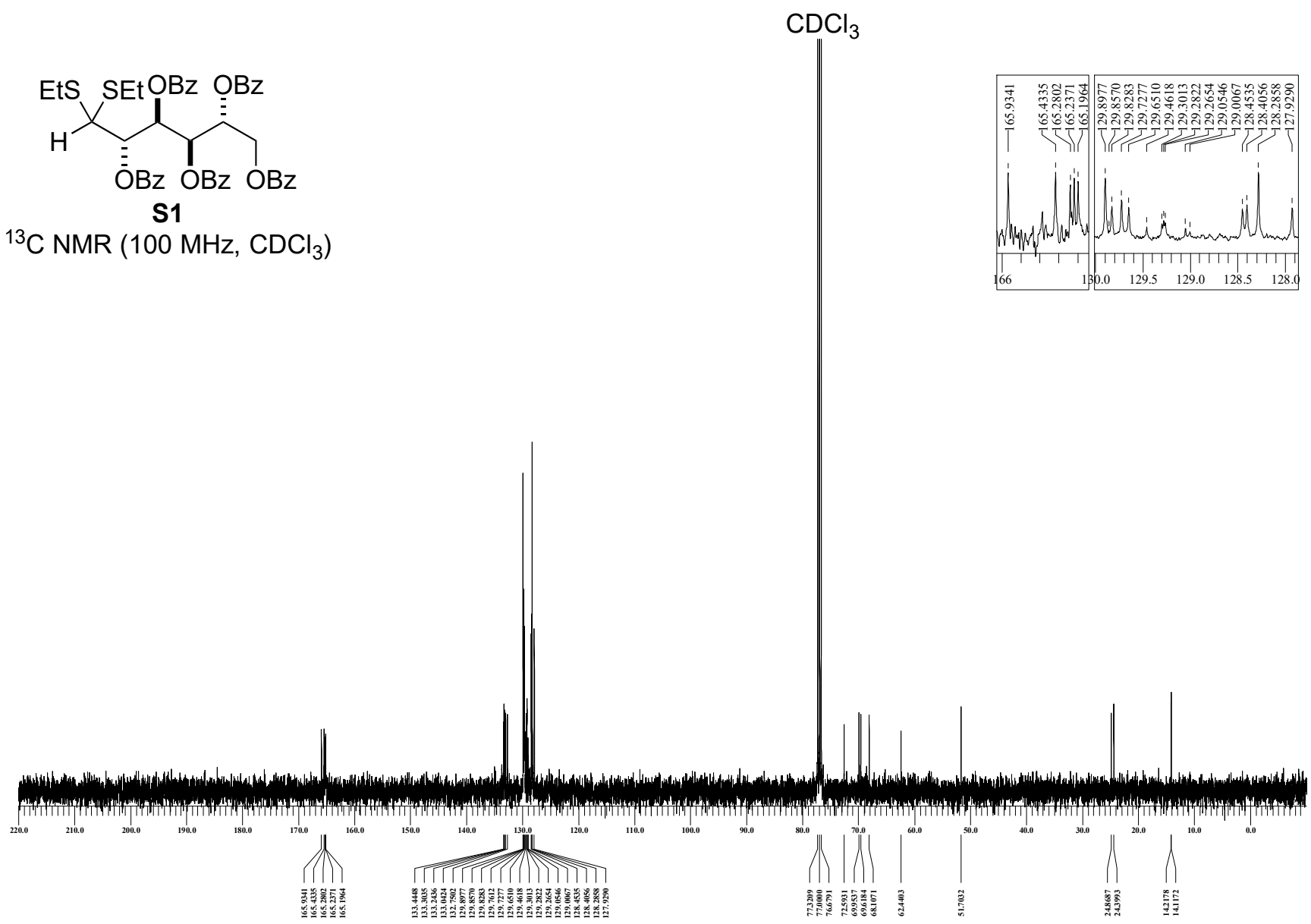

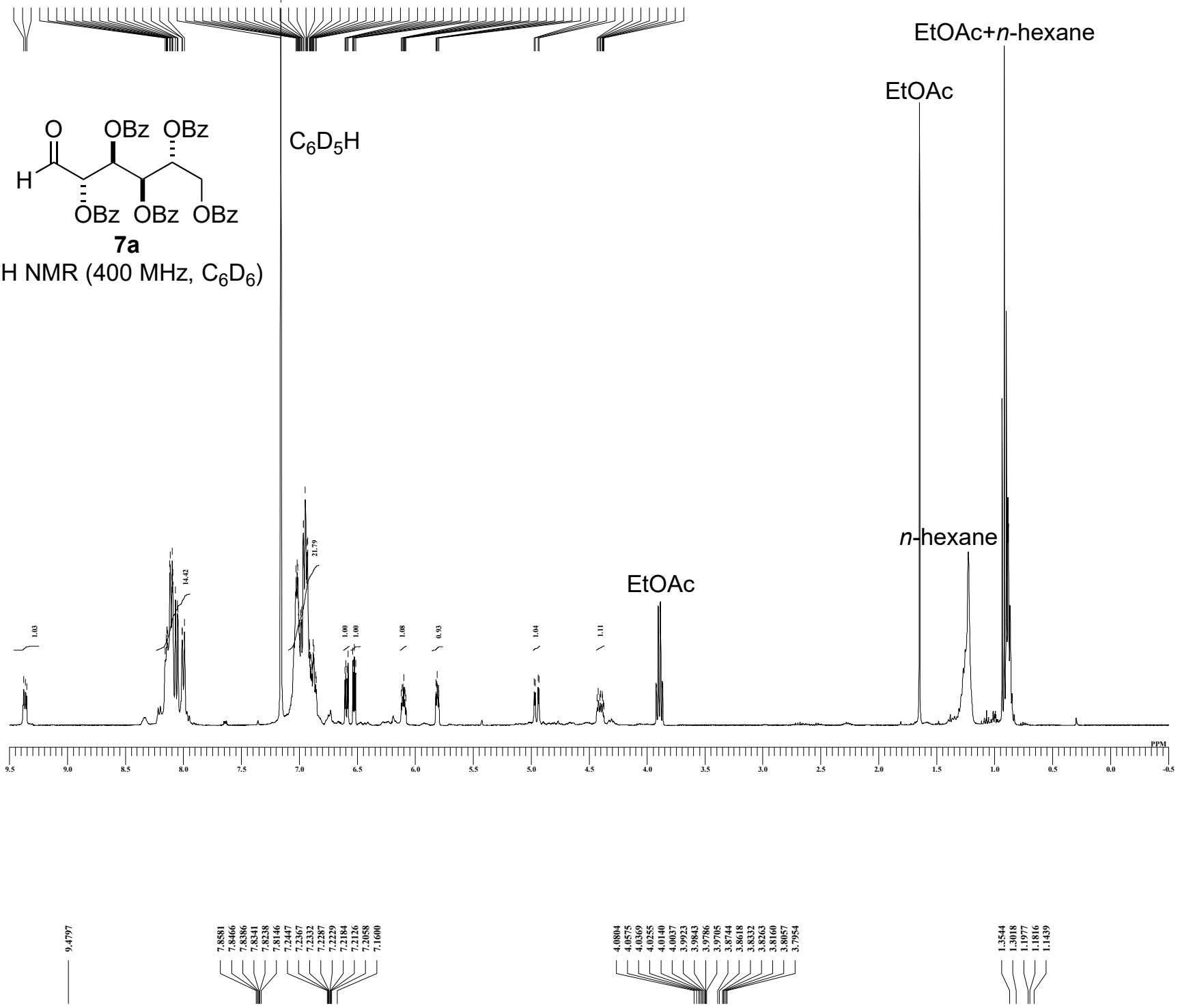<smiles>CC1(C)OC(CO[PbH2])C2OC(C)(C)OC(C=O)C2O1</smiles>

${ }^{1} \mathrm{H}$ NMR $\left(400 \mathrm{MHz}, \mathrm{C}_{6} \mathrm{D}_{6}\right)$
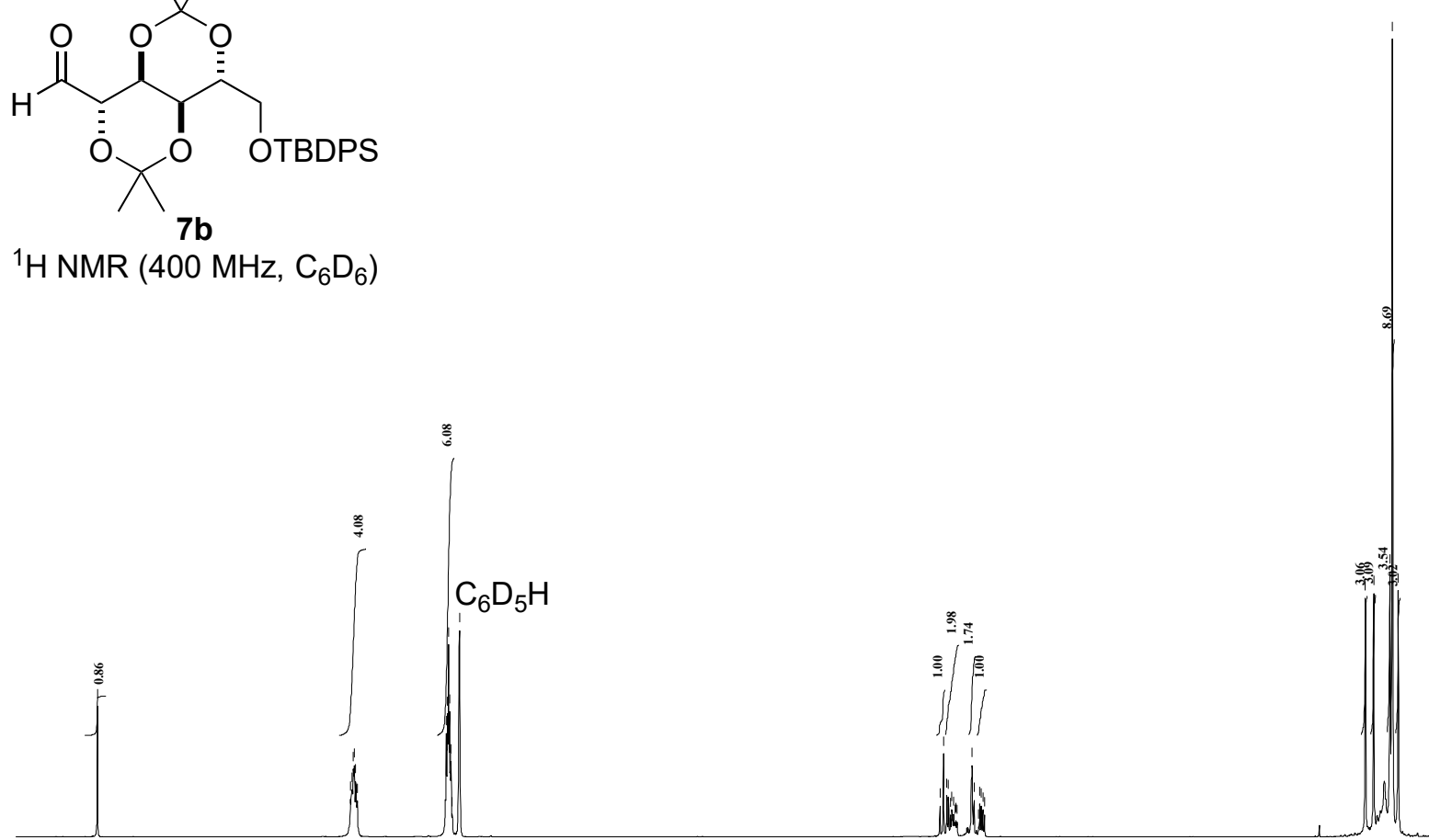

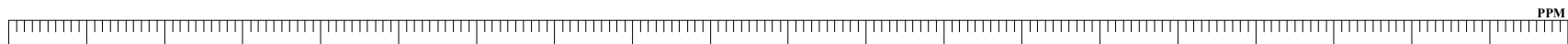



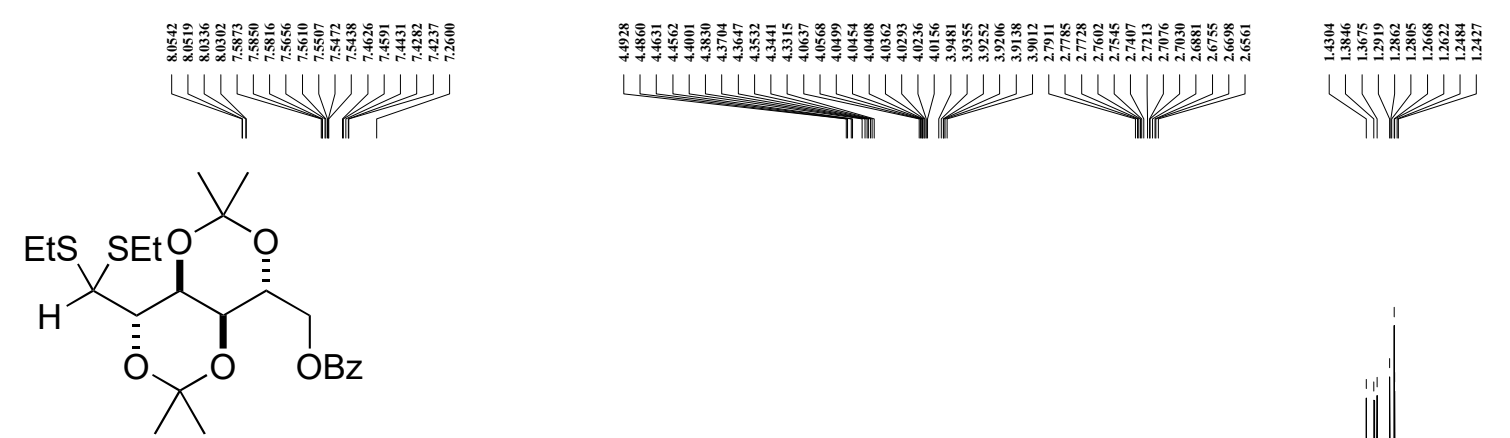

26

${ }^{1} \mathrm{H}$ NMR (400 MHz, $\mathrm{CDCl}_{3}$ )
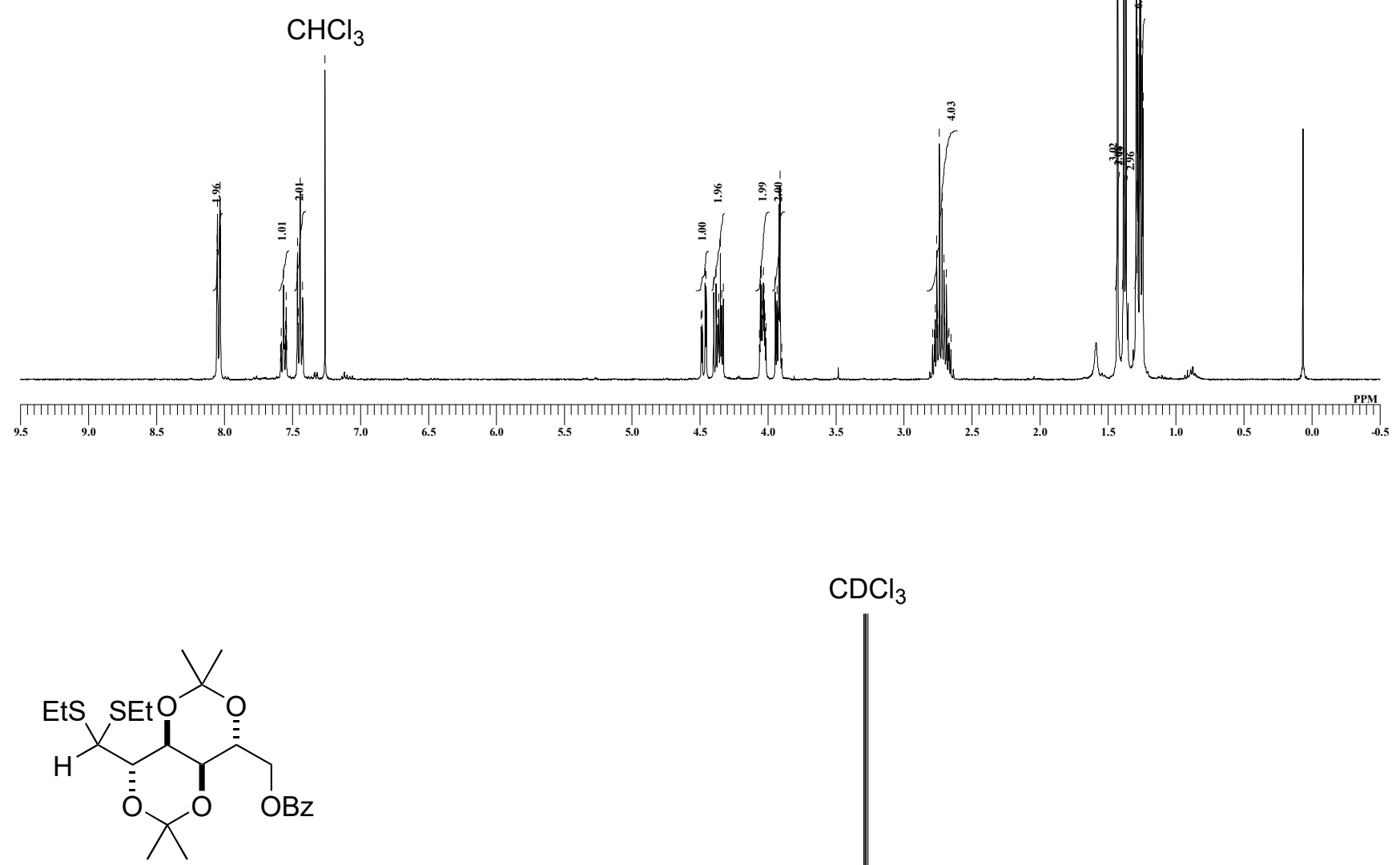

26

${ }^{13} \mathrm{C}$ NMR $\left(100 \mathrm{MHz}, \mathrm{CDCl}_{3}\right)$

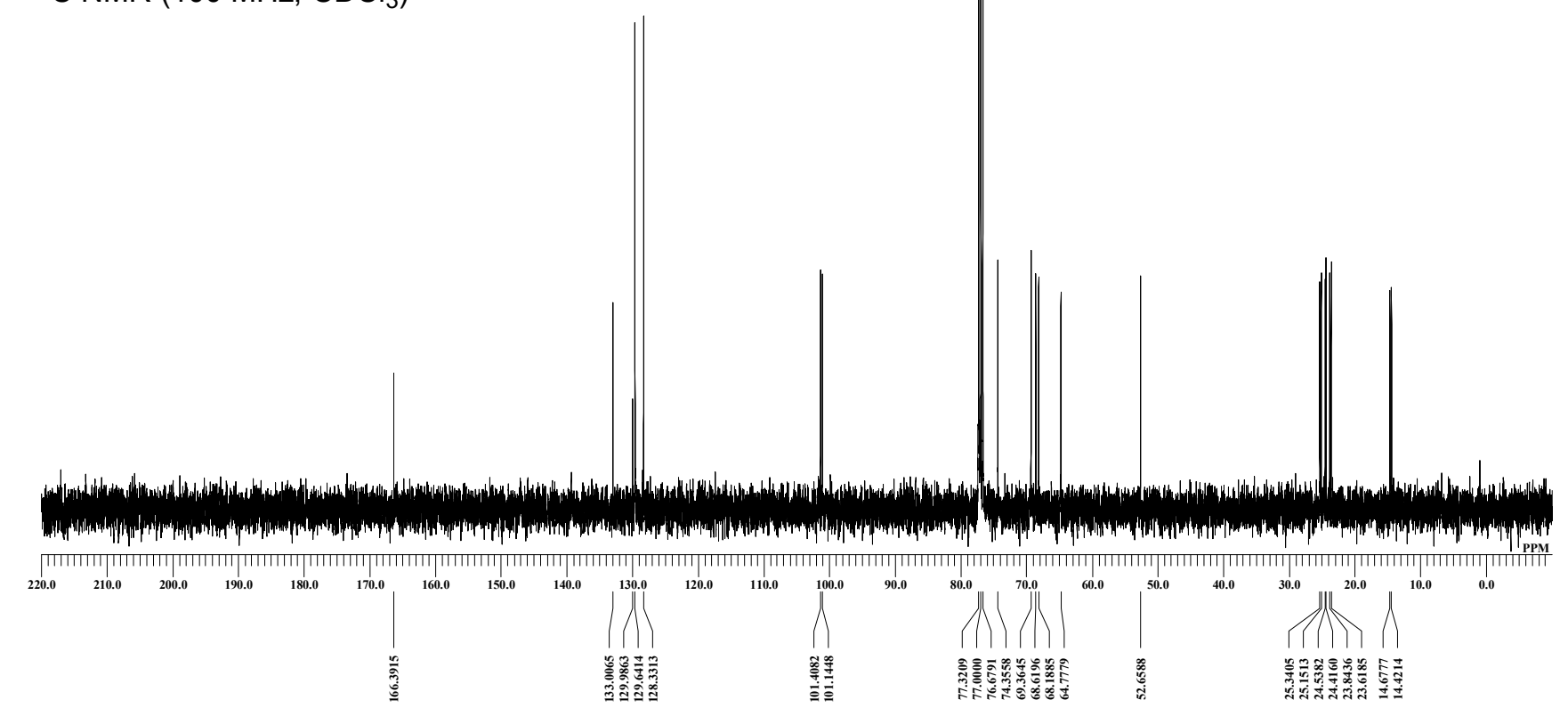


<smiles>CC(C)(C)OC[C@H]1OC(C)(C)O[C@@H]2C(C=O)OC(C)(C)O[C@@H]21</smiles>

$$
7 \mathrm{c}
$$

${ }^{1} \mathrm{H}$ NMR $\left(400 \mathrm{MHz}, \mathrm{C}_{6} \mathrm{D}_{6}\right)$
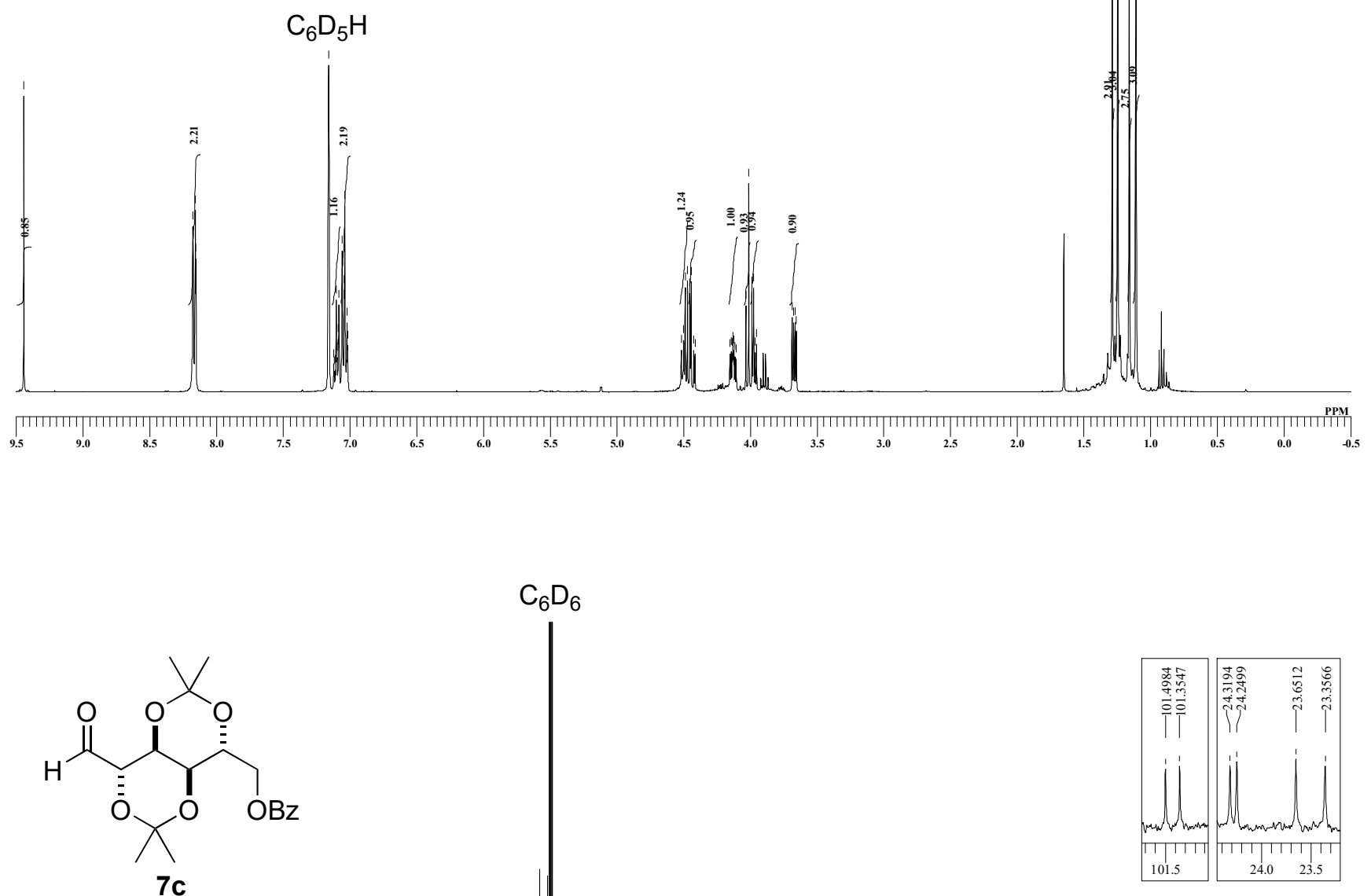

\section{$\mathrm{C}_{6} \mathrm{D}_{6}$}

${ }^{13} \mathrm{C}$ NMR (100 MHz, $\left.\mathrm{C}_{6} \mathrm{D}_{6}\right)$
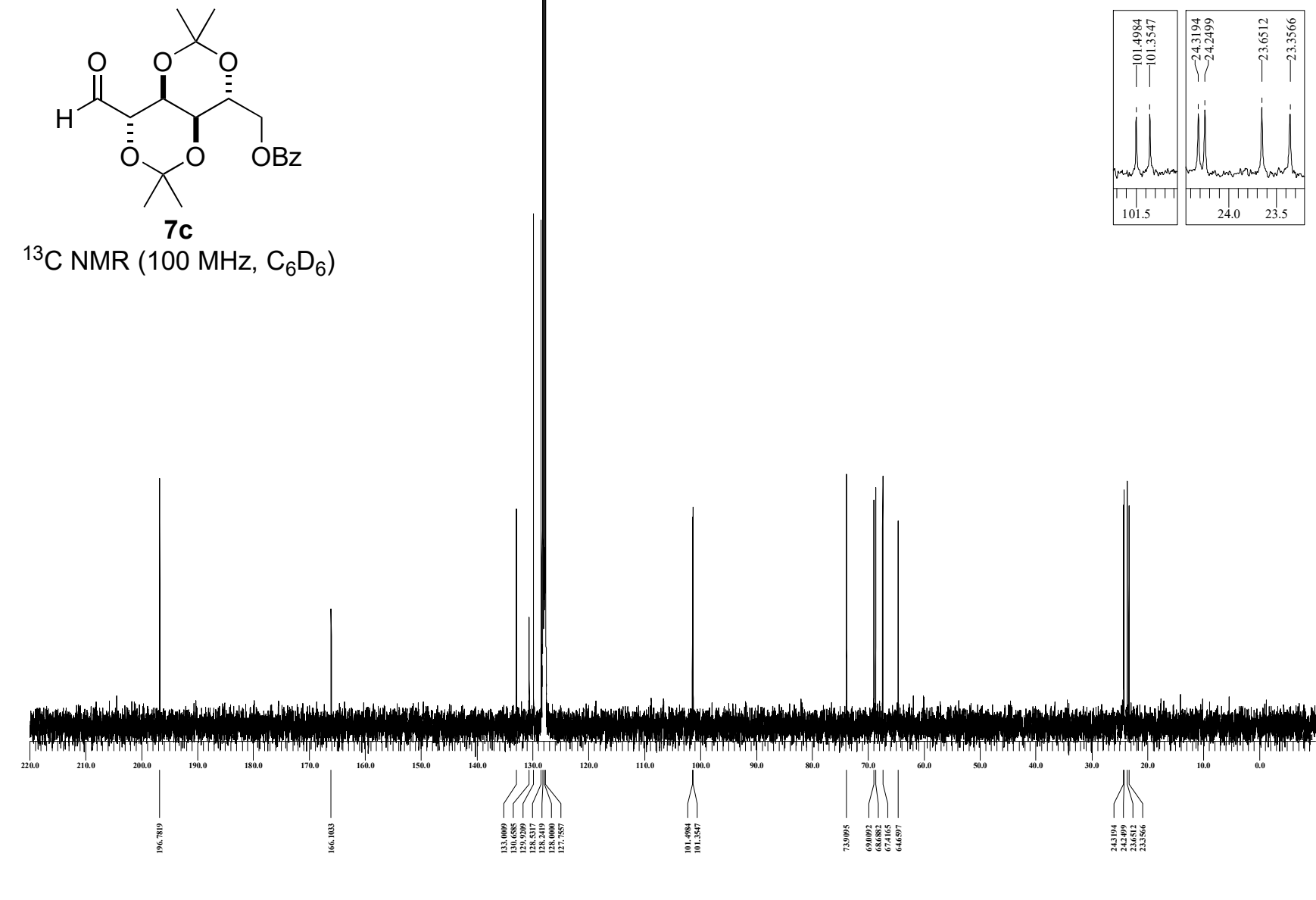

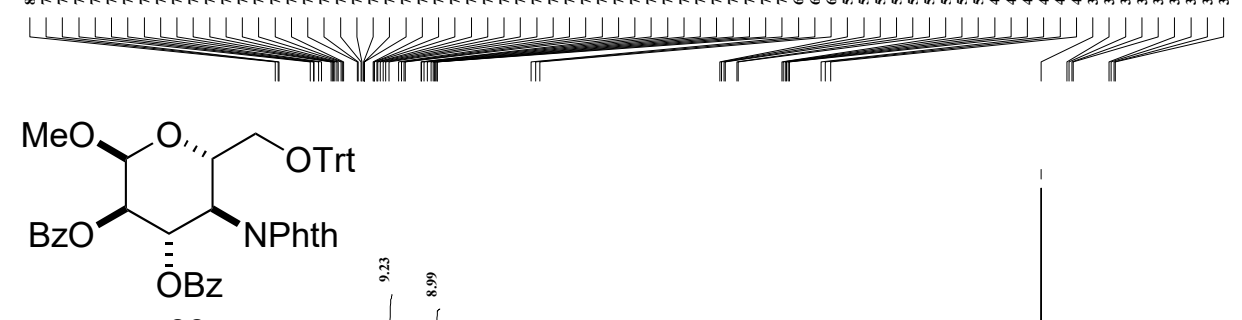

33

${ }^{1} \mathrm{H}$ NMR

$\left(400 \mathrm{MHz}, \mathrm{CDCl}_{3}\right)$

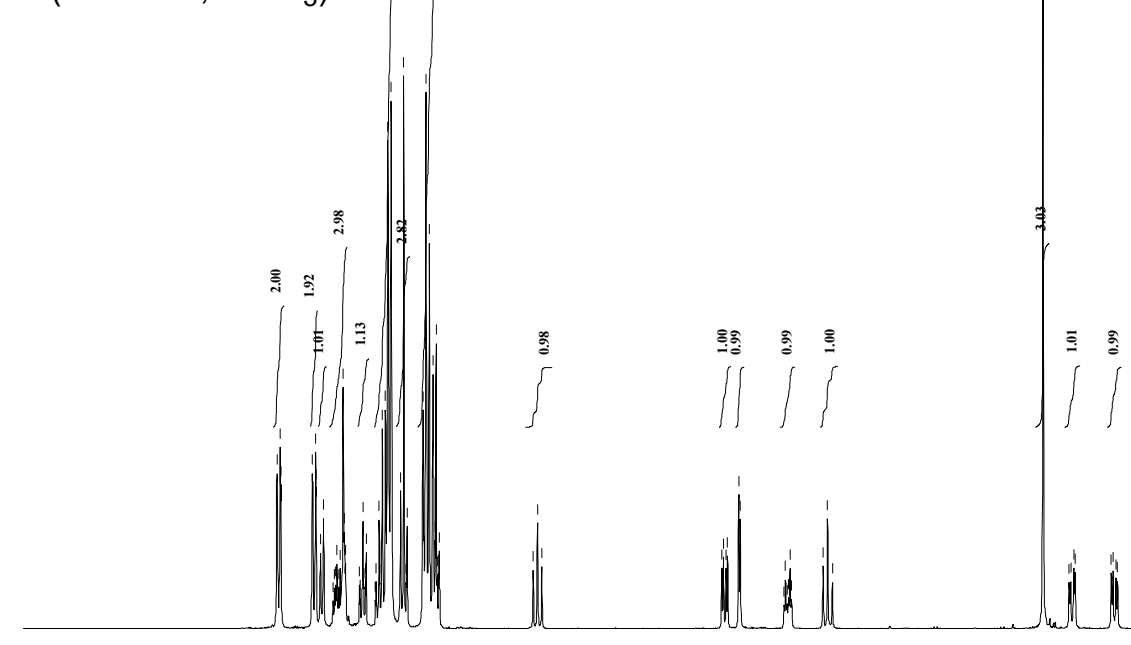

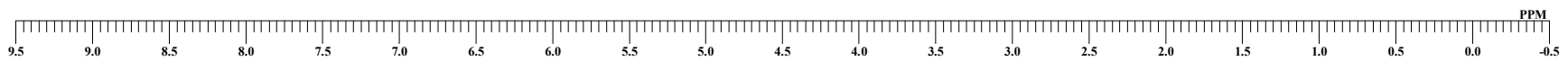<smiles>CCCOC(=O)c1ccccc1</smiles>

33

${ }^{13} \mathrm{C}$ NMR $\left(100 \mathrm{MHz}, \mathrm{CDCl}_{3}\right)$
$\mathrm{CDCl}_{3}$

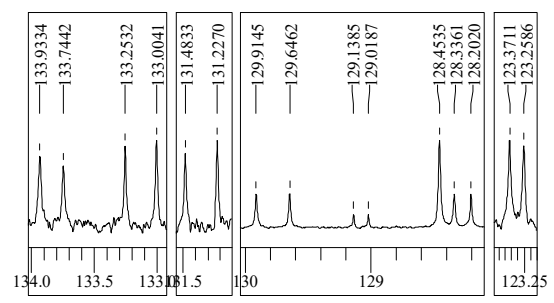

W W

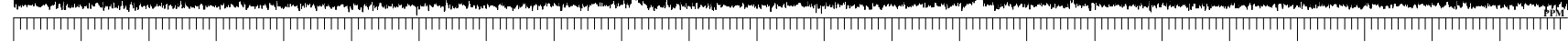




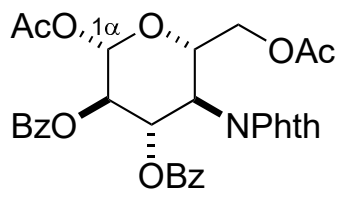

34- $\alpha$

34- $\alpha: 34-\beta=1: 8.3$

${ }^{1} \mathrm{H}$ NMR $\left(400 \mathrm{MHz}, \mathrm{CDCl}_{3}\right)$

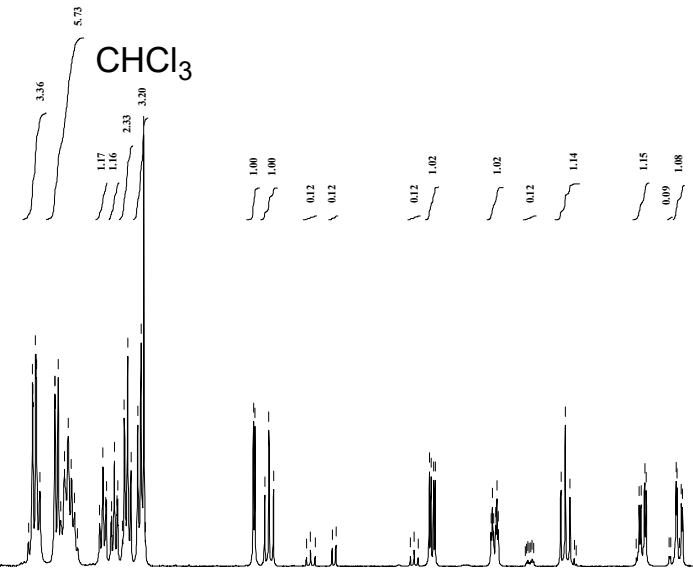

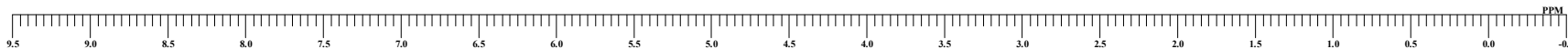

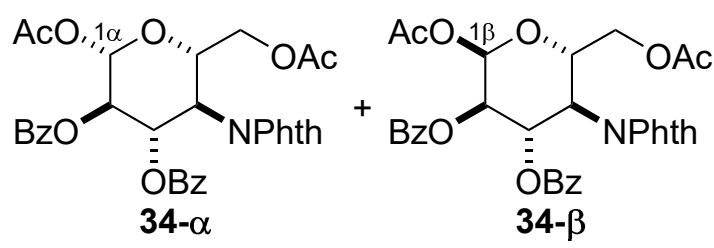

34- $\alpha: 34-\beta=1: 8.3$

${ }^{13} \mathrm{C} \mathrm{NMR}\left(100 \mathrm{MHz}, \mathrm{CDCl}_{3}\right)$
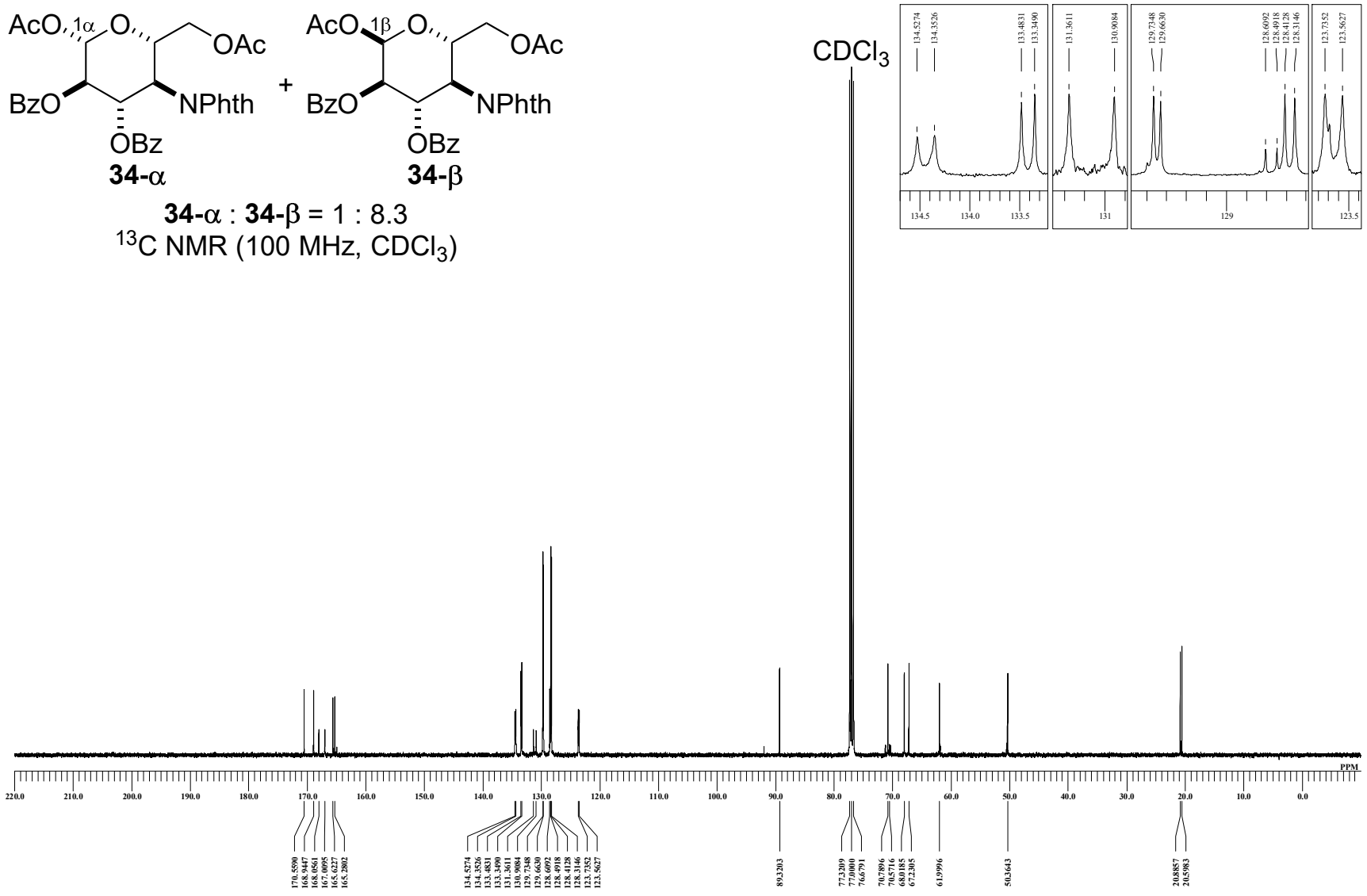


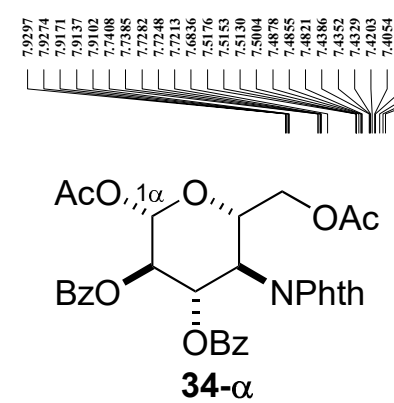

${ }^{1} \mathrm{H}$ NMR $\left(400 \mathrm{MHz}, \mathrm{CDCl}_{3}\right)$

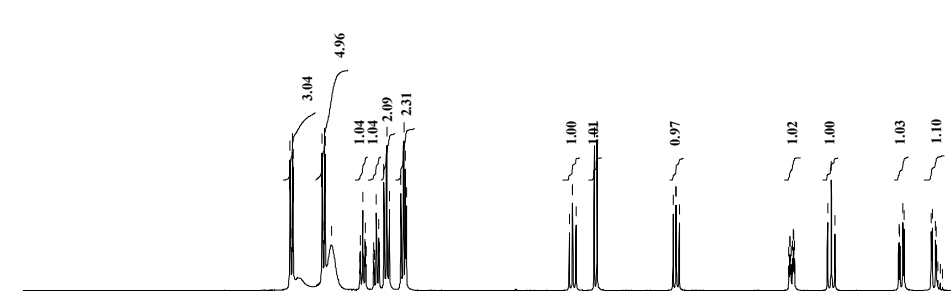

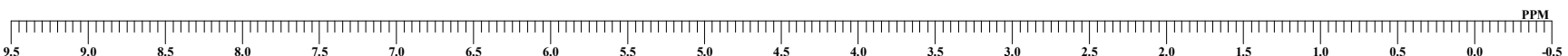

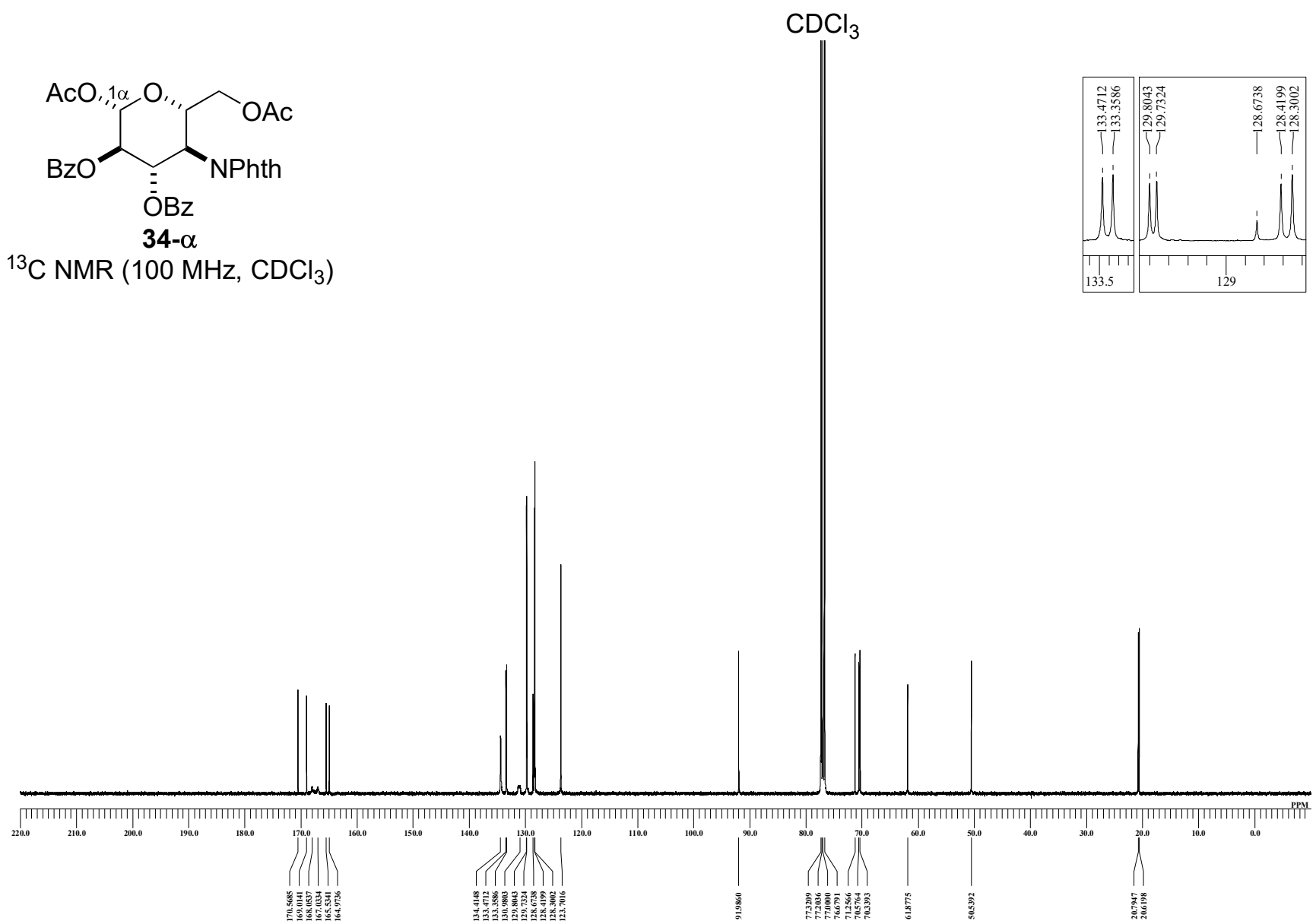



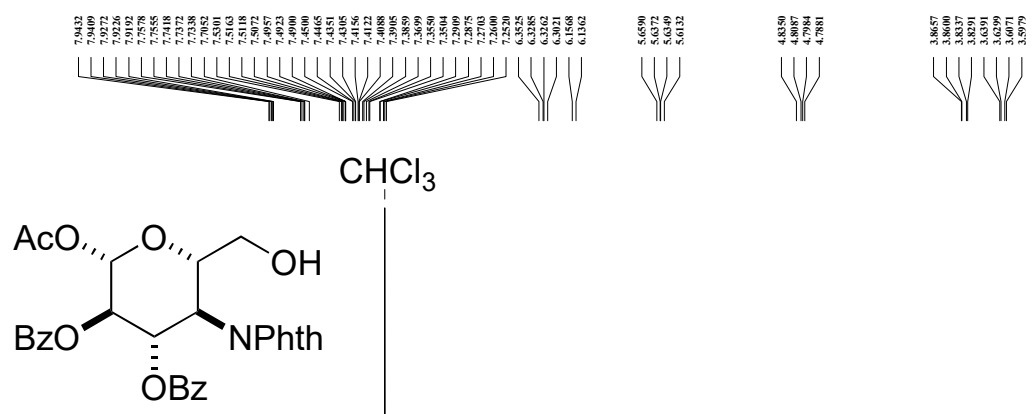

36

${ }^{1} \mathrm{H}$ NMR $\left(400 \mathrm{MHz}, \mathrm{CDCl}_{3}\right)$

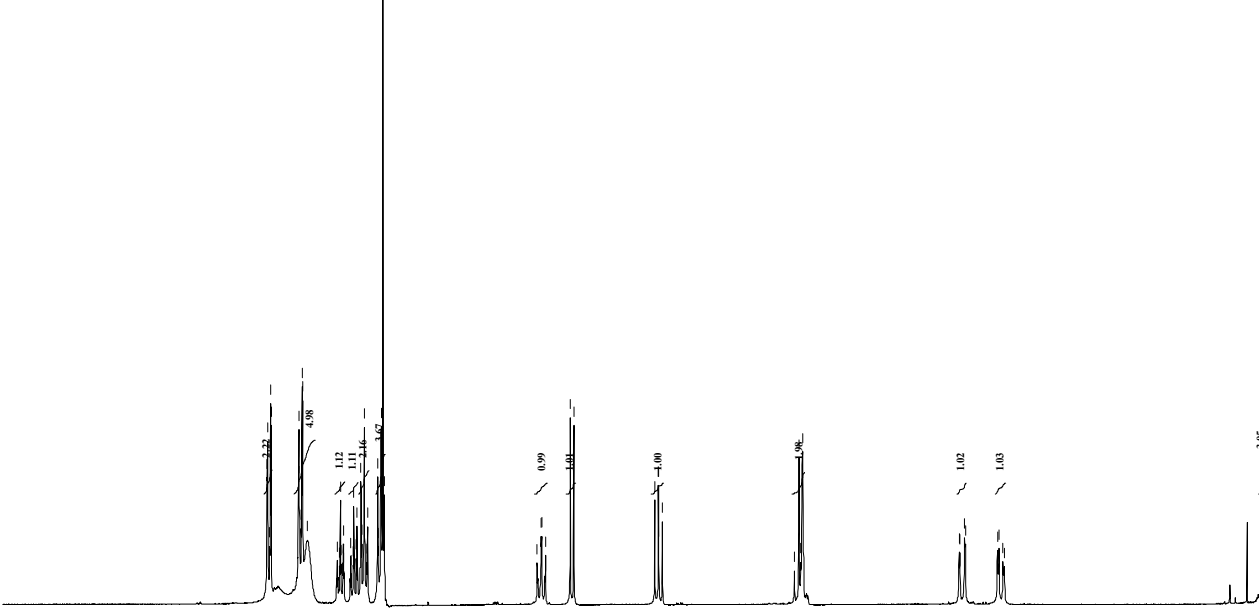

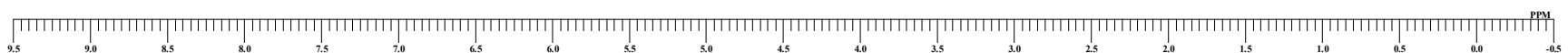

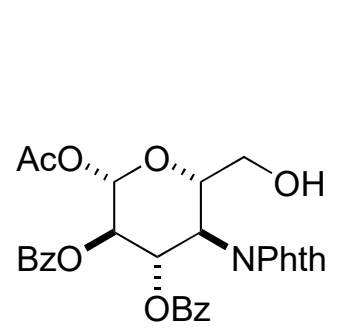

36

$\mathrm{CDCl}_{3}$

${ }^{13} \mathrm{C}$ NMR $\left(100 \mathrm{MHz}, \mathrm{CDCl}_{3}\right)$
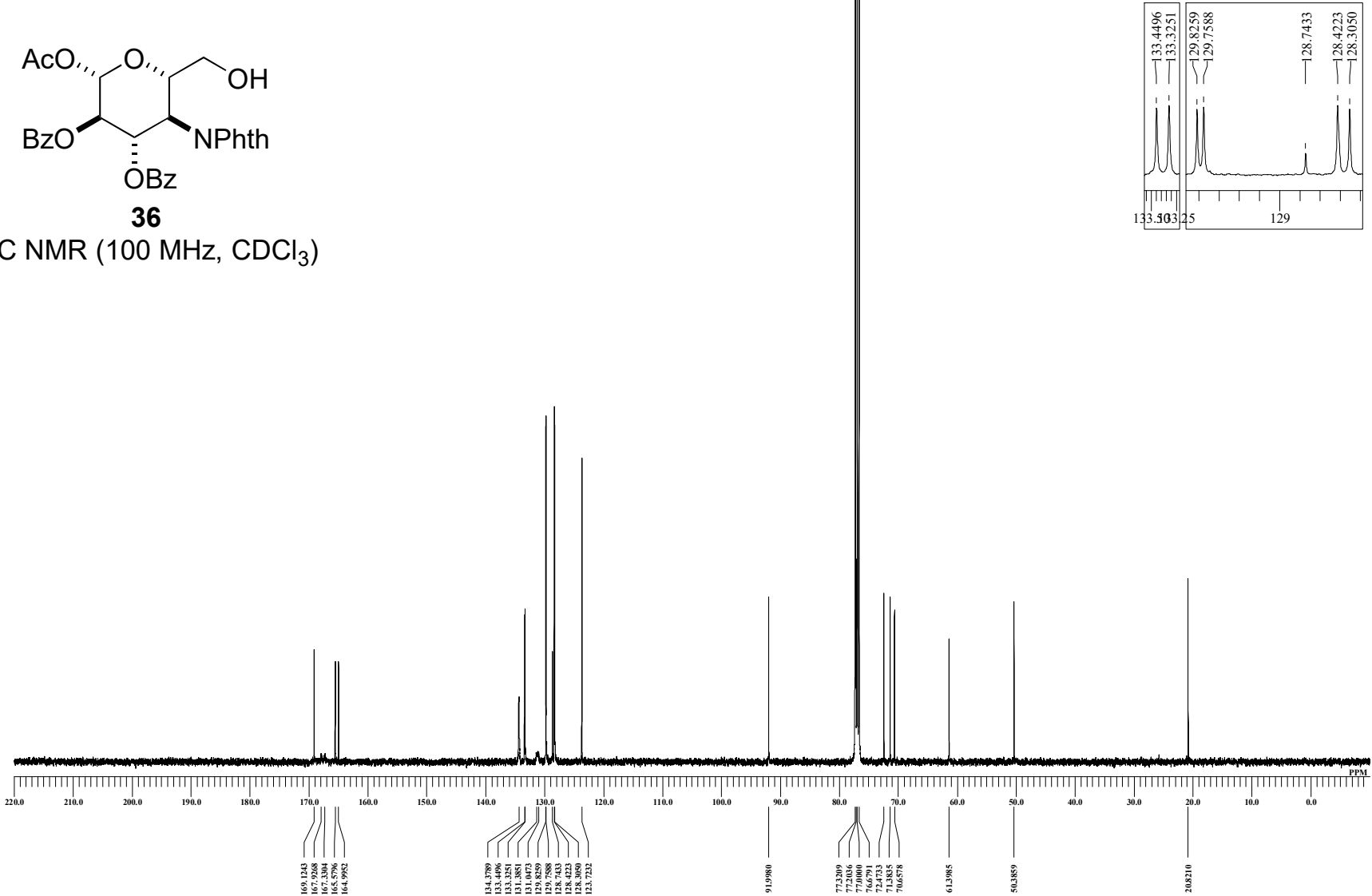


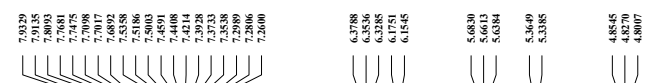

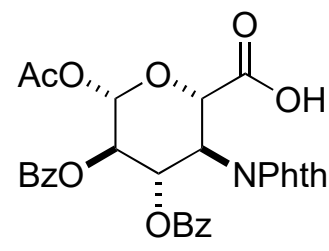

37

${ }^{1} \mathrm{H} \mathrm{NMR}\left(400 \mathrm{MHz}, \mathrm{CDCl}_{3}\right)$

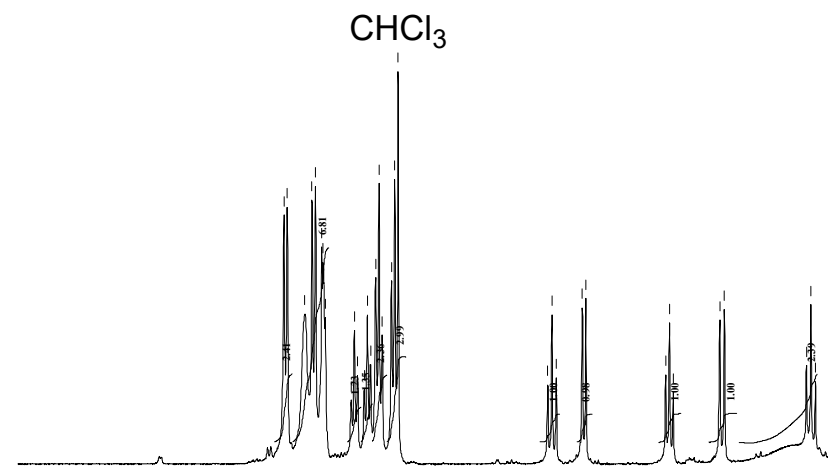

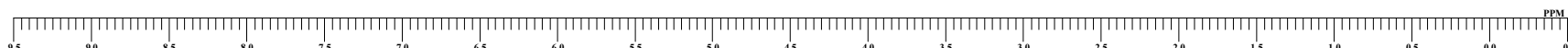

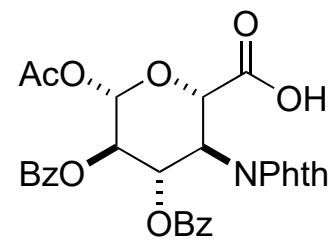

37

${ }^{13} \mathrm{C} \mathrm{NMR}\left(100 \mathrm{MHz}, \mathrm{CDCl}_{3}\right)$
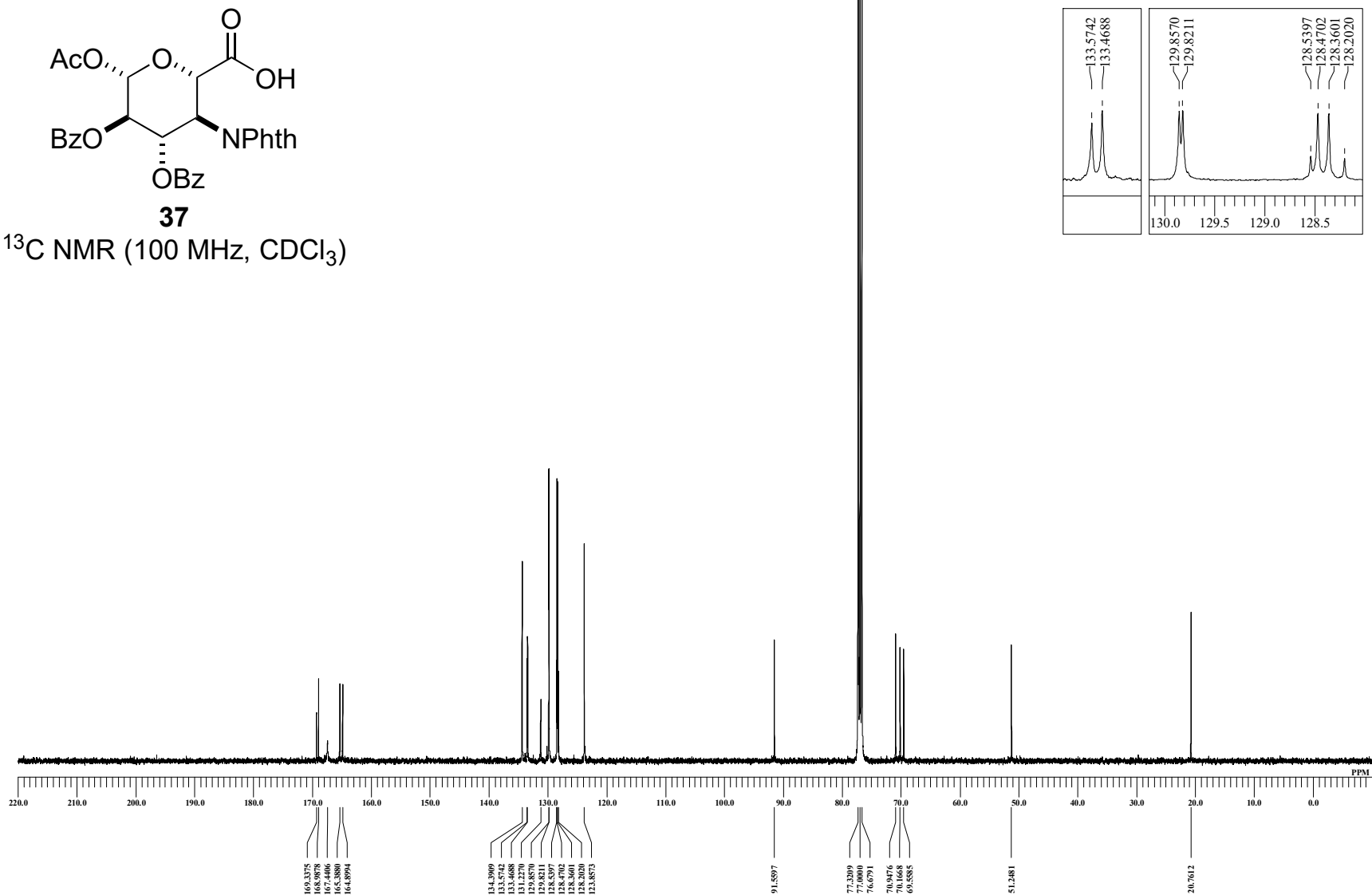


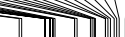

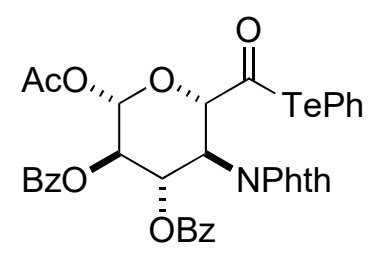

8

${ }^{1} \mathrm{H} \mathrm{NMR}\left(500 \mathrm{MHz}, \mathrm{CDCl}_{3}\right)$

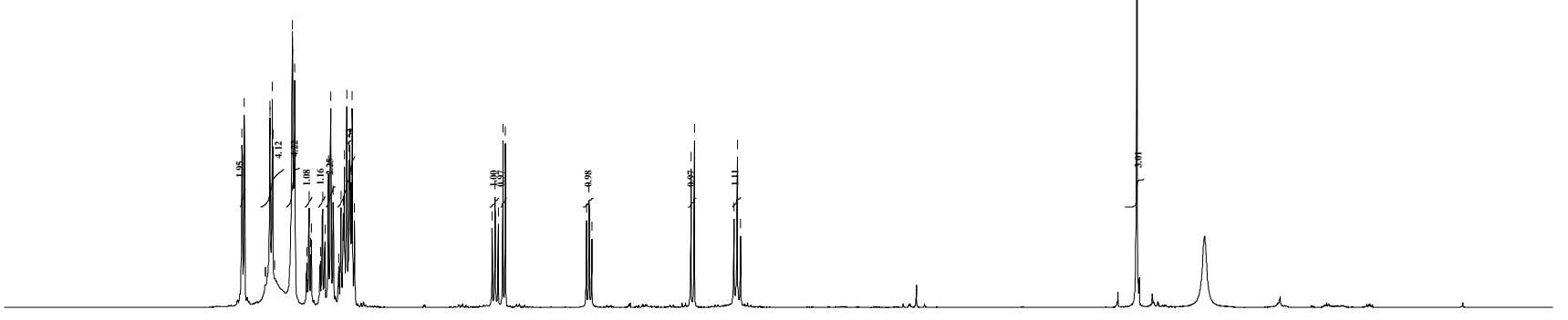

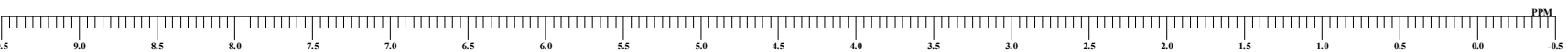

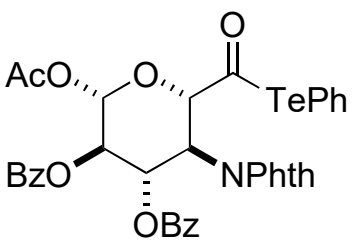

8

${ }^{13} \mathrm{C}$ NMR $\left(125 \mathrm{MHz}, \mathrm{CDCl}_{3}\right)$

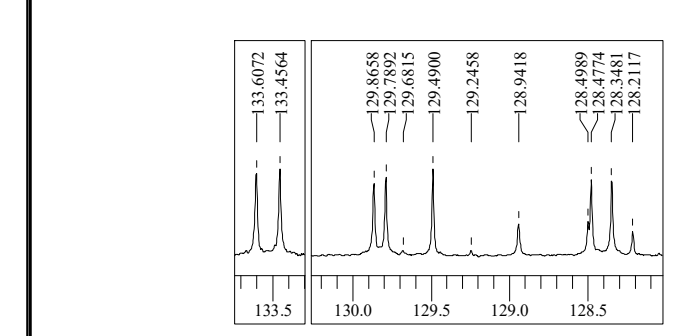

$\mathrm{CDCl}_{3}$

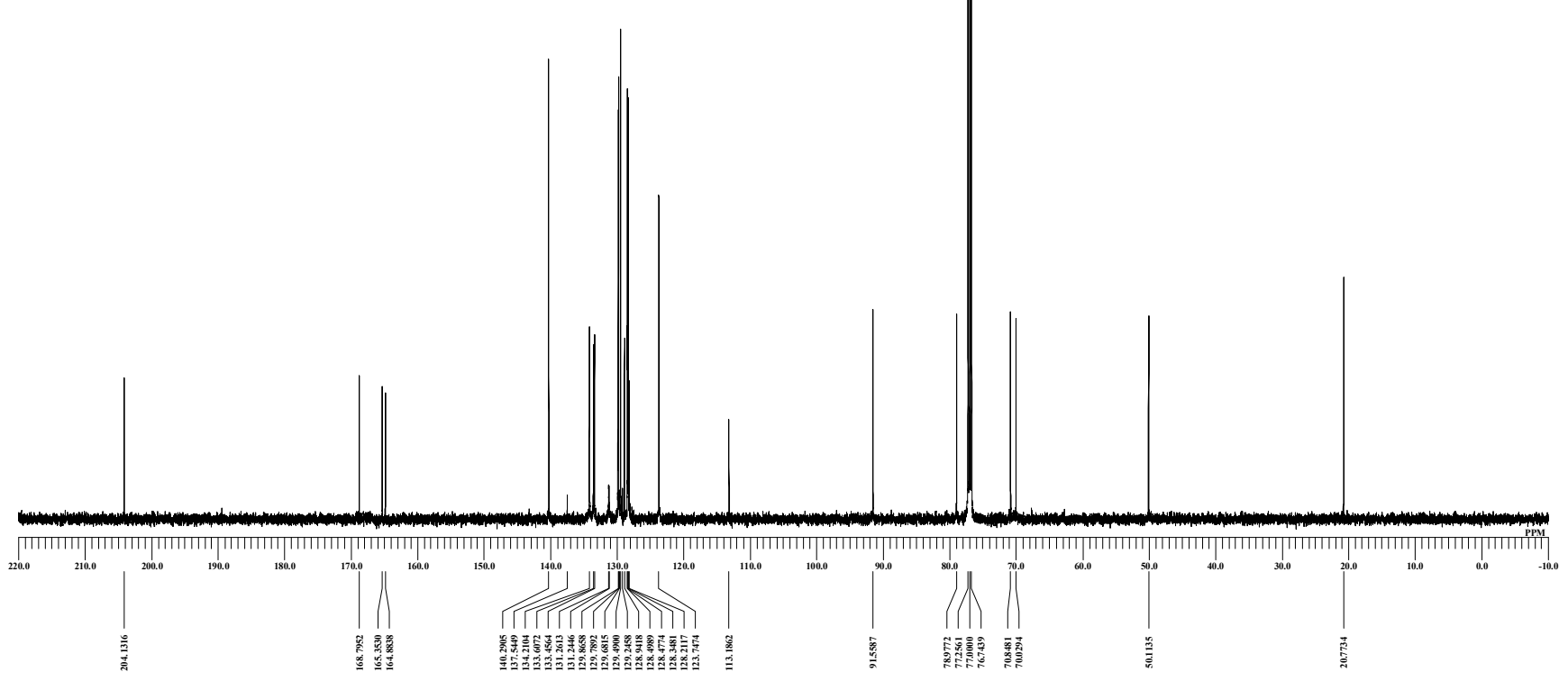




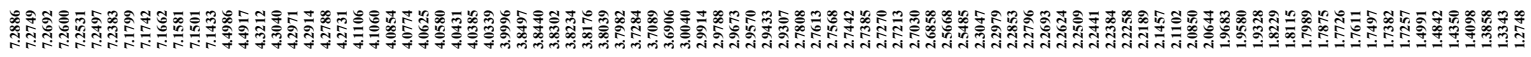

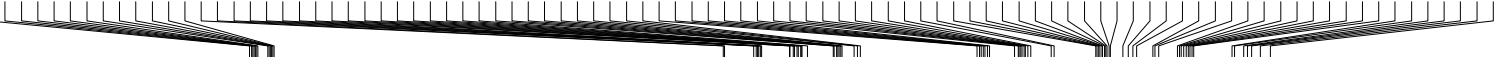<smiles>CC1(C)OC[C@H]2OC3([C@H](O)CCc4ccccc4)OC(C)(C)OC3[C@@H]2O1</smiles>

$21(\mathrm{dr} 2.4: 1)$

${ }^{1} \mathrm{H}$ NMR

$\left(400 \mathrm{MHz}, \mathrm{CDCl}_{3}\right) \quad \mathrm{CHCl}_{3}$

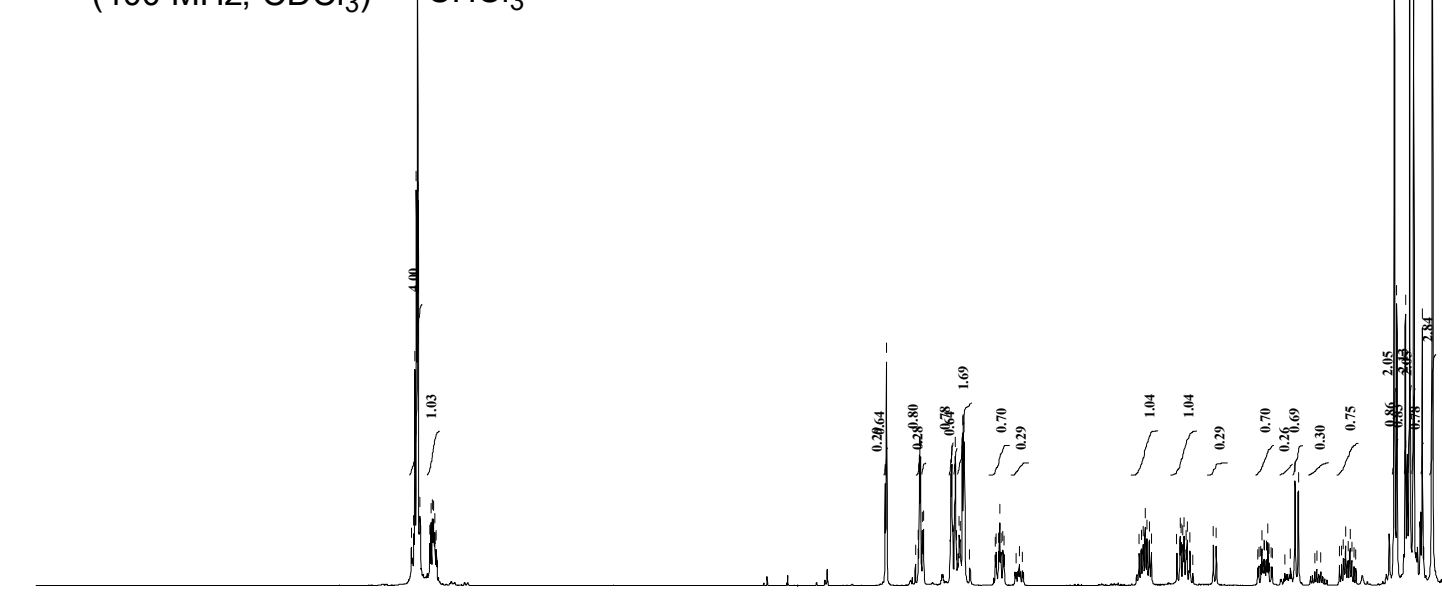

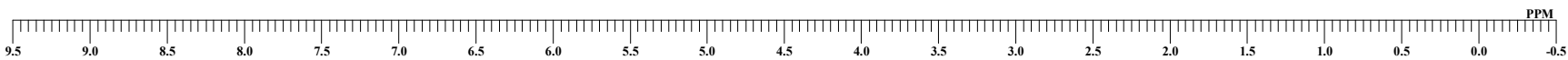<smiles>CC1(C)OC[C@H]2OC3([C@H](O)CCc4ccccc4)OC(C)(C)OC3[C@@H]2O1</smiles>

$21(\mathrm{dr} 2.4: 1)$

${ }^{13} \mathrm{C}$ NMR $\left(100 \mathrm{MHz}, \mathrm{CDCl}_{3}\right)$

$\mathrm{CDCl}_{3}$
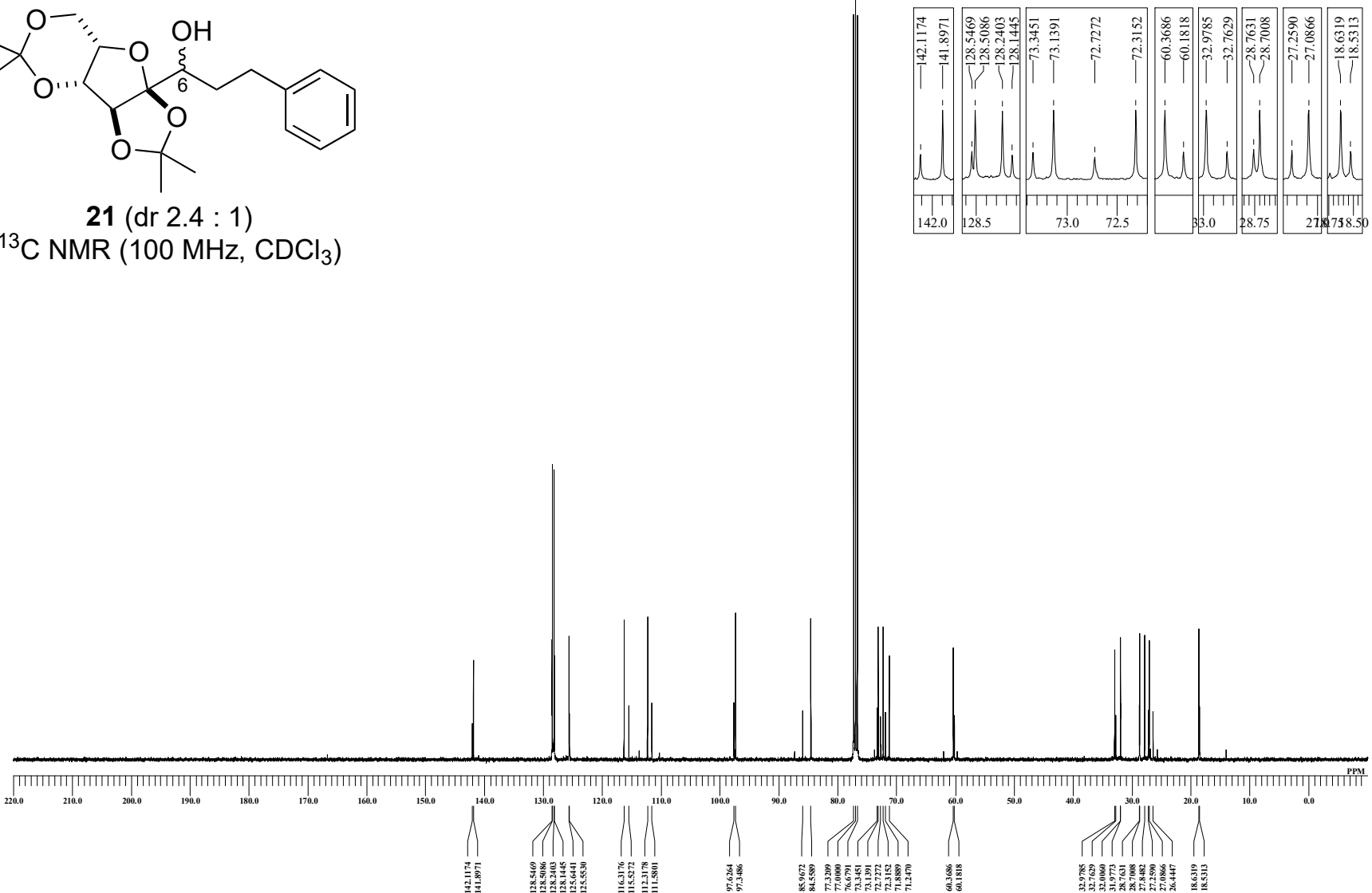


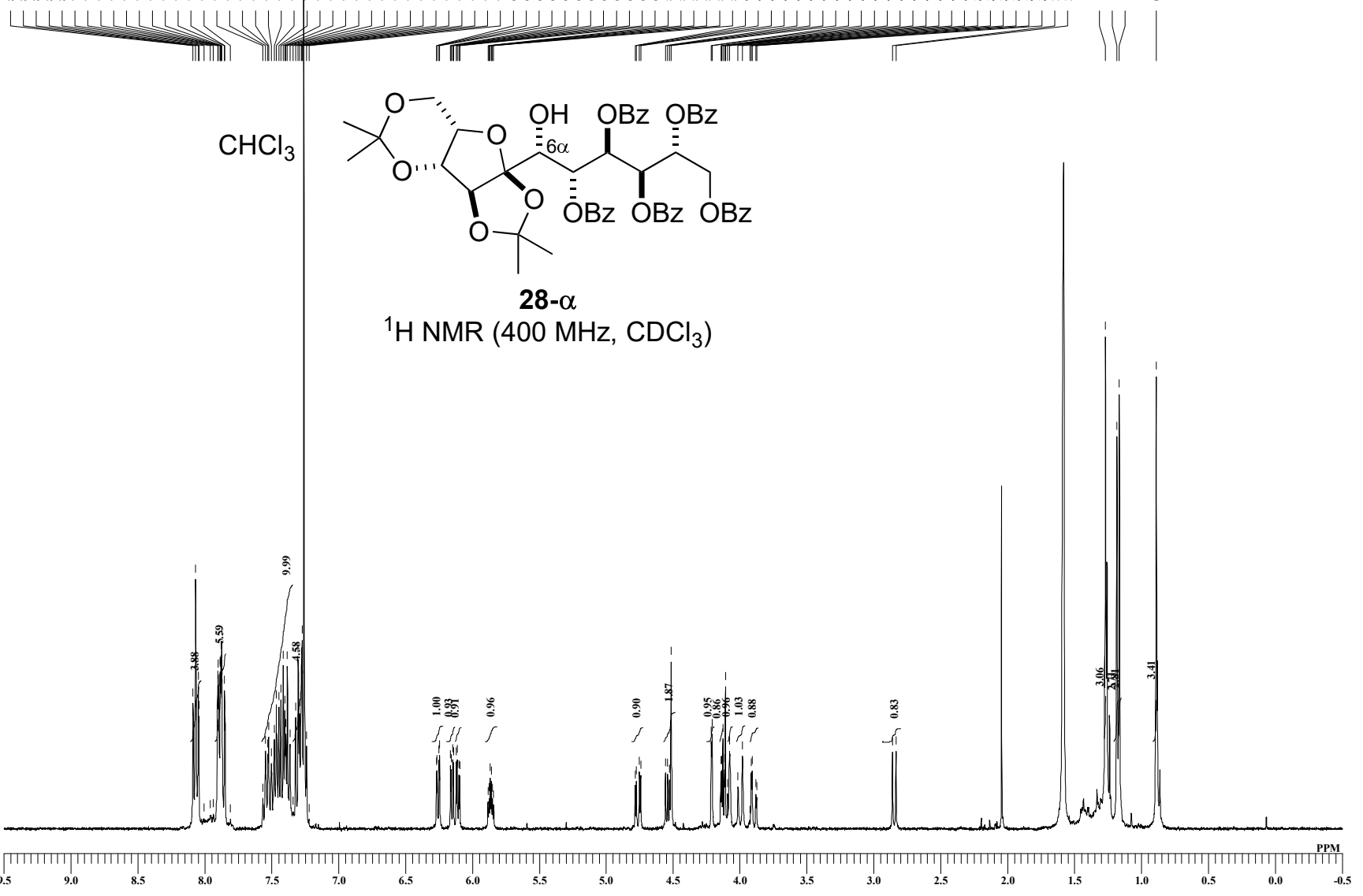

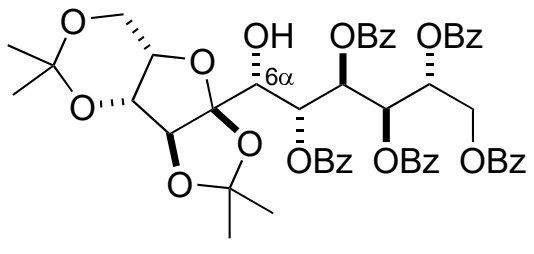

28- $\alpha$

${ }^{13} \mathrm{C}$ NMR $\left(125 \mathrm{MHz}, \mathrm{CDCl}_{3}\right)$
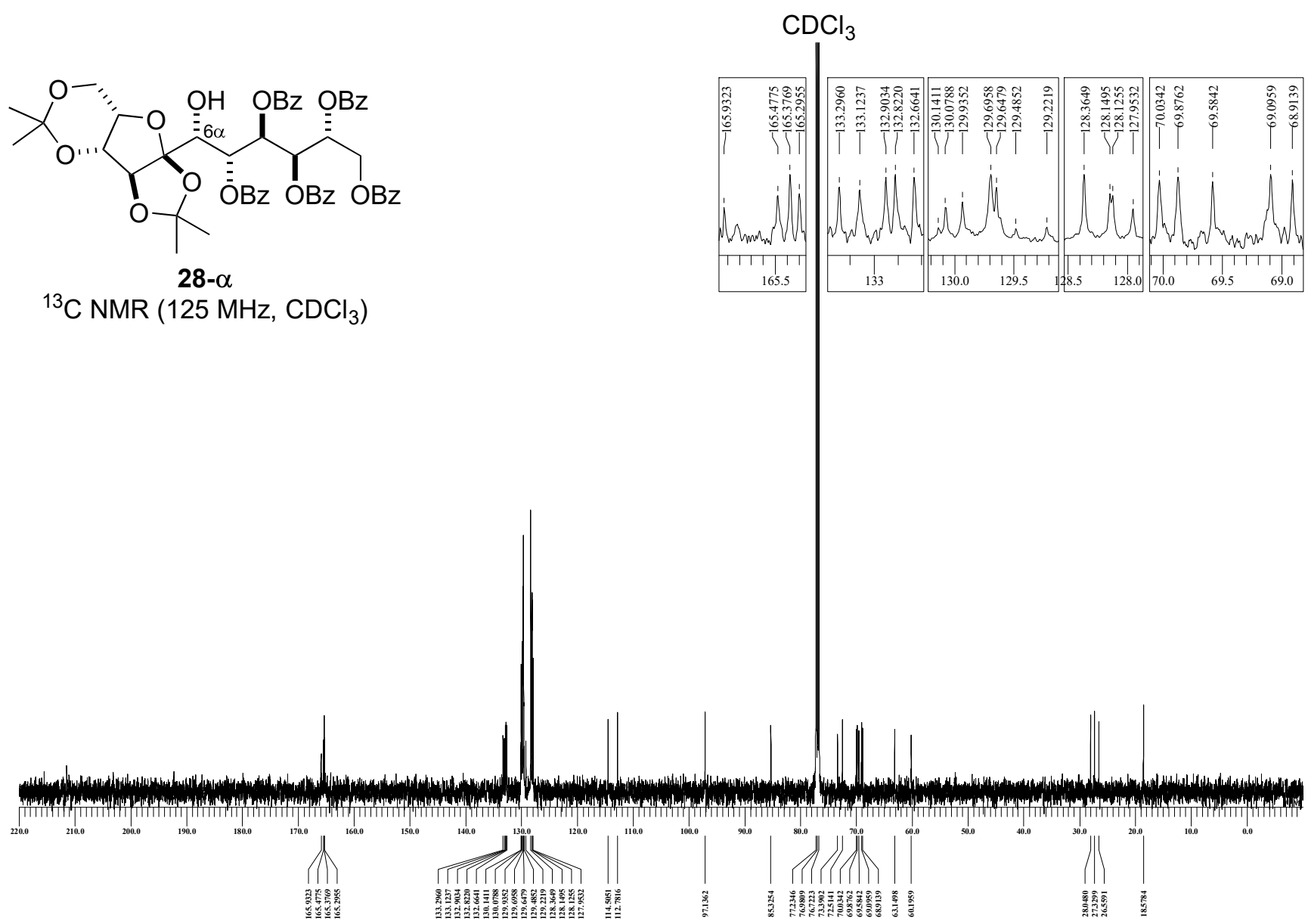

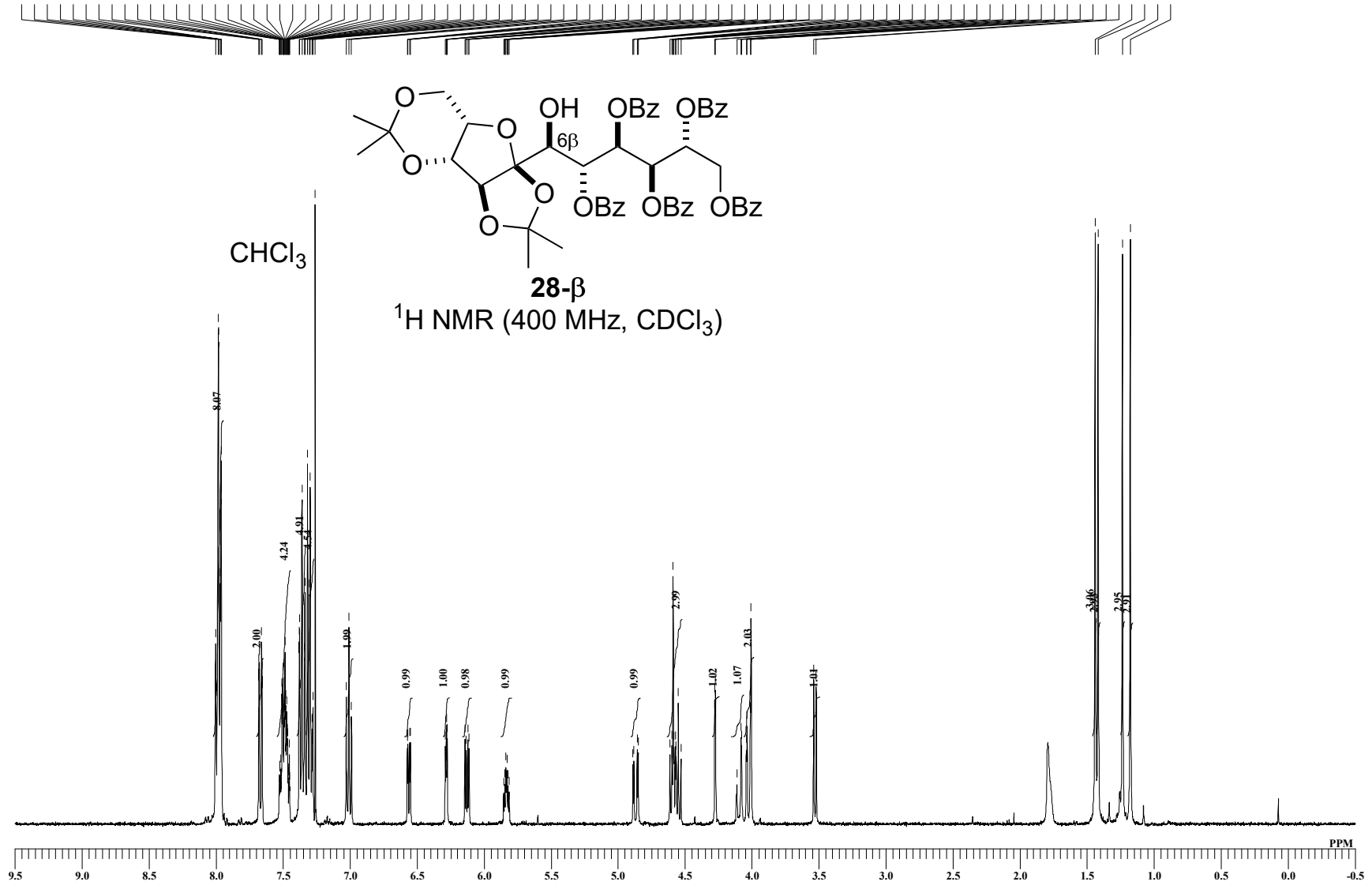<smiles>CC(C)(C)OC(=O)c1ccccc1</smiles>

28- $\beta$

${ }^{13} \mathrm{C} \mathrm{NMR}\left(100 \mathrm{MHz}, \mathrm{CDCl}_{3}\right)$

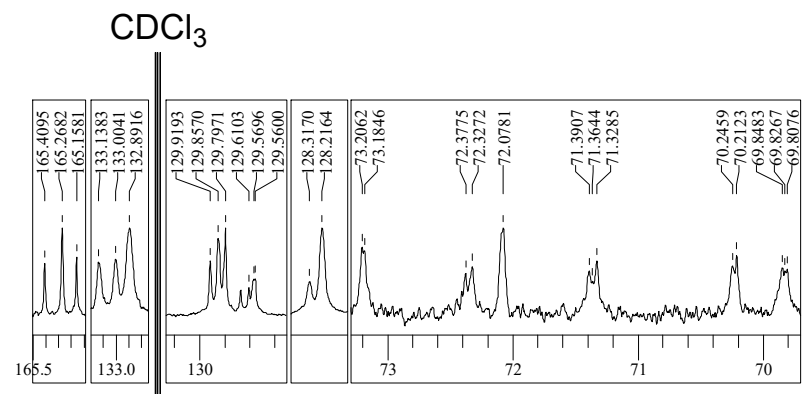

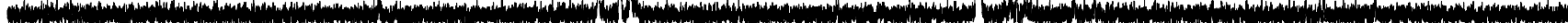

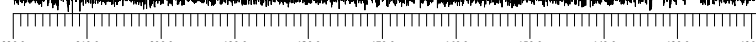

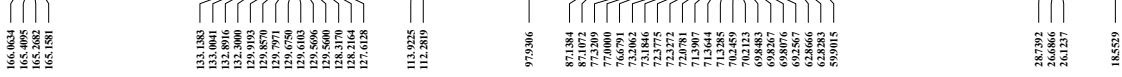




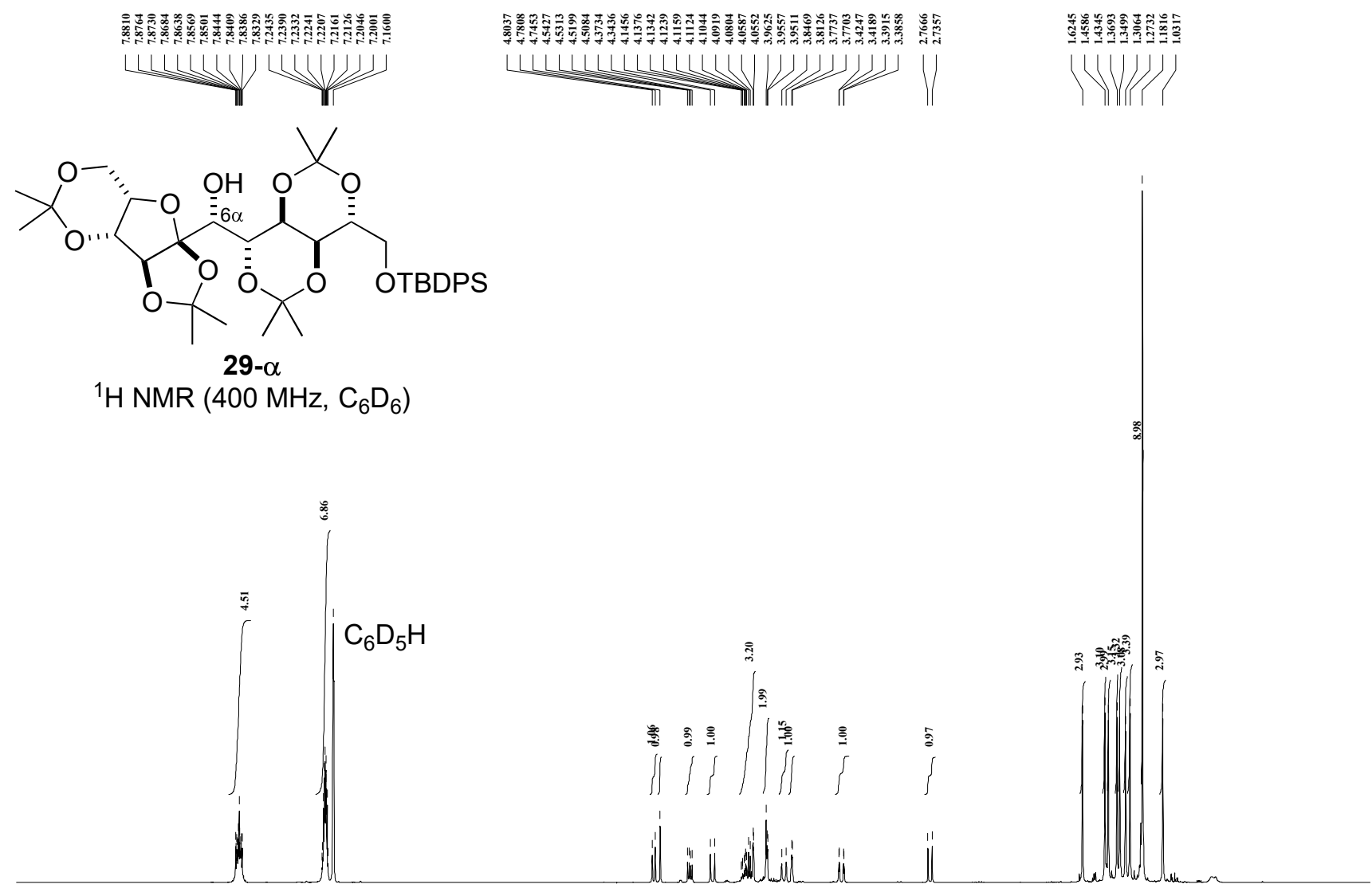

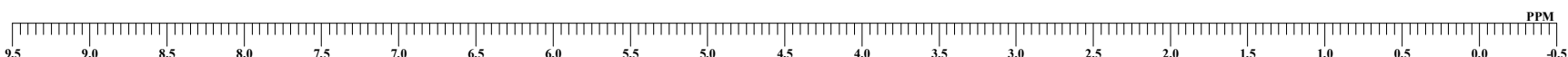

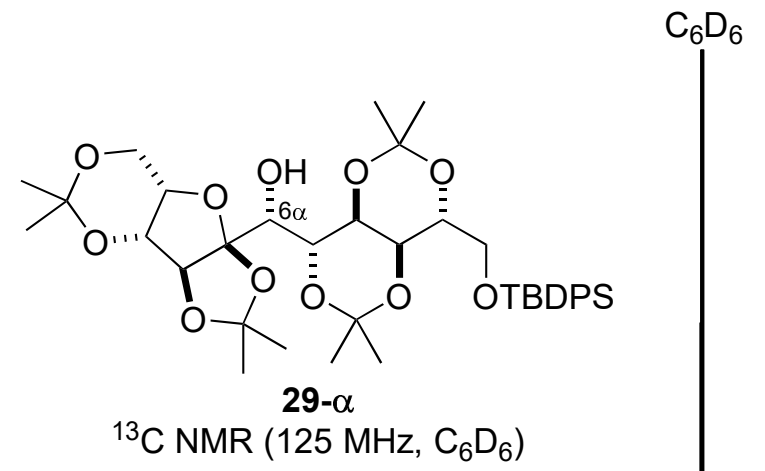

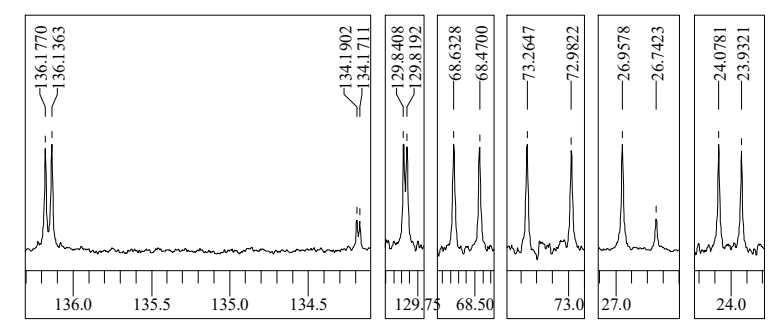

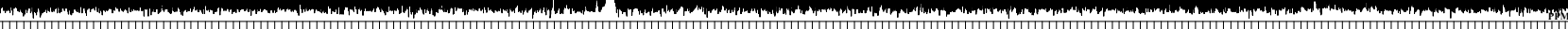

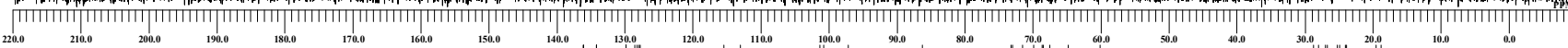

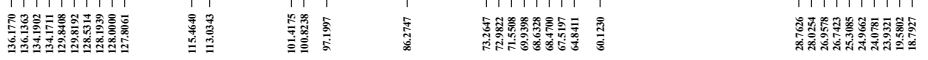




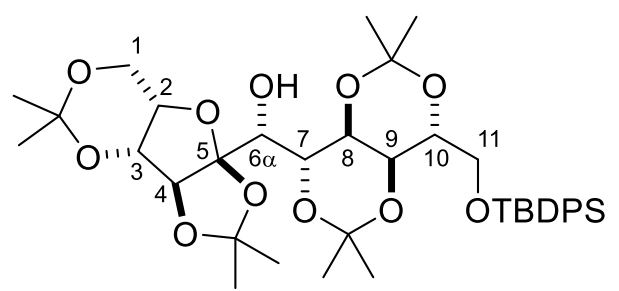

29- $\alpha$

DQF-COSY $\left(500 \mathrm{MHz}, \mathrm{C}_{6} \mathrm{D}_{6}\right)$

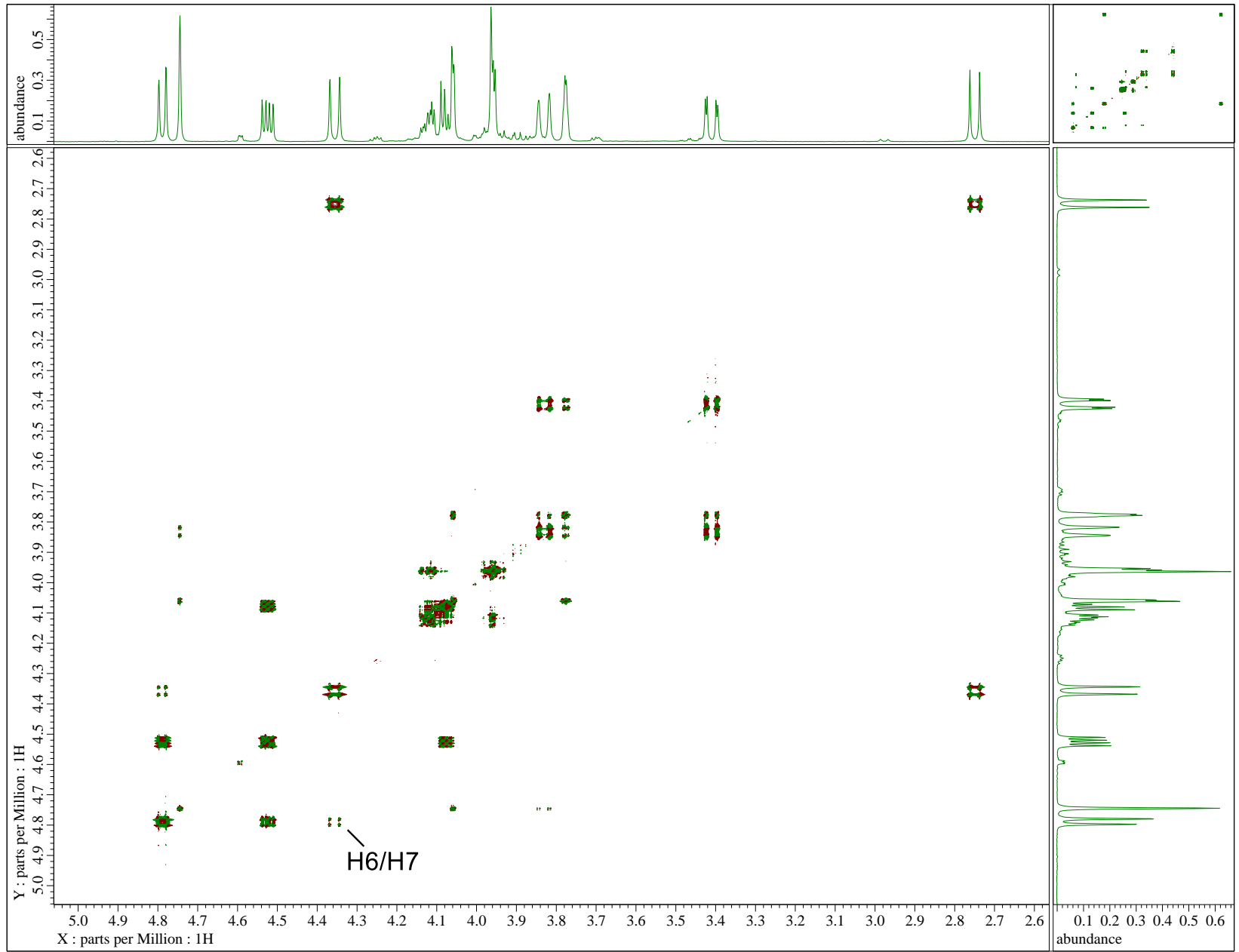


HMQC $\left(500 \mathrm{MHz}, \mathrm{C}_{6} \mathrm{D}_{6}\right)$

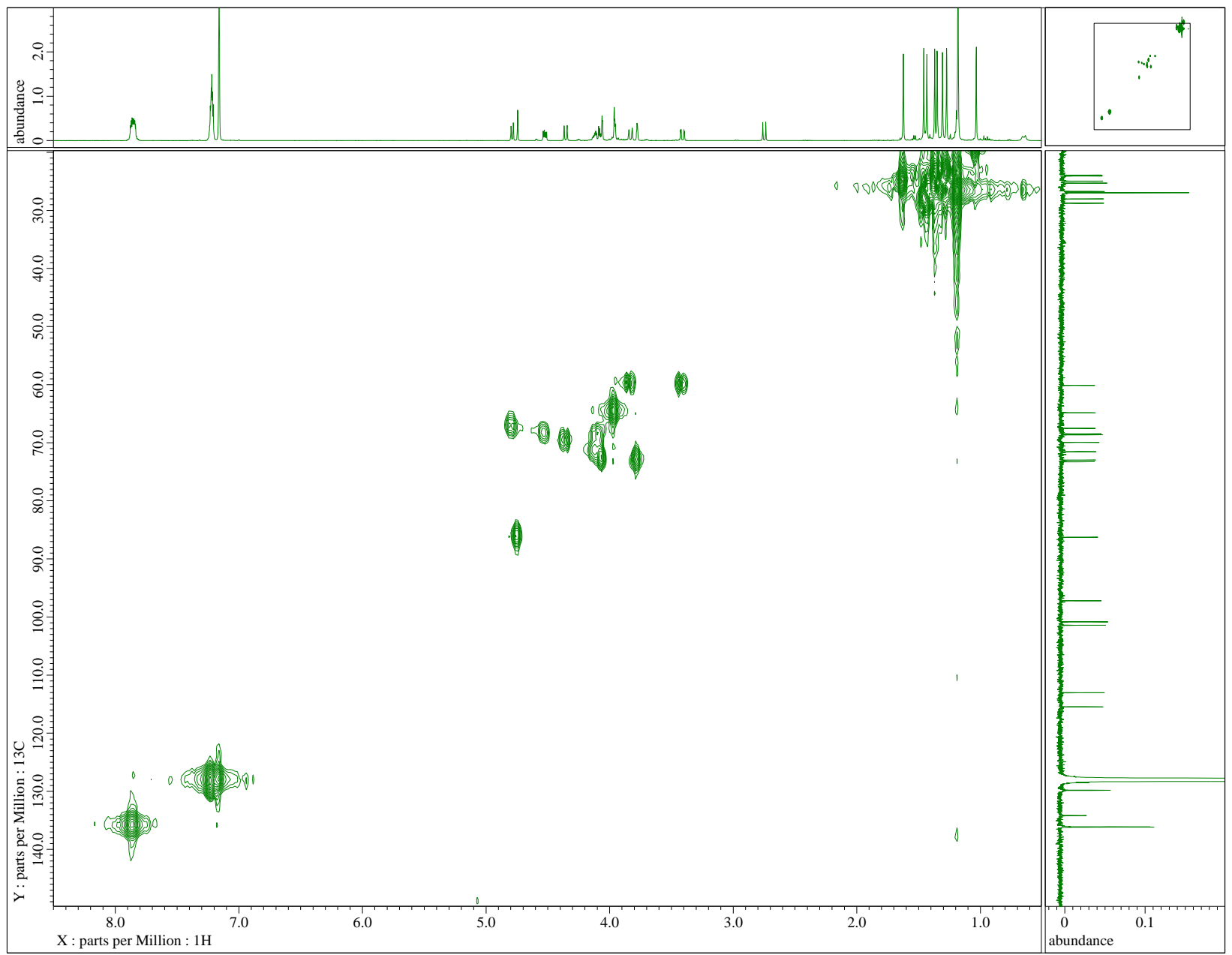


J-resolved HMBC-1 $\left(500 \mathrm{MHz}, \mathrm{C}_{6} \mathrm{D}_{6}\right)$
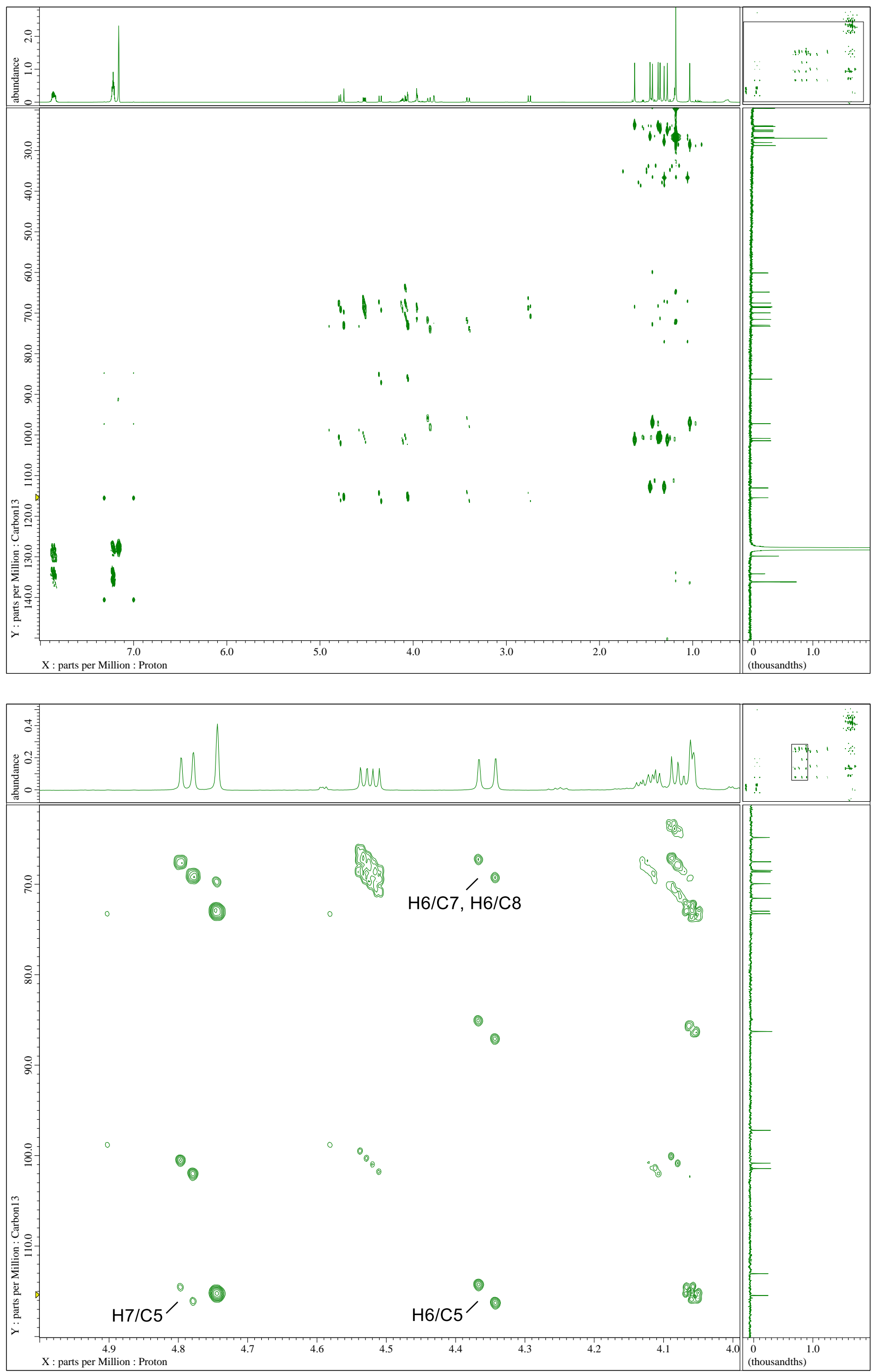
NOESY $\left(500 \mathrm{MHz}, \mathrm{C}_{6} \mathrm{D}_{6}\right)$
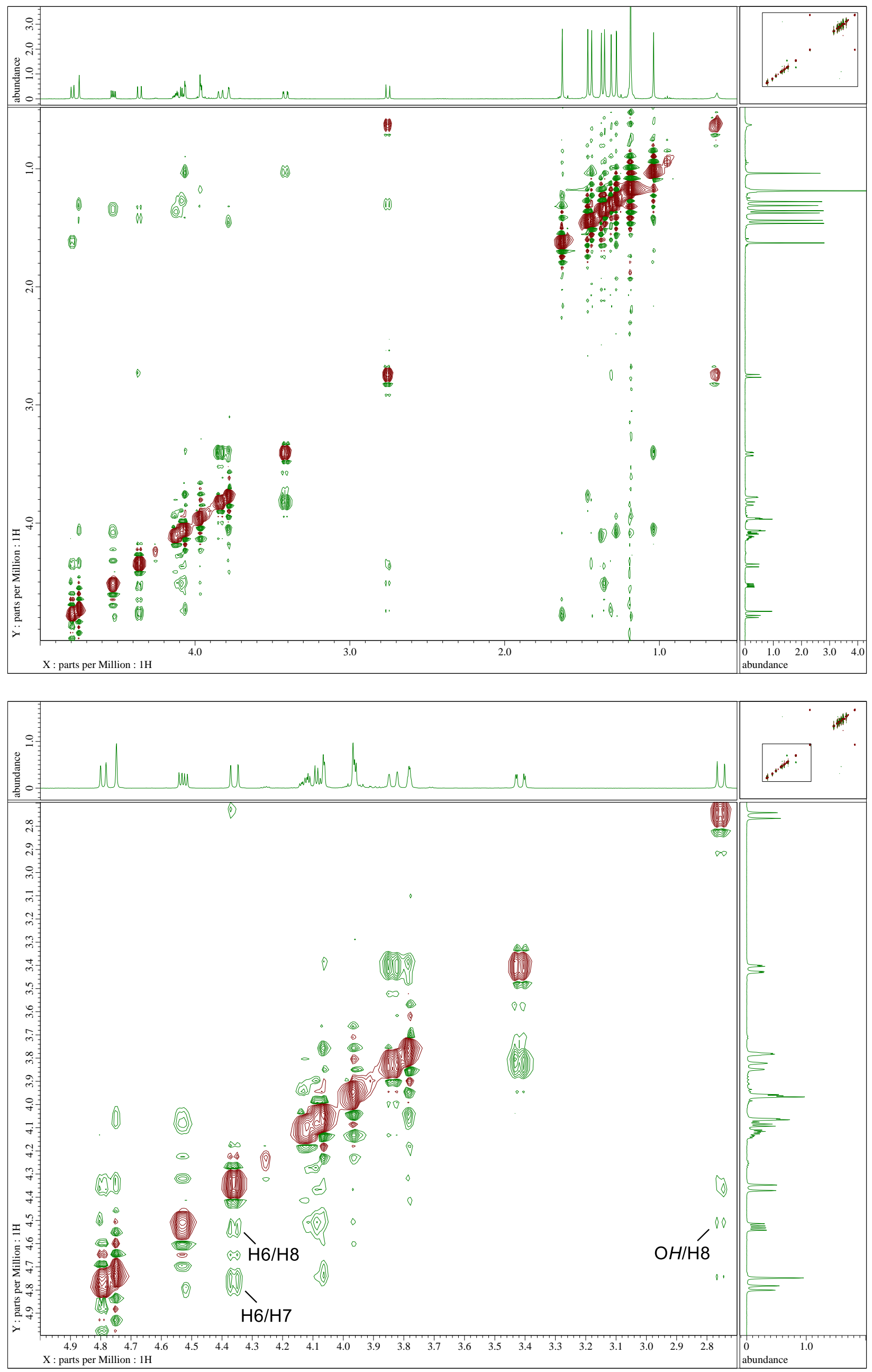

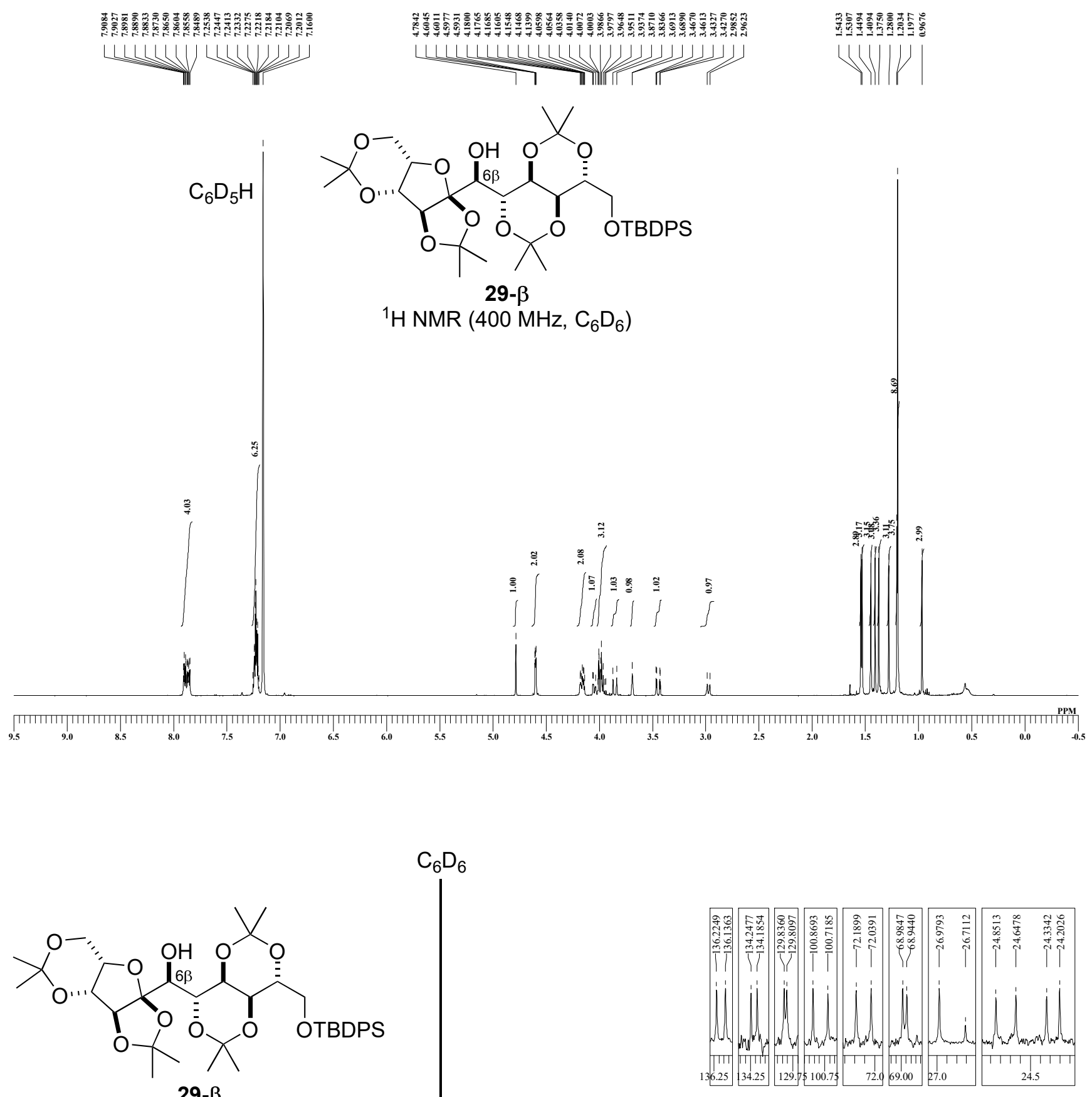

${ }^{13} \mathrm{C}$ NMR $\left(125 \mathrm{MHz}, \mathrm{C}_{6} \mathrm{D}_{6}\right)$

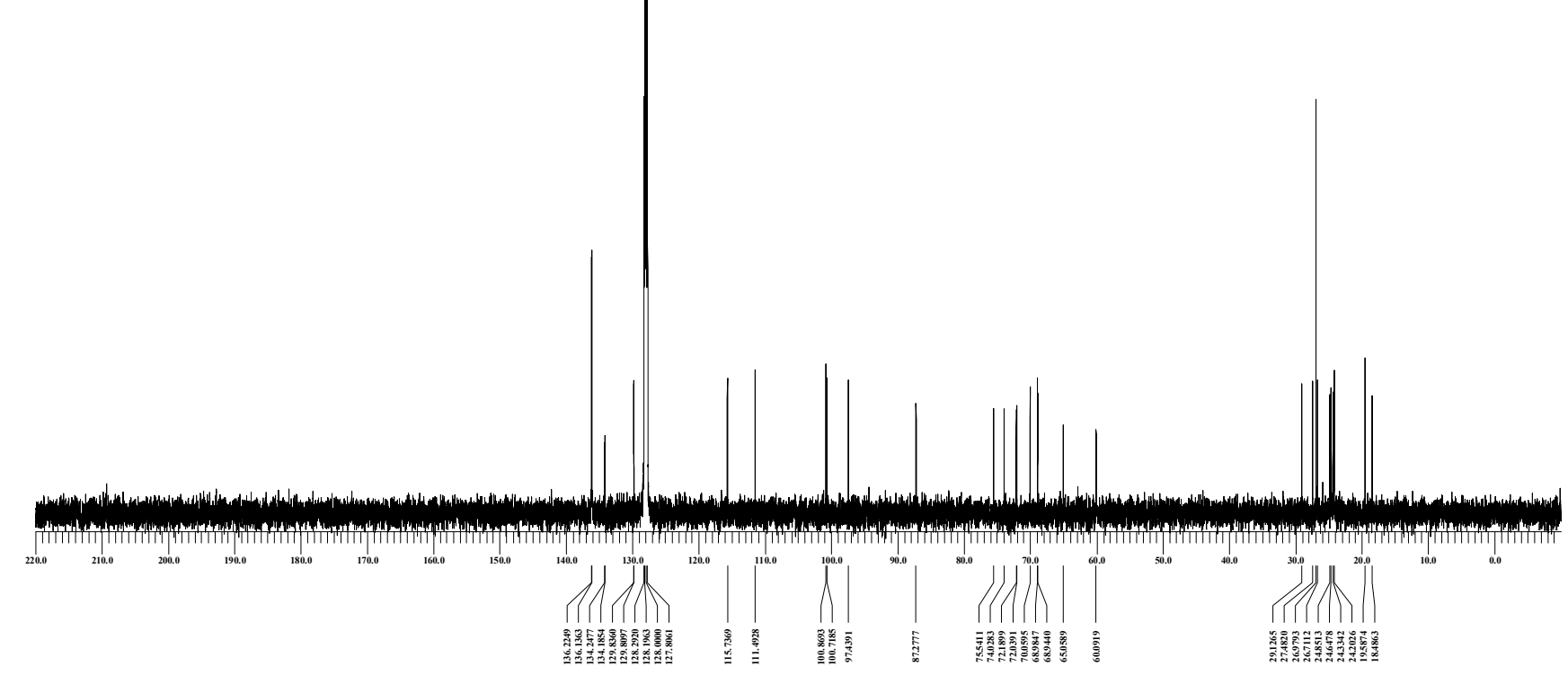



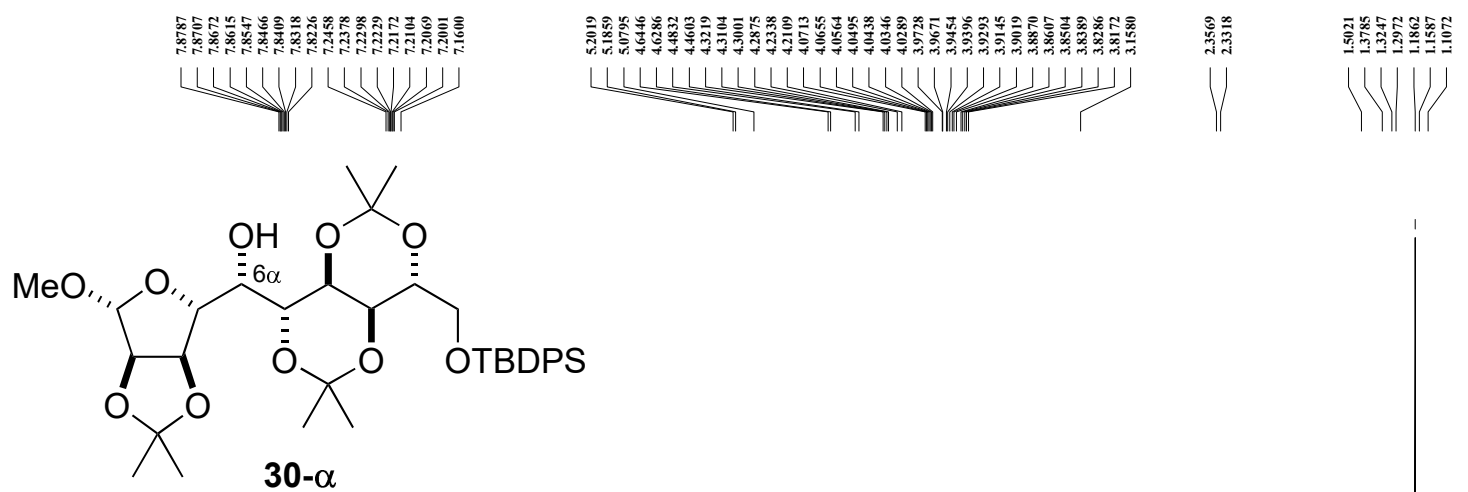

${ }^{1} \mathrm{H}$ NMR $\left(400 \mathrm{MHz}, \mathrm{C}_{6} \mathrm{D}_{6}\right)$

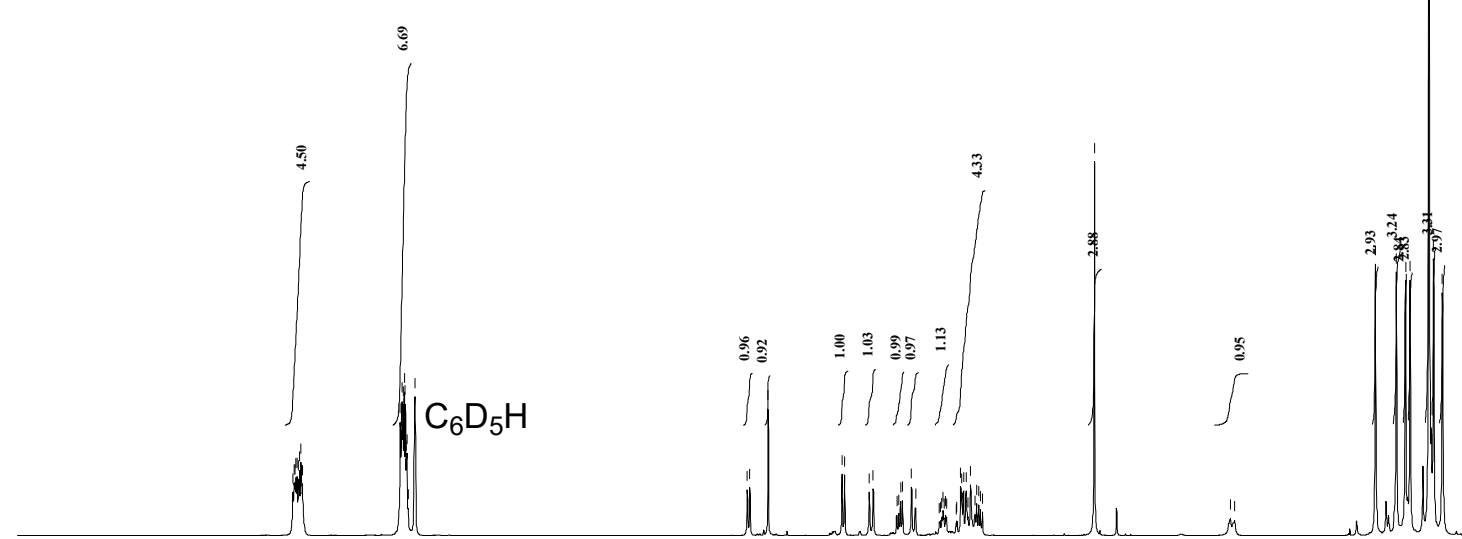

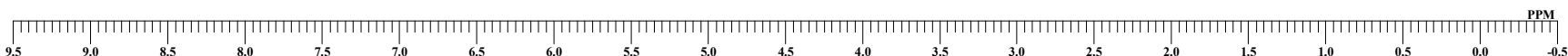

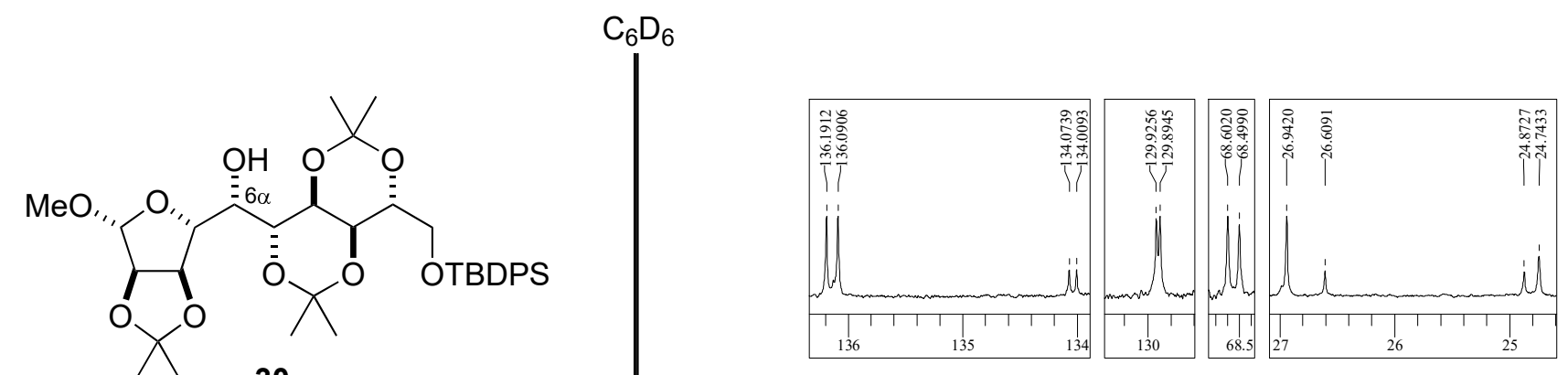

${ }^{13} \mathrm{C}$ NMR $\left(100 \mathrm{MHz}, \mathrm{C}_{6} \mathrm{D}_{6}\right)$

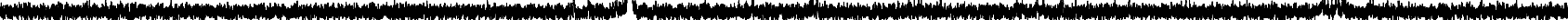

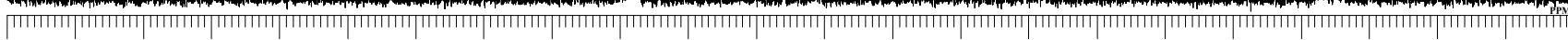

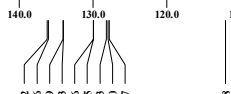




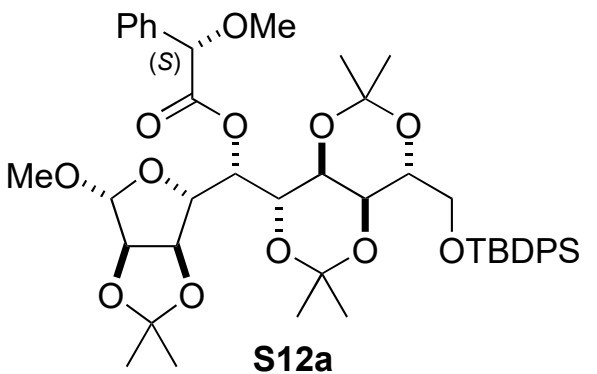

${ }^{1} \mathrm{H}$ NMR $\left(500 \mathrm{MHz}, \mathrm{CDCl}_{3}\right)$

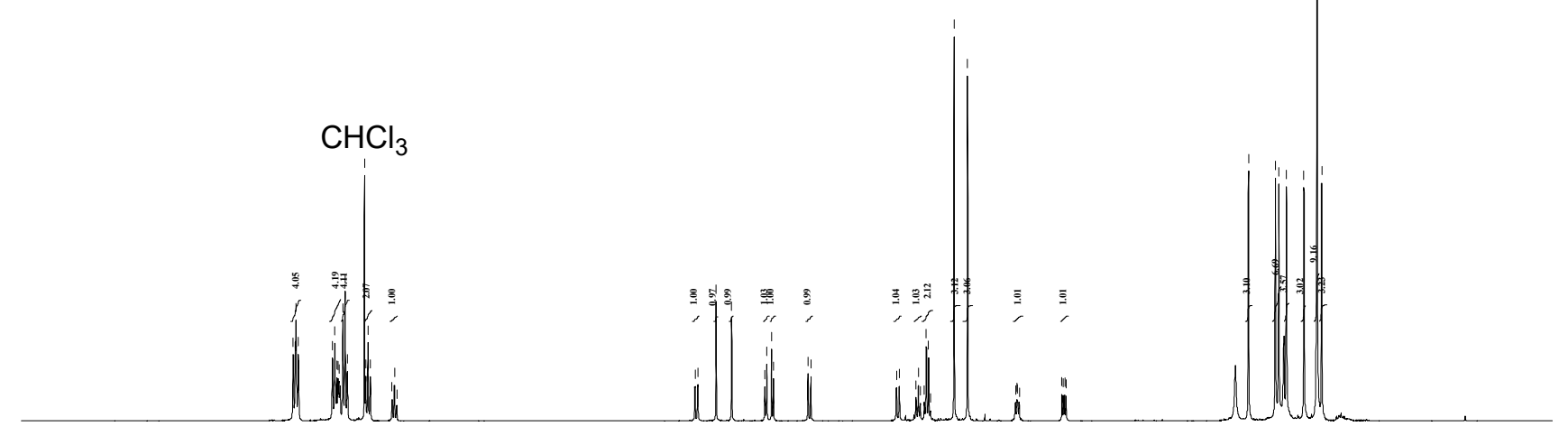

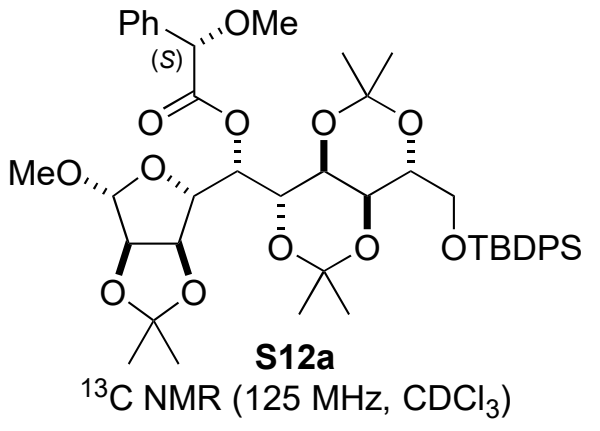

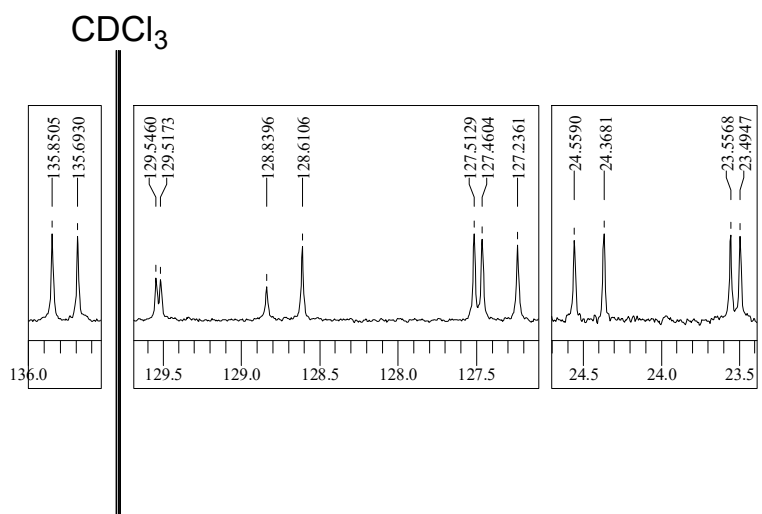

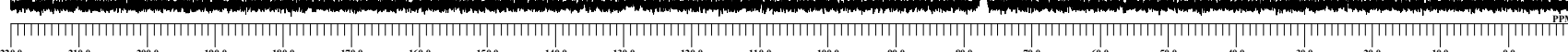

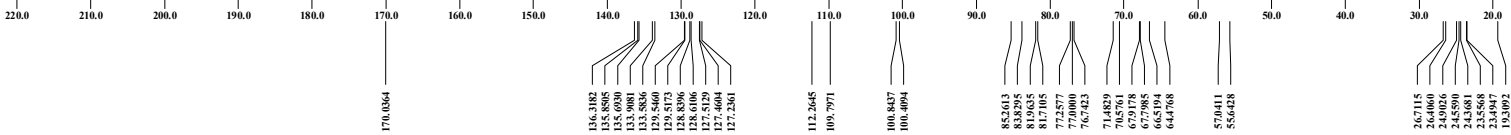




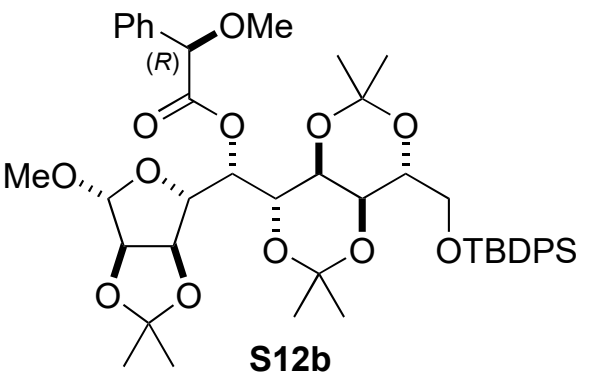

S12b

${ }^{1} \mathrm{H}$ NMR (500 MHz, $\left.\mathrm{CDCl}_{3}\right)$

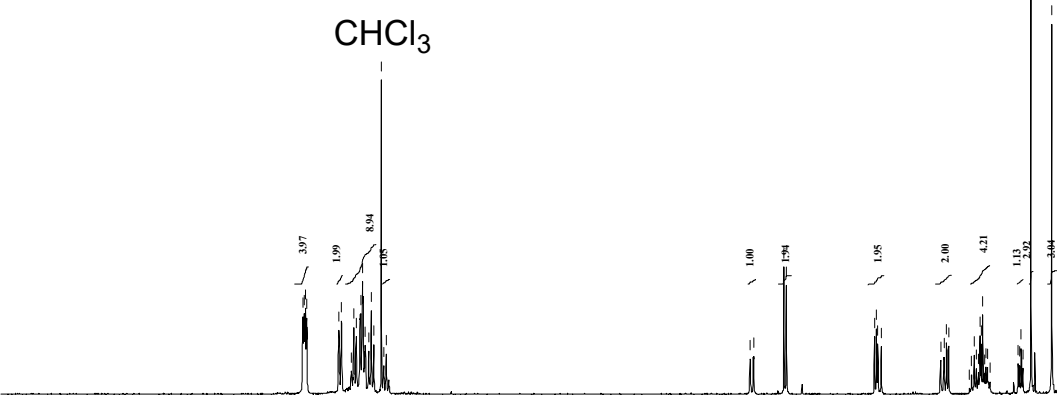

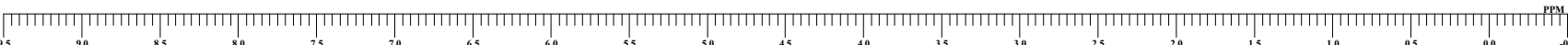

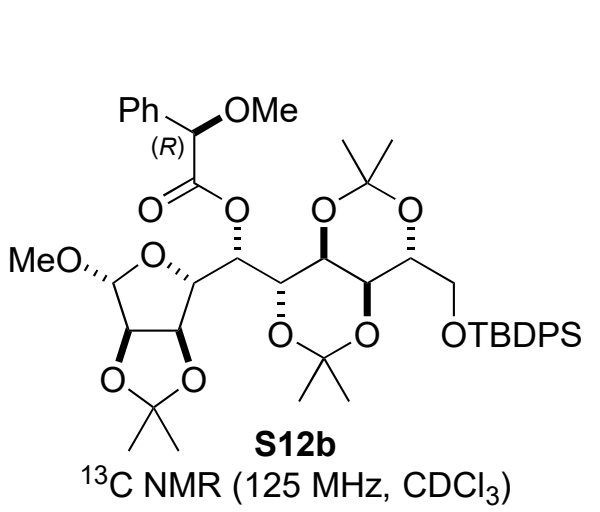

$\mathrm{CDCl}_{3}$

$\mathrm{Ph}$

O

${ }^{13} \mathrm{C} \mathrm{NMR}\left(125 \mathrm{MHz}, \mathrm{CDCl}_{3}\right)$

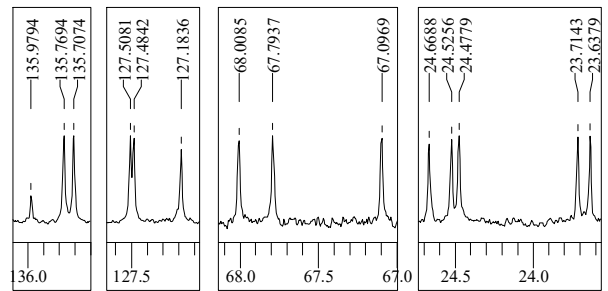

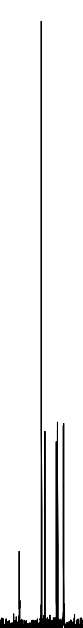

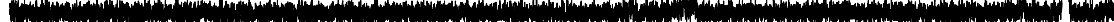

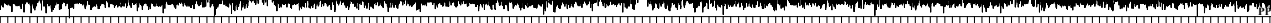

220.0210 .0
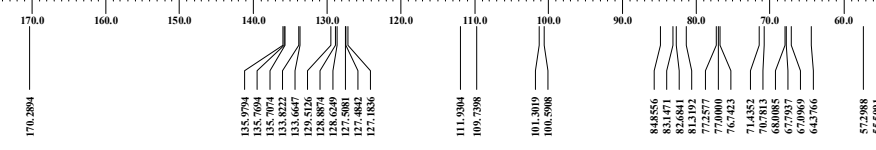

$$
\begin{aligned}
& 1 \\
& \text { 2. }
\end{aligned}
$$




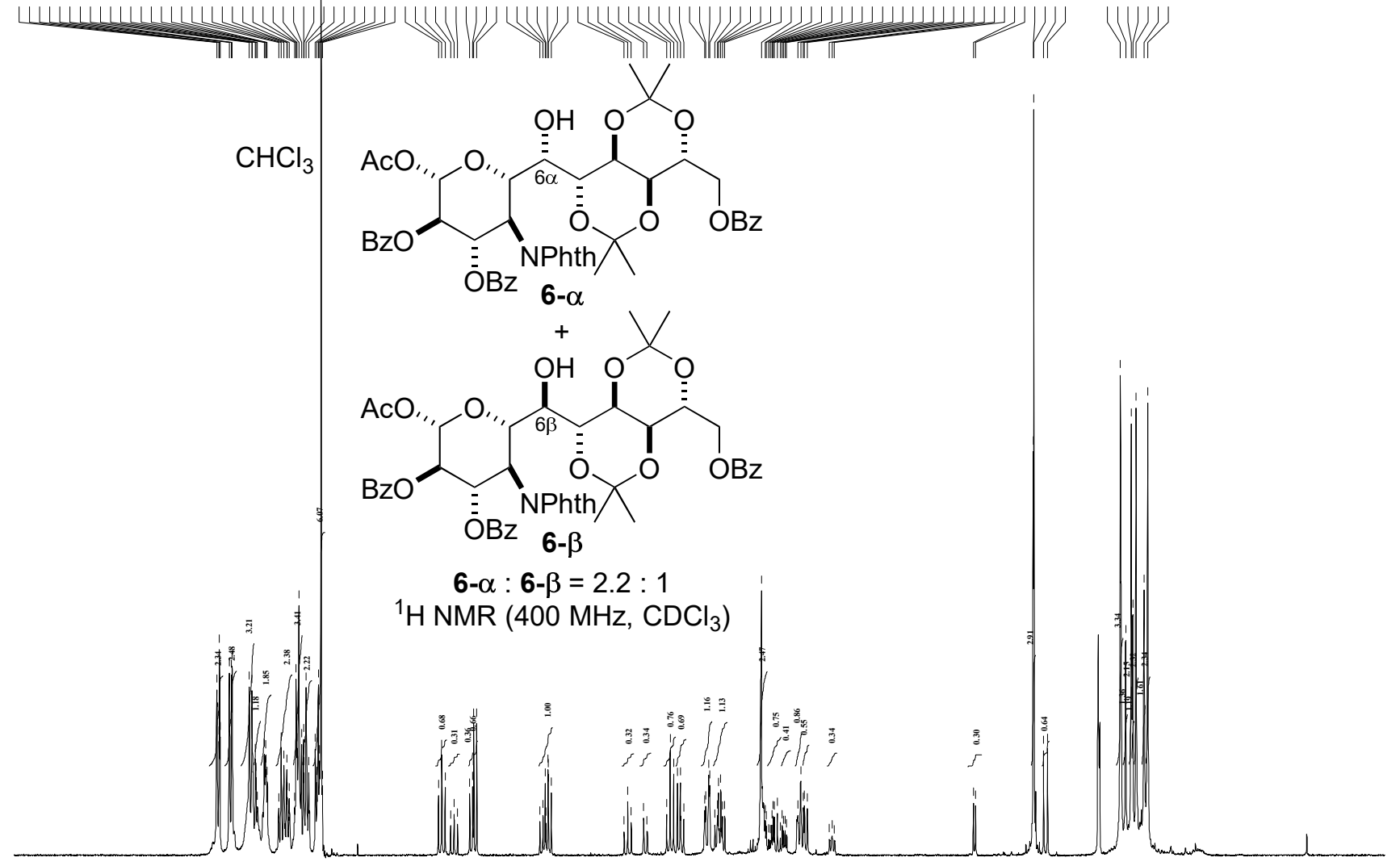

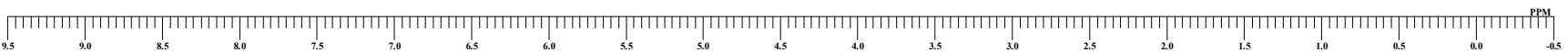



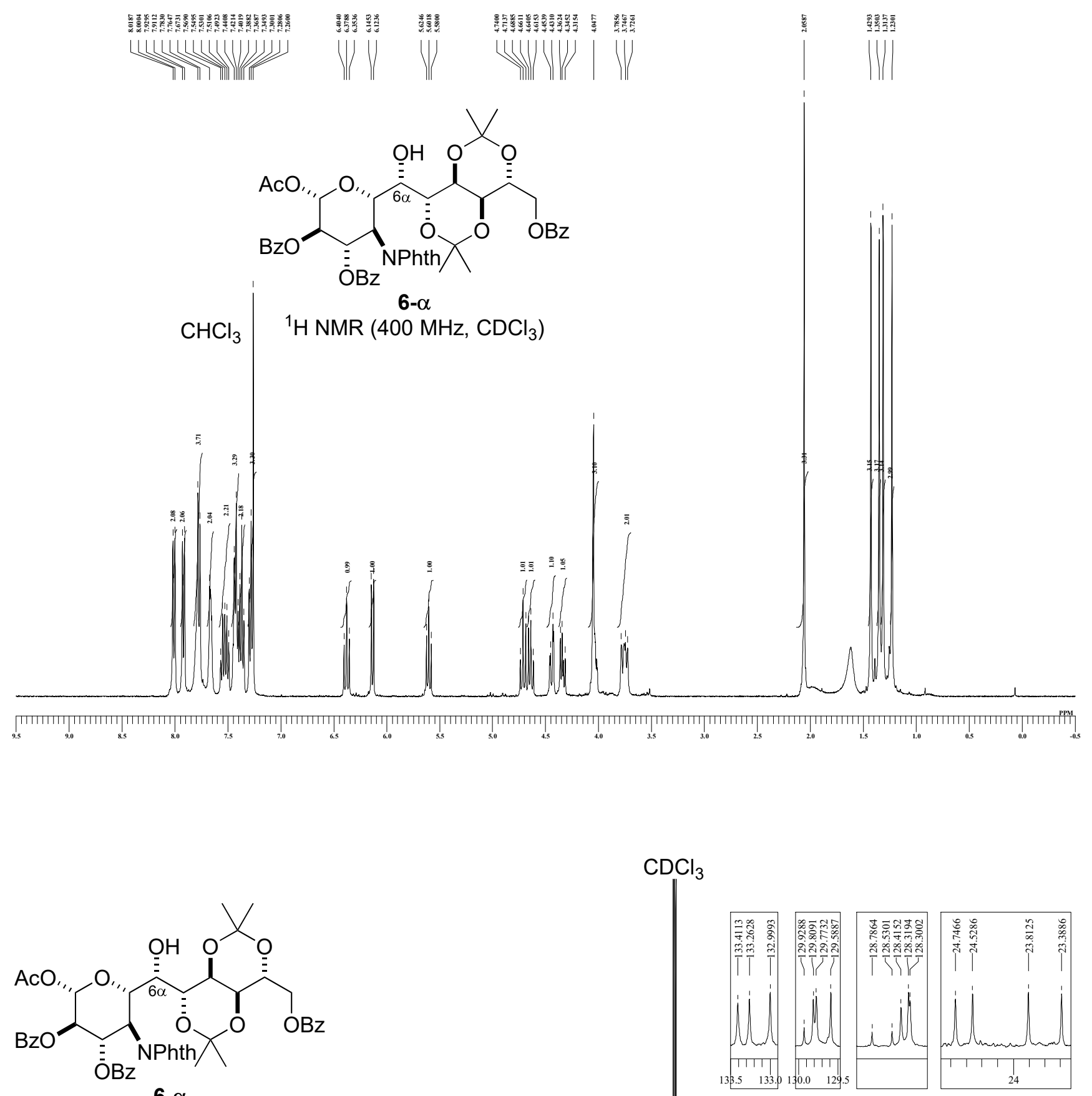

${ }^{13} \mathrm{C}$ NMR $\left(100 \mathrm{MHz}, \mathrm{CDCl}_{3}\right)$

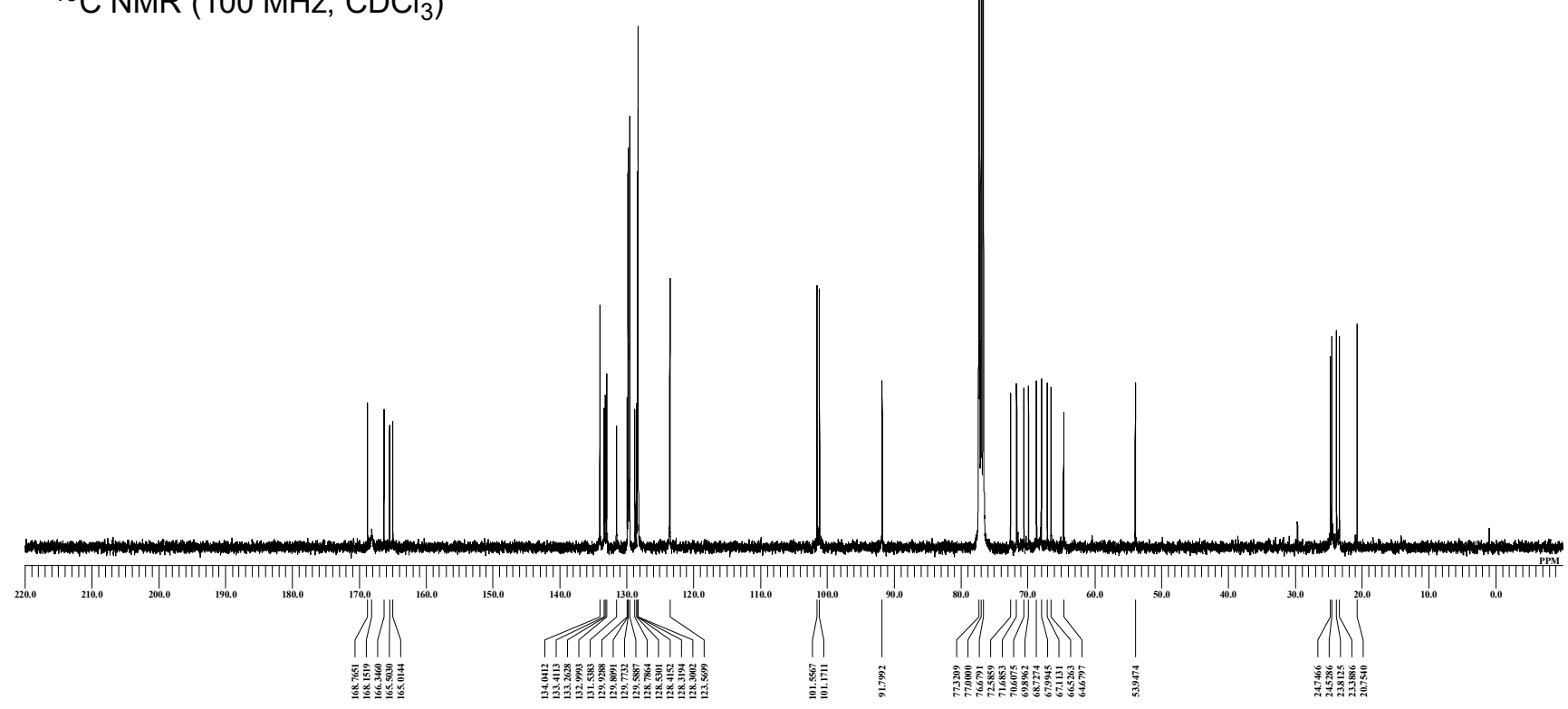



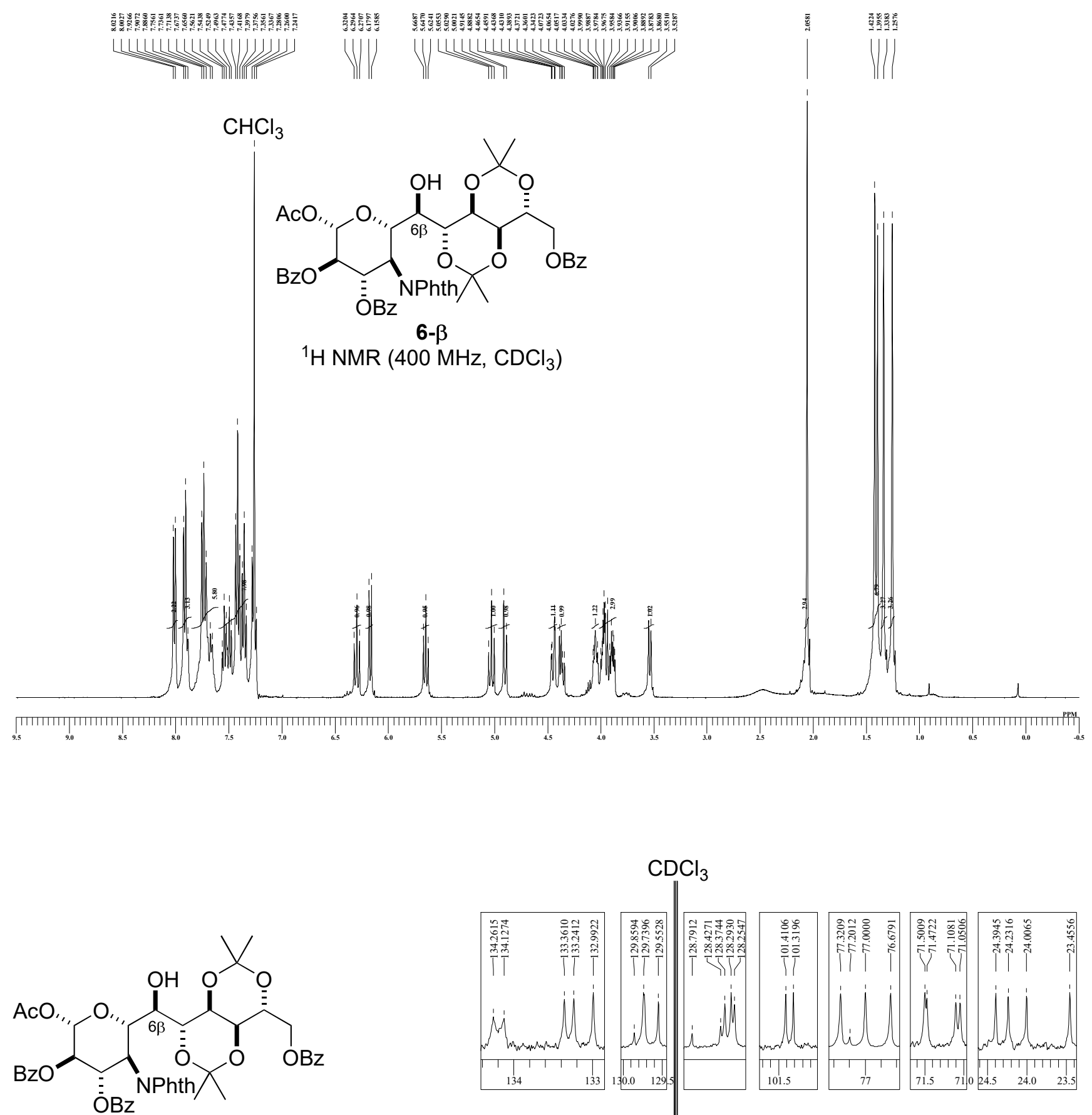

${ }^{13} \mathrm{C}$ NMR $\left(100 \mathrm{MHz}, \mathrm{CDCl}_{3}\right)$

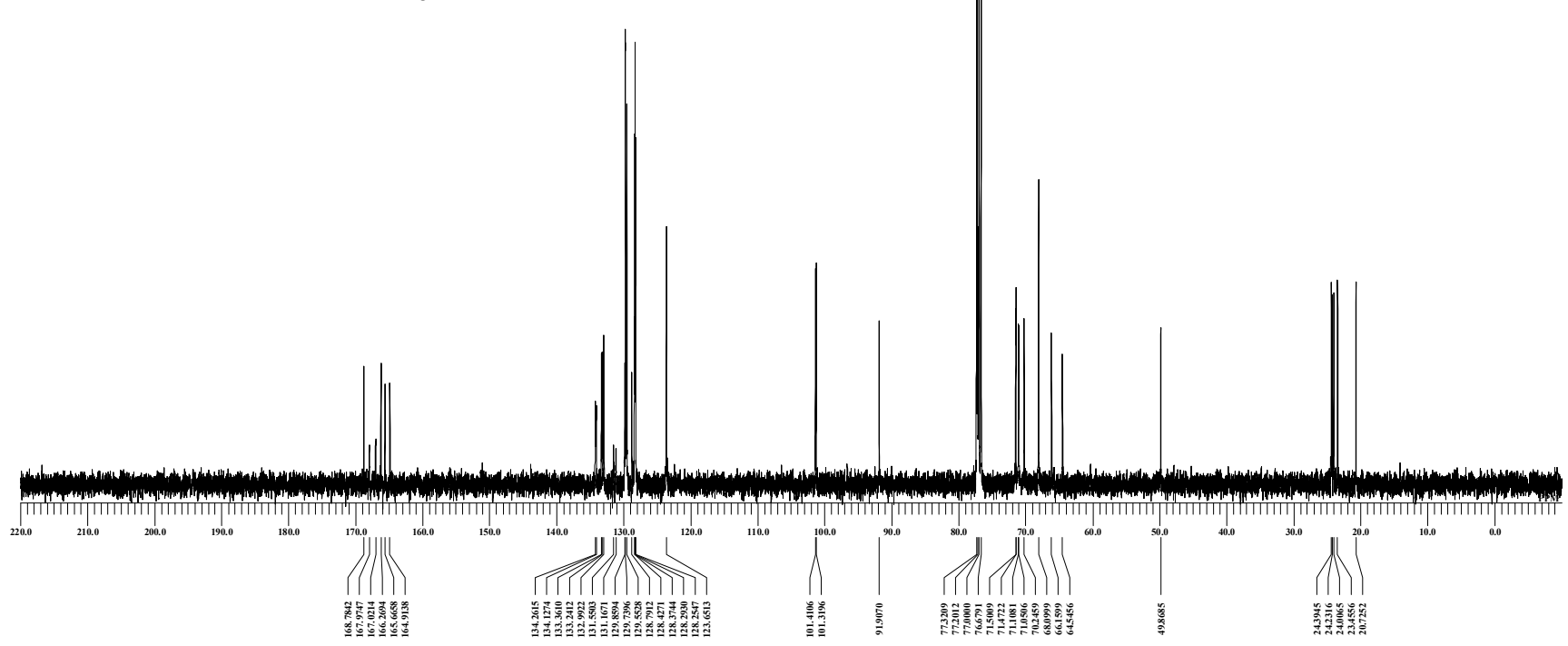




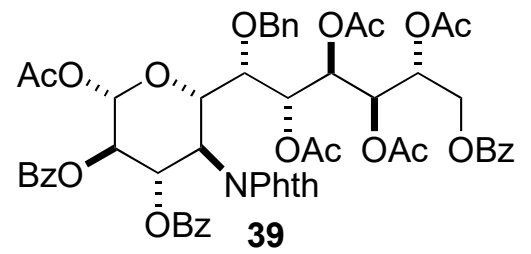

${ }^{1} \mathrm{H}$ NMR $\left(400 \mathrm{MHz}, \mathrm{CDCl}_{3}\right)$

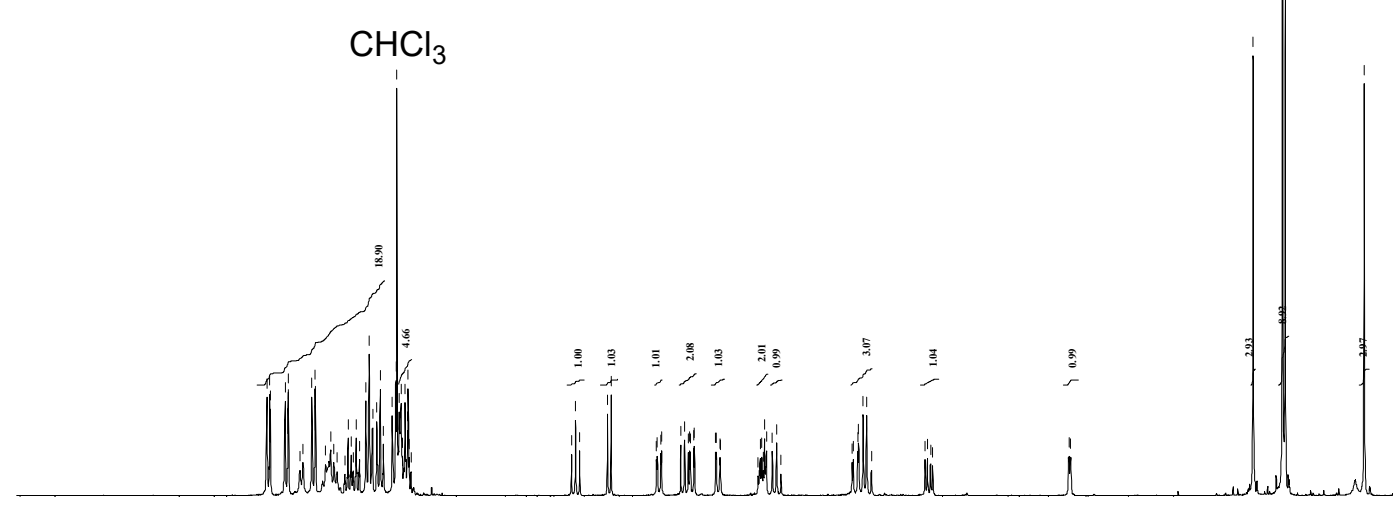

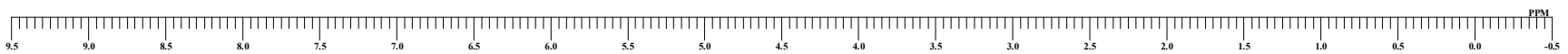

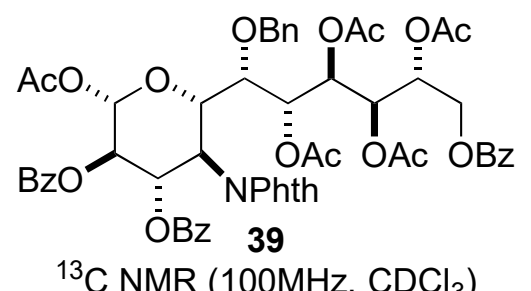
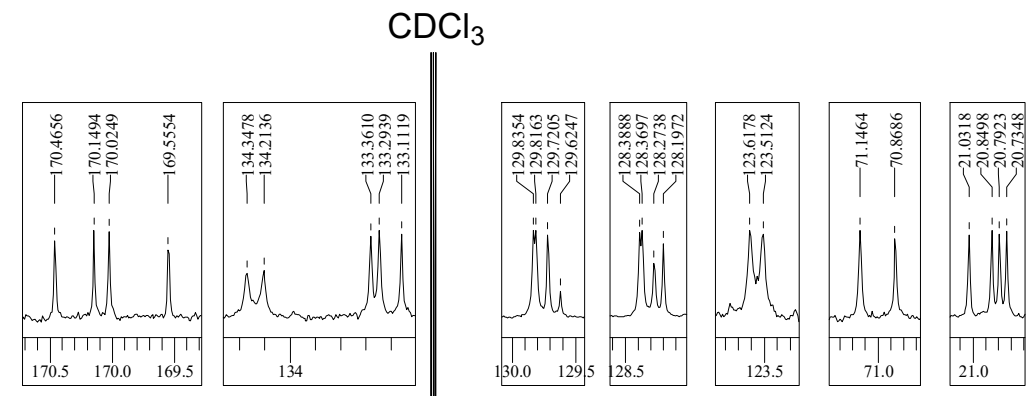

${ }^{13} \mathrm{C} \mathrm{NMR}\left(100 \mathrm{MHz}, \mathrm{CDCl}_{3}\right)$

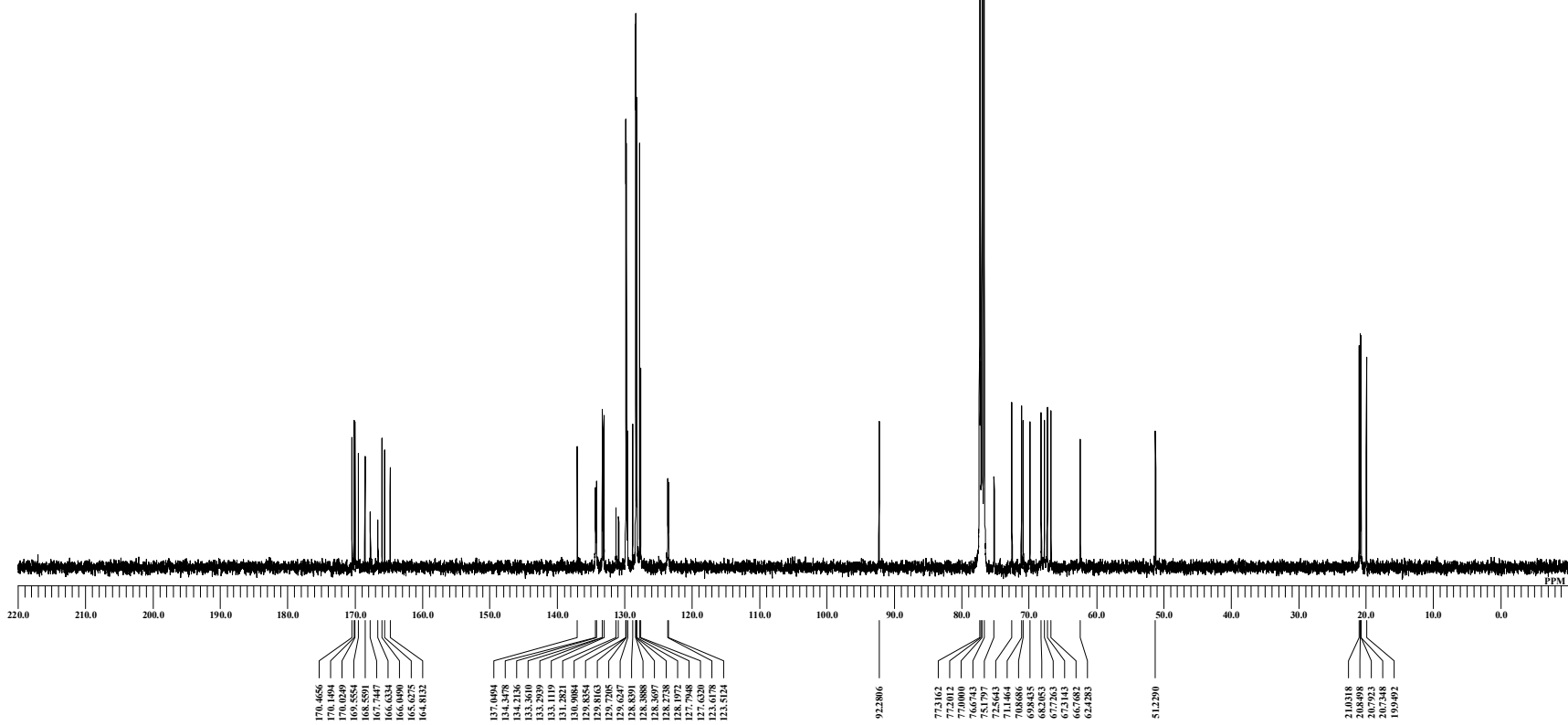


$\mathrm{BzHN}$

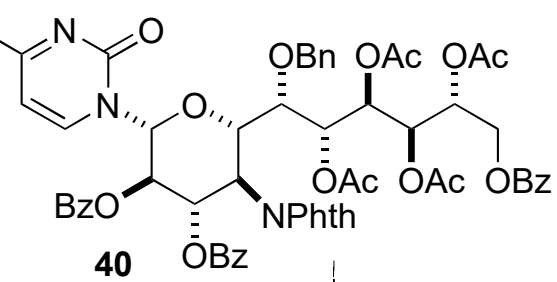

${ }^{1} \mathrm{H}$ NMR $\left(400 \mathrm{MHz}, \mathrm{CDCl}_{3}\right.$ )
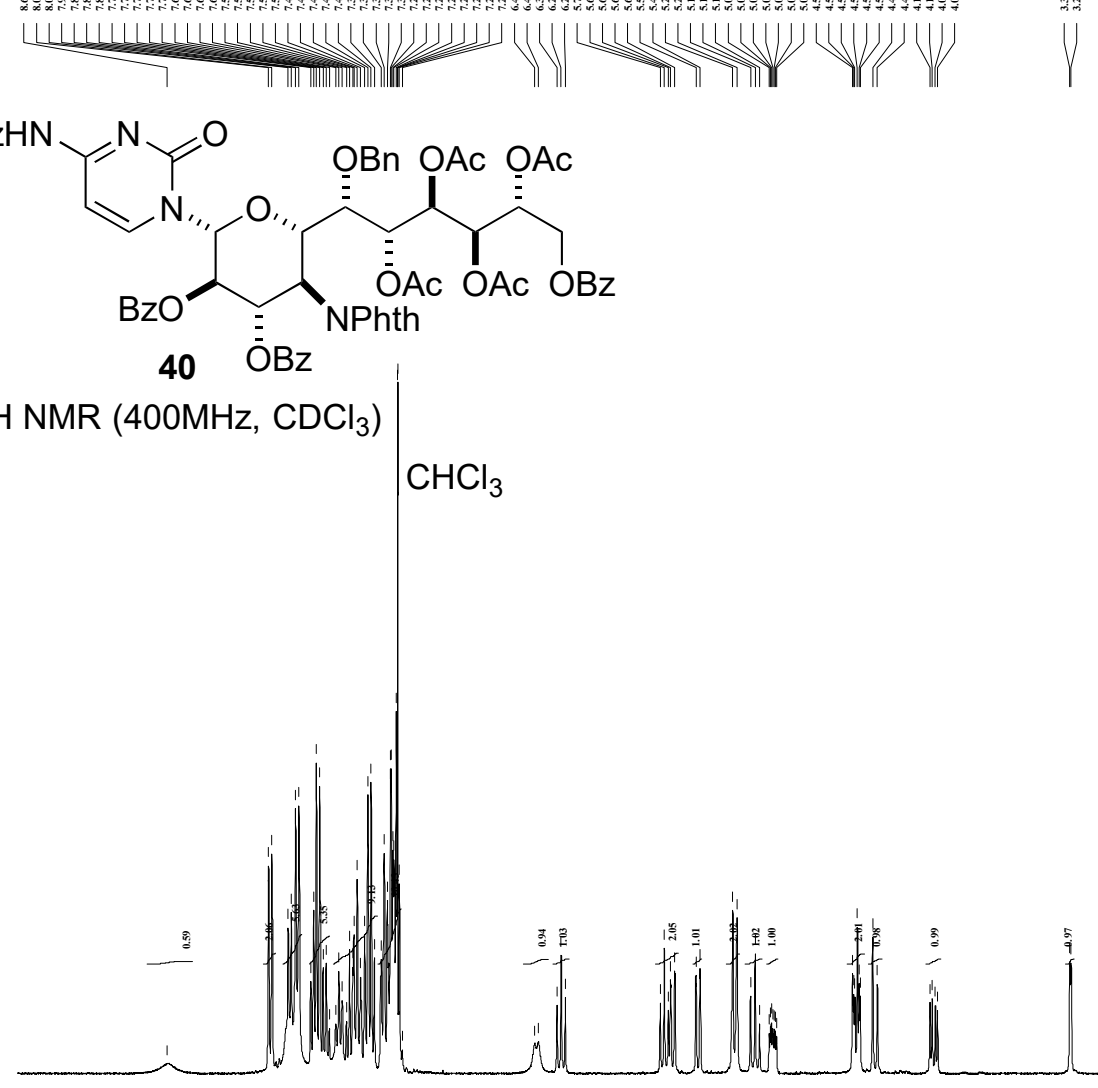

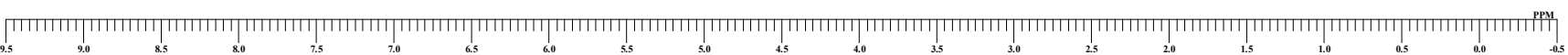

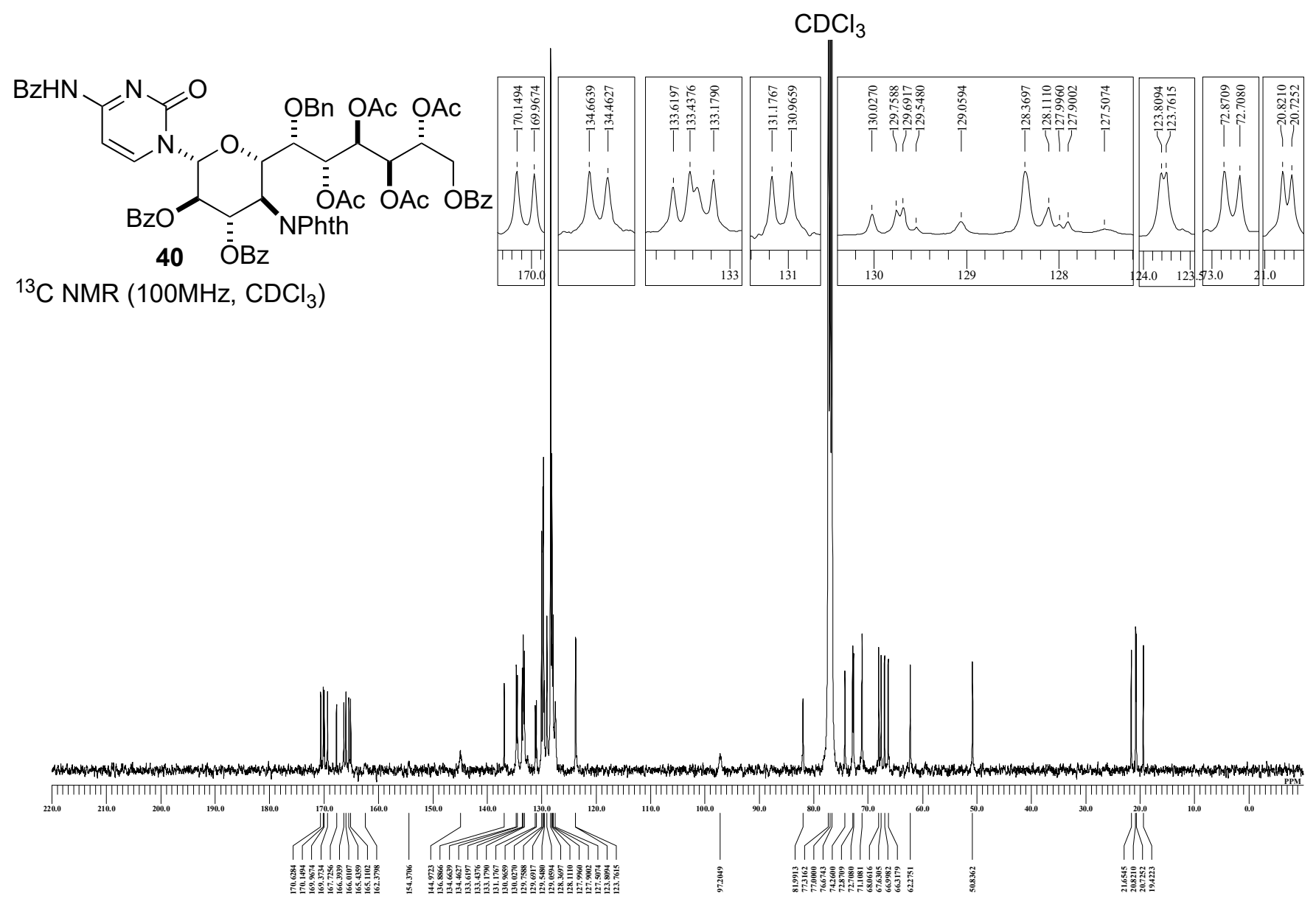



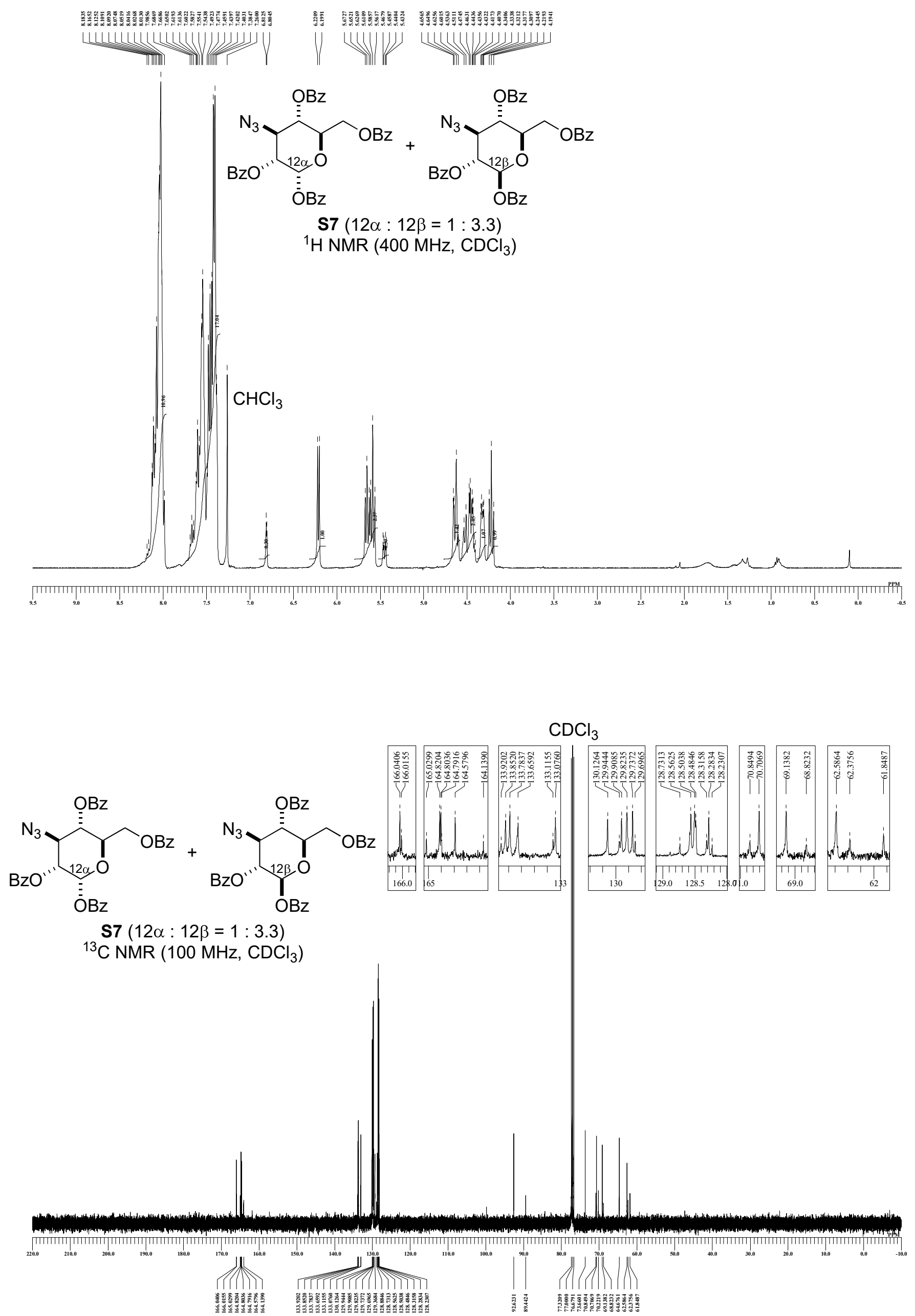

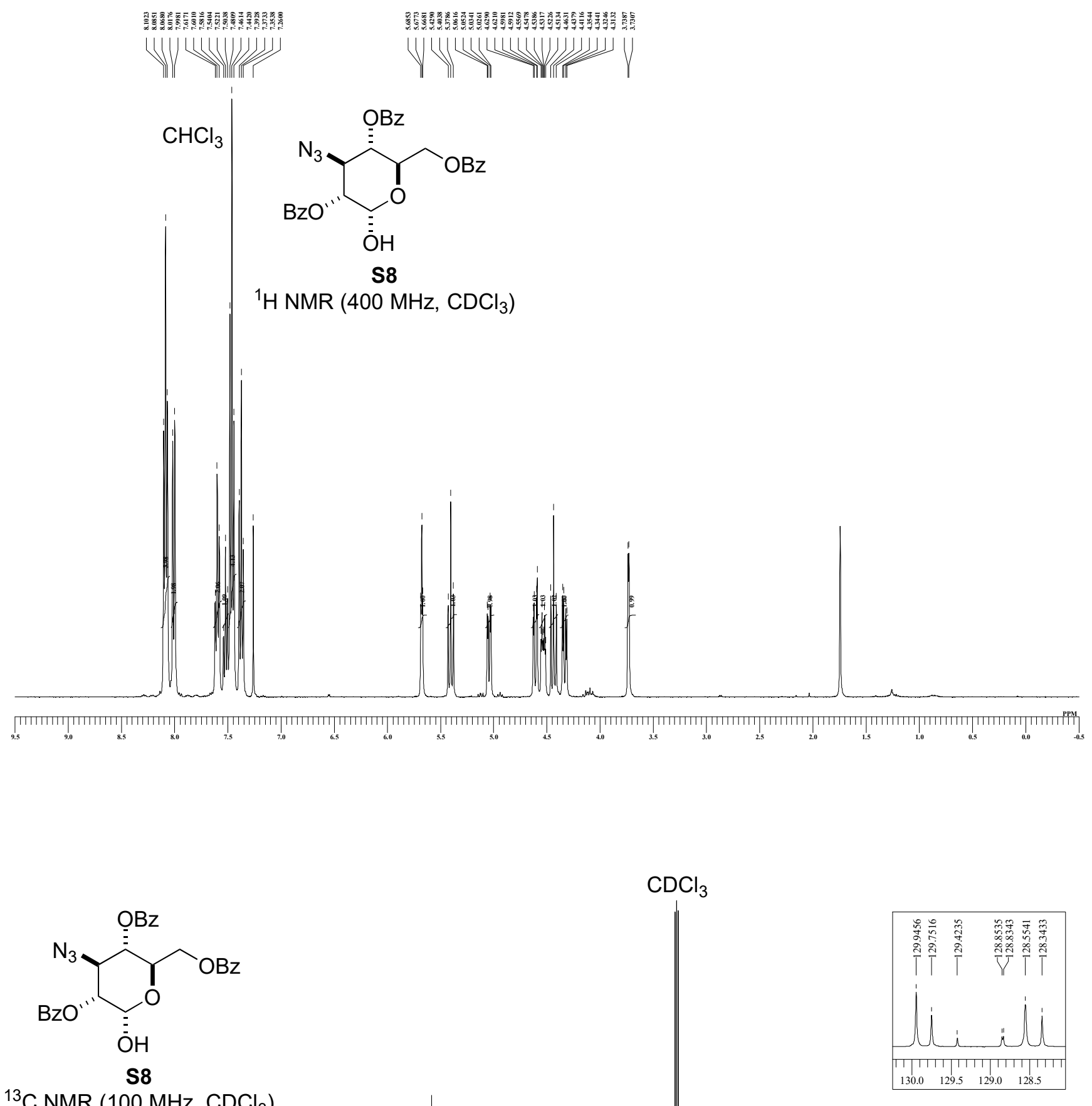

${ }^{13} \mathrm{C}$ NMR $\left(100 \mathrm{MHz}, \mathrm{CDCl}_{3}\right)$

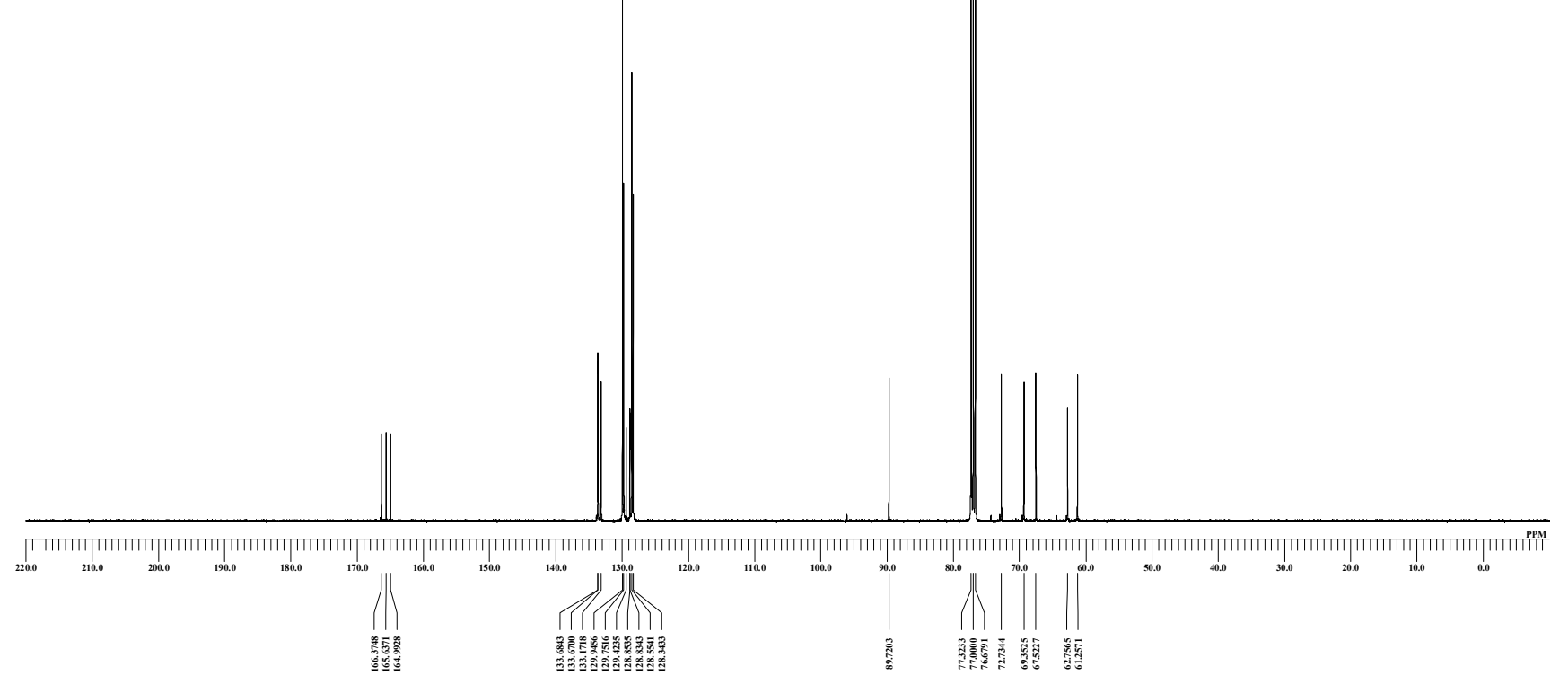




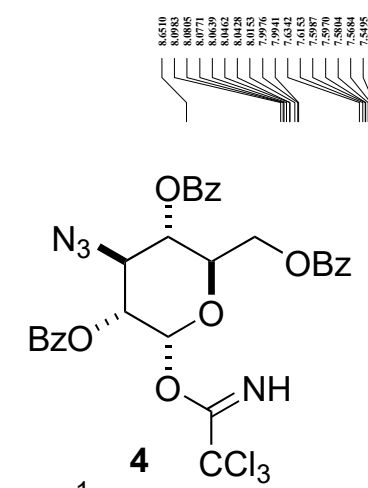

${ }^{1} \mathrm{H}$ NMR

$\left(400 \mathrm{MHz} \mathrm{CDCl}_{3}\right)$

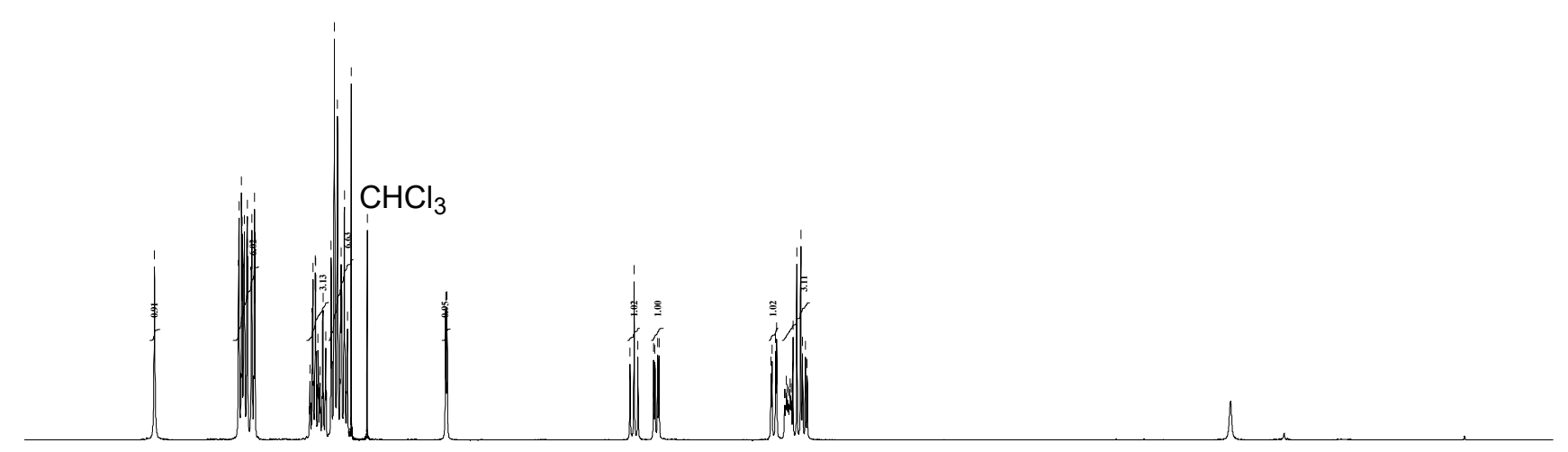

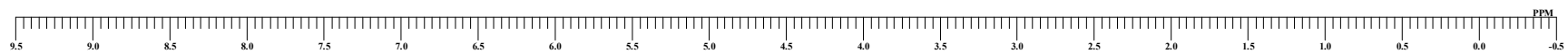

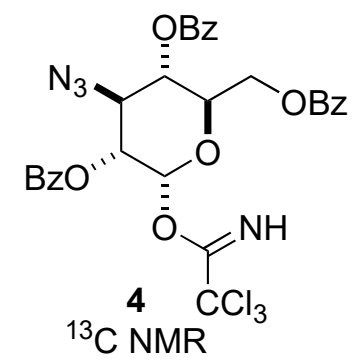

$\mathrm{CDCl}_{3}$

$\left(100 \mathrm{MHz}, \mathrm{CDCl}_{3}\right.$ )

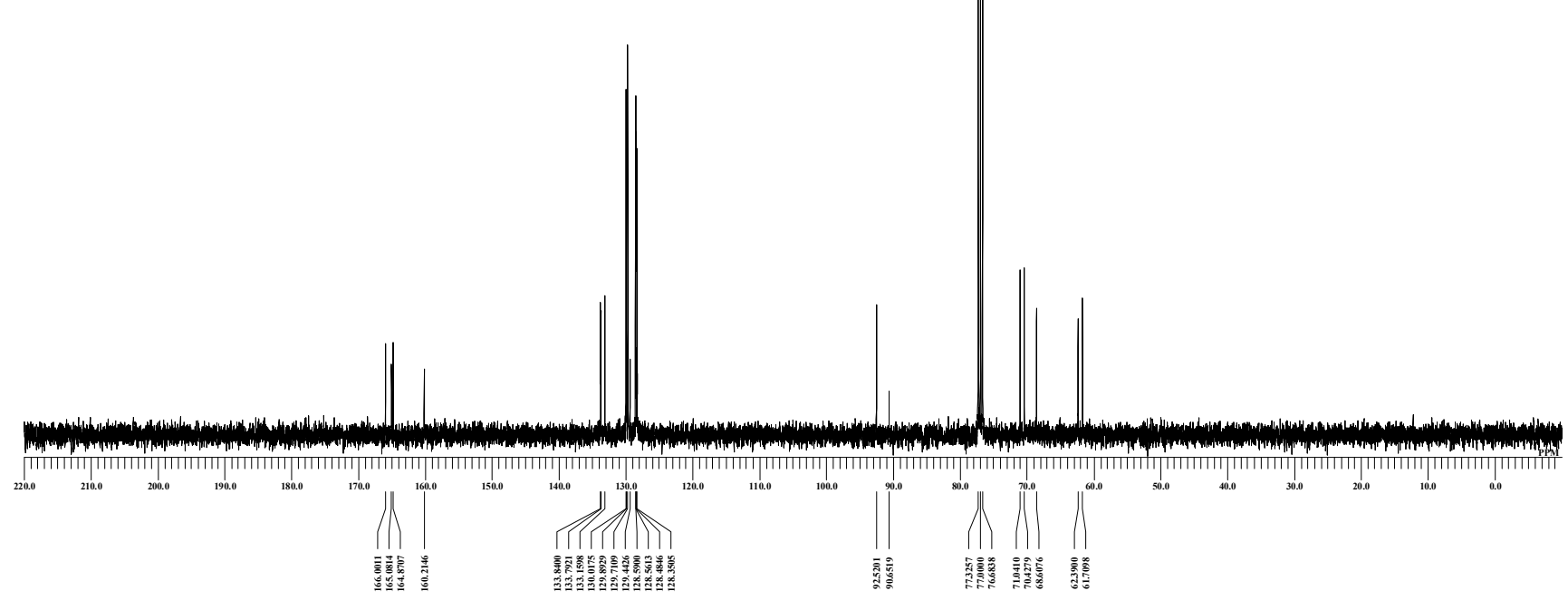




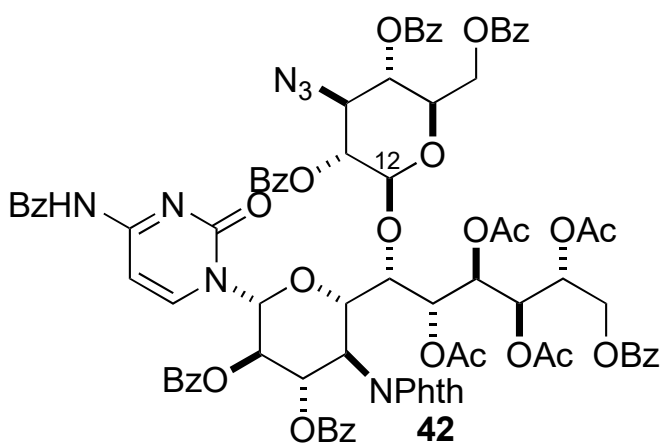

${ }^{1} \mathrm{H}$ NMR $\left(500 \mathrm{MHz}, \mathrm{CO}\left(\mathrm{CD}_{3}\right)_{2}\right)$
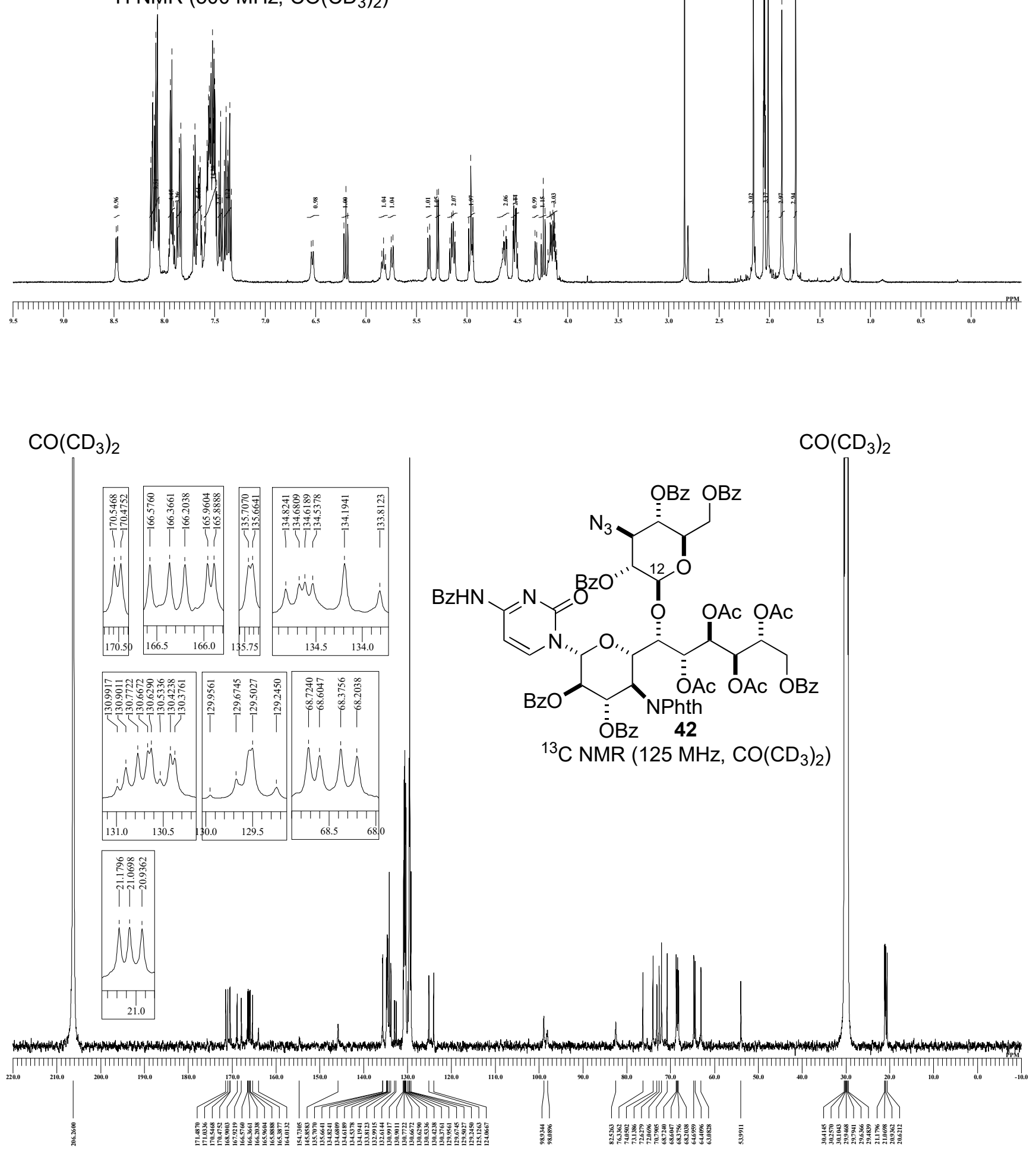


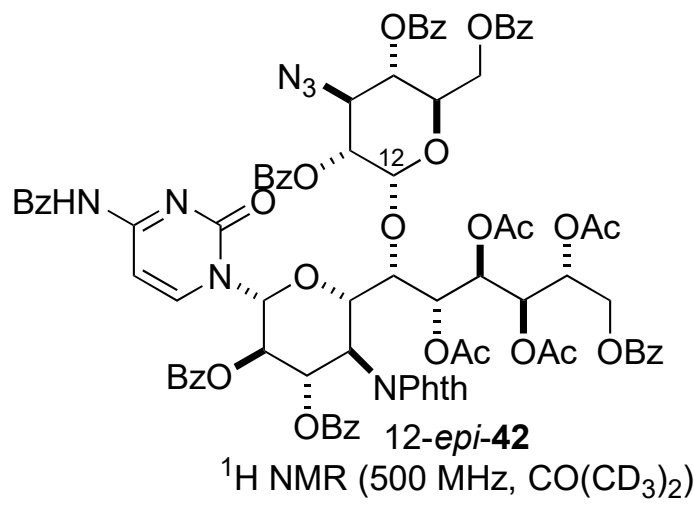

${ }^{1} \mathrm{H}$ NMR $\left(500 \mathrm{MHz}, \mathrm{CO}\left(\mathrm{CD}_{3}\right)_{2}\right)$

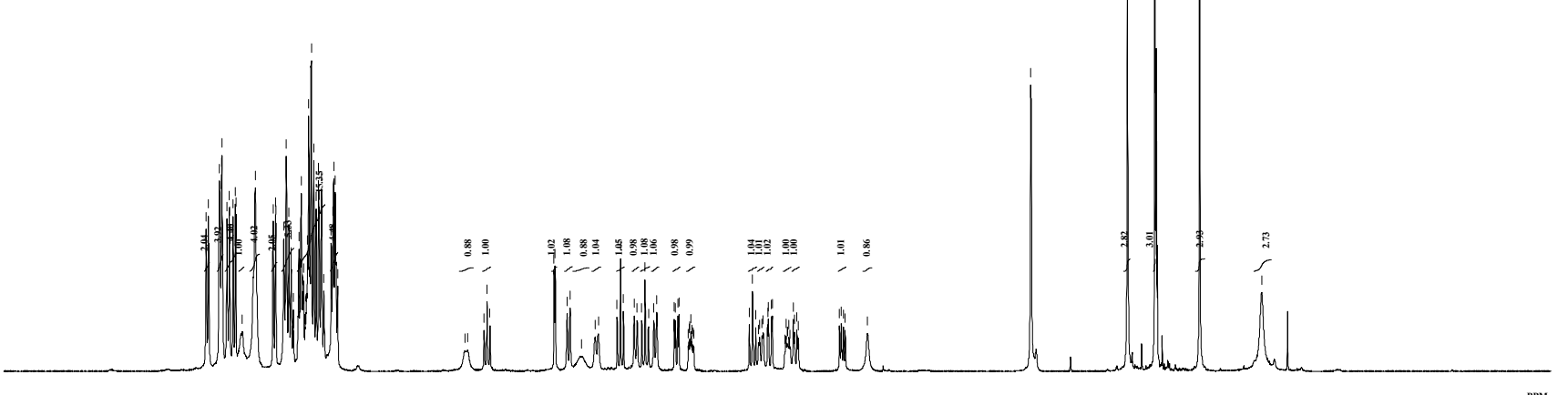

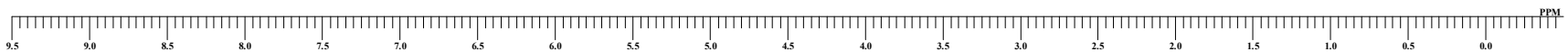

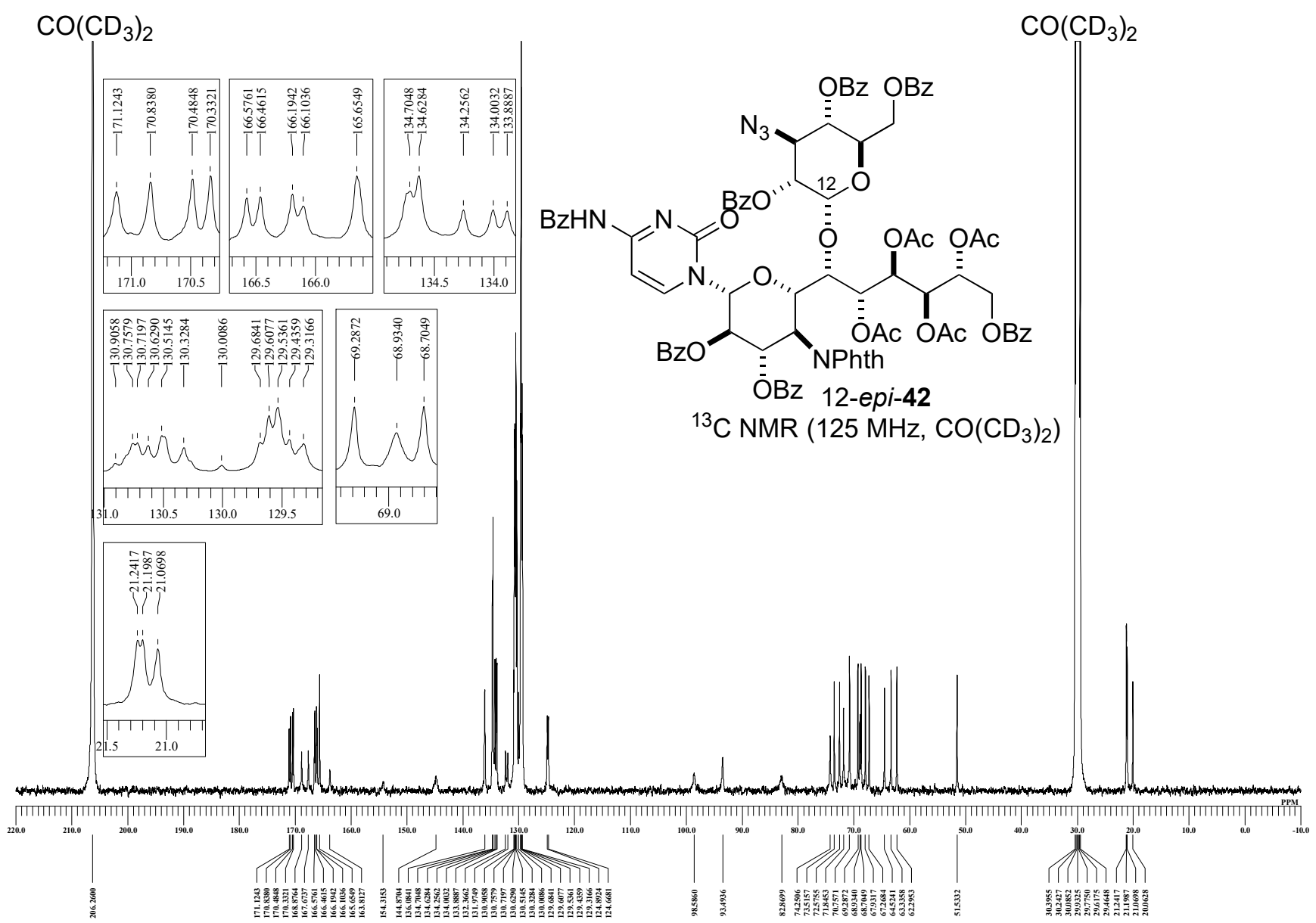



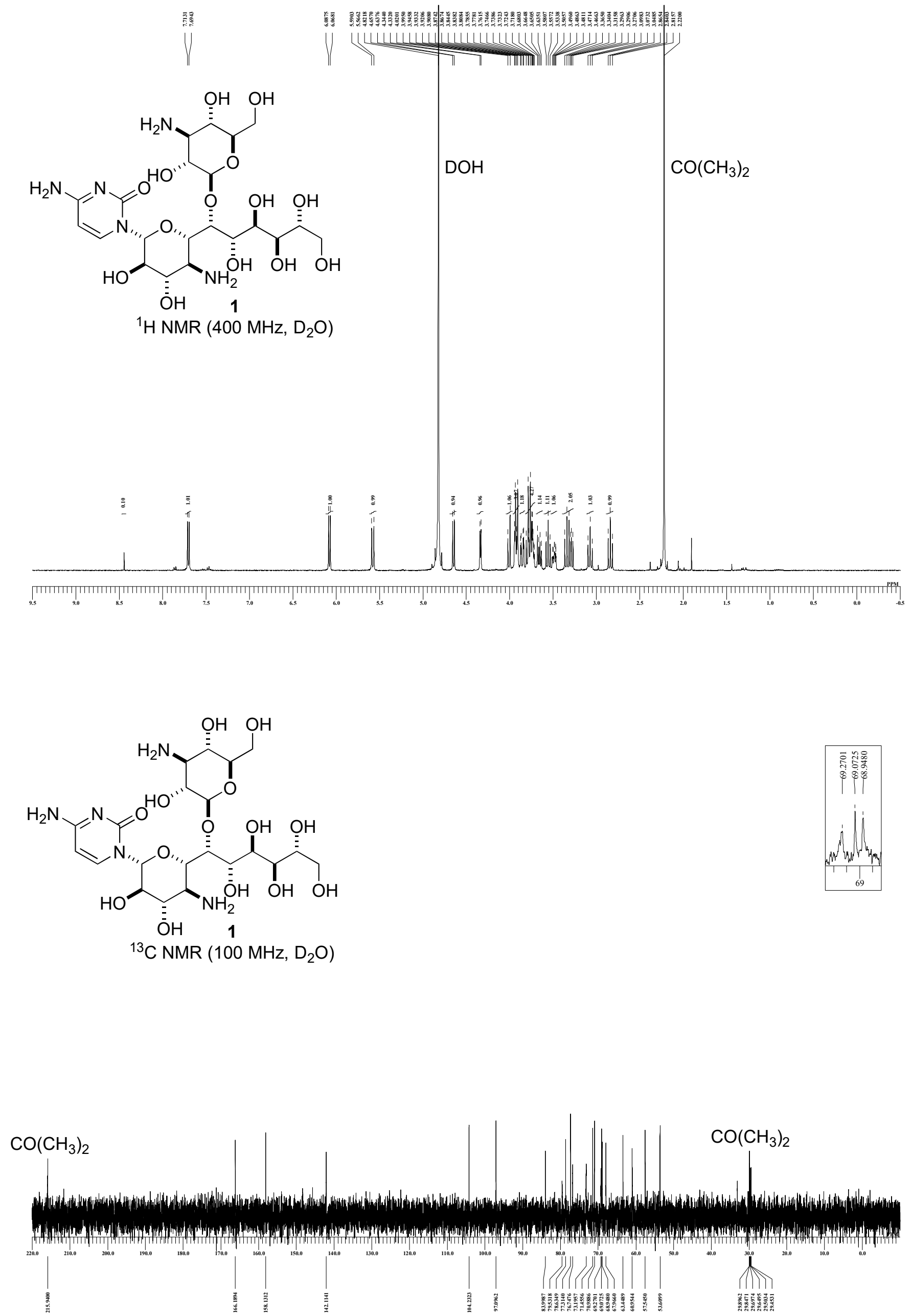


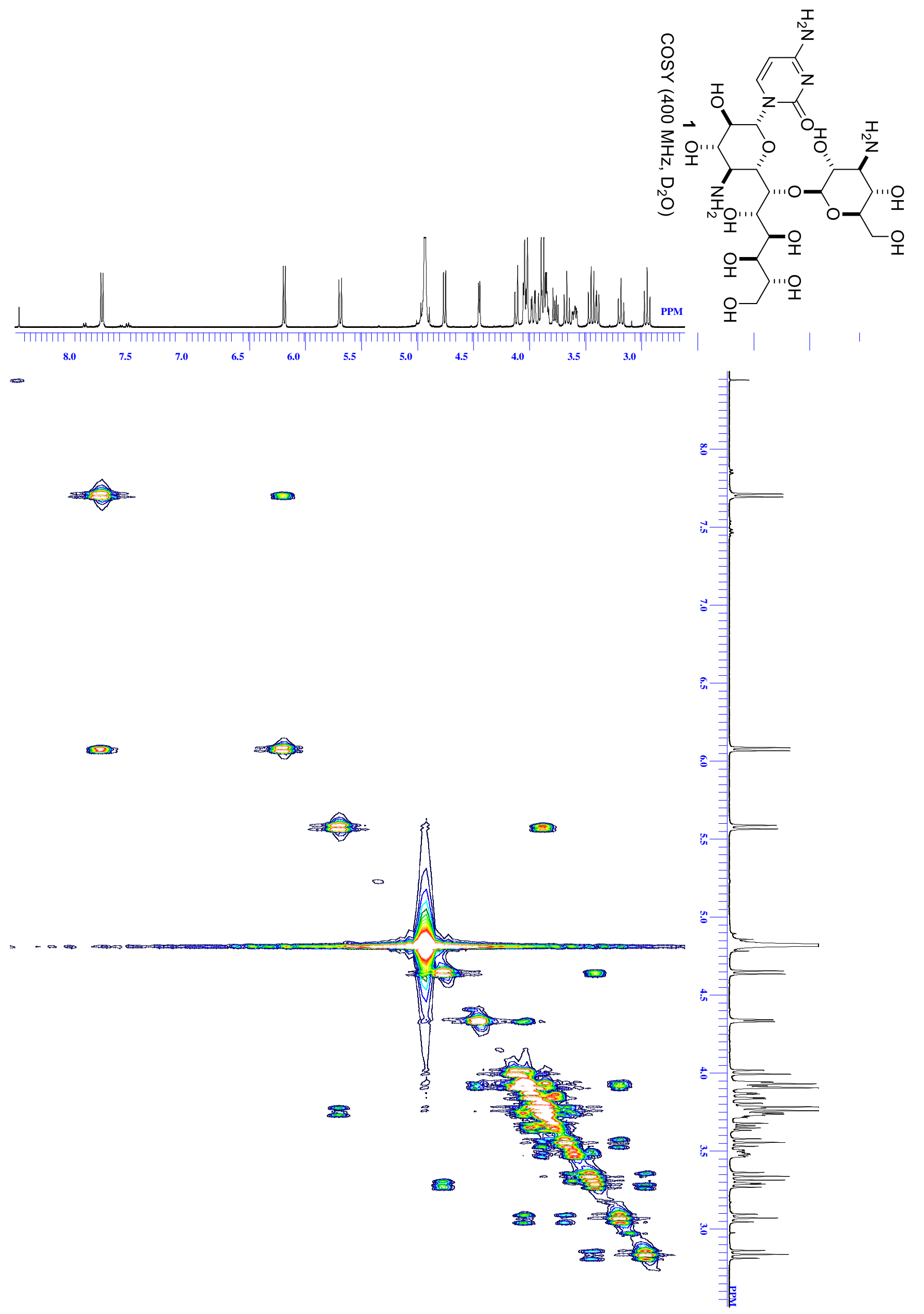




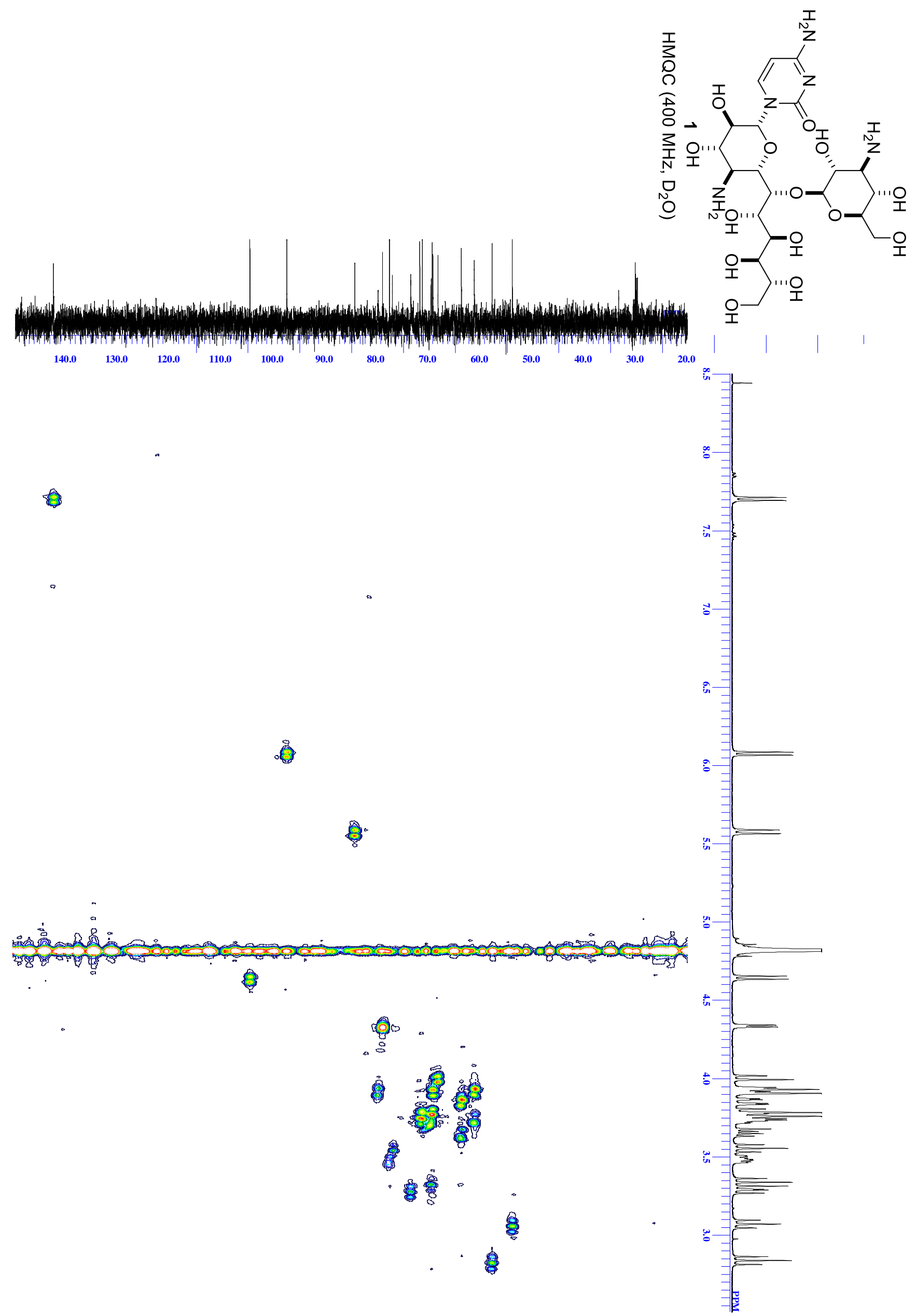




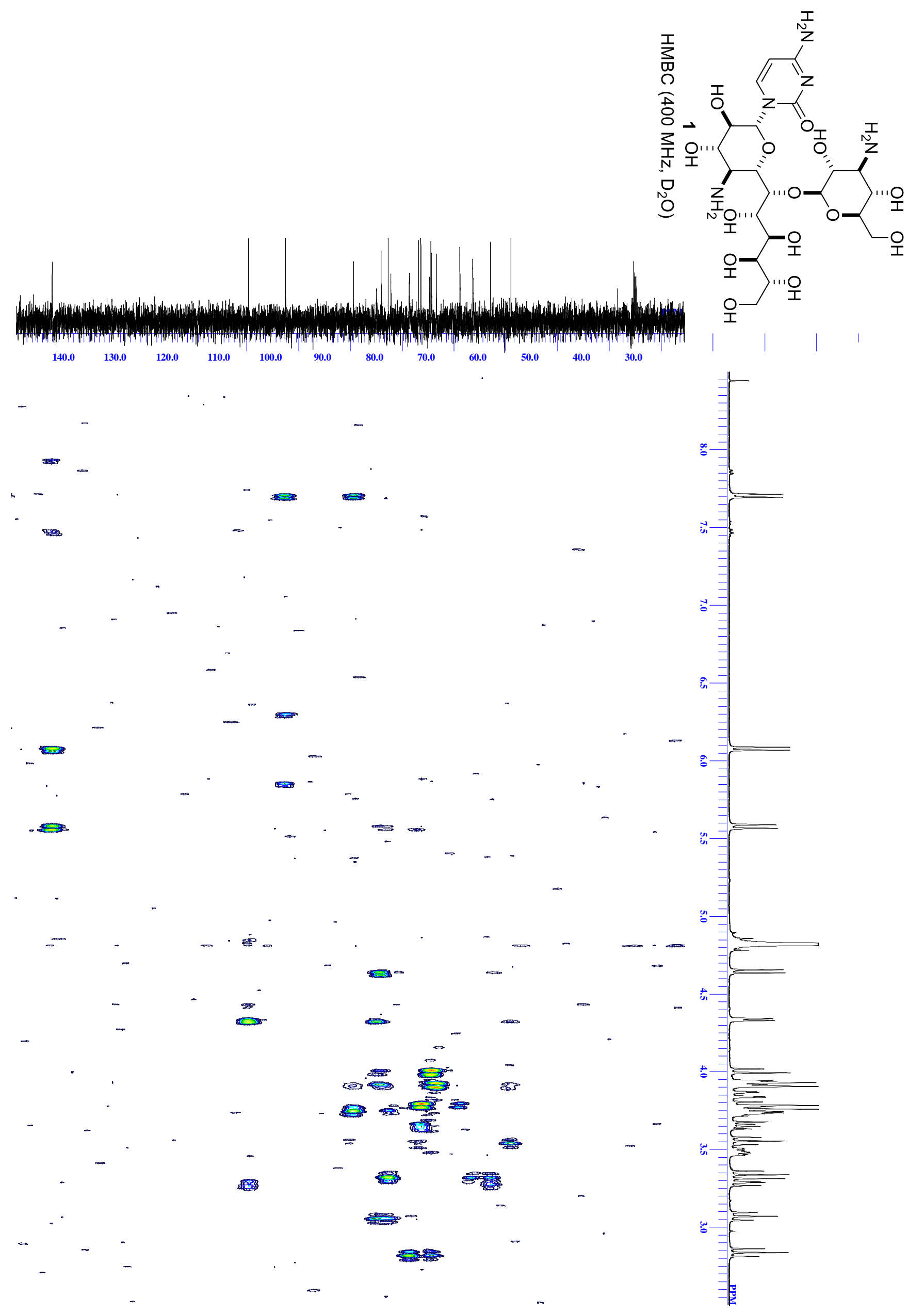




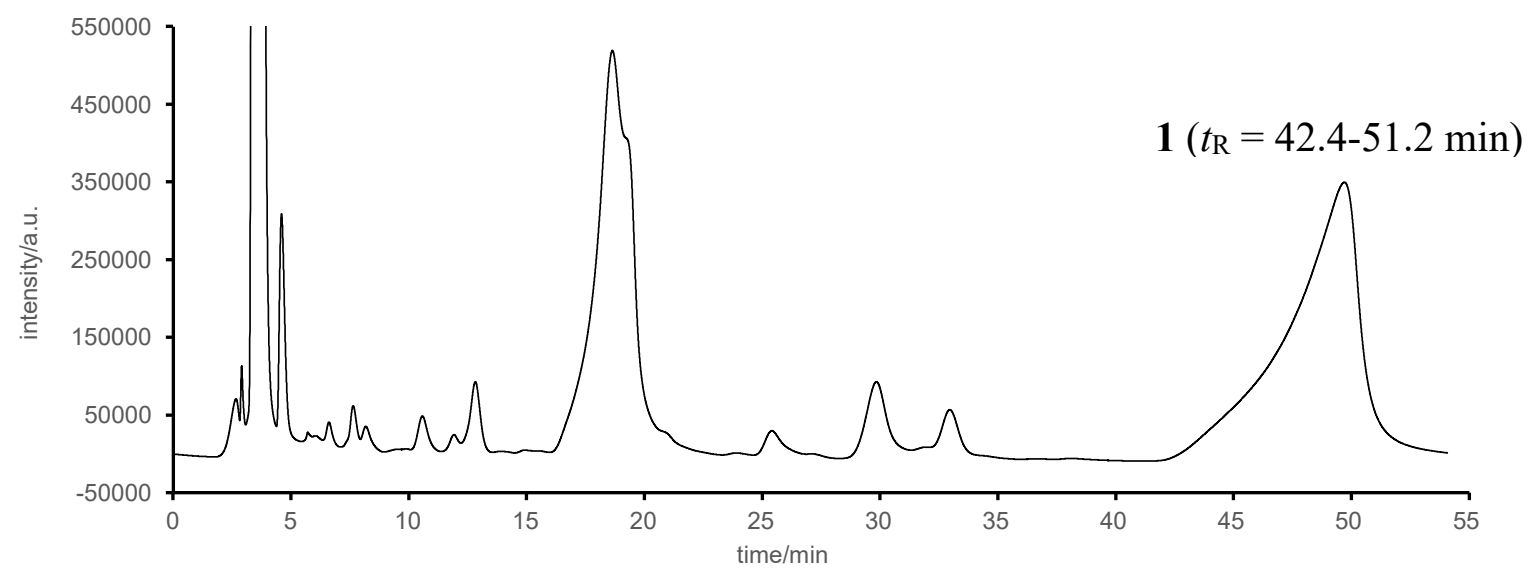

Figure S5. HPLC chart for purification of 1. Column: Shodex Asahipak NH2P-50 10E $10 \times$ $250 \mathrm{~mm}$, eluent: $\mathrm{MeCN} / \mathrm{H}_{2} \mathrm{O}=75 / 25$, flow rate: $3.0 \mathrm{~mL} / \mathrm{min}$, detection: photodiode array detector 199-599nm (UV chromatogram: $254 \mathrm{~nm}$ ), temperature: $25^{\circ} \mathrm{C}$ 